\title{
WestVirginiaUniversity
}

THE RESEARCH REPOSITORY @ WVU

Graduate Theses, Dissertations, and Problem Reports

2015

\section{The Nature of Magnetic Reconnection at the Dayside \\ Magnetopause}

\author{
Colin Michael Komar
}

Follow this and additional works at: https://researchrepository.wvu.edu/etd

\section{Recommended Citation}

Komar, Colin Michael, "The Nature of Magnetic Reconnection at the Dayside Magnetopause" (2015). Graduate Theses, Dissertations, and Problem Reports. 6003.

https://researchrepository.wvu.edu/etd/6003

This Dissertation is protected by copyright and/or related rights. It has been brought to you by the The Research Repository @ WVU with permission from the rights-holder(s). You are free to use this Dissertation in any way that is permitted by the copyright and related rights legislation that applies to your use. For other uses you must obtain permission from the rights-holder(s) directly, unless additional rights are indicated by a Creative Commons license in the record and/ or on the work itself. This Dissertation has been accepted for inclusion in WVU Graduate Theses, Dissertations, and Problem Reports collection by an authorized administrator of The Research Repository @ WVU.

For more information, please contact researchrepository@mail.wvu.edu. 


\title{
The Nature of Magnetic Reconnection at the Dayside Magnetopause
}

\author{
Colin Michael Komar \\ Dissertation submitted \\ to the Eberly College of Arts and Sciences \\ at West Virginia University \\ in partial fulfillment of the requirements for the degree of \\ Doctor of Philosophy in \\ Physics
}

\author{
Paul A. Cassak, Ph. D., Chair \\ Daniel Pisano, Ph. D. \\ Earl E. Scime, Ph. D. \\ Stephen Valentine, Ph. D. \\ Dimitris Vassiliadis, Ph. D. \\ Department of Physics and Astronomy
}

Morgantown, West Virginia

2015

Keywords: magnetic reconnection, magnetosphere, separators

(C) Copyright 2015 Colin Michael Komar 


\title{
ABSTRACT \\ The Nature of Magnetic Reconnection at the \\ Dayside Magnetopause
}

\author{
Colin Michael Komar
}

Magnetic reconnection is a phenomenon that occurs in hot ionized gases, or plasmas, whereby oppositely directed magnetic field components break and cross-connect, converting energy stored in the magnetic fields to plasma motion and heat. Reconnection occurs between Earth's magnetic field and the interplanetary magnetic field (IMF) carried by the solar wind, plasma emanating from the sun. Reconnection occurs at the sunward boundary of Earth's magnetosphere, the region of space that is dominated by Earth's magnetic field. However, predicting the properties of reconnection at this boundary, including basic considerations such as where reconnection occurs and how efficiently, remain an unsolved problem.

In this dissertation, we present an efficient and highly accurate method for identifying possible sites of reconnection by locating magnetic separators, magnetic field lines that separate regions of different magnetic topologies at the dayside magnetopause. The technique is verified using exact solutions for separators in an analytic magnetic field of a superposed dipolar and uniform magnetic field. Magnetic separators are found in distinct global resistive magnetohydrodynamic (MHD) simulations performed using the three-dimensional Block Adaptive Tree Solar wind Roe-type Upwind Scheme (BATS-R-US) code with a uniform resistivity with IMF orientations ranging from parallel to anti-parallel to Earth's magnetic field.

With the magnetic separators, we can make careful tests of recent models of the location of reconnection at the dayside magnetopause for arbitrary solar wind conditions. As many of these models are independent of the reconnection dissipation mechanism, each can be tested in our resistive MHD simulations. We employ image processing techniques to unambiguously determine each model's prediction in order to compare the determined separators with the models. We find that none of the models are perfect, but the maximum magnetic shear model does the best at finding the polar cusp reconnection sites for northward IMF.

We also present an initial analysis of reconnection local to the magnetic separator. This is achieved by measuring reconnection parameters in planes perpendicular to the magnetic separator and comparing the results to local models of reconnection. The development of this capability has profound implications in understanding how the reconnection physics local to the magnetic separator can lead to global magnetospheric dynamics at Earth, a longstanding problem in understanding solar wind-magnetospheric coupling. 
"I learned this, at least, by my experiment; that if one advances confidently in the direction of his dreams, and endeavors to live the life which he has imagined, he will meet with a success unexpected in common hours."

-Henry David Thoreau, Walden

This work is dedicated to:

My parents, Paul and Rosemary, who sacrificed so much of themselves to ensure their children could reach for the stars seen above the Central Illinois cornfields.

My wife, Kathryn, for putting up with the many late nights (and too many undone dishes). You continue to provide me with love, happiness, laughter, and support. You are my best friend and constant companion. (And yes, I will buy you your puppy!) 


\section{Acknowledgements}

First and foremost, I extend my most heartfelt thanks to my Ph. D. advisor, Paul Cassak. In many ways, the title "academic father" befits you since you have seen me at my best and worst throughout my graduate school career, and yet you still provided me the support necessary for my success. I am truly honored to have you as a mentor, colleague, and friend.

Additional thanks go to the members serving on my Ph. D. committee: Drs. D. J. Pisano, Earl Scime, Stephen Valentine, and Dimitris Vassiliadis. Thank you for taking the time out of your busy schedules to read through this dissertation. I also thank you for your roles as educators by facilitating my growth in knowledge and training required for a scientific career.

I also would like to extend my gratitude to my research collaborators, Drs. John Dorelli, Alex Glocer, and Masha Kuznetsova. John, Alex and Masha were instrumental in ensuring the simulation results and methods for determining separators detailed in this dissertation were valid. I would like to additionally thank Joe Borovsky, Stephen Fuselier, Gabor Tóth, Ray Lopez, Yi-Hsin Liu, Tom Moore, Goetz Paschmann, Steve Petrinec, David Sibeck, Karlheinz Trattner, and Brian Walsh for their insight and interesting discussions.

Thanks go to the staff of NASA's Community Coordinated Modeling Center (CCMC) at Goddard Space Flight Center (http://ccmc.gsfc.nasa.gov) for their software and simulation support: Anna Chulaki, Marlo Maddox, Anne Michelle Mendoza, and Lutz Rastaetter. The CCMC is a multi-agency partnership between NASA, AFMC, AFOSR, AFRL, AFWA, NOAA, NSF and ONR. Simulations were performed at the CCMC through their public Runs on Request system. The BATS-R-US Model was developed by the Center for Space Environment Modeling at the University of Michigan. A large portion of the analysis presented 
here was made possible via the Kameleon and Space Weather Explorer software packages provided by the CCMC. Software Developers: Marlo M. Maddox, David H. Berrios, and Lutz Rastaetter.

I thank my colleagues in the Cassak research group over these past several years who have informed me of the physics that excites them and greatly helped me progress in my own. Specifically, I would like to thank Rob Baylor, Matt Beidler, Chris Doss, Ray Fermo, and Luke Shepherd. May you all be successful in your future endeavors!

Many thanks go to the faculty and staff of the West Virginia University Department of Physics and Astronomy for the gift of knowledge and constant support of their students. Additional thanks to Sherry Puskar, Viola Bryant, and Vanessa Moyers for your help in completing the mountains of paperwork relating to travel, reimbursements, and graduation.

Thank you to the many friends that I have made here at WVU these past seven years: Jerry Carr, Jr., Justin Elfritz, Drew Elliott, Justin Ellis, Matt Galante, Dustin McCarren, Mitch Mickaliger, Katie Rabidoux, Andrew Seymour, and Spencer Wolfe. I will often think fondly of our times together and may you too be successful in all of your endeavors!

Finally, many thanks to Katie, Mom, Dad, Aaron, Genny, Scott Komar, Sherry Phillips, and Mommy Meyers. You all have supported me through the arduous task of completing my Ph. D. You also understood the sacrifices I was making for my future career. I thank you for your love and support over these past several years and I hope for its continuation into the future. I love you all!

Travel and funding support from the NASA West Virginia Space Grant Consortium, National Science Foundation, CCMC, and Geospace Environment Modeling Workshop is gratefully acknowledged. 


\section{Table of Contents}

List of Figures . . . . . . . . . . . . . . . . . . . . . xi

List of Tables . . . . . . . . . . . . . . . . . . . . . $\mathrm{xv}$

1 Introduction $\quad 1$

1.1 Space Physics Primer . . . . . . . . . . . . . . . . . . 3

1.2 Magnetospheric Reconnection ... . . . . . . . . . . . . . 10

1.3 Reconnection's Importance for Space Weather . . . . . . . . . . . . . . . 15

1.3.1 Reconnection's Role in Plasmaspheric Plasma Transport . . . . . . . 16

1.3.2 Outer Radiation Belt Losses Resulting from Dayside Reconnection . . 17

1.3.3 Ring Current Energization from Magnetotail Reconnection . . . . . . 19

1.4 The Local Physics of Magnetic Reconnection . . . . . . . . . . . . . . . . . 22

1.4.1 Symmetric Reconnection . . . . . . . . . . . . . . 22

1.4.2 Asymmetric Reconnection . . . . . . . . . . . . . . . . 26

1.4.3 The Effects of Sheared Plasma Flows on Magnetic Reconnection . . . 29

1.5 The Global Picture of Magnetospheric Reconnection . . . . . . . . . . . . . . 32

1.5.1 Magnetic Separators . . . . . . . . . . . . . . 33

1.5.2 Observations of Separators at the Dayside Magnetopause . . . . . . . 37

1.5.3 Observations of Separators in the Magnetotail . . . . . . . . . . . 37 
1.6 Dayside Reconnection as Either a Global or Local Process . . . . . . . . . . 38

1.7 Statement of the Problem . . . . . . . . . . . . . . . . . . 41

1.8 Summary of Results . . . . . . . . . . . . . . . . . . . . . 42

2 Magnetospheric Simulation Study $\quad 45$

2.1 The Governing Equations of Resistive Magnetohydrodynamics . . . . . . . . 45

2.2 The Code and its Initialization _ . . . . . . . . . . . . . . . . 47

2.2.1 Numerical Test 1: Polar Cap Saturation . . . . . . . . . . . . . 50

2.2.2 Numerical Test 2: Numerical vs. Explicit Dissipation . . . . . . . . . 51

3 Tracing Magnetic Separators $\quad 55$

3.1 A Technique for Finding Separators . . . . . . . . . . . . . . 56

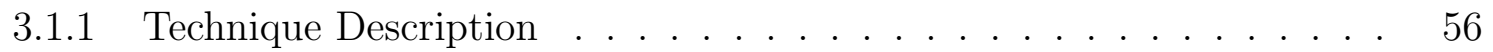

3.1.2 Verification with Vacuum Superposition . . . . . . . . . . . 61

3.2 Results . . . . . . . . . . . . . . . . . . . . . 63

3.2.1 The Magnetic Nulls . . . . . . . . . . . . . . . . . . . . . 64

3.2.2 The Magnetic Separators . . . . . . . . . . . . . . . . 67

3.3 Summary . . . . . . . . . . . . . . . . . . . . . 75

4 Comparative Analysis of Dayside Magnetic Reconnection Models $\quad 77$

4.1 Reconnection Location Models . . . . . . . . . . . . . . . . . . . . . . 80

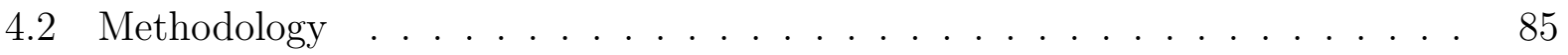

4.2.1 Identification of the Magnetopause and Plasma Parameter Sampling • 85

4.2.2 Finding Model Predictions Using Image Processing Techniques . . . . 89

4.2.3 Determining Magnetic Separators . . . . . . . . . . . . . . . . 91

4.3 Results . . . . . . . . . . . . . . . . . . . . . . 92 
4.3.1 Clock Angle Dependence . . . . . . . . . . . . . . . . . . . . . . . 94

4.3.2 IMF Strength Dependence . . . . . . . . . . . . . . . . . 98

4.3.3 Dipole Tilt Dependence . . . . . . . . . . . . . . . 100

4.3.4 Effect of a Flow Shear . . . . . . . . . . . . . . . . . . 103

4.4 Summary . . . . . . . . . . . . . . . . . . 106

5 Local Analysis of Reconnection at the Dayside Magnetopause 109

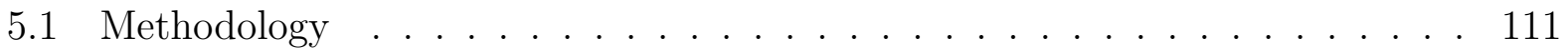

5.1.1 The Determination of Planes Normal to the Separator . . . . . . . . 111

5.1.2 Measuring Plasma Parameters in Reconnection Planes . . . . . . . . 115

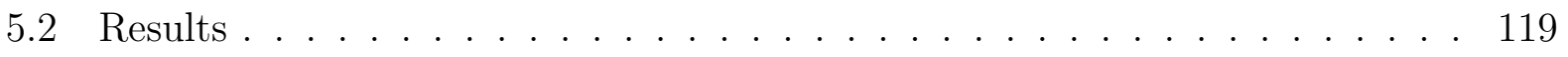

5.3 Summary . . . . . . . . . . . . . . . . . . 127

6 Conclusions $\quad 129$

6.1 Summary of Results . . . . . . . . . . . . . . . . . . . . . 129

6.1.1 Identifying Magnetic Separators in Global Magnetospheric Simulations 129

6.1.2 Comparative Analysis of Dayside Reconnection Models . . . . . . . . 130

6.1.3 Local Analysis of Reconnection at the Dayside Magnetopause . . . . 132

6.2 Limitations to the Present Work . . . . . . . . . . . . . . . 133

6.3 Future Work . . . . . . . . . . . . . . . . 136

$\begin{array}{ll}\text { A List of CCMC Simulations } & 141\end{array}$

B Overview of the BATS-R-US Simulation Code 145

C Setting up SWMF to Run on NASA's Pleiades Supercomputer $\quad 149$ 
$\begin{array}{lr}\text { D SWMF Batch Script } & 151\end{array}$

$\begin{array}{llr}\text { E SWMF PARAM.in } & 159\end{array}$

$\begin{array}{lll}\text { F } & \text { SWMF Grid File } & 165\end{array}$

$\begin{array}{ll}\text { Bibliography } & 171\end{array}$ 


\section{List of Figures}

1.1 Illustration of an Earthbound Coronal Mass Ejection . . . . . . . . . . . 2

1.2 Geocentric Solar Magnetic (GSM) Coordinate System . . . . . . . . . . . . 4

1.3 Diagram of the Solar Wind Bulk Flow and the Interplanetary Electric Field 5

1.4 Earth's Magnetosphere Under Northward and Southward IMF . . . . . . . . 7

1.5 The Polar Cap Potential at the Northern Geomagnetic Pole for Northward and Southward IMF Conditions . . . . . . . . . . . . . . 12

1.6 Diagrams of Ionospheric Convection Patterns . . . . . . . . . . . . . . . 14

1.7 Diagram of Earth's Ring Current . . . . . . . . . . . . . . . . . . . 19

1.8 Diagram of Symmetric Reconnection . . . . . . . . . . . . . . . . 23

1.9 Diagram of Asymmetric Reconnection . . . . . . . . . . . . . . . 27

1.10 Diagram of Symmetric Reconnection with a Sheared Flow . . . . . . . . . 30

1.11 Diagram of Asymmetric Reconnection with a Sheared Flow . . . . . . . . . . 31

1.12 Magnetic Topologies at the Subsolar Magnetopause and Magnetotail for Southward $\mathrm{IMF} \ldots \ldots \ldots \ldots . \ldots \ldots \ldots$

$1.13 \theta_{\mathrm{IMF}}=90^{\circ}$ Separator Surface Looking Earthward . . . . . . . . . . . 36

1.14 The Simulated Effect of Plasmaspheric Plasma on Reconnection at the Subsolar Magnetopause . . . . . . . . . . . . . . . . . . . . . 39 
2.1 Testing for Polar Cap Saturation . . . . . . . . . . . . . . 50

2.2 Comparison of Numerical and Explicit Dissipation . . . . . . . . . . . . 52

3.1 Schematic Diagram of the Separator Tracing Algorithm . . . . . . . . . . 57

3.2 Sample Topology Map for $\theta_{\mathrm{IMF}}=30^{\circ}$ Simulation . . . . . . . . . . . . . . . . 60

3.3 Verification of the Separator Tracing Algorithm . . . . . . . . . . . . . 62

3.4 Detected Separator Locations in a Simulation with $\theta_{\mathrm{IMF}}=90^{\circ} \ldots . . . . \quad 65$

3.5 Null GSM Coordinates as a Function of IMF Clock Angle . . . . . . . . . . 66

3.6 Comparison of Determined Separators with the Last Closed Field Line . . . 68

3.7 The Determined Separators as a Function of IMF Clock Angle . . . . . . . . 69

3.8 The Common Magnetic Separator Plane . . . . . . . . . . . . . . . . . 71

3.9 Separator Tilt as a Function of IMF Clock Angle . . . . . . . . . . . . 73

4.1 Schematic of the Magnetospheric Boundary Normal Coordinate system . . . 80

4.2 Example Magnetopause Determined from Current Maxima . . . . . . . . . . 87

4.3 The Determined Magnetic Shear Angle Prediction . . . . . . . . . . . . . . 91

4.4 Results for the Maximum Magnetic Shear Model . . . . . . . . . . . . . . . . 92

4.5 Results for the Asymmetric Outflow Speed Model . . . . . . . . . . . . 93

4.6 Results for the Asymmetric Sweet-Parker Reconnection Rate Model . . . . . 94

4.7 Results for the Angle of Bisection . . . . . . . . . . . . . . . . . 95

4.8 Results for the Current Density Magnitude Model . . . . . . . . . . . . 96

4.9 Results for the Uniform Guide Magnetic Field Model . . . . . . . . . . . . . 97

4.10 Results for Testing Model Dependence on IMF Strength . . . . . . . . . . . 99

4.11 Results from a Simulation with a Dipole Tilt and $\theta_{\mathrm{IMF}}=120^{\circ} \ldots$. . . . . 101

4.12 Results from a Simulation with a Dipole Tilt and $\theta_{\mathrm{IMF}}=30^{\circ} \ldots$. . . . . 102 
4.13 Testing a Sheared Flow's Effects on Asymmetric Model Predictions . . . . . 105

5.1 The Plane Normal to the Separator at the Subsolar Magnetopause in a Simulation with $\theta_{\mathrm{IMF}}=90^{\circ} \ldots \ldots \ldots \ldots \ldots$

5.2 Determination of the Upstream Plasma Parameters . . . . . . . . . . 117

5.3 Determination of the Dissipation Region Length . . . . . . . . . . . . . . 119

5.4 Reconnection Rate Comparison Along the Entire Separator for Southward IMF Orientations . . . . . . . . . . . . . . . . . . 120

5.5 Reconnection Rate Comparison for a Simulation with Dipole Tilt and Southward IMF Orientation . . . . . . . . . . . . . . . . . . . . 122

5.6 Reconnection Rate Comparison in Simulations with Lower IMF Strengths in Simulations with a Southward Orientation . . . . . . . . . . . . . . 124

5.7 Measured Dissipation Region Half-Width and Magnetic Field Ratio Along the Separator in Simulations for Lower IMF Strengths with Southward IMF

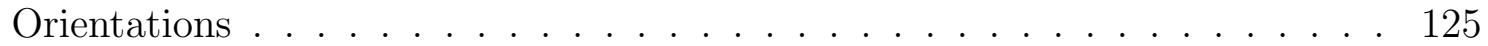

6.1 Global Separator in a Simulation with a Southward IMF Orientation . . . . 134 


\section{List of Tables}

3.1 GSM Coordinates of the Determined Magnetic Nulls in Simulations . . . . . 64

A.1 Chapter 3 Simulations . . . . . . . . . . . . . . . . . . . 142

A.2 Chapter 4 Simulations. . . . . . . . . . . . . . . . . . . . . . 142

A.3 Chapter 5 Simulations. . . . . . . . . . . . . . . . . . 143 


\section{Chapter 1}

\section{Introduction}

The region of space near Earth is very dynamic and can have a direct impact on our modern world. There are processes in near-Earth space and on Earth's surface directly driven by the interaction of Earth's magnetic field with hot ionized gas, also known as plasma, emanating from the sun. The summers of 2013 and 2014 marked the onset of and subsequent decline from solar maximum, the period of time where solar storms are quite common; solar storms include, but are not limited to, solar flares and the coronal mass ejection (CME) depicted in Fig. 1.1. These "storms from the sun" can release vast quantities of solar plasma into space; should the solar material released in these eruptions make contact with Earth's magnetic field, it can cause deleterious effects for us on Earth (National Research Council, 2002). One example is the damage done to electrical systems which can result in widespread blackouts. In March 1989, $\$ 10 \mathrm{M}$ of electrical equipment in the power grid was damaged by powerful currents resulting from interactions with a severe solar storm, which left Québec and portions of New England without power. Another threat is the increase in radiation levels which could be harmful for astronauts on space walks. It is estimated that if Apollo 17 had launched in early August 1972, astronauts would have exceeded their career limits of acceptable radiation 


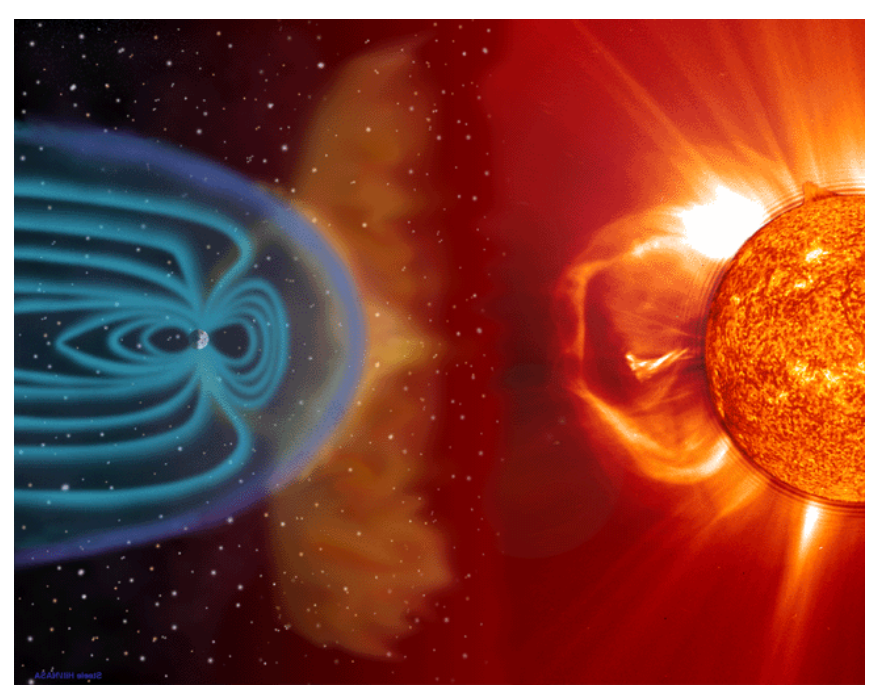

Figure 1.1: Illustration of a coronal mass ejection at the sun (right) and a few days later the interplanetary coronal mass ejection cloud encountering Earth's magnetic field depicted as light blue lines (left); depicted features are not to scale. Courtesy of SOHO/EIT/LASCO consortium. SOHO is a project of international cooperation between ESA and NASA.

exposure to skin after only eleven hours of irradiation from solar protons released in the solar flare on August 4 of that year. Another example is damage to orbiting satellites. In May 1998, solar storms disabled the Galaxy IV telecommunication satellite, disrupting $90 \%$ of pagers in the United States. It has been estimated that the cumulative societal and economic damage from an extremely severe solar storm could cost the world economy $\$ 1-2$ trillion and require an entire decade dedicated to repairing infrastructure (National Research Council, 2008).

Due to the serious effects on space- and terrestrially-based technology, there is a pressing need to understand the causal link between solar storms and the dynamics of Earth's space environment. Space weather is the field of study that attempts to predict solar eruptions and how they impact Earth's space environment. One aspect of space weather is understanding the interaction between the solar and geomagnetic fields, including the phenomenon known as magnetic reconnection. In fact, we stand at the dawn of an exciting era in space physics 
with a great opportunity to increase our understanding of magnetic reconnection throughout the near-Earth space environment. NASA launched its Magnetospheric Multi-Scale (MMS) mission on March 12, 2015; MMS is a tetrahedral constellation of satellites that will measure the structure of magnetic reconnection at the unprecedented length scale of about 6 miles some 40,000 miles from Earth!

In this dissertation, we study how and where magnetic reconnection occurs at the location where solar material impacts Earth's magnetic field. Before we present an introduction to magnetic reconnection, it is necessary to present a brief primer on the relevant physics of the near-Earth space environment.

\section{$1.1 \quad$ Space Physics Primer}

It is convenient to define a coordinate system in the reference frame where Earth is at rest. One common choice is the Geocentric Solar Magnetic (GSM) system. The GSM coordinate system is sketched in Fig. 1.2 with the sun far to the right in (a) and with (b) being the view from the sun. The dashed line in (a) is the direction of Earth's magnetic dipole. The Earth is divided into the dayside and nightside sectors as would be seen by an observer on Earth. For that same observer, Earth's eastward rotation defines the dawn and dusk sectors. The GSM system is centered at Earth, with $\hat{\mathbf{x}}$ pointing from Earth to the sun, $\hat{\mathbf{y}}$ perpendicular to Earth's magnetic dipole axis and pointing from dawn to dusk, and $\hat{\mathbf{z}}$ completes the righthanded triplet. This system fixes Earth's dipole axis to lie within the $x$ - $z$ plane, and the angle $\psi$ measures the angle the magnetic dipole axis makes with the $+z$ axis, where $\psi>0$ has the northern geomagnetic pole pointing sunward. We note that Earth's rotational axis with rotational frequency $\Omega_{E}$ is oriented at $\approx 11^{\circ}$ from the dipole axis and need not be 

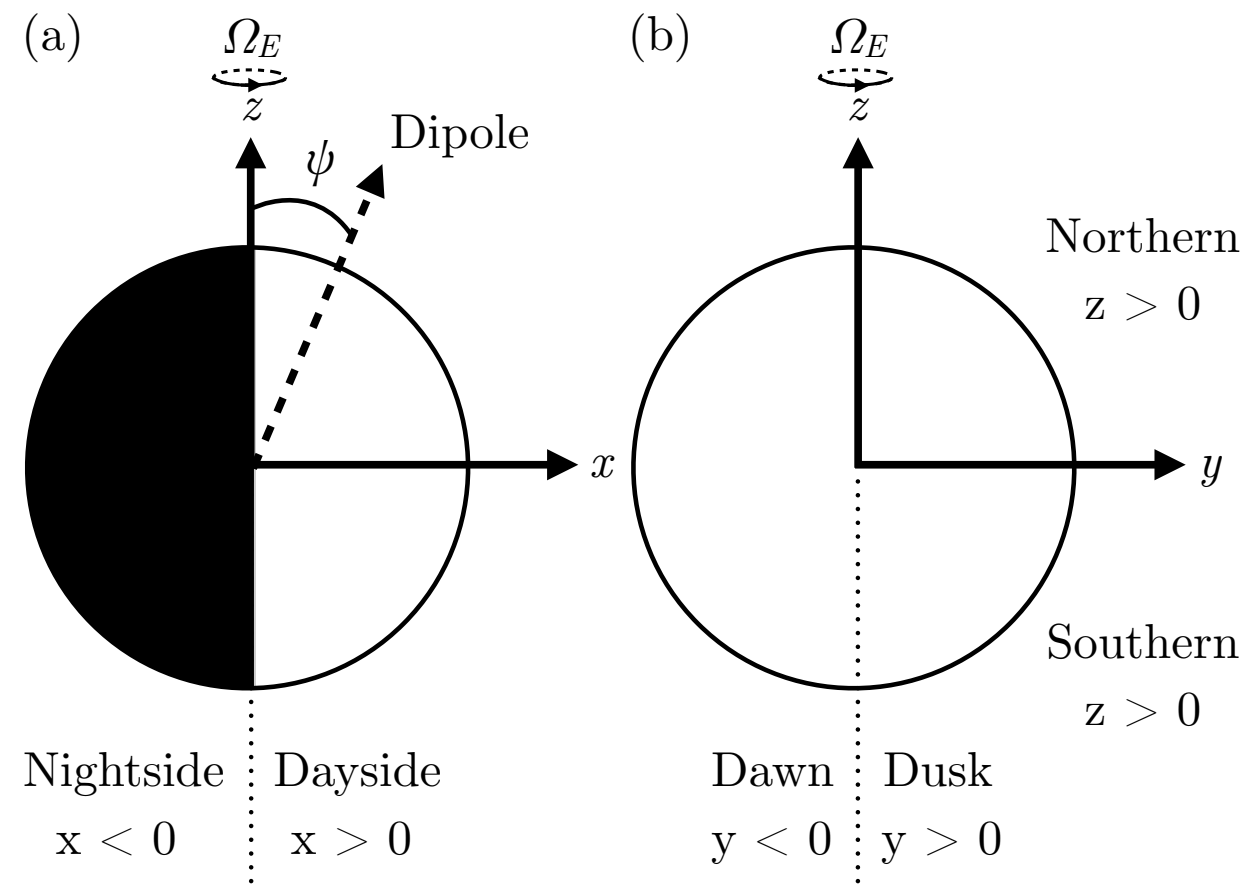

Figure 1.2: Schematic diagram of the Geocentric Solar Magnetic (GSM) coordinate system. Panel (a) looks along the $+y$ axis and (b) looks Earthward from the sun along the $-x$ axis. The $z$ axis is perpendicular to Earth's orbital plane, the $x$ axis points from the Earth to the sun, and the $y$ axis completes the right-handed triplet. Earth's magnetic dipole is depicted with the dashed black line; the magnetic dipole tilt angle $\psi$ is confined to the $x$ - $z$ plane where $\psi>0$ has the northern geomagnetic pole pointing sunward.

oriented along the $z$ GSM axis; the rotational axis is oriented with the $z$ axis in Fig. 1.2 only to demonstrate dawn and dusk on Earth's surface. Distances are typically given in Earth $\operatorname{radii}\left(R_{\mathrm{E}}\right)$, which averages $6,371 \mathrm{~km}$.

Two other coordinates that are of use to orbiting satellites are Magnetic Local Time (MLT) and geomagnetic latitude (Lat). MLT is analogous to geographic longitude on Earth's surface, and gives the spacecraft's location in the $x-y$ GSM plane in hh:mm as measured from the $-x$ GSM axis, or midnight. Therefore, a spacecraft at 09:00 MLT is located at $45^{\circ}$ between the $+x$ and $-y$ GSM axes. Geomagnetic latitude is the angle measured from the magnetic equator, i.e., the plane at Earth's center which is normal to the magnetic dipole, with $+90^{\circ}$ corresponding to the northern geomagnetic pole and $-90^{\circ}$ for the southern 

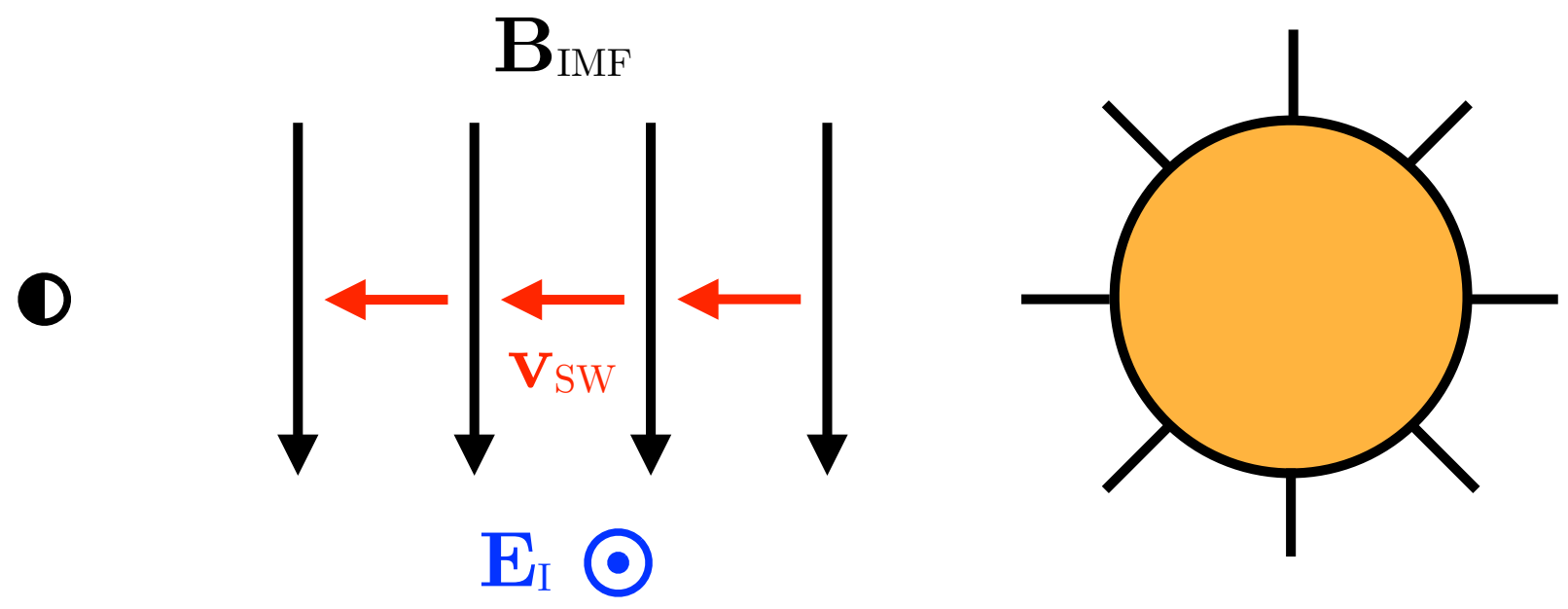

Figure 1.3: Schematic diagram of the solar wind bulk flow velocity $\mathbf{v}_{\mathrm{SW}}$ flowing outward from the sun (right) towards Earth (left). The solar wind carries the interplanetary magnetic field $\mathbf{B}_{\text {IMF }}$, which sets up the interplanetary electric field $\mathbf{E}$. Depicted features are not to scale.

geomagnetic pole.

Interplanetary space is filled with a stream of plasma constituted of electrons and predominantly hydrogen ions emanating from the sun, called the solar wind (Parker, 1958). The solar wind flows outward from the sun, carrying with it a magnetic field. This flow and magnetic field are associated with an electric field, called the interplanetary electric field $\mathbf{E}_{\mathrm{I}}$. This is given by the ideal Ohm's law

$$
\mathbf{E}=-\mathbf{v} \times \mathbf{B}
$$

where $\mathbf{v}$ is the bulk flow velocity and $\mathbf{B}$ is the magnetic field. Therefore, $\mathbf{E}_{\mathrm{I}}=-\mathbf{v}_{\mathrm{SW}} \times \mathbf{B}_{\mathrm{IMF}}$, where $\mathbf{v}_{\mathrm{SW}}$ is the solar wind velocity and $\mathbf{B}_{\mathrm{IMF}}$ is the interplanetary magnetic field (IMF). Figure 1.3 displays a schematic diagram of the solar wind flow and the IMF which sets up this electric field.

Several days after leaving the sun, the solar wind arrives at Earth. In the GSM coordinate system, the solar wind flows predominantly in the $-x$ direction, although it can have compo- 
nents along the other two axes. The IMF can have an arbitrary orientation at 1 astronomical unit (AU), the mean distance between the sun and the Earth measuring almost $1.5 \times 10^{8} \mathrm{~km}$ $\left(\approx 24,000 \mathrm{R}_{\mathrm{E}}\right)$. The IMF can be completely described by its $B_{x}$ component, magnitude $B_{\mathrm{IMF}}$, and clock angle $\theta_{\mathrm{IMF}}$ which is the angle the IMF makes with the $+z$ axis as measured in the $y$ - $z$ plane. Solar wind plasma parameters measured at 1 AU are solar wind flow speed $v_{\mathrm{SW}}=400-1500 \mathrm{~km} / \mathrm{s}$, interplanetary magnetic field strength $B_{\mathrm{IMF}} \sim 1-10 \mathrm{nT}$, plasma number density $n_{\mathrm{SW}} \sim 1-10 \mathrm{~cm}^{-3}$, and temperatures $T_{\mathrm{SW}} \sim 10^{5}-10^{6} \mathrm{~K}(\sim 10-100 \mathrm{eV})$; the lower limits of these ranges are representative of the more common, or "quiet" solar wind [see Gonzalez et al. (1999), and references therein]. (We use $\sim$ here to denote "on the order of" to convey the order of magnitude in these parameters.)

As the solar wind approaches the Earth, the dipolar geomagnetic field exerts its influence on the solar wind plasma. The geomagnetic field acts as an obstacle, which creates a bow shock typically $15 \mathrm{R}_{\mathrm{E}}$ upstream of Earth. For quiet solar wind conditions, this interaction slows the solar wind speed by a factor of 3-4, whereas the plasma density and IMF strength increase by the same factor. The plasma temperature increases as well, but with different scaling. The Earthward side of the bow shock is known as the magnetosheath, in which the "shocked" solar wind continues to move towards Earth until it encounters the magnetosphere, the region of space dominated by Earth's magnetic field. The boundary of the magnetosphere is the magnetopause, a bullet-shaped magnetic obstacle that carries a current density due to differences in the magnitude and orientation between the magnetosheath and geomagnetic fields; the subsolar point is the magnetopause's furthest extent towards the sun, typically located around $10 \mathrm{R}_{\mathrm{E}}$ under quiet solar wind conditions. The solar wind's continuous flow past Earth stretches the nightside magnetic field out to form an elongated magnetotail, which can extend $\sim 10^{2}-10^{3} \mathrm{R}_{\mathrm{E}}$ downstream of Earth. 

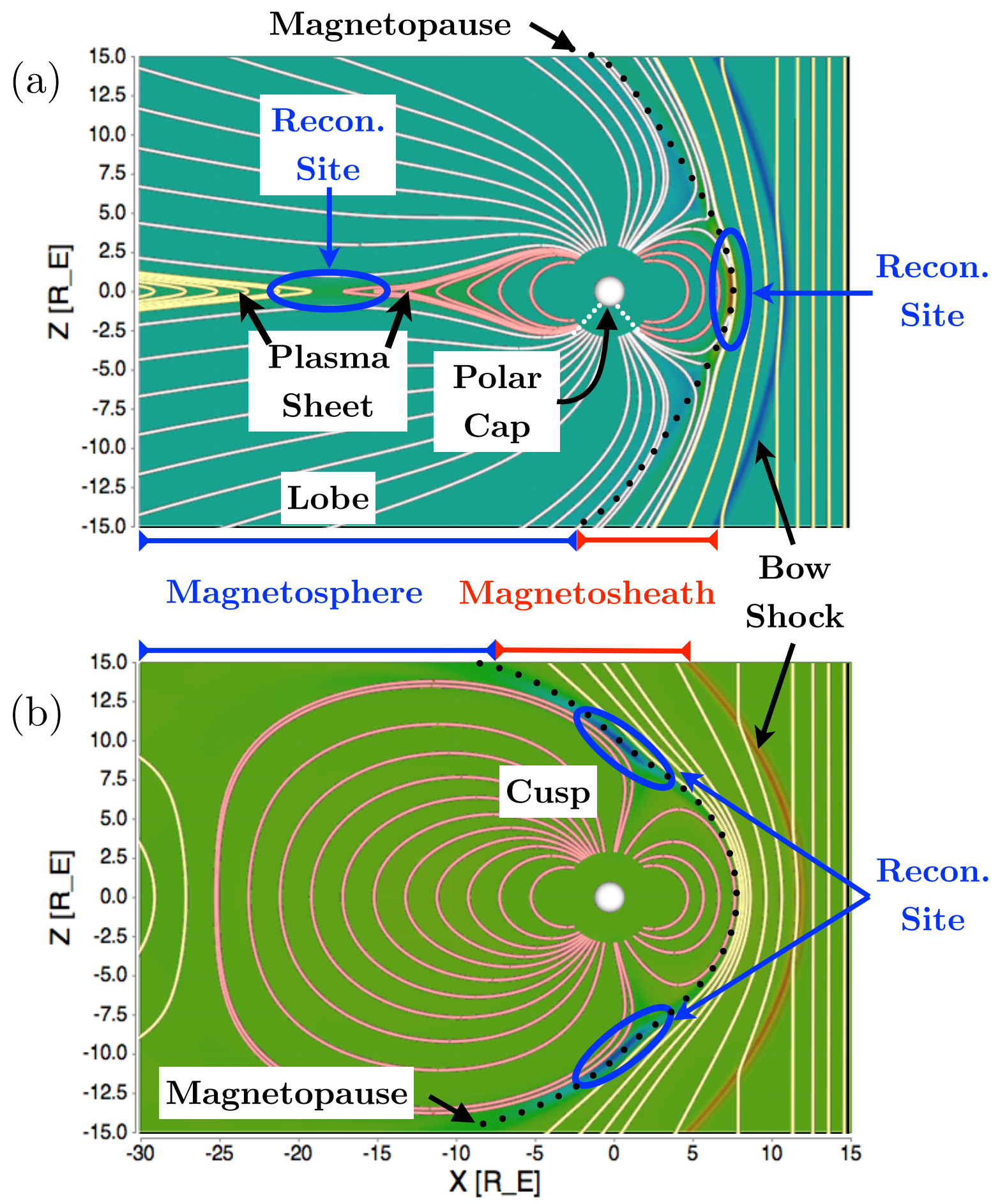

Figure 1.4: Diagram of Earth's magnetosphere with geomagnetic field lines depicted in red and interplanetary magnetic field lines colored yellow with (a) southward and (b) northward orientations from the $y=0$ GSM plane of simulations to be described in Chapter 2. The current density $J_{y}$ is the color background with blue pointing out of the page $\left(J_{y}<0\right)$ and red into the page $\left(J_{y}>0\right)$. Reconnection occurs between the closed geomagnetic field lines (red lines) and the IMF (yellow lines) within the blue ovals and panel (a) displays open magnetic field lines in gray. The approximate location of the magnetopause is sketched with the dotted black line. 
These features of the near-Earth space environment are depicted in Fig. 1.4. In both figures, taken from simulations to be described in Chapter 2, the solar wind flows in from the right bringing due (a) southward $\left(B_{z}<0\right)$ and (b) northward $\left(B_{z}>0\right)$ IMF. The colored background depicts the current density $J_{y}$ in GSM coordinates, where blue is negative (out of the page) and red is positive (into the page). In both figures, the IMF becomes noticeably curved in the magnetosheath after crossing the bow shock represented by the current layers on the right. One can step out parallel and antiparallel along a magnetic field, i.e., $b i$ directionally trace, in order to map field lines. IMF lines do not map to Earth and are colored yellow, whereas closed geomagnetic field lines within the magnetosphere map to both geomagnetic poles and are colored red. The magnetopause is located between the rightmost closed geomagnetic and the leftmost interplanetary magnetic field lines and is sketched with the dotted black line. Open magnetic field lines map to one of the geomagnetic poles and are colored gray in Fig. 1.4(a).

Features of the magnetotail during southward IMF conditions are depicted in Fig. 1.4(a). The thin plasma sheet is approximately located in the magnetotail's mid-plane and contains a current that points into the page. The magnetospheric lobes are located several $\mathrm{R}_{\mathrm{E}}$ vertically above and below the plasma sheet and contain open magnetic field lines depicted in gray.

For the case of northward IMF in Fig. 1.4(b), the magnetosheath plasma is largely deflected around the magnetopause and away from Earth, with minimal plasma entry into Earth's magnetosphere. The magnetospheric cusps are displayed in Fig. 1.4(b) located near the bluish-purple current layers directly above and below the northern and southern geomagnetic poles. These cusps can be identified by where the magnetic field emanating from the southern geomagnetic pole abruptly changes direction, extending to the distant magnetotail on one side and to the dayside on the other; similar features can be seen at the 
northern magnetic cusp with the only differences being the geomagnetic field comes from the magnetotail and magnetopause and returns to the northern geomagnetic pole.

Much closer to Earth, the atmosphere we commonly experience on the surface slowly transitions to the plasma environment of space. In between these distinct boundaries, ultraviolet light from the sun ionizes (photo-ionizes) neutral particles to create plasma which constitutes the ionosphere $\sim 100 \mathrm{~km}\left(\sim 0.01 \mathrm{R}_{\mathrm{E}}\right)$ above Earth's surface. Collisions occur between ionospheric plasma particles and result in interesting electrodynamics of the ionosphere determined by the ionosphere's electromagnetic environment. These collisions set up an electric field $\mathbf{E}$ related to ionospheric currents by

$$
\mathbf{J}=\boldsymbol{\sigma} \cdot \mathbf{E}
$$

where $\mathbf{J}$ is the ionospheric current density and $\boldsymbol{\sigma}$ is the conductivity tensor which describes how readily the plasma can move in an electric field. The conductivity tensor consists of three primary conductances: (1) the Pederson conductance $\sigma_{P}$ is the conductance in the direction of the ionospheric electric field, (2) the Hall conductance $\sigma_{H}$ is the conductance perpendicular to both the ionospheric electric and magnetic fields, and (3) the parallel conductance $\sigma_{\|}$which is the conductance along the magnetic field lines, which are nominally ideal conductors with minimal impedance of plasma movement along geomagnetic field lines. The open - closed boundary defines the polar cap at high geomagnetic latitudes, where the interior of which contains all of the open magnetic field lines (Kivelson and Russell, 1995; Kelley, 2009; Baumjohann and Treumann, 2012).

Significant differences between northward and southward IMF result from magnetic reconnection, which occurs within the blue ovals depicted in Fig. 1.4. Reconnection's role in 
the dynamics of Earth's magnetosphere is discussed in the next section.

\subsection{Magnetospheric Reconnection}

Magnetic reconnection occurs in plasmas where magnetic field lines with oppositely directed components merge, effectively break, and subsequently stitch together or "reconnect." An example of one place where reconnection occurs is the dayside magnetopause displayed with the blue oval on the right in Fig. 1.4(a). Here the oppositely directed southward IMF meets the northward oriented geomagnetic field at the subsolar point. The reconnection process, which will be described in greater detail in Section 1.4, results in open magnetic field lines that are strongly bent at the reconnection site, as seen if Fig. 1.4(a). These open field lines slingshot away from the reconnection site acting like stretched rubber bands that straighten out upon release. This process converts stored magnetic energy in the reconnecting magnetic fields into plasma kinetic energy and heat. This merging process at the dayside magnetopause allows solar wind plasma to transfer into Earth's magnetosphere.

Reconnection is not limited to the dayside magnetopause; as seen in Fig. 1.4, the solar wind bulk flow pulls the reconnected magnetic field lines anti-sunward, causing them to move towards the magnetotail. These magnetic fields accumulate in the magnetospheric lobes. As time goes on, magnetic flux accumulates, squeezing the magnetic field towards the plasma sheet. These open field lines are oppositely directed and, once a threshold that is not well understood is exceeded, they explosively undergo magnetic reconnection again. They form IMF and closed magnetic field lines, as depicted on the left of Fig. 1.4(a). Magnetotail reconnection moves energetic plasma Earthward where it can penetrate near-Earth space and cause space weather related problems like those discussed at the beginning of this chapter, 
such as possibly interfering with telecommunication satellites and also precipitating down into Earth's atmosphere to generate the beautiful aurorae. For sustained southward IMF conditions, newly formed geomagnetic field lines move Earthward, where they are redirected around the Earth, that is, in the out-of-plane direction in Fig. 1.4, and return to the dayside magnetopause where they can undergo reconnection again.

The process of the geomagnetic field moving from the dayside magnetopause to magnetotail and back is referred to as magnetospheric convection, or the Dungey cycle, named after James Dungey who originally proposed the model (Dungey, 1961, 1963). Dungey (1961) argued that the convection of magnetic field lines through the magnetosphere has an observable signature at polar latitudes of Earth. Turning back again to Fig. 1.4, after reconnection occurs at the subsolar point open magnetic field lines move tailward. The open field lines traveling tailward in the $y=0$ GSM plane leave an imprint on the polar cap, where the footpoints of these open magnetic fields also move in the $-x$ direction. At the geomagnetic north pole, the magnetic field is predominantly in the $-z$ direction which sets up an ideal electric field given by Eq. (1.1) that points in the $+y$ direction. This electric field can be interpreted as a potential difference, where the electric potential $\phi$ is given by

$$
\mathbf{E}=-\nabla \phi
$$

After reconnection occurs in the magnetotail, the newly formed geomagnetic field lines move Earthward (in the $+x$ direction), and attain a finite $y$ velocity component as they convect around Earth back to the dayside magnetopause. This potential is measurable giving observational evidence for the model [see Boudouridis et al. (2005), and references therein].

Figure 1.5(a) displays the polar cap in a simulation with southward IMF. In this figure, 

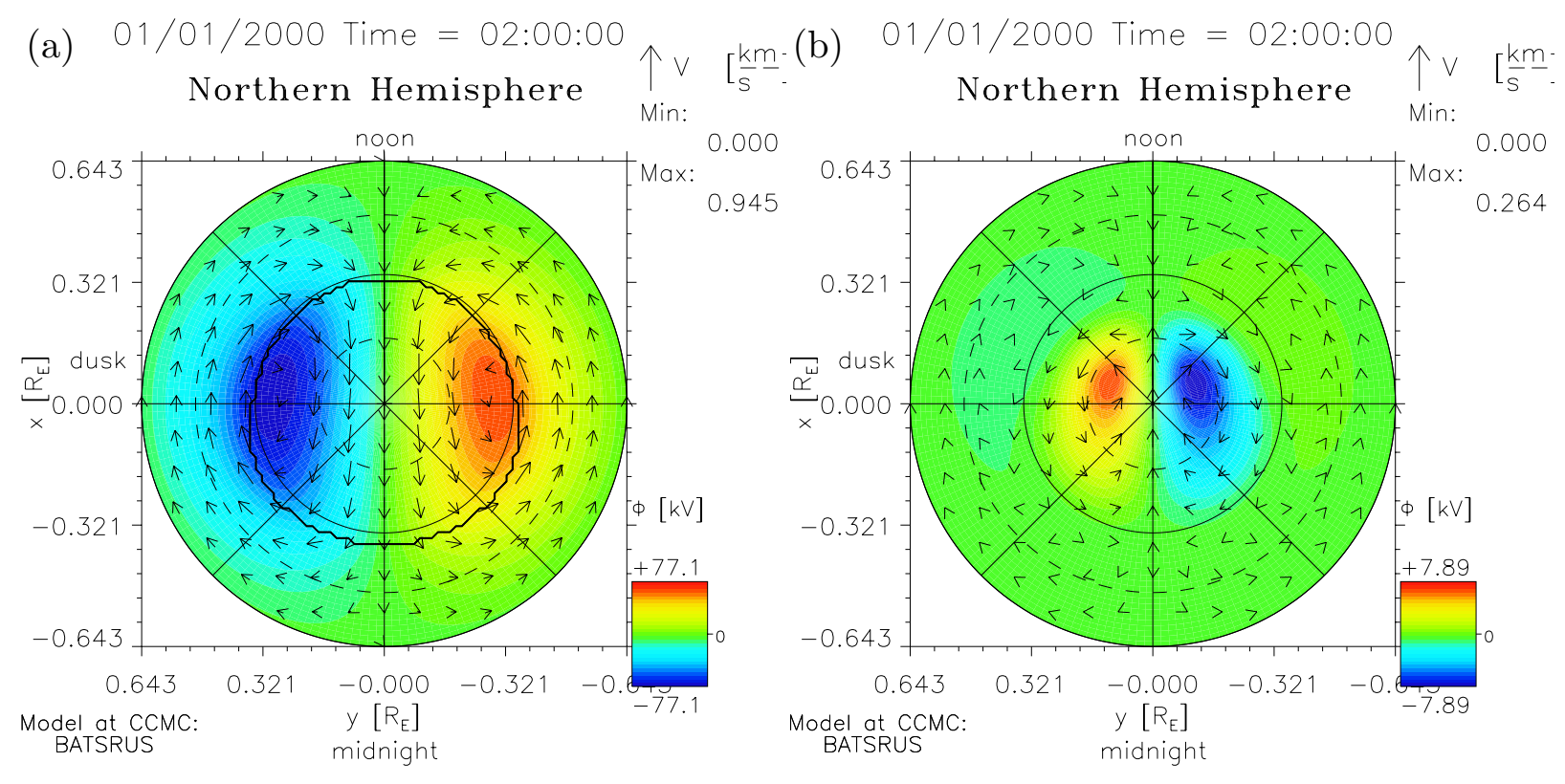

Figure 1.5: The electric potential calculated at the northern polar cap in simulations with (a) southward and (b) northward IMF. The colored background displays the electric potential $\phi$ and the inplane flow patterns of the ionospheric magnetic field lines. The solid black circular line in (a) displays the polar cap boundary, the interior of which contains all of the open/reconnected field lines.

the velocity $\mathbf{v}$ of the ionospheric plasma is displayed with arrows, and the magnetic field $\mathbf{B}$ points into the page, which gives rise to an electric field $\mathbf{E}$ pointing from dawn to dusk in the noon-midnight plane, i.e., pointing from right to left near $y=0$. The electric potential $\phi$ is calculated on the polar cap and is plotted as the color background. The tailward flow in the noon-midnight plane followed by flow towards dusk and dawn is the ionospheric signature of the Dungey cycle.

While most of the discussion so far has focused primarily on southward IMF conditions, Dungey (1961) also presented a model for when the IMF is directed due northward and without any magnetospheric tilt. In this case reconnection occurs at the magnetospheric cusps, as shown in Fig. 1.4(b). In this idealized case, reconnection produces a single closed and solar wind field line without any transition to open magnetic field lines, thus reconnection is less efficient at coupling solar wind plasma into Earth's magnetosphere. The polar cap 
for northward IMF is shown in Fig. 1.5(b). Although the polar cap potential patterns for northward IMF and southward IMF conditions look very similar in Fig. 1.5, there are some noteworthy differences. The first is that no open-closed boundary can be seen under northward IMF conditions unlike that seen under southward IMF, depicted as the the solidblack circle in Fig. 1.5(a). Reconnection for northward IMF results from the IMF draping across the magnetopause where it simultaneously reconnects at both magnetospheric cusps without transitioning to an intermediate open topology, as seen in Fig.1.4(b), so there is no open-closed magnetic field boundary.

Another difference is the magnitude of the potential in the two cases. This is quantified by defining the cross polar cap potential difference $\Delta \Phi_{P C}$, which is given by the difference between the maximum and minimum values of the ionospheric potential $\phi$ and calculated as

$$
\Delta \Phi_{P C}=\max (\phi)-\min (\phi)
$$

This is considerably smaller for northward IMF $(16 \mathrm{kV})$ than that measured for southward $\operatorname{IMF}(154 \mathrm{kV})$.

Finally, we note that the convection patterns of the geomagnetic field seen in the polar cap point in opposite directions, which results from it being caused by a completely different mechanism for northward IMF. In particular, the convection cells for northward IMF result from what is known as the viscous interaction of the geomagnetic field with the IMF as it flows around the magnetopause, and a schematic diagram of this magnetospheric convection pattern is shown in the left half of Fig. 1.6. The solar wind's flow around the magnetopause produces a velocity gradient across the magnetopause, giving rise to viscous effects. This flow around the magnetosphere sets up eddy flows whereby magnetic fields just inside of 

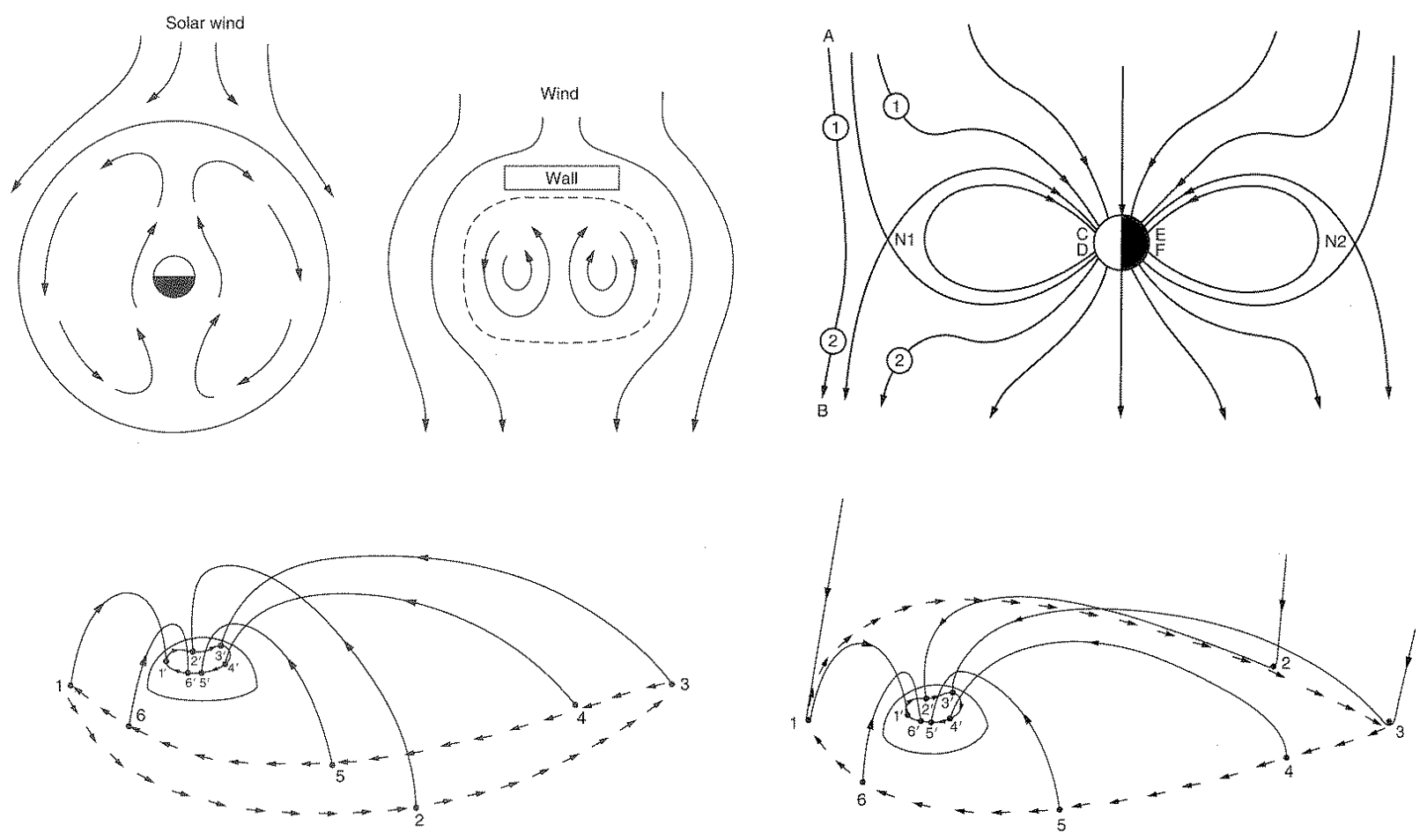

Figure 1.6: Schematic diagram of magnetospheric convection patterns resulting from (left) the viscous interaction and (right) magnetic reconnection under northward and southward IMF conditions, respectively. The numbers 1 through 6 display the time history of a particular magnetic field line. Reprinted from M. C. Kelley (2009) The Earth's Ionosphere: Plasma Physics and Electrodynamics: Second Edition, pp. 408-409, Academic Press. Copyright 2009, with permission from Elsevier Inc.

the magnetopause flow tailward. Once these geomagnetic fields reach the tail, they then convect back towards the magnetopause along the $+x$ direction in the $y=0$ GSM plane.

We note that magnetospheric convection resulting from the viscous interaction is in the exact opposite direction as the convection pattern resulting from reconnection as predicted by Dungey $(1961,1963)$ and is sketched in the right half of Fig. 1.6. We note that the potential patterns from viscosity and reconnection are very different, as seen in Fig. 1.5; the negative potential cell occurs in the (a) dusk sector $(y>0)$ and (b) dawn sector $(y<0)$ for southward and northward IMF, respectively.

In concluding this discussion, the electric potential patterns at the polar cap also present 
an important observational signature for measuring reconnection's role in magnetospheric convection. The larger the polar cap potential difference, the more efficiently reconnection at the dayside occurs. However, $\Delta \Phi_{P C}$ has been shown to "saturate," meaning that $\Delta \Phi_{P C}$ no longer increases proportionally to the IMF strength $B_{\mathrm{IMF}}$ above some threshold. This has been used as evidence that reconnection becomes less efficient at converting IMF with large strengths that encounter the dayside magnetopause [see Lopez et al. (2010), and references therein]. Finally, the size of the open-closed boundary, which defines the edge of the polar cap, has been used as a measure of the reconnection rate, as a growing open magnetic flux boundary indicates reconnection is penetrating deeper into Earth's magnetosphere [see Boudouridis et al. (2005), and references therein].

\subsection{Reconnection's Importance for Space Weather}

Making contact with the examples given at the beginning of this chapter, reconnection has a direct role affecting space weather and how astronauts and satellites can be affected by solar storms. The prevailing consensus is that reconnection is the mechanism that initiates solar flares and result in CMEs being released into interplanetary space where astronauts can be irradiated with energetic solar protons. The aforementioned loss of the Galaxy IV satellite is largely assumed to have resulted from energetic electrons in the inner magnetosphere that resulted in spacecraft charging, causing electric discharges that fried critical components of the satellites internal circuitry (Baker et al., 1998). There are many different avenues

by which these "killer electrons" become energized in the inner magnetosphere which are consequences of reconnection's role in solar wind-magnetosphere coupling.

Reconnection at the dayside magnetopause or in the magnetotail can directly affect three 
areas of the inner magnetosphere: the plasmasphere, the radiation belts, and the ring current. Although these three regions overlap for large portions of the inner magnetosphere, they have largely been treated as distinct systems due to historical limitations in observations. However, the field is in the process of developing a holistic understanding of the feedback mechanisms among these magnetospheric "subsystems" to aid in space weather predictions, where the dynamics of one system can have very important effects on adjacent domains (Borovsky et al., 2013a).

\subsubsection{Reconnection's Role in Plasmaspheric Plasma Transport}

The plasmasphere is effectively a continuation of the ionosphere, extending out to a few $R_{E}$. In general, the plasmasphere consists of cold $\left[T \sim 10^{3}-10^{4} \mathrm{~K}\right]$, high density $\left[n \sim 10^{2} \mathrm{~cm}^{-3}\right]$ plasma that extends out to the plasmapause around $4 \mathrm{R}_{\mathrm{E}}$ where the density precipitously drops to $n \sim 10 \mathrm{~cm}^{-3}$ (Baumjohann and Treumann, 2012).

The dayside interaction between Earth's magnetosphere and the solar wind can have profound effects on the plasmasphere, even though it is located relatively far from the dayside magnetopause. For example, under prolonged northward conditions like those shown in Fig. 1.4(b), reconnection is at a minimum allowing for the plasmasphere to expand radially outward as it continuously fills up with cold, dense plasma. A sudden turning of the IMF to a southward orientation causes reconnection to occur near the subsolar point, pulling in plasma from the magnetosphere. The plasmapause develops a plasmaspheric drainage plume,

a plasmaspheric "tendril" that can extend all the way to the magnetopause (Borovsky, 2014; Walsh et al., 2014). This cold, dense plasmaspheric plasma mass-loads the magnetosphere at the reconnection site, which as we will see in Section 1.4 .2 causes reconnection to slow down in response to the increased mass. The expansion of the plasmasphere plays an impor- 
tant role in slowing the entry of solar wind plasma into Earth's magnetosphere, where this effect can slow magnetospheric convection and possibly decrease the severity of geomagnetic storms (Borovsky and Steinberg, 2006).

\subsubsection{Outer Radiation Belt Losses Resulting from Dayside Recon- nection}

The radiation belts, also known as the Van Allen belts, are two toroidal structures whose axes are aligned with the geomagnetic dipole that encircle Earth. The relatively stable inner radiation belt is located between 1.2-2.0 $\mathrm{R}_{\mathrm{E}}$ and the dynamic outer radiation belt occupies a much larger region from $2.5-7 \mathrm{R}_{\mathrm{E}}$. The inner radiation belt consists predominantly of relativistic ions $>10 \mathrm{MeV}$ and energetic electrons with energies between $0.04-1 \mathrm{MeV}$, whereas the outer belt contains relativistic electron populations with energies $>1 \mathrm{MeV}$ and energetic ions with energies $\sim 0.1 \mathrm{MeV}$ (Baumjohann and Treumann, 2012).

Particles in the radiation belts exhibit three characteristic motions: (1) gyro-motion about the geomagnetic field, (2) a bounce motion along the geomagnetic field, and (3) an azimuthal drift around Earth. The timescales of these motions are $\sim 10^{-3} \mathrm{~s}, \sim 10^{0} \mathrm{~s}$, $\sim 10^{3} \mathrm{~s}$, respectively, for a $1 \mathrm{MeV}$ electron at geosynchronous orbit $\left(6.6 \mathrm{R}_{\mathrm{E}}\right)$ with a $60^{\circ}$ pitch angle, i.e., the angle its velocity vector makes with the magnetic field. So long as the electromagnetic environment of the inner magnetosphere changes more slowly than the timescale of the motion, these motions are largely steady and associated with conserved quantities referred to as adiabatic invariants. However, the electromagnetic environment of Earth's magnetosphere gives rise to many different kinds of waves which can interact with normally stable particles and result in their loss from the outer belt. Throughout this 
discussion, we refer to particles as "lost" if they are no longer confined to closed geomagnetic field lines.

Stably trapped radiation belt electrons normally lie on closed drift shells, called $L$-shells, defined by a particles' conserved azimuthal drift (Shabansky, 1971). However, a known loss mechanism of radiation belt populations is 'magnetopause shadowing,' where particles are lost to interplanetary space if they make contact with the magnetopause and jump from geomagnetic field lines to a solar wind field line.

Another loss mechanism that has long been theorized to contribute to outer belt losses is the interaction of relativistic electrons with electromagnetic ion cyclotron [EMIC] waves. In this wave-particle interaction, waves traveling parallel to the geomagnetic field can synchronize with an electron's gyro-motion about the magnetic field, resulting in electron energization [see Thorne (2010), and references therein]. This energization can decrease the electron's pitch angle so that its velocity is nearly parallel to the magnetic field. This pitch angle scattering allows the electron to travel further along the magnetic field line, and if it encounters the ionosphere, the electron can be lost to Earth's atmosphere due to collisions.

There are at least two mechanisms by which dayside reconnection can lead to radiation belt losses. The first of which results from reconnection's direct role in determining the location of the magnetopause. For southward IMF, the magnetopause location is at smaller radial distances for increasingly large and negative $B_{z}$ (Lu et al., 2011). If $B_{z}$ is negative and decreasing, the inward movement results in the "erosion" of the geomagnetic field from reconnection, which increases the likelihood of magnetopause shadowing. This is particularly strong for impulsive solar storms with southward IMF (Turner et al., 2012).

The second mechanism indirectly involves reconnection. We return again to the scenario of prolonged northward IMF periods followed by a sudden turning of the IMF to a southward 


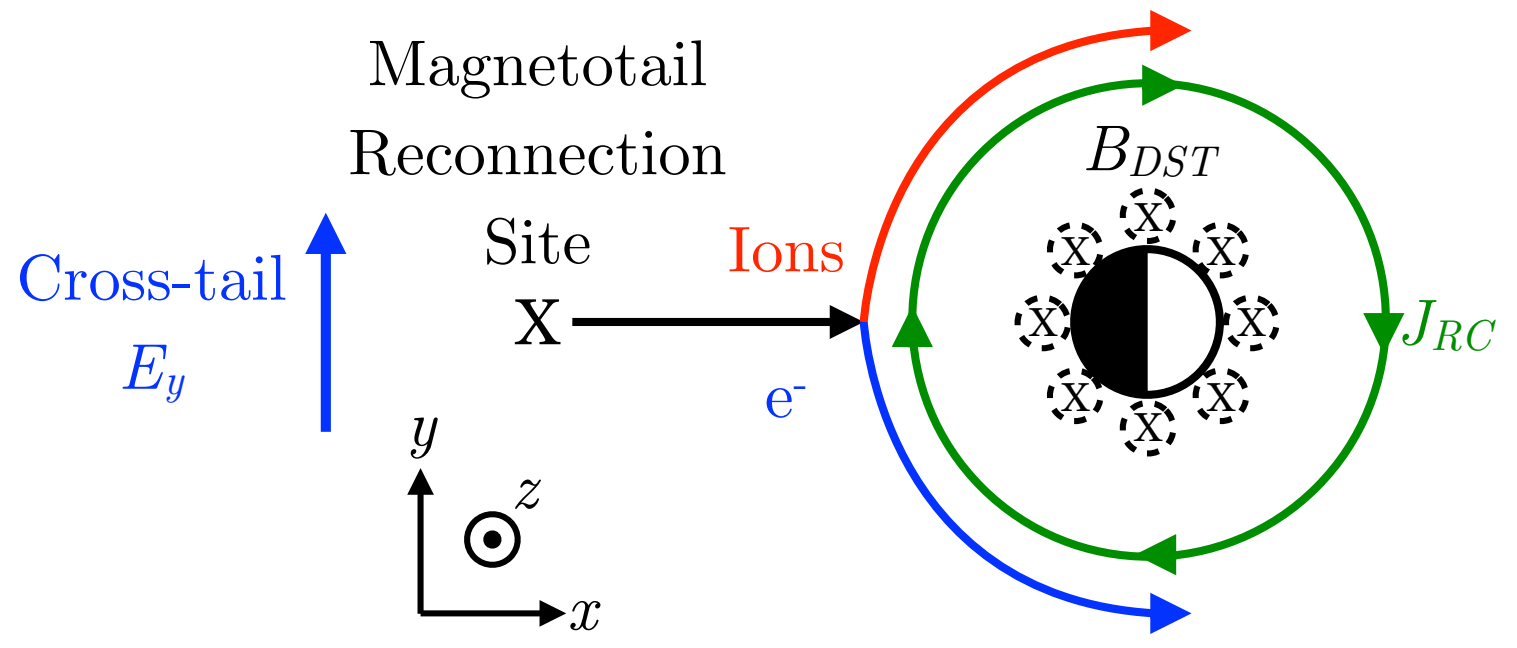

Figure 1.7: Schematic diagram of Earth's ring current in the geomagnetic equatorial plane. Reconnection occurs in the magnetotail, depicted with an $\mathrm{X}$ on the left, and generates a cross-tail electric field $E_{y}>0$ which convects the plasma Earthward. At about $3 \mathrm{R}_{\mathrm{E}}$, the ions and electrons begin drifting in different directions around the Earth; the ions (top, red path) drift westward, i.e., counterclockwise in this figure. This charge separation creates the ring current $J_{R C}$ (green), which in turn creates a magnetic field $B_{D S T}$ (dashed circles) that opposes Earth's surface magnetic field $\left(B_{z}>0\right)$.

orientation. This "calm before the storm" initializes the magnetosphere in a low energy state, allowing for the plasmasphere to refill with very dense, low temperature plasma, ideal conditions for generating EMIC waves across the plasmasphere's edge (Jordanova et al., 2007; Chen et al., 2010; Pickett et al., 2010). Once the IMF turns southward, a plasmaspheric drainage plume can develop, extending the plasmasphere across the outer radiation belt. EMIC waves at the plume's edge can interact with relativistic electrons in the outer belt where substantial losses of these radiation belt populations have been observed (Thomsen et al., 2003; Borovsky and Steinberg, 2006; Lavraud et al., 2006; Borovsky and Denton, 2009).

\subsubsection{Ring Current Energization from Magnetotail Reconnection}

Reconnection in the magnetotail results in a cross-tail electric field pointing in the $+y_{\mathrm{GSM}}$ direction, i.e., into the page in Fig. 1.4(a) for southward IMF. The electric fields generated 
by this reconnection process convect plasma Earthward on the newly reconnected geomagnetic field lines. As this plasma approaches Earth, it feels the influence of the electric field generated by magnetic fields co-rotating with Earth. At sufficiently small radial distances, the plasma on these newly reconnected geomagnetic field lines ceases to move Earthward and begins rotating around Earth, with ions rotating westward and low energy electrons traveling eastward; this separation and movement of plasma species generates the ring current which occupies radial distances of several $\mathrm{R}_{\mathrm{E}}$ (Baumjohann and Treumann, 2012).

Figure 1.7 displays many of the important features of the ring current in the geomagnetic equatorial plane. Reconnection occurs at the $\mathrm{X}$ on the left in the distant magnetotail and convects the plasma Earthward. The ions (red path) and electrons (blue path) are shown to separate close to Earth which sets up the ring current's current density $J_{R C}$.

The convection of plasma from the distant magnetotail has profound changes to a particle's energy distribution. To demonstrate this, let us consider the case of plasma tied to a newly reconnected geomagnetic field line on the Earthward side of the magnetotail reconnection site with equal amounts of thermal energy parallel and perpendicular to the magnetic field, i.e., the plasma is isotropic. As this isotropic plasma convects Earthward, the geomagnetic field strength increases since Earth's dipolar magnetic field is inversely proportional to distance. As the plasma enters this stronger magnetic field, it gyrates around the magnetic field faster, thus increasing its perpendicular thermal energy. In order to conserve its total energy, the particle's parallel thermal energy is transferred to its perpendicular energy. Thus, the plasma has become anisotropic as part of its convection towards Earth. The development of anisotropic ion populations in the ring current has long been known to efficiently generate EMIC waves throughout the magnetosphere's dusk sector (Cornwall, 1965; Kennel and Petschek, 1966), which can result in pitch angle scattering of outer belt electrons as 
previously discussed.

Finally, the energy of the ring current has another important effect on Earth. The ring current is appropriately named as this structure encircles the Earth with the bulk of the plasma located near the geomagnetic equator. As depicted in Fig. 1.7, the ring current is similar to a current passing through a circular wire, where the westward directed current generates a magnetic field $B_{D S T}$ which opposes the geomagnetic field, resulting in measurable changes to Earth's surface magnetic field. The disturbance storm time (Dst) geomagnetic index is used to determine an impulsive solar storm's onset, severity, and duration. For southward IMF conditions, the $D$ st index typically has values of $-10>$ Dst $>-150 \mathrm{nT}$ and sometimes even lower values during the most severe solar storms. The sudden increase of ring current energy can have detrimental effects at Earth's surface where the impulsive onset of these magnetic depressions can create currents in terrestrial transmission lines, as evidenced by the aforementioned destruction of electrical infrastructure in Québec in March 1989. The measured $D s t$ for this particular solar storm was almost $-600 \mathrm{nT}$ at the storm's peak, roughly $2 \%$ of Earth's average surface magnetic field strength.

In summary, reconnection plays an important role in the composition and dynamics of the inner magnetosphere. Due to the unpredictable and highly dynamic nature of the solar wind, Earth's magnetosphere can have a variety of different responses for seemingly similar solar wind conditions. Research is ongoing to understand the causal relationship between reconnection and its influence on magnetospheric dynamics. Therefore, obtaining a more thorough understanding of reconnection throughout Earth's magnetosphere is necessary for achieving reliable space weather predictions. 


\subsection{The Local Physics of Magnetic Reconnection}

The simplest description of magnetic reconnection occurs in plasmas between oppositely directed magnetic fields where the stored magnetic energy of the magnetic fields is converted into plasma kinetic energy and heat. In the following sections, general features of antiparallel reconnection in a two-dimensional plane are discussed, like the reconnection between the northward geomagnetic and the southward interplanetary magnetic fields depicted at the subsolar magnetopause in Fig. 1.4(a).

\subsubsection{Symmetric Reconnection}

Figure 1.8 depicts the reconnecting magnetic fields local to the reconnection site. Upstream (vertically above and below) of the dotted box, the plasma has mass density $\rho$ and is threaded with a magnetic field of strength $B_{0}$ where it flows in with velocity $v_{i n}$. The movement of this "ideal" plasma is associated with an out-of-plane convective electric field $E_{\text {conv }}$ is

$$
E_{\text {conv }} \sim v_{\text {in }} B_{0}
$$

resulting from the ideal Ohm's law given by Eq. (1.1). In contrast, inside of the box, the plasma decouples from the magnetic field resulting from non-ideal effects such as collisions (resistivity), electron inertia, or finite Larmor radius effects such as the Hall effect. The entire process of magnetic reconnection, i.e., the change in a field line's topology, occurs within the depicted box; this box is called the diffusion region.

Internal to the diffusion region, two points of interest are the stagnation point (S) and magnetic $\mathrm{X}$-line $(\mathrm{X})$, and are seen in the middle plot of Fig. 1.8. The stagnation point marks the location where the inflowing plasma meet and come to a halt. The X-line is the location 


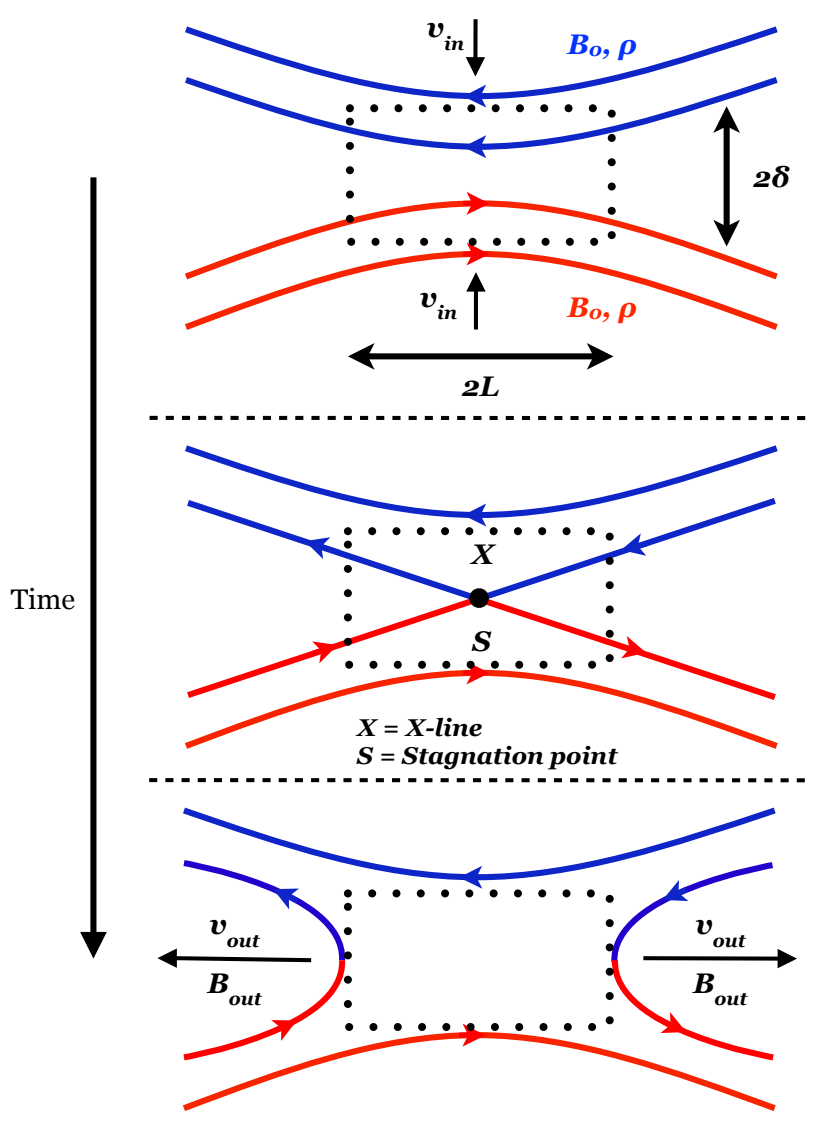

Figure 1.8: Schematic diagram of reconnection occurring between plasmas of density $\rho$ and magnetic fields with equal and opposite strengths $B_{0}$ at a time corresponding to (top) before, (middle) the commencement of, and (bottom) after reconnection. The X-line (X) and stagnation point (S) are located at the exact center of the diffusion region (black dotted box) with half-length $L$ and half-width $\delta$. Reconnection occurs at the X-line and the plasma flows horizontally outward of the dissipation region at speed $v_{\text {out }}$. Edited with permission from C. Doss.

where the reconnecting magnetic field is zero, marking where the magnetic topology changes; the X-line gets its name from the shape of the magnetic field lines at the moment reconnection occurs. These field lines get close enough to each other where they ultimately break, and cross-connect with each other. For symmetric reconnection, the X-line and stagnation point are at the same location in the center of the diffusion region.

Newly reconnected magnetic field lines are strongly bent at the X-line. These bent field 
lines have a large magnetic tension force causing them to straighten out in the horizontal direction, analogous to a stretched rubber band. From magnetohydrodynamic (MHD) theory, to be described in greater detail in Chapter 2, the momentum equation, effectively Newton's $2^{\text {nd }}$ law for a plasma, is

$$
\rho \frac{d \mathbf{v}}{d t}=-\nabla\left(p+\frac{B^{2}}{2 \mu_{0}}\right)+\frac{(\mathbf{B} \cdot \nabla) \mathbf{B}}{\mu_{0}} .
$$

The last term is the tension or curvature force due to bent magnetic field lines. If all the upstream magnetic energy is converted to kinetic energy of the outflowing plasma downstream, then conservation of energy gives

$$
\frac{B_{0}^{2}}{2 \mu_{0}} \sim \frac{1}{2} \rho v_{\text {out }}^{2}
$$

where the left hand side is the energy density stored in the reconnecting magnetic field with strength $B_{0}, \mu_{0}$ is the permeability of free space, and the right hand side is the kinetic energy density of a plasma with density $\rho$ traveling at the outflow speed $v_{\text {out }}$. We note that some magnetic energy is converted to plasma heat, but neglecting this transfer does not alter the conclusions. After solving for $v_{\text {out }}$, one obtains

$$
v_{\text {out }} \sim c_{A} \equiv \frac{B_{0}}{\sqrt{\mu_{0} \rho}}
$$

where $c_{A}$ is the Alfvén speed, the characteristic speed at which perturbations travel along a magnetic field of strength $B_{0}$ in the MHD model.

The rate at which reconnection occurs is related to the convection of the magnetic fields into and out of the dissipation region. This rate can be measured from the electric field $E$ at the X-line which, in the steady state, balances the convection electric field given by Eq. (1.5) 
outside of the dissipation region

$$
E \sim E_{\text {conv }}=v_{\text {in }} B_{0}
$$

From conservation of mass, the mass flux into the dissipation region through the length $L$ must balance the mass flux out the width $\delta$ of the dissipation region

$$
\rho v_{\text {in }} L \sim \rho v_{\text {out }} \delta
$$

where we have assumed equal inflow and outflow densities $\rho_{\text {in }}=\rho_{\text {out }}=\rho$. Using the result for $v_{\text {out }}$ from Eq. (1.8), this gives

$$
v_{\text {in }} \sim c_{A} \frac{\delta}{L}
$$

and the reconnection rate as

$$
E \sim c_{A} B_{0} \frac{\delta}{L}
$$

The ratio $\delta / L$ depends on the mechanism providing dissipation to allow magnetic field lines to change topology.

For the simple case when reconnection results from collisions between the plasma species, we can calculate the reconnection rate; this is called Sweet-Parker reconnection (Parker, 1957; Sweet, 1958). For reconnection to occur at a steady rate, the resistive Ohm's law $\mathbf{E}+\mathbf{v} \times \mathbf{B}=\eta \mathbf{J}$, where $\eta$ is the plasma resistivity implies

$$
E \sim \eta J
$$

where the left side is again the reconnection rate at the $\mathrm{X}$-line, the right side is the collisional 
electric field, and $J$ is the out-of-plane current density. Ampère's Law $\nabla \times \mathbf{B}=\mu_{0} \mathbf{J}$ implies the reconnecting magnetic fields are associated with a current

$$
J \sim \frac{B_{0}}{\mu_{0} \delta}
$$

Using this result in combination with Eqs. (1.9), (1.13), and (1.14) gives

$$
\delta^{2} \sim \frac{\eta L}{\mu_{0} c_{A}} .
$$

Inserting this into Eq. (1.12) gives the resistive reconnection rate $E_{\eta}$

$$
E_{\eta} \sim \sqrt{\frac{\eta c_{A}}{\mu_{0} L} B_{0}^{2}} .
$$

This important result allows us to predict the reconnection rate knowing only upstream properties of the plasma. It reveals that the resistive reconnection rate is much faster than straight diffusion.

\subsubsection{Asymmetric Reconnection}

While much has been learned in studying reconnection between plasmas with equal density and magnetic field strengths on either upstream side of the diffusion region, this theory is nonetheless limited in its applicability. The following presents the generalized theory describing reconnection between a plasma with magnetic field strength $B_{1}$ and plasma density $\rho_{1}$ and another plasma with field strength $B_{2}$ and density $\rho_{2}$, as depicted in Fig. 1.9. The dayside magnetopause is a perfect example of where reconnection is asymmetric, since the magnetosheath can have number densities $n_{\mathrm{SH}} \approx 10-20 \mathrm{~cm}^{-3}$ and magnetic field strengths 


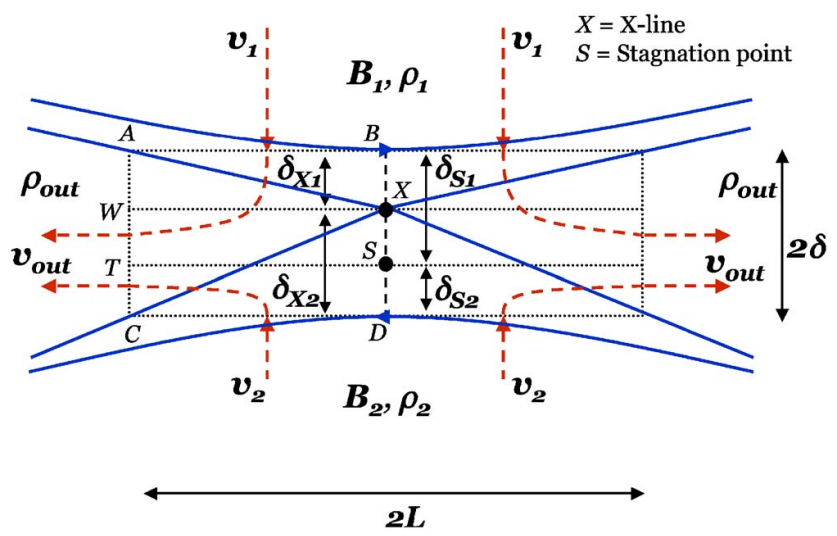

Figure 1.9: Schematic diagram of reconnection occurring between a plasma with density $\rho_{1}$ and magnetic field strength $B_{1}$ and another plasma with density $\rho_{2}$ and magnetic field $B_{2}$. The X-line $(\mathrm{X})$ and stagnation point $(\mathrm{S})$ decouple within the diffusion region, and may be located at two distinct points along the inflow direction. Reprinted from P. A. Cassak and M. A. Shay, Phys. Plasmas, Vol. 14, 102114, 2007. Copyright 2007, with permission from AIP Publishing LLC.

$B_{\mathrm{SH}} \approx 10-20 \mathrm{nT}$ as compared to those of the magnetosphere $n_{\mathrm{MS}} \sim 0.1 \mathrm{~cm}^{-3}$ and $B_{\mathrm{MS}} \approx 50-$ $60 \mathrm{nT}$ [see Malakit et al. (2010), and references therein].

Asymmetries in the upstream plasma densities and reconnecting magnetic field strengths have important consequences on how mass and magnetic flux enter the dissipation region during steady state reconnection. Conservation of magnetic flux requires the reconnection rate $E$ at the $\mathrm{X}$-line to balance the convective electric field on either upstream side of the dissipation region; this is given by

$$
E \sim v_{1} B_{1} \sim v_{2} B_{2}
$$

where $v_{1}$ and $v_{2}$ are the inflow speeds on either side of the dissipation region as depicted in Fig. 1.9, implying that the convection of the upstream magnetic fields into the dissipation region occurs at different rates. The mass flux into the dissipation region is also conserved, and the conservation of these two fluxes results in important changes to the dissipation region's internal structure, where the X-line and stagnation point are no longer at the dissipation 
region's center and can occupy different locations along the inflow direction in asymmetric systems (Cassak and Shay, 2007). Conservation of magnetic flux causes the X-line to be displaced towards the weaker magnetic field's side given by the magnetic field ratio $b$

$$
\frac{\delta_{X 1}}{\delta_{X 2}} \sim \frac{B_{1}}{B_{2}} \equiv b,
$$

where $\delta_{X 1}$ and $\delta_{X 2}$ are the distances from the X-line to the dissipation region's upstream edges that border each plasma, 1 or 2. Similarly, conservation of mass and magnetic flux into the dissipation region offsets the stagnation point from each edge, given by

$$
\frac{\delta_{S 1}}{\delta_{S 2}} \sim \frac{\rho_{1} B_{2}}{\rho_{2} B_{1}},
$$

where $\delta_{S 1}$ and $\delta_{S 2}$ are the distances measured from the upstream edge of the dissipation region to the stagnation point.

Modifications to Eqs. (1.8) and (1.12) can be obtained using the conservation of mass, energy, and magnetic flux. The result is that the outflow plasma mass density $\rho_{\text {out }}$ scales as (Cassak and Shay, 2007)

$$
\rho_{\text {out }} \sim \frac{\rho_{1} B_{2}+\rho_{2} B_{1}}{B_{1}+B_{2}},
$$

which is the average plasma mass density weighted by the strengths of the reconnecting magnetic fields. The outflow speed can be shown to be

$$
c_{A, \text { asym }}^{2} \sim \frac{B_{1} B_{2}}{\mu_{0} \rho_{\text {out }}},
$$

which is a hybrid Alfvén speed based on the geometric mean of the magnetic field strengths 
$\sqrt{B_{1} B_{2}}$ and outflow density. The asymmetric reconnection rate can be calculated as

$$
E_{\text {asym }} \sim \frac{B_{1} B_{2}}{B_{1}+B_{2}} c_{A, a s y m} \frac{2 \delta}{L} .
$$

One can generalize the expression in Eq. (1.16) for asymmetric Sweet-Parker reconnection. Proceeding as before where the upstream convective electric field scales similarly to the resistive electric field at the $\mathrm{X}$-line, one can arrive at an expression for the asymmetric Sweet-Parker reconnection rate (Cassak and Shay, 2007)

$$
E_{\eta, \text { asym }} \sim \sqrt{\frac{\eta c_{A, \text { asym }}}{\mu_{0} L} B_{1} B_{2}}
$$

which is proportional to the geometric mean of the upstream magnetic field strengths.

In concluding this section, we note that under symmetric conditions, i.e., $B_{1}=B_{2}=B_{0}$ and $\rho_{1}=\rho_{2}=\rho$, Eqs. (1.18) and (1.19) equal 1, indicating the X-line and stagnation point are co-located at the center of the diffusion region, Eq. (1.20) reduces to $\rho$, and that

Eqs. (1.21), (1.22), and (1.23) reduce to Eqs. (1.8), (1.12), and (1.16), respectively, as they must. The reader is referred to Cassak and Shay (2007) for a more thorough derivation of these expressions.

\subsubsection{The Effects of Sheared Plasma Flows on Magnetic Recon- nection}

The discussion up to this point assumed that the upstream plasmas have no flows parallel to the reconnecting magnetic field. This need not be the case, as commonly seen at the magnetospheric cusps for northward IMF conditions, where the magnetosheath plasma flows 


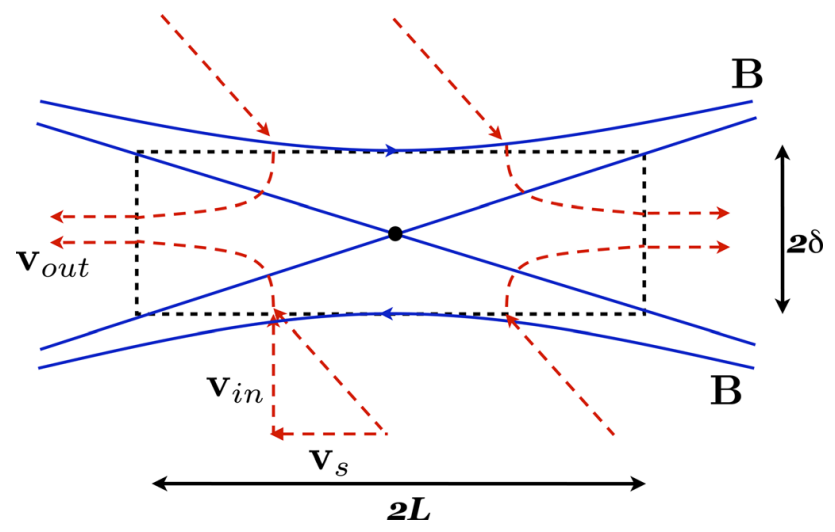

Figure 1.10: Schematic diagram of reconnection between oppositely directed magnetic fields of strength $B$ with a flow component $v_{s}$ parallel to the magnetic field. See caption of Fig. 1.8 for definitions. Reprinted from P. A. Cassak, Phys. Plasmas, Vol. 18, 072106, 2011. Copyright 2011, with permission from AIP Publishing LLC.

around the magnetopause with sizable velocity components parallel to the magnetosheath magnetic field [see Wilder et al. (2014), and references therein].

Figure 1.10 displays the dissipation region (dashed black line) with an upstream sheared flow component $v_{s}$ parallel to the reconnecting magnetic field undergoing symmetric reconnection. It has long been known that a velocity shear parallel to the reconnecting magnetic field impacts the efficiency of reconnection (Chen and Morrison, 1990; La Belle-Hamer et al., 1994, 1995; Cassak and Otto, 2011). In fact, if this sheared flow is large enough $v_{s}>c_{A}$, reconnection does not occur, and the Kelvin-Helmholtz instability can occur. Cassak (2011) presented the theoretical framework for symmetric reconnection systems with a sheared flow speed and showed the reconnection rate in such systems scales as

$$
E_{\text {shear }} \sim E\left[1-\left(\frac{v_{s}}{c_{A}}\right)^{2}\right]
$$

where $E$ and $c_{A}$ are given by Eqs. (1.12) and (1.8), respectively.

Figure 1.11 displays the dissipation region of an asymmetric reconnection system with 


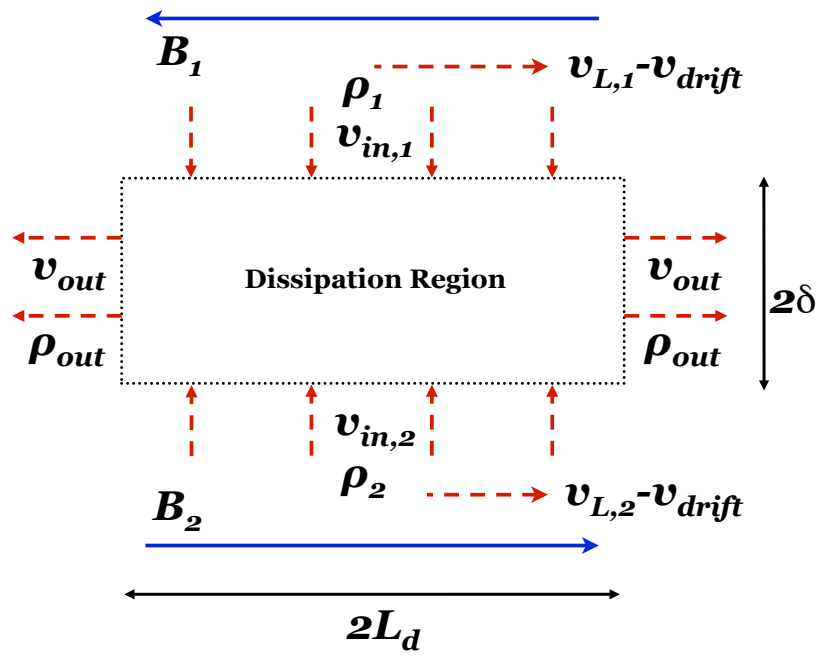

Figure 1.11: Schematic diagram of reconnection occurring between plasmas with different plasma densities and magnetic field strengths. See caption of Fig. 1.9 for definitions. Reprinted with permission from C. E. Doss, in preparation to be submitted to J. Geophys. Res.

flows parallel to the reconnecting magnetic fields; we note the features internal to the dissipation region are similar to those depicted in Fig. 1.9, but are not shown. It was initially thought that the results of Cassak (2011) could easily be extended to asymmetric reconnection systems by replacing $E_{0}$ and $c_{A}$ in Eq. (1.24) by their asymmetric counterparts and replacing $v_{s}$ with the asymmetric shear flow speed $v_{\text {shear }}$ defined as

$$
v_{\text {shear }} \equiv \frac{1}{2}\left(v_{L, 1}-v_{L, 2}\right)
$$

where $v_{L, 1}$ and $v_{L, 2}$ are the magnitudes of the upstream parallel velocity components; this model is discussed more thoroughly in Chapter 4.3.4. However, it was recently shown that this is not correct and the asymmetries have important consequences on reconnection when a flow shear is present (Doss et al., in prep.). The first is that the X-line drifts at a speed $v_{d r i f t}$ resulting from the conservation of the upstream parallel momentum carried through to the X-line. In the reference frame of the drifting $\mathrm{X}$-line and using the conservation of the 
parallel momentum and magnetic flux, one can show the drift speed $v_{d r i f t}$ is

$$
v_{d r i f t} \sim \frac{\rho_{1} B_{2} v_{L 1}+\rho_{2} B_{1} v_{L, 2}}{\rho_{1} B_{2}+\rho_{2} B_{1}}
$$

where $\rho_{1}, \rho_{2}, B_{1}$ and $B_{2}$ are the upstream asymmetric plasma parameters as before. This result was shown to agree with observations of reconnection at the magnetospheric cusps, where Cluster spacecraft measured an X-line traveling tailward in the outflow direction (Wilder et al., 2014).

A second result is that the reconnection rate is again reduced in this system, where reconnection is slowed due to the parallel flow opposing the tension force of the newly reconnected field line's tension at the X-line. The reconnection rate for this system can be shown to scale as

$$
E_{\text {shear }, \text { asym }} \sim E_{\text {asym }}\left[1-\frac{v_{\text {shear }}^{2}}{c_{A, \text { asym }}^{2}} \frac{4 \rho_{1} B_{2} \rho_{2} B_{1}}{\left(\rho_{1} B_{2}+\rho_{2} B_{1}\right)^{2}}\right]
$$

where $E_{\text {asym }}$ is given by Eq. (1.22). We note that in the symmetric reconnection limit, Eq. (1.27) reduces to Eq. (1.24). As before, the Kelvin-Helmholtz instability dominates at sufficiently high parallel sheared flow speeds resulting in reconnection's suppression.

\subsection{The Global Picture of Magnetospheric Reconnec- tion}

Section 1.4 discussed the physics local to a reconnection site. However, the magnetospheric convection process discussed in Section 1.2 shows that reconnection is a global phenomena. 
Here, we discuss global properties of magnetic reconnection.

Dungey's simplified magnetospheric reconnection model correctly identified reconnection for cases when there is no tilt to Earth's magnetic dipole and when the IMF is perfectly directed either northward or southward (Dungey, 1961). This model correctly predicted the magnetospheric convection resulting from reconnection. However, this model is limited in its scope as Earth's magnetic dipole makes an approximate angle of $11^{\circ}$ with its rotation axis, and, additionally, the IMF can have an arbitrary orientation with the geomagnetic dipole. In fact, it is not presently possible to quantitatively predict the location of magnetic reconnection at Earth's dayside magnetopause and the resulting magnetospheric convection for arbitrary IMF and dipole tilt. It is therefore necessary to go beyond the sketches of Dungey (1961, 1963).

We first consider the case of southward IMF as depicted in Fig. 1.4(a). This plot only shows two points where reconnection happens. However, the magnetosphere is threedimensional. In the previous section, we discussed reconnection occurring in a $2 \mathrm{D}$ plane, where we defined the $\mathrm{X}$-line as the location where reconnection occurs. One could picture "stacking" the 2D reconnection planes in the out-of-plane direction to get a representation

of how reconnection could happen in $3 \mathrm{D}$. The points where reconnection occurs now form a line. In 3D, this line need not be straight. For dayside reconnection, the line along which reconnection occurs is a curve that lies at the dayside magnetopause. This curve is called the magnetic separator, which we discuss in the next section.

\subsubsection{Magnetic Separators}

The work presented in this dissertation takes several steps in going beyond Dungey's model by identifying locations that separate magnetic fields of different topologies, as this is where 

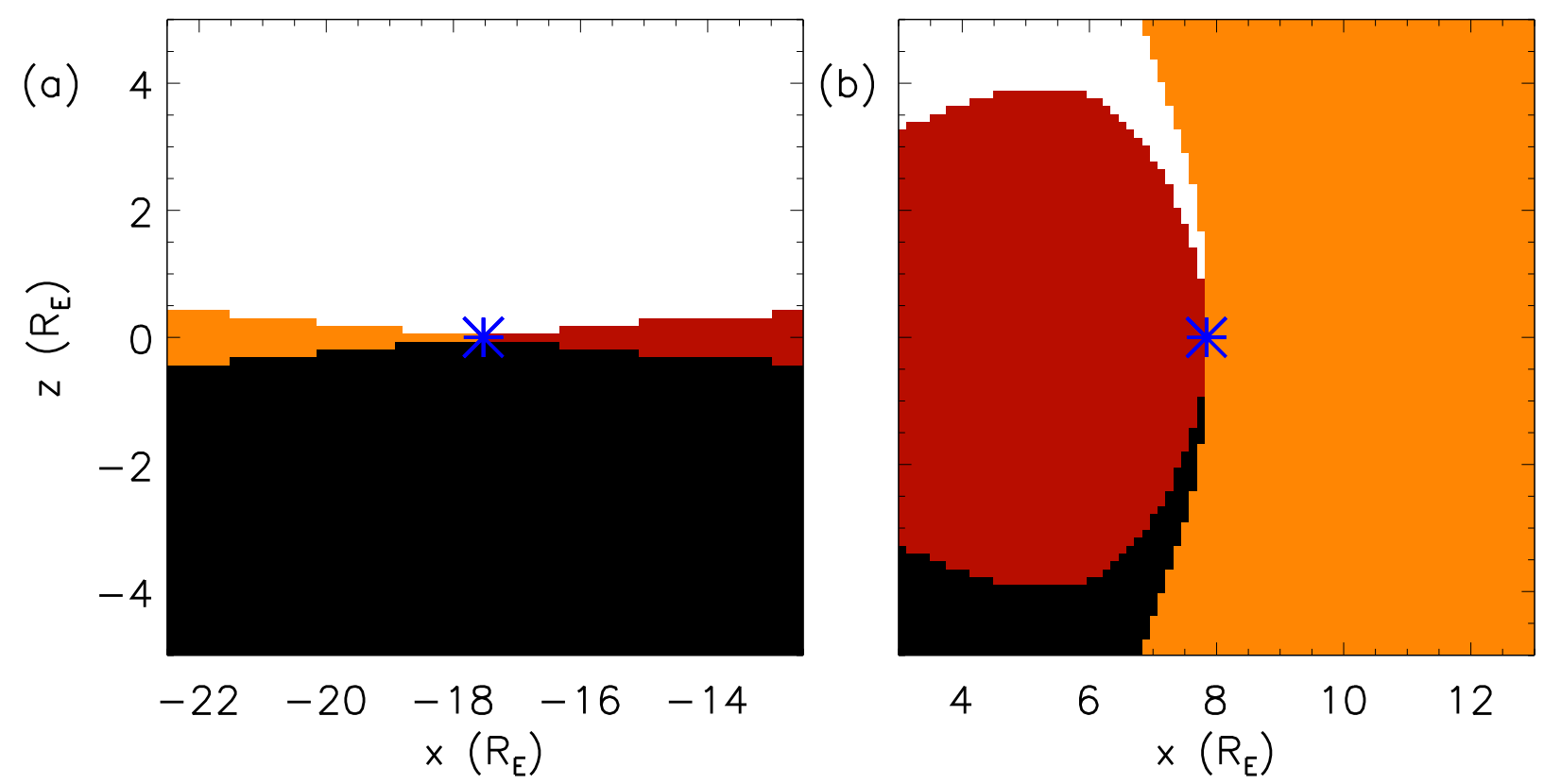

Figure 1.12: The intersection of flux surfaces with planes centered at (a) $(-17.5,0,0)$ and (b) $(8,0,0) R_{E}$ (GSM) for the subsolar magnetopause and magnetotail, respectively, in a simulation with southward IMF and no dipole tilt. Closed geomagnetic flux is colored red, solar wind magnetic flux is colored orange, and open magnetic flux connecting to the south and north geomagnetic poles are colored black and white, respectively. The approximate intersection of all four flux surfaces is the magnetic separator, and is marked with blue asterisks $(*)$.

reconnection occurs. To motivate this, we turn our focus back to the magnetosphere under southward IMF conditions as depicted in Fig. 1.4(a). In this figure, several magnetic field lines have been traced and are color coded according to where each maps relative to Earth. For example, the closed geomagnetic field lines map to Earth's northern and southern geomagnetic pole and are color-coded red; likewise, the IMF does not map to either geomagnetic pole and are colored yellow. Once the IMF and geomagnetic field enter the magnetopause and undergo magnetic reconnection, two new "open" magnetic topologies are created that map to one of Earth's poles at one side and out to the solar wind in the other direction; these two magnetic topologies are color coded gray in Fig. 1.4(b).

These magnetic field lines occupy large volumes of space where one can define a flux surface or separator surface, which separates regions of differing magnetic topology. Fig- 
ure 1.12 shows slices through the reconnection sites (a) in the magnetotail and (b) at the subsolar magnetopause in the southward IMF simulation originally depicted in Fig. 1.4(a). Several magnetic field lines are traced in planes centered at (a) $\mathbf{r}=(-17.5,0,0)$ and (b) $\mathbf{r}=(8,0,0) \mathrm{R}_{\mathrm{E}}(\mathrm{GSM})$, with magnetic field lines traced at points separated by $0.125 \mathrm{R}_{\mathrm{E}}$ along the $x$ and $z$ axes; the technique used to determine a magnetic field line's topology is presented in Chapter 3.1.1. In each of these slices, closed geomagnetic field lines are color coded red, orange for solar wind magnetic fields, and black or white corresponding to open field lines connecting to either the southern or northern geomagnetic poles, respectively. All four flux surfaces intersect at (a) $\mathbf{r}_{S e p}=(-17.53,0,0)$ and (b) $\mathbf{r}_{S e p}=(7.84,0,0) \mathrm{R}_{\mathrm{E}}$ and each intersection is displayed with a blue asterisk; this intersection corresponds to the magnetic separator, which lies at the intersection of all four magnetic topologies.

The separator depicted in Fig. 1.12 marks the locations of reconnection at the subsolar magnetopause and in the magnetotail, in agreement with Dungey (1961). As we motivated at the beginning of this section, the interplanetary and geomagnetic fields still point northward and southward in other planes along the $y$-axis. The magnetic fields are oppositely directed in each of these planes so reconnection is possible. In practice, we find many other locations along the dayside magnetopause where the four magnetic topologies meet, with the separator extending in three-dimensions. We note that the separator is the three-dimensional (3D) analog to the X-line depicted in Figs. 1.8 and 1.9.

As the separator is the boundary of different magnetic topologies, it therefore marks individual locations where reconnection can occur between geomagnetic and solar wind magnetic field lines at the dayside magnetopause, and likewise between the two open magnetic fluxes in the distant magnetotail. Local to the separator, some component of the magnetic field is oppositely directed, and it reconnects at the separator when it enters the diffusion region and 


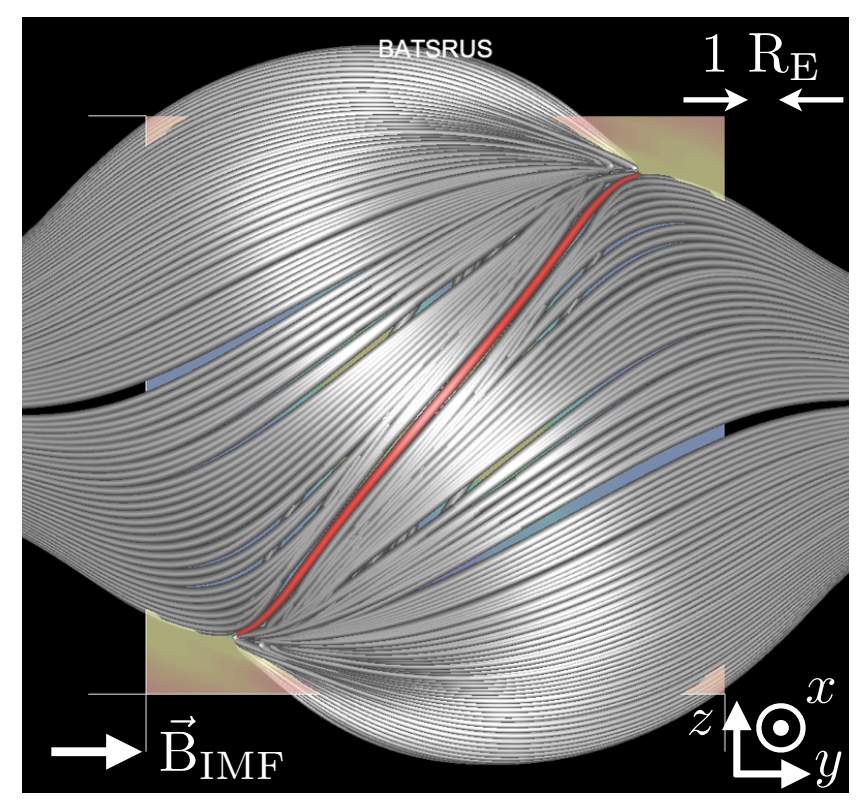

Figure 1.13: The dayside separator (red) in a global magnetospheric simulation where the IMF has clock angle $\theta_{\mathrm{IMF}}=90^{\circ}$. Northern- and southern-open magnetic field lines are located on the left and right, respectively. Outside and inside of the depicted surface are solar wind and closed geomagnetic field lines, respectively.

breaks at the separator. Similar to the 2D cases discussed in Sections 1.4.1 and 1.4.2, reconnection sets up an electric field perpendicular to the reconnecting magnetic fields, which is parallel to the separator for 3D. Using the electric field along the separator, one can calculate a potential along the entire separator which gives the global reconnection rate $\Phi_{\text {Sep }}$ along the separator. This can be calculated with the integral

$$
\Phi_{S e p}=\oint \mathbf{E} \cdot d \mathbf{L}_{S e p}=\oint[-(\mathbf{v} \times \mathbf{B})+\eta \mathbf{J}] \cdot d \mathbf{L}_{S e p}
$$

along the separator's length $\mathbf{L}_{\text {Sep }}$ using the electric field $\mathbf{E}$ given by the resistive Ohm's law (Schindler et al., 1988). This separator potential can then be compared with the cross polar cap potential difference $\Delta \Phi_{P C}$ given by Eq. (1.4), which is often calculated as a proxy for the global rate of reconnection at the separator (Siscoe et al., 2001; Lopez et al., 2010).

Separators can be defined for arbitrary IMF orientations and dipole tilts at the magne- 
topause (Cowley, 1973; Siscoe, 1987; Siscoe et al., 2001). Take for example the magnetopause shown in Fig. 1.13, which depicts the magnetic separator in a numerical simulation described in Chapter 2 without a dipole tilt and where the IMF has a clock angle of $90^{\circ}$. The separator is displayed as the red field line and lies between the gray northern- and southern-open field lines on the left and right, respectively. Inside of the depicted surface are closed terrestrial magnetic field lines whereas the interplanetary magnetic fields lie immediately outside. This figure demonstrates that a magnetic separator can be determined for IMF oriented obliquely with Earth's dipole axis; an accomplishment of this dissertation work is the development of a robust technique to find separators in global magnetospheric simulations.

\subsubsection{Observations of Separators at the Dayside Magnetopause}

Separators are notoriously difficult to measure in situ at the dayside magnetopause with single spacecraft. However, Pu et al. (2013) used electron pitch angle measurements onboard the Chinese Double Star satellite to infer magnetic topologies at a coiled magnetic field line flanked by two active X-lines on the dayside magnetopause; this circular magnetic flux tube is called a Flux Transfer Event (FTE) (Russell and Elphic, 1978). They observed the standard four magnetic topologies described previously, albeit the topologies were coiled due to reconnection occurring continuously at both X-lines.

\subsubsection{Observations of Separators in the Magnetotail}

Xiao et al. (2007) found a pair of magnetic nulls connected by the separator with length $\sim 1,000 \mathrm{~km}$ in the magnetotail using the four Cluster spacecraft as the satellites passed through the magnetotail on October 1, 2001. This separator line was located near the ecliptic plane in the nightside-dawn sector at $(-16.2,7.9,0.5) \mathrm{R}_{E}$ in GSM coordinates. Work 
presented by Guo et al. (2013) fed the observed magnetic fields from Cluster to a magnetotail magnetic field model in order to extrapolate the magnetic field in three dimensions to better investigate the physics local to the separator observed in Xiao et al. (2007). Two regimes of magnetic reconnection were measured: the first measured reconnection between nearly anti-parallel magnetic fields, whereas the second measured reconnection with a significant magnetic field component parallel to the separator. Guo et al. (2013) note that both of these reconnection regimes were topologically similar, albeit the measured magnetic field parallel to the separator differed by an order of magnitude.

In concluding this section, these observations of separators motivate that separators are important for reconnection occurring at the magnetopause and in the magnetotail. However, given their difficulty to measure observationally, we use global magnetospheric simulations where it is much easier to identify separators in order to understand the role reconnection plays in solar wind-magnetosphere coupling.

\subsection{Dayside Reconnection as Either a Global or Local Process}

There has been increased debate over a longstanding question about whether magnetospheric reconnection is a local or global phenomenon. By a global process, it is meant that reconnection is entirely determined by the boundary conditions imposed by the solar wind. For example, Newell et al. (2007) reviews several different empirical models which only use the solar wind plasma parameters $\left(v_{\mathrm{SW}}, B_{\mathrm{SW}}, n_{\mathrm{SW}}\right.$, etc. $)$ to predict the solar wind's "geoeffectiveness," that is, the coupling efficiency of the solar wind with Earth's magnetosphere. In this interpretation, the physics at the dayside reconnection site adjusts in response to the lo- 

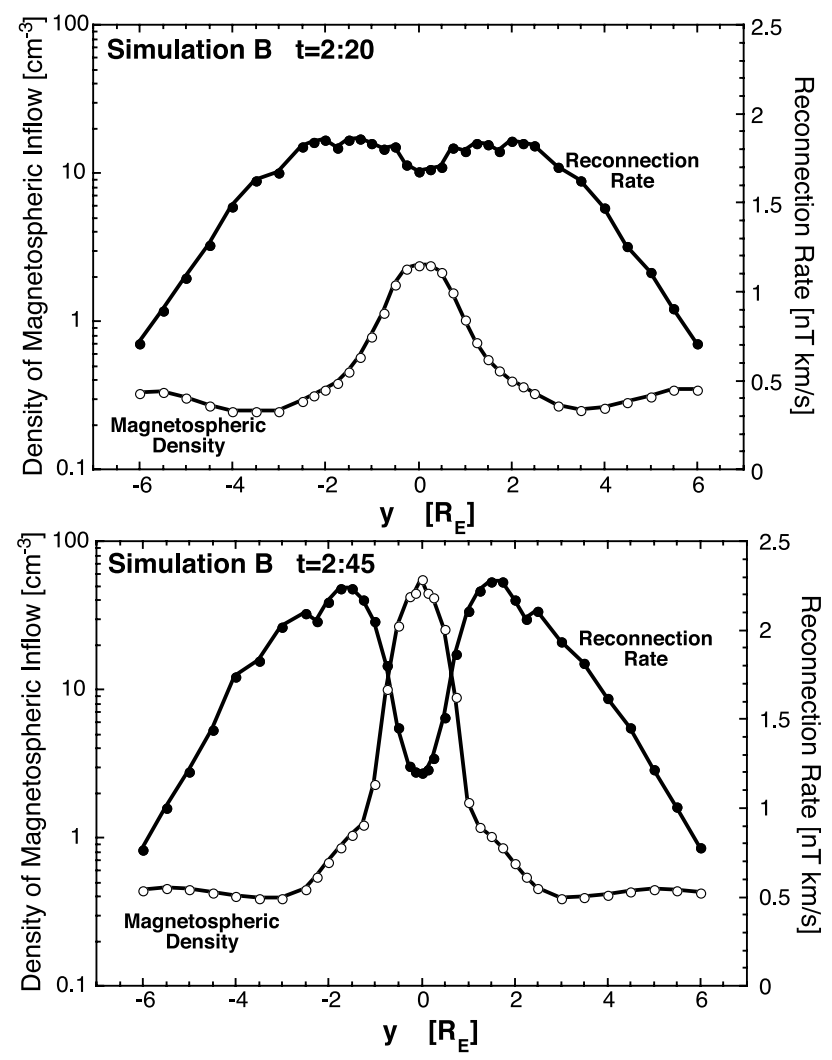

Figure 1.14: Results from a simulation of the dayside magnetopause with southward IMF (top) before and (bottom) after dense plasmaspheric plasma encounters the reconnection site. The measured reconnection rate $\eta J_{y}(\bullet)$ and magnetospheric density $(\circ)$ is displayed for both these times. Reprinted from J. Borovsky et al., J. Geophys. Res., Vol. 113, A07210, 2008. Copyright 2008, with permission from John Wiley and Sons.

cal solar wind plasma. Recent arguments, however, suggest the control can be local, meaning that the plasma conditions of the magnetosphere local to the reconnection site have an important role in controlling reconnection at the magnetopause. For example, magnetospheric observations (Borovsky and Denton, 2006; Walsh et al., 2014) and simulations (Borovsky et al., 2013b) present evidence of plasmaspheric drainage plumes encountering the dayside magnetopause with measurable decreases in reconnection.

Figure 1.14 displays results from a simulation of the dayside magnetopause with southward IMF at times (top) before and (bottom) after the plasmasphere increases the magnetospheric density at the reconnection site. In this figure, the reconnection rate $\eta J_{y}$ at the 
magnetopause is displayed with solid black circles and the magnetospheric density just inside the magnetopause is displayed with open circles. Before the magnetospheric plasma is enhanced, the reconnection rate at the subsolar magnetopause $\left(|y| \leq 3 \mathrm{R}_{\mathrm{E}}\right)$ is constant around $2 \mathrm{nT} \mathrm{km} / \mathrm{s}$ and the magnetospheric density $n \lesssim 1 \mathrm{~cm}^{-3}$. Once the plasmasphere populates the magnetosphere $\left(n \approx 50 \mathrm{~cm}^{-3}\right)$, the reconnection rate at the subsolar point $\left(y \approx 0 \mathrm{R}_{\mathrm{E}}\right)$ falls to $1.2 \mathrm{nT} \mathrm{km} / \mathrm{s}$. These results have been used as evidence that local physics can control the rate of reconnection.

However, the debate is far from settled. The Borovsky et al. (2008) simulations show the local reconnection rate is changed by the plume. However, it was shown that the integrated global reconnection potential calculated from Eq. (1.28) over the whole separator is essentially unchanged (to within $3 \%$ ) before and after the plume hits (R. Lopez, private communication, 2014); the reconnection potential is equal to the area under the solid black curves depicted in Fig. 1.14. This occurs because the local reconnection rate just outside where the plume hits $\left(y \approx \pm 2 \mathrm{R}_{\mathrm{E}}\right)$ increases to $2.25 \mathrm{nT} \mathrm{km} / \mathrm{s}$. In explaining this, the claim is made that although reconnection is locally hindered at the subsolar magnetopause due to the increased magnetospheric mass density, the solar wind magnetic flux flows around the magnetopause to sectors adjacent to the density increase where reconnection is enhanced, and thus conserving the global reconnection rate.

We note that we do not solve this problem here, but it is a motivating factor in developing techniques to measure local and global properties of reconnection in global magnetospheric simulations. 


\subsection{Statement of the Problem}

Given the importance of reconnection to the global configuration and dynamics of Earth's magnetosphere, it is important to identify magnetic separators in Earth's magnetosphere as possible locations of magnetic reconnection. The magnetic separator is a global structure existing in Earth's magnetosphere and, although satellite observations have identified separators, very little can be inferred about the global reconnection process from these point measurements.

Studying reconnection in global simulations with arbitrary solar wind conditions is challenging because it is difficult to locate where reconnection happens. It would be much easier to analyze reconnection - for example, to address questions of local versus global control if the separator could be found, although identifying magnetic separators in global magnetospheric simulations is non-trivial. Several studies in the literature presented techniques to identify separators in global magnetospheric simulations (Laitinen et al., 2006; Dorelli et al., 2007; Hu et al., 2009; Ouellette et al., 2010). However, these techniques have a few disadvantages: (1) large numbers of field lines need to be traced throughout the simulation domain in order to determine where the four magnetic topologies converge, thus decreasing their efficiency, (2) these techniques rely on magnetospheric symmetry in cases without any dipole tilt to find the separator's location on the sun-Earth line, and (3) some do not work

for northward IMF conditions. We develop a general approach to locate separators in global simulations.

A number of models have been proposed to predict where magnetic reconnection occurs on the dayside magnetopause (Sonnerup, 1974; Gonzalez and Mozer, 1974; Alexeev et al., 1998; Moore et al., 2002; Trattner et al., 2007; Swisdak and Drake, 2007; Borovsky, 2008, 
2013; Hesse et al., 2013); these models will be reviewed in Chapter 4. However, there has been no careful assessment of the correctness of these models in global magnetospheric simulations because it has been prohibitively difficult to find where reconnection occurs. With our new technique to find separators, we perform a systematic test of these models.

Finally, there are studies that have analyzed the reconnection locally at the magnetic separator (Borovsky et al., 2013b; Ouellette et al., 2014). However, many of these previous studies focused primarily on cases where reconnection is confined to the ecliptic plane occurring when the IMF is due southward and with no magnetospheric dipole tilt. While studies have identified magnetic separators in simulations with or without a dipole tilt and IMF oriented obliquely to the dipole, none have analyzed the reconnection dynamics local to the separator (Laitinen et al., 2006; Dorelli et al., 2007; Hu et al., 2009; Ouellette et al., 2010; Hoilijoki et al., 2014). Instead, these studies have largely focused on identifying magnetic separators and emphasized the global implications of separator reconnection throughout the magnetosphere. We use global simulations to study reconnection in the magnetosphere locally, to begin to make contact with the local versus global question.

\subsection{Summary of Results}

In this dissertation, we identify separators arising in global magnetospheric resistive magnetohydrodynamic simulations in order to analyze reconnection in Earth's magnetosphere. Chapter 2 describes the resistive magnetohydrodynamic (MHD) model of plasmas and the setup of our high-resolution global magnetospheric simulations.

In Chapter 3, we present a simple, efficient and accurate algorithm to map magnetic separators in global magnetospheric simulations at the dayside magnetopause for arbitrary 
IMF clock angle (Komar et al., 2013). We verify the technique using exact solutions for an analytic model involving the superposition of uniform and dipolar magnetic fields. Then, we trace separators in global resistive magnetohydrodynamic (MHD) simulations for various IMF clock angles, and show that the last closed field line, an often used proxy for the dayside separator, does not map the separators for southward IMF. We discuss trends in magnetic null locations and magnetic separators, making comparisons to the analytical field model. Results shown in Chapters 4 and 5 also show that this technique works for different solar wind conditions and in cases with a dipole tilt.

Chapter 4 presents a systematic test of the predictions of several models of where dayside reconnection occurs and compares these predictions with the separators arising in selfconsistent global simulations of the magnetosphere (Komar et al., 2015). We employ image processing techniques to unambiguously determine each model's prediction in our simulations. Each model's prediction is then compared with the magnetic separator and we test each model's agreement with the separators for various IMF clock angles $\theta_{\text {IMF }}$, IMF strengths $B_{\mathrm{IMF}}$, and a case with dipole tilt angle. We also present a model of one possible way to incorporate the effects of a flow shear parallel to the reconnecting fields into the models based on reconnection outflow speed and reconnection rate, although recent results suggest this model is likely incorrect (Doss et al., in prep.). In general, we find the maximum magnetic shear angle model (Trattner et al., 2007) does best in predicting the separator for northward IMF conditions, and that a number of models do equally well in determining the dayside separator for southward IMF conditions.

Finally, we carefully investigate how reconnection is distributed along the separator in Chapter 5. We do this by taking planes perpendicular to the separator to measure the upstream plasma parameters local to the separator. Using these measured parameters we 
calculate the generalized and Sweet-Parker reconnection rates given by Eqs. (1.22) and (1.23) and compare these values with the measured reconnection rates in these planes to assess the applicability of local asymmetric reconnection theory. We find the measured reconnection parameters scale decently well with their theoretical counterparts along the dayside separator in simulations with a southward IMF orientation $\left(\theta_{\mathrm{IMF}} \geq 90^{\circ}\right)$ and IMF strength of $20 \mathrm{nT}$. The scaling relations agree well in a simulation with $\theta_{\mathrm{IMF}}=120^{\circ}$ and a dipole tilt. However, the measured reconnection rates scale less well when the IMF strength is lowered to 5 and $2 \mathrm{nT}$ in simulations with IMF clock angle of $120^{\circ}$; we argue that this results from numerical effects in our simulations. 


\section{Chapter 2}

\section{Magnetospheric Simulation Study}

\subsection{The Governing Equations of Resistive Magnetohy- drodynamics}

Magnetohydrodynamic (MHD) theory is a simplified description of plasma phenomena which treats the ions and electrons as a single fluid while incorporating electromagnetic forces given by Maxwell's equations. In collisional plasmas, the electric field $\mathbf{E}$ is given by the resistive Ohm's law

$$
\mathbf{E}+\mathbf{u} \times \mathbf{B}=\eta \mathbf{J}
$$

where $\mathbf{u}$ is the plasma bulk velocity, $\mathbf{B}$ is the magnetic field, $\eta$ is the plasma resistivity due to electron-ion collisions, and $\mathbf{J}$ is the current density. The $\mathbf{u} \times \mathbf{B}$ term is the convective electric field and $\eta \mathbf{J}$ is the resistive electric field due to collisions analogous to the field in a wire.

The other equations of MHD are presented in conservative form below, describing the temporal and spatial evolution of a plasma with mass density $\rho$ and thermal pressure $p$, 
assumed scalar for simplicity. The continuity equation

$$
\frac{\partial \rho}{\partial t}+\nabla \cdot(\rho \mathbf{u})=0
$$

states that total mass is conserved and implies that for a plasma element's mass density to change, mass flux must enter or leave the plasma element. Similarly, a plasma element's momentum $\rho \mathbf{u}$ will change if momentum flux enters or leaves the element's boundary, and is described by the momentum equation

$$
\frac{\partial(\rho \mathbf{u})}{\partial t}+\nabla \cdot\left[\rho \mathbf{u u}+\left(p+\frac{B^{2}}{2 \mu_{0}}\right) \mathbf{I}-\frac{\mathbf{B B}}{\mu_{0}}\right]=0
$$

where $\mu_{0}$ is the permeability of free space and $\mathbf{I}$ is the unit tensor. The terms inside the brackets describe transport of momentum flux due to the plasma bulk flow [ $\rho \mathbf{u u}]$, from total pressure (which is the sum of the thermal and magnetic pressures) $\left[p+B^{2} /\left(2 \mu_{0}\right)\right]$, and from magnetic curvature $\left[\mathbf{B B} / \mu_{0}\right]$. Lastly, the total energy $\mathcal{E}$ of the plasma element can only change if energy flux flows into or out of the element's boundary and is given by the energy equation,

$$
\frac{\partial \mathcal{E}}{\partial t}+\nabla \cdot\left[(\mathcal{E}+p) \mathbf{u}-\frac{(\mathbf{u} \cdot \mathbf{B})}{\mu_{0}} \mathbf{B}+\frac{\eta}{\mu_{0}} \mathbf{J} \times \mathbf{B}\right]=0
$$

where $\gamma$ is the ratio of specific heats $(\gamma=5 / 3$ for our purposes $), \mathcal{E} \equiv \rho u^{2} / 2+p /(\gamma-1)+$ $B^{2} /\left(2 \mu_{0}\right)$ is the total energy density where the first term is the kinetic energy density, the second is the internal energy density, and the third is the magnetic energy density. The terms inside the brackets correspond to the transport of energy flux, and by breaking $\mathcal{E}$ into its constituent terms gives the kinetic energy flux $\left[\left(\rho u^{2} / 2\right) \mathbf{u}\right]$, heat enthalpy $[\gamma p /(\gamma-1) \mathbf{u}]$, the electromagnetic energy entering or leaving the fluid element $\left[B^{2} /\left(2 \mu_{0}\right) \mathbf{u}-(\mathbf{u} \cdot \mathbf{B}) \mathbf{B} / \mu_{0}\right]$, 
which is related to the Poynting flux $\mathbf{E} \times \mathbf{B} / \mu_{0}$, and Ohmic heating $\left[\eta(\mathbf{J} \times \mathbf{B}) / \mu_{0}\right]$.

MHD is a useful model for the global magnetosphere as it is good at describing nonrelativistic phenomena $(|\mathbf{u}| \ll c)$ for time scales larger than the ion gyrofrequency $\Omega_{i}^{-1} \equiv$ $\left(e B / m_{i}\right)^{-1}$ and at spatial scales larger than the Larmor radius $r_{L} \equiv|\mathbf{u}| / \Omega_{i}$. Maxwell's equations simplify with these assumptions and, for resistive MHD, Faraday's law becomes

$$
\frac{\partial \mathbf{B}}{\partial t}=-\nabla \times \mathbf{E}=\nabla \times(\mathbf{u} \times \mathbf{B}-\eta \mathbf{J}),
$$

where we have substituted the electric field given by Eq. (2.1). The first term on the right hand side of Eq. (2.5) contains the physics describing magnetic fields moving with the plasma flow and resulting in the convection, bending, or compression of the magnetic field. The second term is the magnetic diffusion term which acts to smooth out magnetic field gradients by converting magnetic energy into thermal energy. Ampère's law relates the current density to the magnetic field

$$
\nabla \times \mathbf{B}=\mu_{0} \mathbf{J}
$$

and the magnetic Gauss' Law gives

$$
\nabla \cdot \mathbf{B}=0
$$

We note that one can obtain the ideal MHD equations by setting $\eta=0$ (Alfvén, 1942).

\subsection{The Code and its Initialization}

We perform global simulations using the Space Weather Modeling Framework (SWMF) (Tóth et al., 2005; Tóth et al., 2012), a suite of physical models developed at the University of Michigan and used to model regions from the sun to the magnetosphere and beyond, although 
the methods detailed in this dissertation can be adapted to other global magnetospheric codes. We specifically employ the Block Adaptive Tree Solarwind Roe-type Upwind Scheme (BATS-R-US) MHD code to solve the resistive MHD equations on a high resolution, threedimensional, rectangular, irregular grid in order to simulate the global magnetosphere (Powell et al., 1999; Gombosi et al., 2000; De Zeeuw et al., 2000). The ionosphere is modeled with the ionospheric electrodynamics (IE) component. A brief synopsis of the BATS-R-US MHD code is discussed in Appendix B.

The simulations are performed at NASA's Community Coordinated Modeling Center (CCMC), a freely available code repository administered by NASA Goddard Space Flight Center. The CCMC's Kameleon software suite, which was developed by the CCMC to analyze the standardized output of different simulation models performed at the CCMC, is used to partially analyze simulation output of BATS-R-US in order to sample data and trace magnetic field lines at arbitrary coordinates within the simulation domain. The simulation domain is $-255<x<33,-48<y<48$, and $-48<z<48$, where distances are measured in $\mathrm{R}_{\mathrm{E}}$ and the coordinate system is GSM. The simulations are run using BATS-R-US version 8.01. The simulations use constant solar wind values for its boundary condition at $x=33 \mathrm{R}_{\mathrm{E}}$, although BATS-R-US is capable of using event data measured by solar wind monitors. The simulations are evolved for two hours (02:00:00) of magnetospheric time and we look at the 02:00:00 mark of simulation data when the dayside magnetopause has achieved a quasisteady state. This was determined by comparing the location of the current density $J_{y}$ along the $x$-axis at adjacent time outputs (every 00:10:00); we find the current layer along the $x$-axis is approximately stationary after 01:30:00 of magnetospheric time. The standard high-resolution grid for CCMC simulations has 1,958,688 grid cells with a coarse resolution of $8 R_{E}$ in the far magnetotail, and a fine resolution of $0.25 R_{E}$ near the magnetopause. 
The simulations analyzed in Chapter 3 employ a higher resolution grid of $0.125 \mathrm{R}_{\mathrm{E}}$ packed in the region $-6<x<10,-10<y<10,-5<z<5 \mathrm{R}_{\mathrm{E}}$ totaling 3,736,800 grid cells; Chapters 4 and 5 employ simulations where the $0.125 \mathrm{R}_{\mathrm{E}}$ resolution is extended to the region $-15<x, y, z<15 \mathrm{R}_{\mathrm{E}}$, totaling $16,286,400$ simulation grid cells. The boundary at $x=-255 \mathrm{R}_{\mathrm{E}}$ is an open boundary where plasma leaves the simulation domain. The other four simulation boundaries at $|y|=|z|=48 \mathrm{R}_{\mathrm{E}}$ are floating boundaries with zero gradients.

Our "base" simulations use constant solar wind values with solar wind temperature $T_{\mathrm{SW}}=$ $232,100 \mathrm{~K}(20 \mathrm{eV})$, IMF strength $20 \mathrm{nT}$, number density $n_{\mathrm{SW}}=20 \mathrm{~cm}^{-3}$, and a solar wind velocity of $\mathbf{v}_{\mathrm{SW}}=-400 \mathrm{~km} / \mathrm{s} \hat{\mathbf{x}}$. We perform distinct simulations with IMF clock angles $\theta_{\mathrm{IMF}}=0^{\circ}$ (parallel), $30^{\circ}, 60^{\circ}, 90^{\circ}, 120^{\circ}, 150^{\circ}, 165^{\circ}$, and $180^{\circ}$ (anti-parallel). The IMF does not have a $B_{x}$ component. Simulations with parameters other than these will be explicitly stated.

The ionospheric electrodynamics (IE) component of the SWMF uses the currents of the MHD simulation at $3.5 \mathrm{R}_{\mathrm{E}}$ to determine the ionospheric currents at a radial distance of 1.017 $\mathrm{R}_{\mathrm{E}}$ using the conservation of electric charge. These ionospheric currents are used to determine the ionospheric electric field $\mathbf{E}$ from the ionospheric conductance tensor $\boldsymbol{\sigma}$, as related in Eq. (1.2). The potential is calculated from these electric fields on a $181 \mathrm{x} 181$ spherical grid where the geomagnetic latitude and longitude are discretized into $1^{\circ}$ increments. Constant Pederson, Hall and field line conductances of 5, 5 and 5000 mhos are used, respectively. We note that the IE component can alternately take the solar radio flux F10.7 index as input and, with an empirical relation, the ionospheric photo-ionization rate is determined which in turn sets the ionospheric conductances, but this feature is not used here. [The F10.7 index is the measured radio emissions at wavelength $10.7 \mathrm{~cm}(2800 \mathrm{MHz})$ that originate from the solar chromosphere. This index correlates well with sunspot number and Ultraviolet irradiance at 


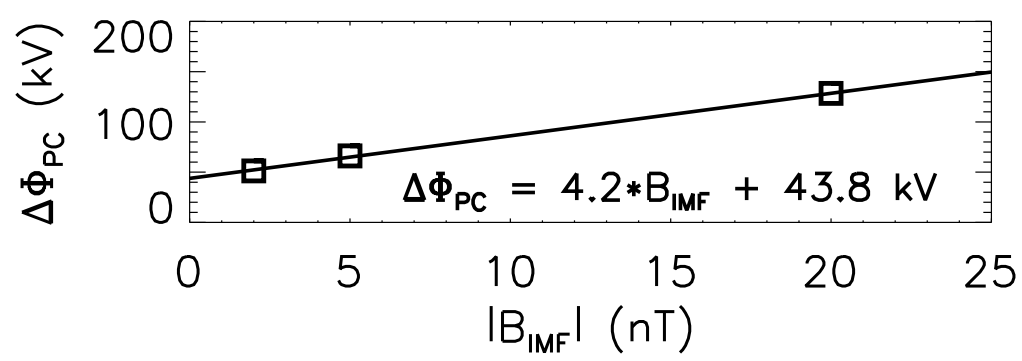

Figure 2.1: Cross polar cap potential difference $\Delta \Phi_{\mathrm{PC}}$ in simulations with $\left|\mathbf{B}_{\mathrm{IMF}}\right|=2,5$ and $20 \mathrm{nT}$ for $\theta_{\mathrm{IMF}}=120^{\circ}$. The potential difference is linear in $\left|\mathbf{B}_{\mathrm{IMF}}\right|$, so the polar cap is not saturated.

Earth; see, e.g., http://www.swpc.noaa.gov/phenomena/f107-cm-radio-emissions).]

\subsubsection{Numerical Test 1: Polar Cap Saturation}

The parameter regime of the solar wind in our base simulations employ atypical IMF strengths and solar wind densities; both quantities are an order of magnitude higher than typically quiet solar wind conditions. This choice is made so the dayside magnetosphere is smaller, and the region of high resolution need not be as large. To ensure that the results are reliable, it is important to check that the polar cap is not saturated, and that reconnection is not saturated for these solar wind parameters (Lopez et al., 2010).

We measure the polar cap potential difference in three distinct simulations with $B_{\mathrm{IMF}}=2$, 5, and $20 \mathrm{nT}$. These simulations have no dipole tilt, the IMF clock angle is fixed at $120^{\circ}$, and all other solar wind parameters are the same as before. Figure 2.1 displays the measured cross polar cap potential difference $\Delta \Phi_{P C}$ as a function of $\left|\mathbf{B}_{\mathrm{IMF}}\right| ; \Delta \Phi_{P C}$ is an output of the IE component. The plot clearly reveals that the potential difference remains linear as a function of IMF strength, implying the polar cap is not saturated for the chosen solar wind parameters. For comparison, Lopez et al. (2010), using LFM simulations, showed that the polar cap potential difference remains linear for $B_{\mathrm{IMF}}<11.25 \mathrm{nT}$ with solar wind density $n_{\mathrm{SW}}=5 \mathrm{~cm}^{-3}$. However, the IMF strength cutoff increases to $B_{\mathrm{IMF}}=15 \mathrm{nT}$ 
when $n_{\mathrm{SW}}=8 \mathrm{~cm}^{-3}$ is used with solar wind speeds of $400 \mathrm{~km} / \mathrm{s}$ and ionospheric Pedersen conductance of 5 mhos. As our simulations employ the same solar wind speed and a much larger solar wind density $n_{\mathrm{SW}}=20 \mathrm{~cm}^{-3}$, it is reasonable to expect that the IMF cutoff will increase as well and we find the polar cap to not be saturated for $B_{\mathrm{IMF}}=20 \mathrm{nT}$. Our measured viscous potential (the $y$-intercept) is $43.8 \mathrm{kV}$, consistent with the trend seen by Lopez et al. (2010), where the measured viscous potentials increase from 33.9 to $41.9 \mathrm{kV}$ when $n_{\mathrm{SW}}=5 \mathrm{~cm}^{-3}$ is increased to $8 \mathrm{~cm}^{-3}$, respectively. We are therefore confident that the polar cap is not saturated for our base simulation parameters.

\subsubsection{Numerical Test 2: Numerical vs. Explicit Dissipation}

Plasmas can experience dissipation physically, such as through resistivity which converts magnetic energy to heat. Further, whenever simulations are performed, there is an effective non-physical dissipation that occurs from the specific algorithm used to evolve plasma variables. It is important to control numerical dissipation in simulation studies of reconnection.

The presence of the resistivity $\eta$ in Faraday's Law, Eq. (2.5), gives rise to dissipation of the magnetic field and allows for a magnetic field line's topology to change. This dissipation is called explicit because it is a term included in the physical equations rather than resulting from the numerics. Many global MHD simulations use numerical dissipation to mediate reconnection instead of explicit dissipation. It has been suggested that explicit dissipation can be detrimental to global magnetospheric simulations (Raeder, 1999). Additionally, the magnetosphere is known to be collisionless. However, explicit dissipation is essential for studies involving magnetic reconnection and magnetic topology. For example, previous global simulations revealed plasma flows through the reconnection X-line (Siscoe et al., 2002; Dorelli et al., 2004); some researchers believed this to be spurious due to high dissipation, but later 

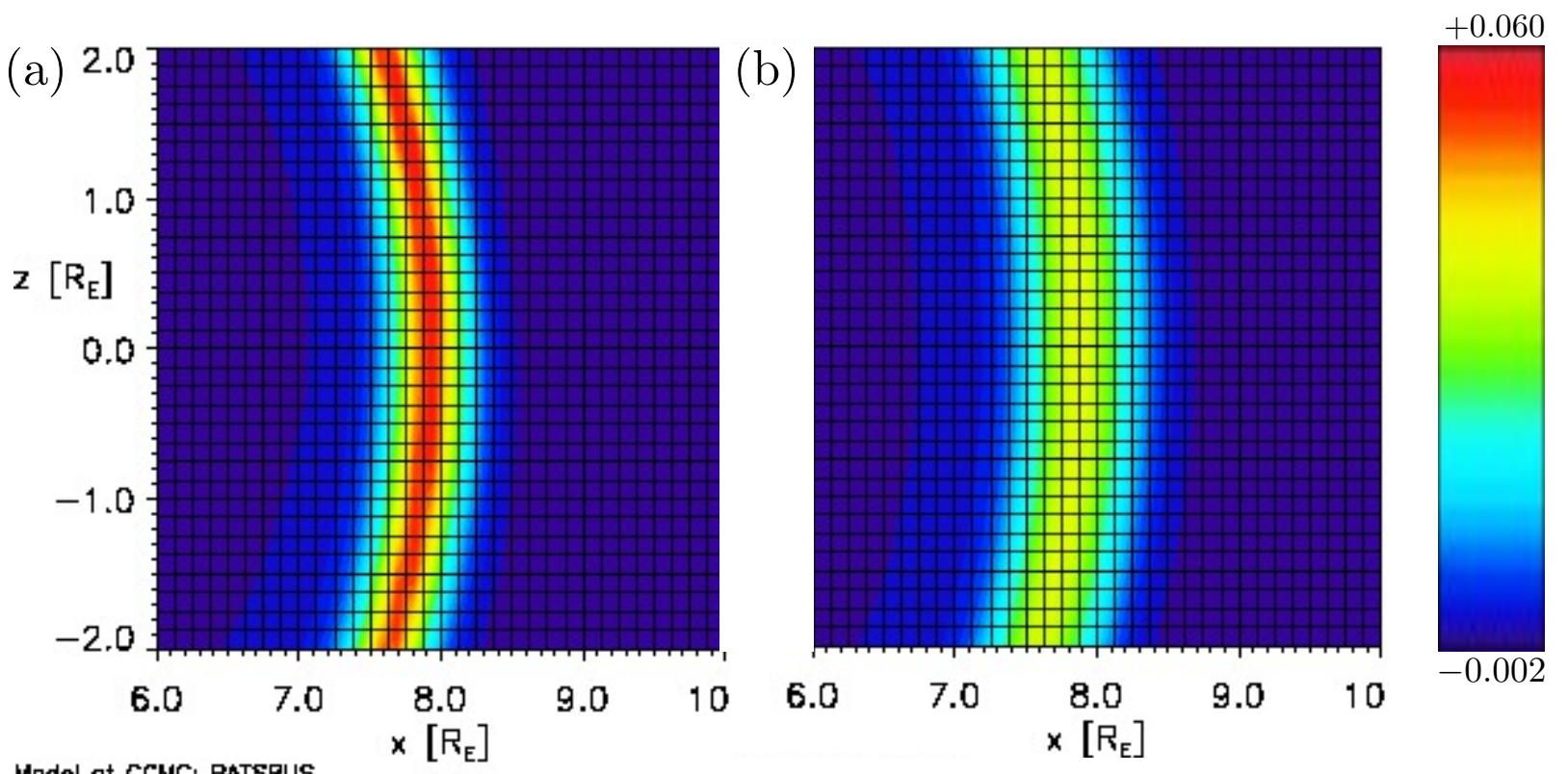

Figure 2.2: Magnetopause current density $J_{y}\left(\mu \mathrm{A} / \mathrm{m}^{2}\right)$ near the subsolar point in high resolution simulations (a) without an explicit resistivity and (b) with an explicit resistivity $\eta / \mu_{0}=6.0 \times$ $10^{10} \mathrm{~m}^{2} / \mathrm{s}$. The current layer broadens in (b), demonstrating the explicit resistivity dominates numerical effects. Solid (black) lines indicate the simulation grid $\left(0.125 \mathrm{R}_{\mathrm{E}}\right)$.

studies showed this flow is a fundamental aspect of asymmetric reconnection (Cassak and Shay, 2007, 2009).

For the present simulations, we employ a uniform explicit resistivity $\eta$. It is known that the magnetosphere is not collisional, but including an explicit resistivity allows for reproducible results that are independent of the numerics. We therefore include an explicit resistivity $\eta / \mu_{0}=6.0 \times 10^{10} \mathrm{~m}^{2} / \mathrm{s}$ in our simulations. We now ensure the explicit resistivity is sufficient to overcome the numerical resistivity. Figure 2.2 displays the current density $J_{y}$ at the subsolar magnetopause in two simulations with $\theta_{\mathrm{IMF}}=180^{\circ}$; one with (a) no explicit resistivity and the other (b) with the specified explicit resistivity. The current layer broadens from six cells to eight cells across, suggesting the explicit resistivity is controlling the dissipation, as desired. This explicit resistivity is similar to the value obtained in a recent study that determined the size of the resistivity necessary for it to control the dissipation 
(G. Toth, private communication).

To facilitate comparisons with previous simulations, we estimate a Lundquist number $S=\mu_{0} c_{A} L / \eta$ for the explicit resistivity simulation with $\theta_{\mathrm{IMF}}=180^{\circ}$; the Lundquist number is the ratio of the convection term to the magnetic diffusion term in Eq. (2.5), where the convection speed is assumed to be the Alfvén speed $c_{A}$. We base the length scale $L$ on the half-length of the reconnecting current sheet in the outflow direction (as opposed to a global length scale), which is $5.35 \mathrm{R}_{\mathrm{E}}$. We substitute the hybrid asymmetric outflow speed $c_{A, a s y m}$, given by Eq. (1.21), for the Alfvén speed $c_{A}$, where the subscripts "1" and "2" indicate quantities measured in the magnetosphere and magnetosheath, respectively. The magnetic fields and densities, measured immediately upstream of the reconnecting current sheet in the Earthward and sunward directions, are $B_{1}=116 \mathrm{nT}, B_{2}=90 \mathrm{nT}, n_{1}=10 \mathrm{~cm}^{-3}$ and $n_{2}=57 \mathrm{~cm}^{-3}$, giving $c_{A, \text { asym }} \simeq 380 \mathrm{~km} / \mathrm{s}$. The resulting Lundquist number based on these quantities and our chosen explicit resistivity is $S \simeq 210$. A benefit of choosing the explicit resistivity this large is that we do not expect FTEs (Russell and Elphic, 1978; Biskamp, 1986) to spontaneously form at the dayside magnetopause in our global magnetospheric simulations. While FTEs do occur at the magnetopause, they would needlessly complicate the present fundamental physics study on field line topology. 


\section{Chapter 3}

\section{Tracing Magnetic Separators}

A number of methods have been developed to locate magnetic separators. The eigenvectors of the $3 \times 3 \nabla \mathbf{B}$ tensor at a magnetic null, i.e., the location where $\mathbf{B}=0$, determine the local magnetic field geometry (Greene, 1988; Lau and Finn, 1990; Parnell et al., 1996). Other methods determine the magnetic separator globally. One method takes an array of IMF lines in the solar wind, and convects them with the solar wind flow; if the field line reconnects, it has crossed the separator, so one can find the separator's location (Dorelli et al., 2007; Ouellette et al., 2010). Simulations without a dipole tilt or no $B_{x}$ component to the IMF have a high degree of symmetry, resulting in a separator that lies on the sun-Earth line. Therefore, the last closed field line along the sun-Earth line has also been used as an approximation of the separator, as this field line closely approaches the magnetic nulls in global magnetospheric simulations [Dorelli et al. (2007) used this method for a northward IMF orientation; Hu et al. (2009) determined the separator for both northward and southward IMF orientations]. The separator has also been located by finding where different magnetic

topologies meet (Laitinen et al., 2006, 2007; Dorelli and Bhattacharjee, 2008, 2009). Haynes and Parnell (2010) developed an iterative technique to map the separator using rings along 
the separatrix eigenvectors of the $\nabla \mathbf{B}$ tensor. Another study (Moore et al., 2008) inferred reconnection geometries from deflections in streamlines at the magnetopause. A few of these studies have investigated separators as a function of IMF clock angle (Laitinen et al., 2007; Hu et al., 2009; Ouellette et al., 2010). However, each of these techniques has its drawbacks.

Here, we present a simple, efficient and accurate algorithm to map magnetic separators in global magnetospheric simulations at the dayside magnetopause for arbitrary IMF clock angle. We verify the technique using exact solutions for an analytic model involving the superposition of uniform and dipolar magnetic fields. Then, we trace separators in global magnetohydrodynamic (MHD) simulations for various IMF clock angles, and show that the last closed field line does not map the separators for southward IMF. We discuss trends in magnetic null locations and magnetic separators, making comparisons to the analytical field model.

The layout of this chapter is as follows. In section 3.1, we present and verify a new method for tracing magnetic separators. The results of finding locations of magnetic nulls and tracing separators for different IMF clock angles are discussed in section 3.2. The results are summarized and potential applications are discussed in Section 3.3.

\subsection{A Technique for Finding Separators}

\subsubsection{Technique Description}

The separator tracing algorithm exploits the fact that magnetic nulls are the endpoints of magnetic separators. A schematic diagram of the tracing process is shown in Fig. 3.1. The two magnetic nulls are found using existing techniques, represented by (red) X's at the endpoints. A hemisphere, represented by a dashed semicircle, is centered around the northern 


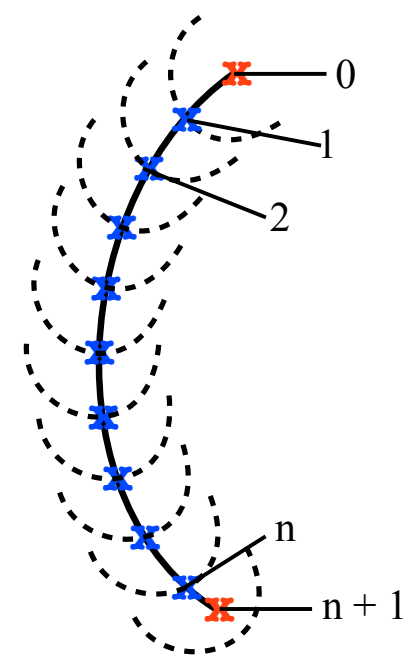

Figure 3.1: Schematic diagram of the separator tracing algorithm.

null, labeled 0 (the choice of starting null is arbitrary). At many points on the hemisphere's surface, the magnetic field is traced in both directions to determine its topology (solar wind, closed, or one of two open topologies). The point at which all topologies meet is where the separator intersects the hemisphere; we mark this intersection with a (blue) $\mathrm{x}$ and label it location 1. A new hemisphere is centered at location 1 and the separator's intersection with this new hemisphere is determined similarly. This new intersection is marked by another (blue) $\mathrm{x}$, labeled as location 2 . This process is repeated until the southern null, labeled $\mathrm{n}+1$, is inside a hemisphere. The separator is mapped by connecting the nulls to the individual separator locations in order $(0$ through $n+1)$, sketched as the solid (black) line. We note an alternate algorithm would be to initially find a single null and perform the above iterative procedure while checking inside each hemisphere for another null at each step, but we do not pursue this further.

We now provide a more detailed description of the steps in this technique. To locate the magnetic nulls, we use the method described by Haynes and Parnell (2007). This method flags those simulation grid cells where all three components of the magnetic field change sign, 
since global minima of the magnetic field magnitude $|\mathbf{B}|$ do not faithfully locate magnetic nulls since nulls may not fall exactly at a grid cell's corner. The field is then linearized within these flagged cells and a Newton-Raphson iteration algorithm is used to locate magnetic nulls at sub-grid resolution.

In most of our global magnetospheric MHD simulations, described in Chapter 2, this method returns a single northern null and southern null. In one, multiple nulls are identified, though the nulls in each hemisphere are within $0.25 \mathrm{R}_{\mathrm{E}}$ of each other. To choose a null, we select the location with the lowest $|\mathbf{B}|$ in one of the hemispheres. The simulations have a high degree of symmetry, so the null in the opposite hemisphere is chosen to be closest to the null's reflection. [That is, for a null located at $(x, y, z)$, the reflected null is near $(x,-y,-z)]$. The null identification is verified by plotting field lines in the vicinity of the chosen nulls; magnetic fields with different topologies converge in these regions, as expected.

Having identified the nulls, we proceed to map the separator. Each hemisphere in the iteration has a fixed radius $R_{\mathrm{HS}}$ (hemispheres are used to automatically prevent retracing in the opposite direction). Points on the hemisphere's surface are mapped by a set of angular coordinates $(\phi, \lambda)$, where $\phi$ is the longitude measured from the $+x$-axis in GSM coordinates and $\lambda$ is the latitude. In spherical coordinates, $\lambda=90^{\circ}-\theta$, where $\theta$ is the polar angle measured from the $+z$-axis in GSM.

We use hemispheres with a radius of $1 R_{E}$, and the surface of each hemisphere is discretized into a $N_{\phi} \times N_{\lambda}$ grid; the topology of the magnetic field is determined at each grid point (we use a $61 \times 61$ grid). To calculate magnetic topology, we use the Kameleon software package developed at NASA's Community Coordinated Modeling Center (CCMC). We perform a bi-directional trace of the magnetic field at each point on the surface to ascertain how the magnetic field line may, or may not, map to Earth and determine its topology. A 
field line is closed if it maps to within $5 \mathrm{R}_{\mathrm{E}}$ of the origin in both directions and is solar wind if both directions do not. Open field lines map to within $5 \mathrm{R}_{\mathrm{E}}$ of the origin in only one direction. The particular open topology, northern or southern, is determined by checking the $z$-coordinate of the side mapping to within $5 \mathrm{R}_{\mathrm{E}}$. For example, if the portion of field line mapping to within $5 \mathrm{R}_{\mathrm{E}}$ has a negative $z$-coordinate, then the field line is a southern-open field line. Conversely, a northern-open field line maps to a positive $z$-coordinate. Each point on the hemisphere is coded by its topology. An example is in Fig. 3.2; closed magnetic fields are colored red, solar wind magnetic fields are orange, southern-open fields are black, and northern-open fields are white.

To identify where the four topologies meet, interpolation is usually necessary. The separator lies between the northern and southern half-closed regions. We start by searching through the topological map for the locations where these regions are closest. In Fig. 3.2, the two closest points are at $\left(0^{\circ},-30^{\circ}\right)$ and $\left(-20^{\circ},-28^{\circ}\right)$. We find the topology of the field line through the midpoint of the line connecting these points. Then, the topology is measured at points along the line perpendicular to this line until a change in topology is measured. The separator location is defined as the average of the two points with differing topologies. Figure 3.2 displays a (black) asterisk at its approximate separator location which reasonably estimates where the four topologies meet.

The separator location is used as the center of the subsequent hemisphere. If the separator intersects the $k$-th hemisphere at longitude and latitude $\left(\phi_{k}, \lambda_{k}\right)$, the coordinates of the next hemisphere's center $\mathbf{r}_{k+1}$ are

$$
\mathbf{r}_{k+1}=\mathbf{r}_{k}+\mathbf{r}\left(R_{\mathrm{HS}}, \phi_{k}, \lambda_{k}\right)
$$




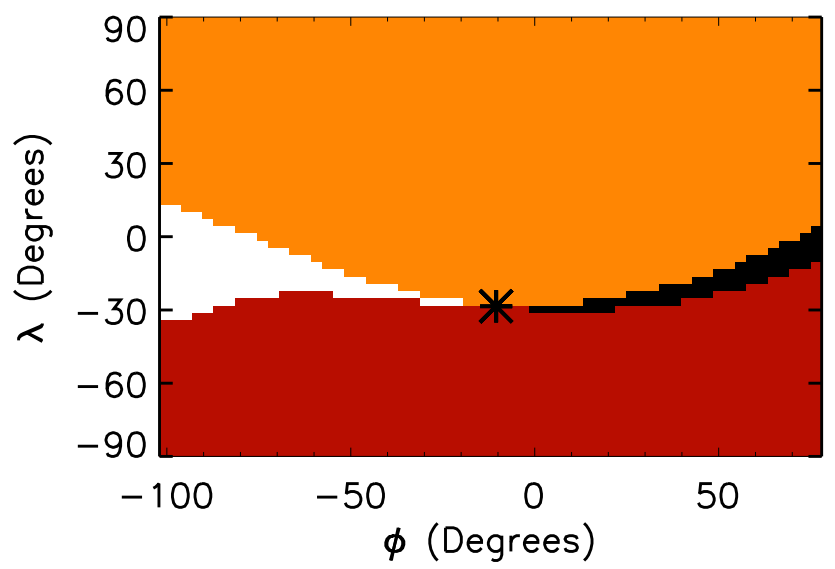

Figure 3.2: Sample topology map for $\theta_{\mathrm{IMF}}=30^{\circ}$ for a hemisphere centered at $\mathbf{r}=(3.16,1.87,8.01) \mathrm{R}_{\mathrm{E}}$, with radius of $1 \mathrm{R}_{\mathrm{E}}$. Colors denote magnetic topology: red are closed terrestrial fields, orange are solar wind fields, black and white are open fields that terminate at the south and north poles of Earth, respectively. The black asterisk marks the approximate separator location.

where $\mathbf{r}_{k}$ is the center of the $k$-th hemisphere, and $\mathbf{r}\left(R_{\mathrm{HS}}, \phi_{k}, \lambda_{k}\right)$ is the separator's location on the $k$-th hemisphere in spherical coordinates relative to $\mathbf{r}_{k}$. A range of $\left[\phi_{k}-90^{\circ}, \phi_{k}+90^{\circ}\right]$ is used as the longitude of $k+1$-st hemisphere; this explains why the horizontal axis in Fig. 3.2 is not centered around $\phi=0^{\circ}$. An arbitrary degree of accuracy can be obtained by decreasing the radius $R_{\mathrm{HS}}$ and/or increasing the number of grid points $N_{\phi}, N_{\lambda}$ on the hemispheres.

The method described here has some similarities to the four field junction method by Laitinen et al. (2006). This method calculates the magnetic topology at every point on a Cartesian grid near regions where the separator is thought to exist. The separator is approximated by locations where all four topologies are within 3 grid cells of each other, resulting in a ribbon-like structure at the dayside magnetopause. Our method does not require a priori knowledge of the separator's location, as it starts from the nulls and traces the complete dayside portion of the separator. It is also computationally inexpensive, since the magnetic topology is calculated on a number of surfaces rather than a volume at the dayside magnetopause. 


\subsubsection{Verification with Vacuum Superposition}

To test the technique in Section 3.1.1, we use a simple magnetic field model with analytic solutions for the nulls and separators. Consider a dipolar magnetic field $\mathbf{B}_{\mathrm{D}}$ superposed with a uniform background magnetic field $\mathbf{B}_{\mathrm{IMF}}$. The vacuum superposition magnetic field $\mathbf{B}_{\mathrm{VS}}$ is given by

$$
\mathbf{B}_{\mathrm{VS}}(\mathbf{r})=\mathbf{B}_{\mathrm{D}}(\mathbf{r})+\mathbf{B}_{\mathrm{IMF}}
$$

where

$$
\mathbf{B}_{\mathrm{D}}(\mathbf{r})=\frac{3(\mathbf{M} \cdot \hat{\mathbf{r}}) \hat{\mathbf{r}}-\mathbf{M}}{r^{3}}
$$

$\mathbf{M}$ is Earth's magnetic dipole moment, and $\mathbf{r}$ is the position vector. The IMF in GSM coordinates is

$$
\mathbf{B}_{\mathrm{IMF}}=B_{\mathrm{IMF}}\left(\sin \theta_{\mathrm{IMF}} \hat{\mathbf{y}}+\cos \theta_{\mathrm{IMF}} \hat{\mathbf{z}}\right)
$$

which assumes no $x$ component for simplicity. The positions $\mathbf{r}_{\text {Null }}$ of the magnetic nulls satisfy

$$
\mathbf{B}_{\mathrm{D}}\left(\mathbf{r}_{\mathrm{Null}}\right)+\mathbf{B}_{\mathrm{IMF}}=0
$$

For the chosen form of $\mathbf{B}_{\mathrm{IMF}}$ and using no dipole tilt, the nulls in spherical coordinates are at $\mathbf{r}_{\mathrm{Null}}=\left(r_{\mathrm{Null}}, \phi_{\mathrm{Null}}= \pm 90^{\circ}, \pm \lambda_{\mathrm{Null}}\right)($ Yeh, 1976; Hu et al., 2009), where

$$
r_{\mathrm{Null}}=\left(\frac{M}{2 B_{\mathrm{IMF}}}\right)^{1 / 3}\left[\cos \theta_{\mathrm{IMF}}+\sqrt{8+\cos ^{2} \theta_{\mathrm{IMF}}}\right]^{1 / 3}
$$

and

$$
\lambda_{\mathrm{Null}}=\tan ^{-1}\left(\frac{3 \cos \theta_{\mathrm{IMF}}+\sqrt{8+\cos ^{2} \theta_{\mathrm{IMF}}}}{4 \sin \theta_{\mathrm{IMF}}}\right) .
$$




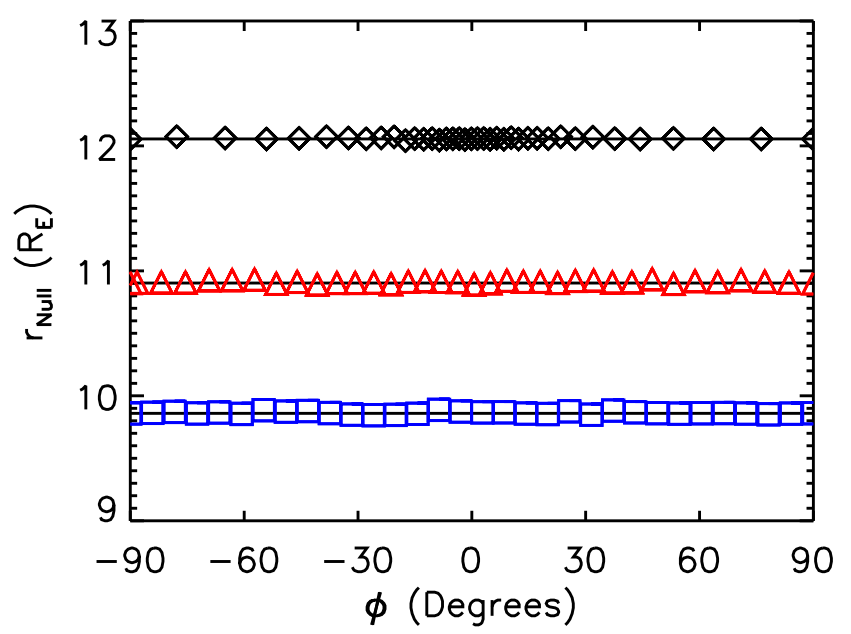

Figure 3.3: Verification of the separator tracing algorithm for $\theta_{\mathrm{IMF}}=30^{\circ}$ (black diamonds), $90^{\circ}$ (red triangles), and $150^{\circ}$ (blue squares) using vacuum superposition. The exact solutions for the separator are shown as solid (black) lines.

In GSM coordinates, $\mathbf{r}_{\mathrm{Null}}=\left(x_{\mathrm{Null}}, y_{\mathrm{Null}}, z_{\mathrm{Null}}\right)$ with

$$
\begin{gathered}
x_{\mathrm{Null}}=0 \\
y_{\mathrm{Null}}=r_{\mathrm{Null}}\left(\frac{3+\sin ^{2} \theta_{\mathrm{IMF}}-\cos \theta_{\mathrm{IMF}} \sqrt{\cos ^{2} \theta_{\mathrm{IMF}}+8}}{6}\right)^{1 / 2},
\end{gathered}
$$

and

$$
z_{\mathrm{Null}}=r_{\mathrm{Null}}\left(\frac{2+\cos ^{2} \theta_{\mathrm{IMF}}+\cos \theta_{\mathrm{IMF}} \sqrt{\cos ^{2} \theta_{\mathrm{IMF}}+8}}{6}\right)^{1 / 2}
$$

The nulls lie in the dawn-dusk plane $[x=0]$, as there is no $B_{x}$ component to the IMF field. The separator is a semicircular arc of radius $r_{\text {Null }}$ connecting the two nulls (Cowley, 1973; Yeh, 1976; Hu et al., 2009). To motivate that this is the case, note that for pure southward IMF, the separator is a circle in the ecliptic plane. For other clock angles, the separator rotates out of the ecliptic plane by $\lambda_{\text {Null }}$ without changing its shape.

We use the technique from Section 3.1.1 to trace separators in vacuum superposition with $\theta_{\mathrm{IMF}}=30^{\circ}, 90^{\circ}$, and $150^{\circ}$ for a system with $\mathbf{M}=-5.13 \times 10^{4} \mathrm{nT} \mathrm{R}_{\mathrm{E}}^{3} \hat{\mathbf{z}}$ and $B_{\mathrm{IMF}}=56 \mathrm{nT}$. 
The tracing algorithm uses hemispheres with radii $R_{\mathrm{HS}}=1 \mathrm{R}_{\mathrm{E}}$. The separator locations are plotted in Fig. 3.3 as (black) diamonds for $\theta_{\mathrm{IMF}}=30^{\circ}$, (red) triangles for $90^{\circ}$, and (blue) squares for $150^{\circ}$. The exact solutions for the separator from Eq. (3.6) are plotted as solid (black) lines. The measured separator locations agree exceedingly well with the exact solutions.

To test the accuracy of the algorithm, we repeat the tracing using hemispheres with radii $R_{\mathrm{HS}}=5 \mathrm{R}_{\mathrm{E}}$ (not shown). As expected, the agreement is better with hemispheres of smaller radii. The scatter of the separator locations from the exact solution in Eq. (3.6), measured as the average absolute difference between the measured separator radius and $r_{\text {Null }}$, is $\sim 75 \%$ lower when smaller hemispheres are used.

\subsection{Results}

Here, we describe the results of finding nulls and tracing separators in the global, resistive MHD simulations described in Chapter 2. To develop perspective on the results, we compare the results to nulls and separators in vacuum superposition given by Eqs. (3.6) and (3.7). To make a careful comparison, we do not use the simulation values of $\mathbf{M}=-3.11 \times 10^{4} \mathrm{nT} \mathrm{R} \mathrm{R}_{\mathrm{E}}^{3} \hat{\mathbf{z}}$ for the magnetic moment of Earth and $B_{\mathrm{IMF}}=20 \mathrm{nT}$ because the terrestrial magnetic field is enhanced due to compression by the solar wind and the IMF increases after crossing the bow shock. We find more appropriate values from the MHD simulations. The magnetic field strengths are measured upstream of the current sheet at the subsolar point. To do so, the locations where the magnetopause current drops to $1 / e$ of its maximum on the sun-Earth line is found for both sides of the sheet. On the magnetospheric side, the magnetic field averages a $65 \%$ increase over Earth's nominal dipole field in our simulations for all clock 
Table 3.1: The $(x, y, z)$ coordinates (in GSM) of determined magnetic nulls in global magnetosphere simulations and in vacuum superposition with $\mathbf{M}=-5.13 \times 10^{4} \mathrm{nT} \mathrm{R}_{\mathrm{E}}^{3} \hat{\mathbf{z}}$ and $B_{\mathrm{IMF}}=56 \mathrm{nT}$.

\begin{tabular}{ccc}
\hline Clock Angle & MHD Nulls $\left(\mathrm{R}_{\mathrm{E}}\right)$ & Vacuum Nulls $\left(\mathrm{R}_{\mathrm{E}}\right)$ \\
\hline $0^{\circ}$ & $(0.08,0.00, \pm 10.28)$ & $(0.00,0.00, \pm 12.24)$ \\
\hline $30^{\circ}$ & $(-0.10, \pm 2.99, \pm 9.99)$ & $(0.00, \pm 4.08, \pm 11.34)$ \\
\hline $60^{\circ}$ & $(-0.44, \pm 5.19, \pm 9.35)$ & $(0.00, \pm 7.18, \pm 9.06)$ \\
\hline $90^{\circ}$ & $(-0.41, \pm 7.70, \pm 7.96)$ & $(0.00, \pm 8.91, \pm 6.29)$ \\
\hline $120^{\circ}$ & $(-0.92, \pm 9.55, \pm 5.83)$ & $(0.00, \pm 9.56, \pm 3.78)$ \\
\hline $150^{\circ}$ & $(-2.16, \pm 11.03, \pm 3.46)$ & $(0.00, \pm 9.70, \pm 1.75)$ \\
\hline $165^{\circ}$ & $(0.93, \pm 10.11, \pm 1.89)$ & $(0.00, \pm 9.71, \pm 0.85)$ \\
\hline
\end{tabular}

angles, so we employ $\mathbf{M}=-5.13 \times 10^{4} \mathrm{nT} \mathrm{R}_{\mathrm{E}}^{3} \hat{\mathbf{z}}$. On the magnetosheath side, $B_{\mathrm{IMF}} \simeq 56 \mathrm{nT}$ for all clock angles. These are the values we employ for the vacuum superposition fields.

\subsubsection{The Magnetic Nulls}

We find the magnetic nulls by employing an established method (Haynes et al., 2007) and their GSM locations are listed in Table 3.1 for the MHD simulations and for the vacuum superposition fields. The latter are in close agreement with Eqs. (3.8)-(3.10). The measured magnetic field strength at each of the locations identified as nulls is $0.1 \mathrm{nT}$ or lower. Nulls for $\theta_{\mathrm{IMF}}=180^{\circ}$ are not reported as there are an infinite number of them in the ecliptic plane. As an example, the magnetic nulls for the $\theta_{\mathrm{IMF}}=90^{\circ} \mathrm{MHD}$ simulation are plotted as purple spheres in Fig. 3.4, showing an (a) Earthward and (b) oblique view for perspective. The Earth is depicted as the green sphere (to scale).

The location of the magnetic nulls exhibit a few interesting trends as a function of IMF clock angle. The nulls in vacuum superposition have a range of $12.3 \geq r_{\text {Null }} \geq 9.7 \mathrm{R}_{\mathrm{E}}$ for $0^{\circ} \leq \theta_{\mathrm{IMF}} \leq 180^{\circ}$, whereas the nulls found in MHD have a nearly constant $r_{\text {Null }} \simeq 10.5 \mathrm{R}_{\mathrm{E}}$. The trend in $r_{\text {Null }}$ differs because the magnetopause is located where the magnetospheric magnetic pressure balances the solar wind ram pressure in MHD. Our MHD simulations all 
(a)

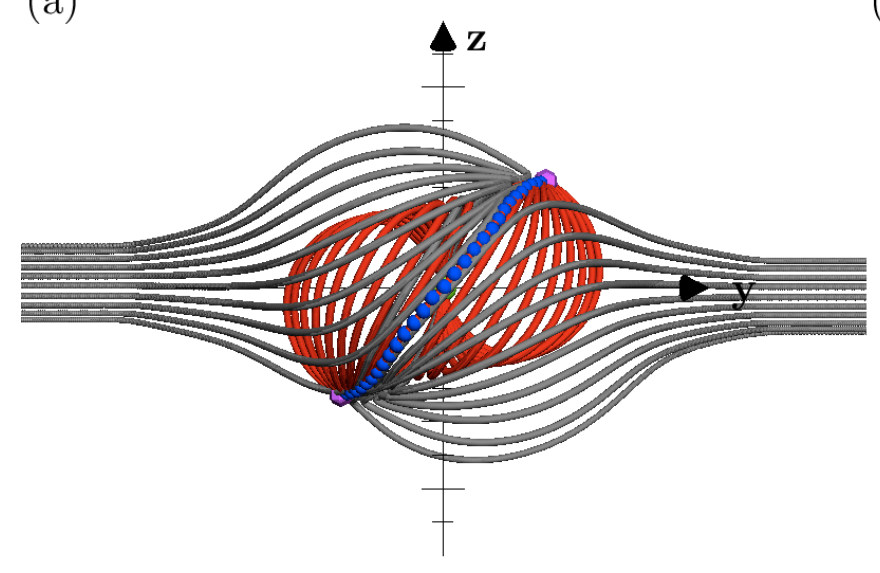

(b)

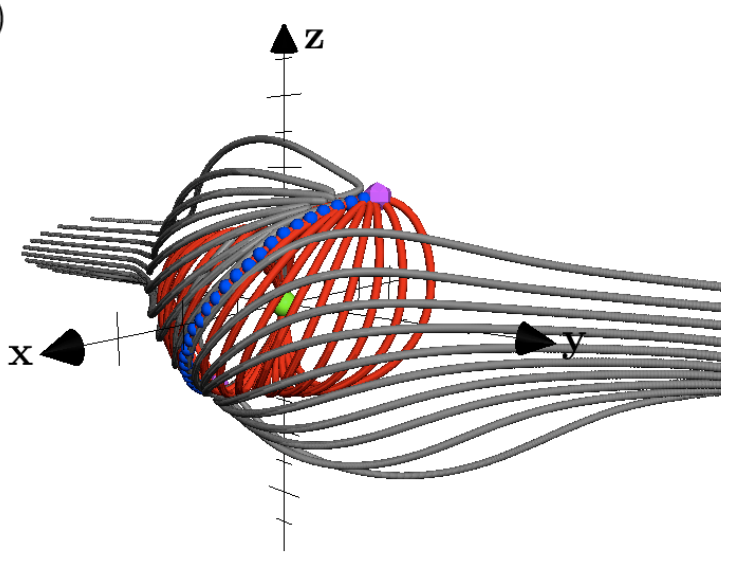

Figure 3.4: Results of the present algorithm in the MHD simulation with $\theta_{\mathrm{IMF}}=90^{\circ}$ looking (a) Earthward and (b) at an oblique angle for perspective. Magnetic nulls are enclosed by (purple) spheres and Earth is the (green) sphere at the origin (to scale). The last closed magnetic field lines in the ecliptic plane are displayed in red and adjacent half-closed topologies are displayed in gray.

have the same solar wind conditions, which explains why $r_{\text {Null }}$ remains constant in MHD. Vacuum superposition is only a magnetic field model and does not capture this solar wind physics. The magnetopause shrinks as $\theta_{\mathrm{IMF}}$ increases because the IMF $B_{z}$ component becomes increasingly negative, enabling the IMF to penetrate further into the model magnetosphere.

Figure 3.5 displays the measured MHD coordinates of the nulls as asterisks and the solid lines as the predicted values for vacuum superposition from Eqs. (3.8)-(3.10) as a function of $\theta_{\text {IMF. }}$. The $y$ and $z$ coordinates of the nulls follow qualitatively similar trends for both vacuum superposition and MHD and are within $2 \mathrm{R}_{\mathrm{E}}$ of each other for all clock angles. In Fig. 3.5(b), the $y$ coordinate increases from zero as $\theta_{\mathrm{IMF}}$ increases, and the nulls move out of the noon-midnight plane. The $z$ coordinate decreases to zero for increasing clock angle, as seen in Fig. 3.5(c). This is because the nulls are located at the magnetic cusps for northward IMF and are in the ecliptic plane for southward IMF.

The $x$-coordinates of the nulls displayed in Fig. 3.5(a) do not follow the same trend as in 


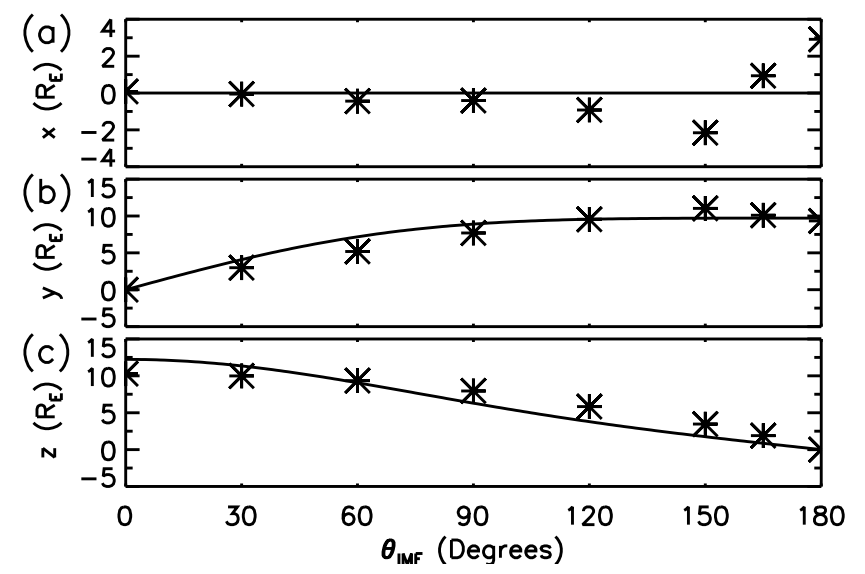

Figure 3.5: Plots of magnetic null (a) $x$-coordinate, (b) $y$-coordinate, and (c) $z$-coordinate as a function of IMF clock angle $\theta_{\text {IMF }}$. Solid lines display vacuum superposition prediction from Eqs. (3.8)(3.10) and asterisks are the coordinates of nulls in the MHD simulations.

vacuum superposition. The nulls in vacuum superposition are in the dawn-dusk plane $[x=0]$ for all $\theta_{\mathrm{IMF}}$ as there is no $B_{x}$ component of the IMF [see Eq. (3.8)]. In the MHD simulations, the nulls are near $x=0$ for small clock angles, but migrate towards the nightside as $\theta_{\mathrm{IMF}}$ increases towards $150^{\circ}$. Interestingly, this trend is broken for $\theta_{\mathrm{IMF}}=165^{\circ}$ which has a null with a $+x$-coordinate.

One might suggest the migration of the nulls to the nightside results from the draping of the IMF over the magnetosphere. Draping causes the IMF to be oriented sunward in the southern hemisphere and tailward in the northern hemisphere for northward IMF, with the opposite being true for southward IMF. However, this effect would make the nulls migrate opposite to the observed direction, so draping cannot explain the migration of the nulls' $x$-coordinate. We conclude that there is no simple explanation of the trend in the $x$-coordinate of the nulls, but this is not surprising since null locations are dependent on the shape of the magnetopause, which has a multi-parameter dependence on upstream solar wind conditions (Lu et al., 2011; Liu et al., 2012). 


\subsubsection{The Magnetic Separators}

The separator tracing method described in Section 3.1 is used to trace the dayside separators for the MHD simulations. We start from the magnetic nulls described in the previous section and use hemispheres with radii of $R_{\mathrm{HS}}=1 \mathrm{R}_{\mathrm{E}}$ to trace the separators.

Care must be taken in tracing the separator for $\theta_{\mathrm{IMF}}=180^{\circ}$ due to the infinite number of nulls in the ecliptic plane. We start by centering a sphere at the subsolar point $\mathbf{r}_{\mathrm{Null}}=$ (7.87, 0.00, 0.00) $\mathrm{R}_{\mathrm{E}}$. We center the hemisphere at the subsolar point, and the hemisphere is discretized into the same $N_{\phi} \times N_{\lambda}$ grid as described in Section 3.1.1. The hemisphere's coordinates span longitude $0^{\circ} \leq \phi \leq 180^{\circ}$ and latitude $-90^{\circ} \leq \lambda \leq 90^{\circ}$. The chosen longitude range only traces the portion of the separator duskward of the subsolar point. The algorithm iteratively marches in the ecliptic plane until it no longer detects a merging location, ending at $\mathbf{r}=(2.93,9.33,0.00) \mathrm{R}_{\mathrm{E}}$. The dawnward portion of the separator is traced likewise by forcing the hemisphere to have a longitude range of $-180^{\circ} \leq \phi \leq 0^{\circ}$, ending at $\mathbf{r}=(2.93,-9.33,0.00) \mathrm{R}_{\mathrm{E}}$. The resulting separator is stitched together with the subsolar point as the center of each portion.

An example of a traced separator for $\theta_{\mathrm{IMF}}=90^{\circ}$ is shown in Fig. 3.4, with the blue spheres denoting the intersection of the separator with the hemispheres from the iterative technique described in Section 3.1.1. For perspective, the last closed field lines in the ecliptic plane are shown in red and the adjacent half-closed field lines are shown in gray.

\subsubsection{Comparison with the Last Closed Field Line}

The last closed field line on the sun-Earth line has been used to approximate the magnetic separator since it closely approaches both magnetic nulls [northward IMF orientation: (Dorelli et al., 2007); southward and northward IMF orientations: Hu et al. (2009)]. 
(a)

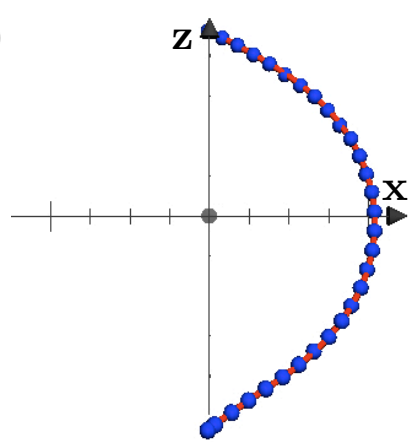

(c)

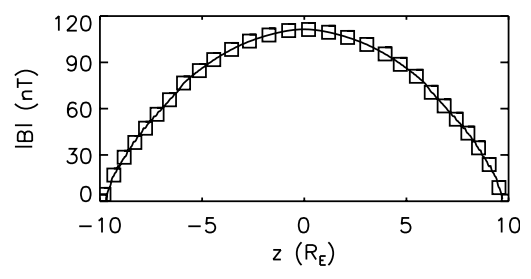

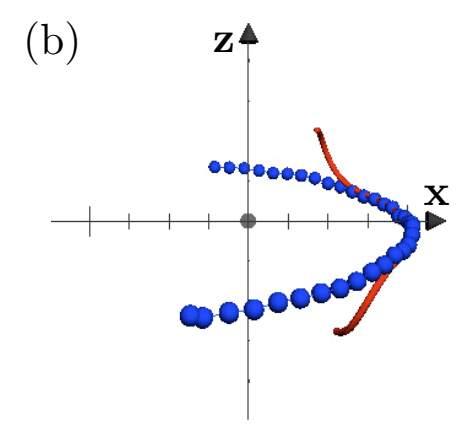

(d)

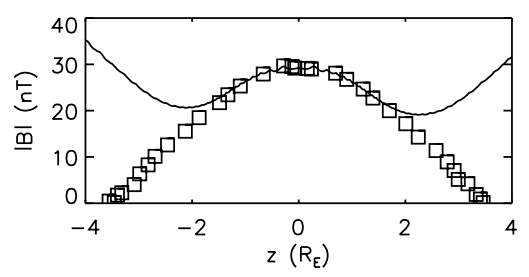

Figure 3.6: Comparison between traced separators and the last closed field line in global MHD simulations. The last closed field line is a solid (red) line and the determined separator locations are (blue) spheres for $\theta_{\mathrm{IMF}}$ of (a) $30^{\circ}$ and (b) $150^{\circ}$. The magnetic field strength $|\mathbf{B}|$ as a function of $z$-coordinate along the separator is a solid line for the last closed field line and as squares for the traced separator for $\theta_{\mathrm{IMF}}$ (c) $30^{\circ}$ and (d) $150^{\circ}$.

We compare the traced separators with the last closed field lines on the $x$-axis for two different clock angles. Figures 3.6(a) and (b) show the last closed field line as a solid (red) line and the individual locations determined by the method described in Section 3.1 as (blue) spheres for $\theta_{\mathrm{IMF}}=30^{\circ}$ and $150^{\circ}$, respectively. The traced separator and last closed field line are nearly identical in (a), where the IMF has a northward $B_{z}$ component. In contrast, the two have a large deviation in (b), where the IMF has a southward $B_{z}$ component. Panels (c) and (d) display $|\mathbf{B}|$ as a function of separator $z$-coordinate along the separator, with the last closed field line shown as a solid (black) line and the locations of the traced separator plotted as squares for the same two cases. The last closed field line and the traced separator are coincident in (c) and, importantly, both connect with the magnetic nulls. In (d), the traced separator closely agrees with the last closed field line near the subsolar point, but only the traced separator connects with the magnetic nulls, while the last closed field line diverges 
(a)

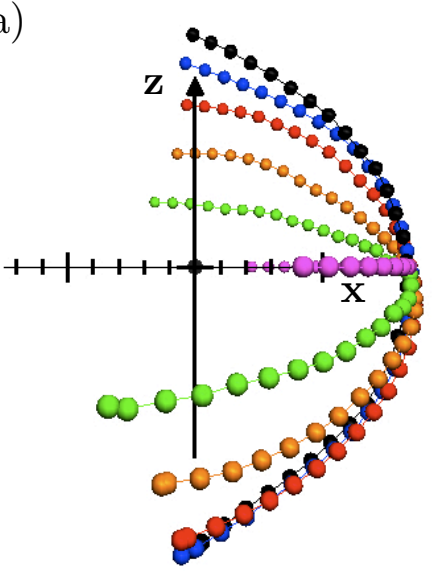

(b)

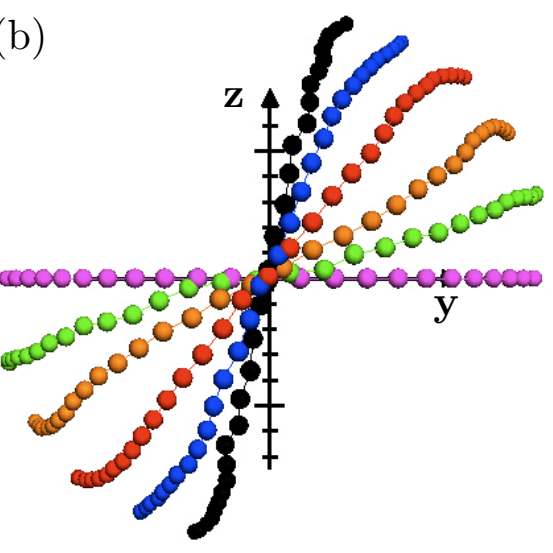

Figure 3.7: Plot of separators in global MHD simulations for $\theta_{\mathrm{IMF}}=30^{\circ}$ (black), $60^{\circ}$ (blue), $90^{\circ}$ (red), $120^{\circ}$ (orange), $150^{\circ}$ (green), $180^{\circ}$ (purple) looking (a) duskward and (b) earthward.

strongly. More generally, we find that both methods agree near the subsolar point for all $\theta_{\mathrm{IMF}}$, but as $\theta_{\mathrm{IMF}}$ increases from $90^{\circ}$, the last closed field line increasingly deviates from the traced separators. Therefore, the last closed field line does not accurately map the entire separator for southward $B_{z}$ in our simulations. While the last closed field line is accurate for northward $B_{z}$, the method of Section 3.1.1 works for any clock angle.

\subsubsection{Clock angle dependence of MHD Separators}

We now turn to comparing separators for different clock angles. The separators traced for clock angles $30^{\circ}$ through $180^{\circ}$ are displayed in Fig. 3.7. Panel (a) displays the separators looking duskward along the $y$-axis and panel (b) displays the separators looking Earthward along the $x$-axis. Each separator is roughly coplanar, and is tilted around the $x$-axis by an amount dependent on the clock angle.

To quantify the structural properties of the separator, we define the separator tilt angle $\kappa$ at the subsolar point measured with respect to the $z$-axis. The tilt angles of the separators are measured using the $B_{y}$ and $B_{z}$ components of the last closed field line at the subsolar 
point:

$$
\kappa_{\mathrm{MHD}}=\tan ^{-1}\left(\frac{B_{y}}{B_{z}}\right) .
$$

To investigate the separator shape as a function of clock angle, we rotate clockwise around the $+x$-axis by $\kappa_{\mathrm{MHD}}$ and display the separator's projection in this rotated plane. The separator's projected coordinates in this plane are given by

$$
\left(\begin{array}{l}
x^{\prime} \\
y^{\prime} \\
z^{\prime}
\end{array}\right)=\left(\begin{array}{ccc}
1 & 0 & 0 \\
0 & \cos \kappa_{\mathrm{MHD}} & -\sin \kappa_{\mathrm{MHD}} \\
0 & \sin \kappa_{\mathrm{MHD}} & \cos \kappa_{\mathrm{MHD}}
\end{array}\right)\left(\begin{array}{l}
x \\
y \\
z
\end{array}\right)
$$

where $x^{\prime}$ points sunward, $y^{\prime}$ is the out-of-plane direction, $z^{\prime}$ is the plane of the separator, and $(x, y, z)$ is the vector for a given location on the separator in GSM coordinates.

Figure 3.8(a) shows the separator's projection in the rotated plane for different IMF clock angles: $\theta_{\mathrm{IMF}}=30^{\circ}$ as $($ black $)$ pluses $\left(\kappa_{\mathrm{MHD}} \simeq 12.6^{\circ}\right), 60^{\circ}$ as $($ red $)$ asterisks $\left(\kappa_{\mathrm{MHD}} \simeq 21.4^{\circ}\right)$, $90^{\circ}$ as $($ blue $)$ diamonds $\left(\kappa_{\mathrm{MHD}} \simeq 40.2^{\circ}\right), 120^{\circ}$ as $($ green $)$ triangles $\left(\kappa_{\mathrm{MHD}} \simeq 62.8^{\circ}\right), 150^{\circ}$ as (purple) squares $\left(\kappa_{\mathrm{MHD}} \simeq 79.4^{\circ}\right), 165^{\circ}$ as (gray) $\mathrm{X}$ 's $\left(\kappa_{\mathrm{MHD}} \simeq 79.0^{\circ}\right)$, and $180^{\circ}$ as $($ green $)$ pluses $\left(\kappa_{\mathrm{MHD}}=90^{\circ}\right)$. The symbol size denotes the location's deviation from the plane, with smaller symbols indicating a larger deviation from the plane.

Figure 3.8(a) simultaneously quantifies three structural features of the magnetic separators. It is clearly seen that the separators maintain a similar shape regardless of $\theta_{\mathrm{IMF}}$. Also, the separators rotate around the magnetopause for increasing $\theta_{\mathrm{IMF}}$, turning clockwise around the $x$-axis. Finally, the symbols indicate that a large portion of the separator is approximately coplanar in the plane defined by $\kappa_{\mathrm{MHD}}$, particularly at the nose of the magnetosphere where the deviation from the plane is $\leq 0.2 \mathrm{R}_{\mathrm{E}}$. The deviation is larger near the 


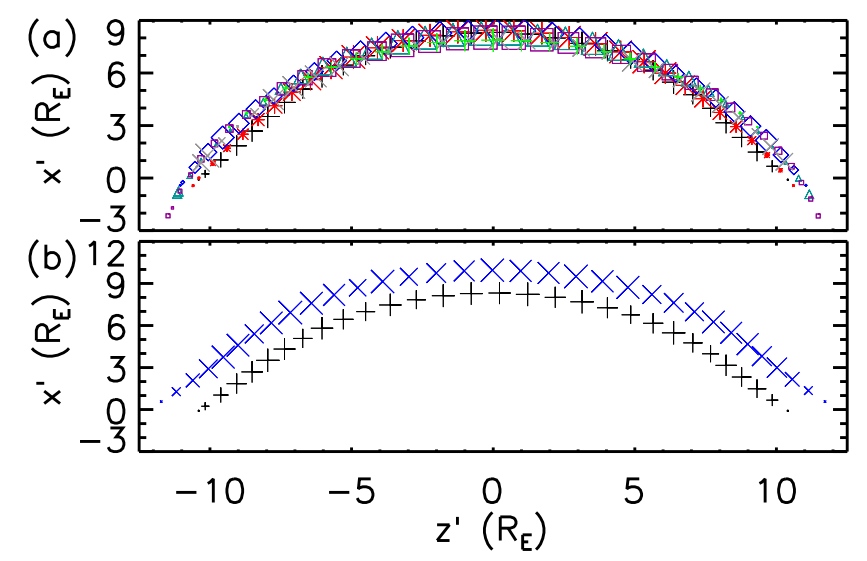

Figure 3.8: MHD separators rotated around $\hat{\mathbf{x}}$ into a common plane. (a) Separators for the different clock angles are: $30^{\circ}$ (black plus), $60^{\circ}$ (red asterisk), $90^{\circ}$ (blue diamond), $120^{\circ}$ (green triangle), $150^{\circ}$ (purple squares), $165^{\circ}$ (grey X), and $180^{\circ}$ (green plus). (b) Plot of MHD separators with $\theta_{\mathrm{IMF}}=30^{\circ}$ with solar wind number density $n=20 \mathrm{~cm}^{-3}$ (black plus) and $n=5 \mathrm{~cm}^{-3}$ (blue $\mathrm{X})$. Symbol size is inversely related to the deviation from the plane.

nulls $\left[\simeq 1.5 R_{E}\right]$, which can clearly be seen in Fig. 3.7(b), where the ends of the separators flare towards the dawn and dusk flanks. This implies that it is not accurate to model separators as lying in the plane of the nulls.

\subsubsection{Comparison with Vacuum Superposition}

To gain perspective on the observed trends in separators in MHD simulations with varying IMF clock angle, we compare them to vacuum superposition separators, although a perfect correlation is not expected. As discussed earlier, MHD separators are mostly coplanar; the vacuum superposition separators are exactly coplanar. Also, the shape of the separator is different between the models. In vacuum superposition, the separator is a circular arc with radius $r_{\text {Null }}$ given by Eq. (3.6). The MHD separators exhibit the well known bullet shape of the magnetopause, as seen in Fig. 3.8.

The separator tilt angle dependence on IMF clock angle is displayed in Fig. 3.9. The black pluses display $\kappa_{\mathrm{MHD}}$ as calculated by Eq. (3.11). For the vacuum superposition separators, Yeh (1976) and $H u$ et al. (2009) showed the separator tilt angle satisfies $\kappa_{\mathrm{VS}}=$ 
$90^{\circ}-\lambda_{\text {Null }}$, with $\lambda_{\text {Null }}$ given by Eq. (3.7), displayed as the dashed (blue) line. Lastly, the solid (red) line shows $\kappa=\theta_{\mathrm{IMF}} / 2$, the angle bisecting the IMF and terrestrial magnetic field, a commonly used estimate, for reference.

The separator tilt angle $\kappa$ increases from $0^{\circ}$ to $90^{\circ}$ for vacuum superposition, MHD, and angle of bisection. The tilt angles for the three $\kappa$ values are relatively close to each other, within about $20^{\circ}$. However, quantitative predictions and trends with clock angle reveal important differences between the models. In vacuum superposition, the tilt angle is consistently larger than the angle of bisection, implying separators that are tilted towards the ecliptic plane. MHD separators are tilted towards the noon-midnight meridional plane for $\theta_{\mathrm{IMF}} \leq 90^{\circ}$, but are tilted towards the ecliptic for $\theta_{\mathrm{IMF}}=120^{\circ}$ and $150^{\circ}$. Therefore, the three models follow similar trends at small and large IMF clock angles, but the MHD separator tilt angle displays significant differences from the models for intermediate clock angles.

\subsubsection{Density Dependence of Separators}

As a preliminary test of the parametric dependence of MHD separator characteristics, we perform a simulation similar to our $\theta_{\mathrm{IMF}}=30^{\circ}$ simulation, only changing solar wind number density to $n=5 \mathrm{~cm}^{-3}$ (from $n=20 \mathrm{~cm}^{-3}$ ). We expect the magnetosphere to expand with this decrease in number density. The location of the magnetopause $R_{\mathrm{MP}}$ on the $x$-axis occurs approximately where the solar wind dynamic pressure balances the magnetosphere's magnetic pressure. As the magnetospheric magnetic field is dipolar with $B_{\mathrm{D}} \propto 1 / r^{3}$, the magnetopause location $R_{\mathrm{MP}}$ depends on density as $R_{\mathrm{MP}} \propto n^{-1 / 6}$. For these simulations, this implies the magnetopause should approximately expand by a factor of $(5 / 20)^{-1 / 6} \simeq 1.26$. The measured values of $R_{\mathrm{MP}}$ from the simulations are $9.94 \mathrm{R}_{\mathrm{E}}$ for $n=5 \mathrm{~cm}^{-3}$ and $8.32 \mathrm{R}_{\mathrm{E}}$ 


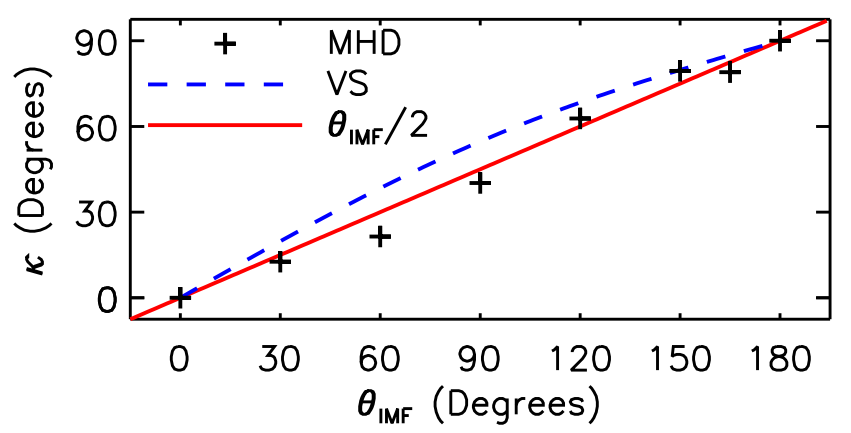

Figure 3.9: Separator tilt angle $\kappa$ at the subsolar point as a function of IMF clock angle $\theta_{\text {IMF. }}$. The solid (red) line is the bisection angle $\theta_{\mathrm{IMF}} / 2$, the dashed (blue) line is for vacuum superposition separators and the pluses are for MHD separators.

for $n=20 \mathrm{~cm}^{-3}$, giving a ratio of $\simeq 1.20$, in good agreement with expectations.

The magnetic nulls of the $n=5 \mathrm{~cm}^{-3}$ simulation are located at $\mathbf{r}_{\mathrm{Null}}=(0.56, \pm 3.16$, $\pm 11.30) \mathrm{R}_{\mathrm{E}}$. The magnetic nulls of the lower density run are sunward and radially outward from their high density counterparts. This behavior is due to the expansion of the magnetosphere with a lower solar wind density. Thus, the magnetic null locations are sensitive to the solar wind density.

The expansion of the magnetosphere also affects separator location. Figure 3.8(b) displays the separators for the two simulations rotated into the principle plane of the separator. The original high density run is displayed as (black) pluses and the low density run as (blue) X's. As expected, the $n=5 \mathrm{~cm}^{-3}$ separator expands outward in the $x^{\prime}-z^{\prime}$ plane. We measure the separator tilt angle using Eq. (3.11); the $n=5 \mathrm{~cm}^{-3}$ simulation has $\kappa_{\mathrm{MHD}} \simeq 12.9^{\circ}$ and $n=20 \mathrm{~cm}^{-3}$ simulation has $\kappa_{\mathrm{MHD}} \simeq 12.6^{\circ}$. Interestingly, despite the expansion of the magnetosphere changing the separator's location, the separator's tilt angle is not strongly dependent on solar wind number density (for the chosen set of simulation parameters). A more complete parametric study to obtain trends in separator morphology is necessary. 


\subsubsection{Dependence on Grid Resolution}

The null locations given in Table 3.1 for small and large IMF clock angle exist near or outside the specified high resolution grid region $-6<x<10,-10<y<10,-5<z<5 \mathrm{R}_{\mathrm{E}}$. We test the dependence of the nulls and separators on grid resolution by performing additional simulations with $\theta_{\mathrm{IMF}}=60^{\circ}, 150^{\circ}$, and $165^{\circ}$. The same base simulation parameters described in Chapter 2 are used, except the $0.125 \mathrm{R}_{\mathrm{E}}$ resolution region with $\theta_{\mathrm{IMF}}=60^{\circ}$ spans $-6<x<$ $10,-10<y<10,-12<z<12 \mathrm{R}_{\mathrm{E}}$ and $-6<x<10,-15<y<15,-10<z<10 \mathrm{R}_{\mathrm{E}}$ for the two southward IMF simulations.

The nulls in the higher resolution simulations are located at $\mathbf{r}_{\mathrm{Null}}=(-0.19, \pm 5.23$, $\pm 9.63) \mathrm{R}_{\mathrm{E}}$ for $60^{\circ}, \mathbf{r}_{\mathrm{Null}}=(-1.58, \pm 11.12, \pm 3.20) \mathrm{R}_{\mathrm{E}}$ for $150^{\circ}$, and $\mathbf{r}_{\mathrm{Null}}=(0.30, \pm 10.62$ $\pm 2.12) \mathrm{R}_{\mathrm{E}}$ for $165^{\circ}$. The nulls in the higher resolution simulations are $\simeq 3$ high resolution grid cells away from their lower resolution counterparts for $60^{\circ}, \simeq 5$ high resolution grid cells for $150^{\circ}$, and $\simeq 7$ high resolution grid cells for $165^{\circ}$. The location of the subsolar point (as measured by the last closed field line on the $x$-axis) is $x=8.62 \mathrm{R}_{\mathrm{E}}$ in the $60^{\circ}$ higher resolution simulation, compared to $x=8.44 \mathrm{R}_{\mathrm{E}}$ for the lower resolution simulation, a difference of about 1.5 grid cells; the last closed field lines for $150^{\circ}$ and $165^{\circ}$ are within a grid cell of their lower resolution counterparts. We trace separators in all higher resolution simulations and find that the separators in the higher resolution simulations do not deviate significantly from the lower resolution separators (not shown). This motivates that the resolution is sufficient to obtain accurate null locations and separators. 


\subsection{Summary}

The present study has focused on the dayside magnetopause, but it could be useful in other contexts. Xiao et al. $(2006,2007)$ observed magnetic nulls and separators at Earth's nightside and it is plausible that the method described here could locate separators at the nightside. The method described in the present study could also be used in studies of the solar corona and other planetary magnetospheres.

The technique presented here has been implemented by other researchers studying magnetic reconnection at the dayside magnetopause and elsewhere. For example, it was used to identify separators in three-dimensional particle-in-cell simulations of reconnection in a slab geometry (Olson et al., 2013). The technique is also being adapted to identify reconnection in global magnetospheric simulations with the Hall term, another non-ideal MHD

effect (K. Maynard, GEM Summer Workshop, 2014) Also, a recent study has increased the algorithm's efficiency, compared it to other techniques, and generalized it to trace separators in the presence of FTEs (Glocer et al., 2015). 


\section{Chapter 4}

\section{Comparative Analysis of Dayside}

\section{Magnetic Reconnection Models}

With the technique described in Chapter 3, we can identify locations where reconnection can occur. We proceed to test the predictions of different dayside reconnection models to determine how well each predicts the separator in our simulations.

The location of dayside magnetic reconnection for oblique IMF was originally discussed in terms of being anti-parallel (Crooker, 1979), where the claim was made that reconnection only happens at locations where the magnetosheath and magnetospheric magnetic fields were perfectly anti-parallel. Conversely, others argued that reconnection can occur between oblique magnetic fields so long as some component of the two magnetic fields is oppositely directed. The resulting "component reconnection" hypothesis argued that reconnection is constrained to occur with a fixed orientation where the out-of-plane (guide) magnetic field is uniform (Sonnerup, 1974; Gonzalez and Mozer, 1974). A number of literature reviews have been written on this subject (Cowley, 1976; Dorelli et al., 2007; Paschmann, 2008; 
Fuselier et al., 2011; Guo et al., 2013); the upshot is that both component and anti-parallel reconnection have been seen in observations and simulations. This implies that neither model can explain all the data, so models going beyond them are necessary.

Some recent models, introduced here but discussed in further detail in Section 4.1, suggest that reconnection occurs where some parameter is maximized which produces reconnection at an optimum efficiency. One such model is the maximum magnetic shear model (Trattner et al., 2007), an extension of the anti-parallel reconnection hypothesis. This model suggests that reconnection occurs where the magnetic shear angle between the magnetospheric and magnetosheath magnetic fields is locally a maximum. Three related models suggest reconnection occurs where the reconnection outflow speed is maximized (Swisdak and Drake, 2007), the reconnection rate is maximized [Shay, private communication, 2009; Borovsky (2013)], and the reconnecting field's magnetic energy is maximized (Hesse et al., 2013). Other models suggest that reconnection occurs where the current density is a maximum (Alexeev et al., 1998) or where the magnetospheric magnetic field and the draped IMF are bisected (Moore et al., 2002).

There are a number of studies testing some of these models in different contexts. The maximum magnetic shear model was shown to be consistent with observations of magnetic reconnection at planetary magnetopauses with data from Cluster (Trattner et al., 2007; Dunlop et al., 2011), THEMIS (Trattner et al., 2012), and Cassini (Fuselier et al., 2014). Also, observations (Teh and Sonnerup, 2008) and simulations (Schreier et al., 2010) suggest that the maximization of the asymmetric reconnection outflow speed controls the location and orientation of reconnection, although it is difficult to distinguish maximum outflow speed from maximum reconnection rate. The angle of bisection hypothesis has been tested with observations and in global magnetospheric simulations (Pulkkinen et al., 2010) and in 
two-dimensional (2D) reconnection simulations (Hesse et al., 2013).

Consequently, a careful assessment of the correctness of the recent models for the location and orientation of reconnection begins with a comparison of their predictions to the measured magnetic separators. To do so, we interpret the models, as Trattner et al. (2007) has done with the maximum magnetic shear model, as predicting the collections of points that locally maximize a particular quantity rather than the single point that globally maximizes the quantity in question.

The goal is to systematically test the recent models against the separators arising in self-consistent global simulations of the magnetosphere. To do so, we employ image processing techniques to unambiguously determine each model's prediction in our simulations. Each model's prediction is then compared with the magnetic separator determined with the algorithm described in Chapter 3. We test each model's agreement with the separators for various IMF clock angle $\theta_{\mathrm{IMF}}$, IMF strength $B_{\mathrm{IMF}}$, and dipole tilt angle. We also present a test of one possible way to incorporate the effects of a flow shear parallel to the reconnecting fields into the models based on reconnection outflow speed and reconnection rate, although recent results of Doss et al. (in prep.) suggest this formulation is incorrect.

The layout of this chapter is as follows: Section 4.1 reviews the models of dayside reconnection location that we test in the present study. Section 4.2 describes our methodology: the method used to determine the magnetopause, a robust image processing algorithm to determine each model's prediction, and details of our global magnetospheric simulations. Our results are presented in Section 4.3. A brief summary of our results are discussed in Section 4.4 . 


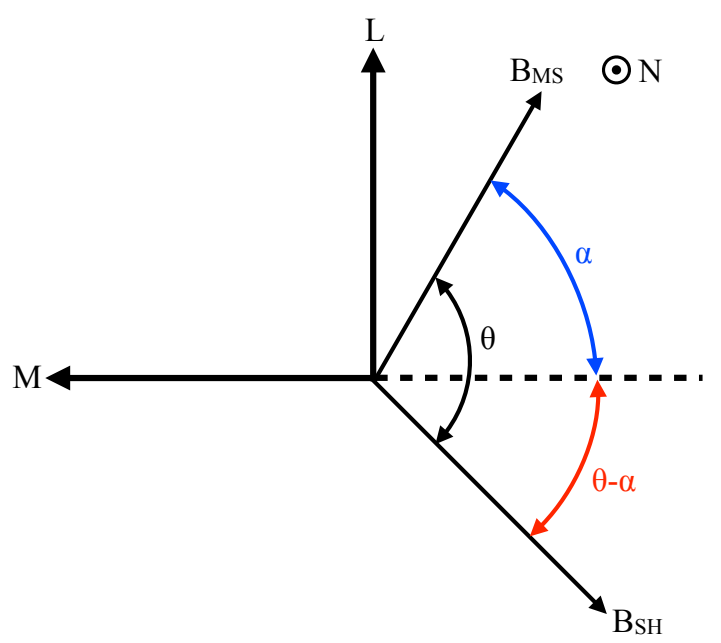

Figure 4.1: Schematic of an arbitrary magnetospheric magnetic field $\mathbf{B}_{\mathrm{MS}}$ making an angle $\theta$ with the magnetosheath magnetic field $\mathbf{B}_{\mathrm{SH}}$ in the boundary normal (LMN) coordinate system. The angle $\alpha$ is the angle the magnetospheric field makes with the $M$ axis. Adapted from Sonnerup (1974) and Swisdak and Drake (2007).

\subsection{Reconnection Location Models}

This section reviews the models tested in this study. We employ the boundary normal (LMN) coordinate system. The $N$ direction is the magnetopause normal corresponding to the inflow direction, the $L$ direction corresponds to the reconnecting component of the magnetic field, and the $M$ direction gives the direction of the out-of-plane (guide) magnetic field, completing the right-handed triplet. Figure 4.1, adapted from Sonnerup (1974) and Swisdak and Drake (2007), displays the magnetospheric and magnetosheath magnetic fields $\mathbf{B}_{\mathrm{MS}}$ and $\mathbf{B}_{\mathrm{SH}}$ at the magnetopause viewed along the inflow direction. These two fields point in arbitrary directions in the $L-M$ plane, making a magnetic shear angle of $\theta$ with each other. The angle $\alpha$ is the angle $\mathbf{B}_{\mathrm{MS}}$ makes with the $M$ axis and is constrained to occur within the range $0<\alpha<\theta$. It is tacitly assumed that the magnetic field components along the $N$ axis are weak.

In the maximum magnetic shear model (Trattner et al., 2007), reconnection occurs at magnetopause locations where the magnetic shear angle $\theta$ between the magnetospheric and 
magnetosheath magnetic fields is a maximum. This model identifies the anti-parallel reconnection locations of Crooker (1979) with high fidelity, while also identifying additional locations away from the anti-parallel regions where $\theta$ is locally a maximum. The magnetic shear angle $\theta$ is calculated from the magnetospheric and magnetosheath magnetic fields as

$$
\cos \theta=\frac{\mathbf{B}_{\mathrm{MS}} \cdot \mathbf{B}_{\mathrm{SH}}}{B_{\mathrm{MS}} B_{\mathrm{SH}}} .
$$

Alternative explanations have suggested that reconnection occurs at locations where some measure of reconnection efficiency is maximized. In the model of Swisdak and Drake (2007), reconnection on the dayside is oriented (has an angle $\alpha$ ) to maximize the reconnection outflow speed. In a subsequent model, reconnection orients so as to maximize the reconnection electric field $E$ [M. A. Shay, private communication, 2009; Borovsky (2013)]. To calculate the predictions from these models at the dayside magnetopause, one needs the plasma parameters of the magnetosheath and magnetosphere, where asymmetries in the magnetic field and plasma density strongly affect reconnection. The scaling of asymmetric reconnection was developed for 2D antiparallel reconnection using conservation of mass and energy (Cassak and Shay, 2007); results for the asymmetric outflow speed and reconnection rates are given in Eqs. (1.20)-(1.23).

We follow the example of Swisdak and Drake (2007) and modify these expressions for the LMN coordinate system, where the reconnecting component of the upstream magnetospheric magnetic field can be written as

$$
B_{\mathrm{MS}, L}=B_{\mathrm{MS}} \sin \alpha
$$


and for the magnetosheath as

$$
B_{\mathrm{SH}, L}=B_{\mathrm{SH}} \sin (\theta-\alpha)
$$

as depicted in Fig. 4.1. We substitute these $L$ components of the magnetic field for the anti-parallel fields $B_{1}$ and $B_{2}$ in Eqs. (1.21)-(1.23) giving, in LMN coordinates, the hybrid Alfvèn speed

$$
c_{A, \text { out }}^{2} \sim \frac{B_{\mathrm{MS}, L} B_{\mathrm{SH}, L}\left(B_{\mathrm{MS}, L}+B_{\mathrm{SH}, L}\right)}{\mu_{0}\left(\rho_{\mathrm{MS}} B_{\mathrm{SH}, L}+\rho_{\mathrm{SH}} B_{\mathrm{MS}, L}\right)}
$$

the asymmetric electric field

$$
E \sim c_{A, \text { out }} \frac{B_{\mathrm{MS}, L} B_{\mathrm{SH}, L}}{B_{\mathrm{MS}, L}+B_{\mathrm{SH}, L}} \frac{2 \delta}{L}
$$

and the Sweet-Parker collisional reconnection rate

$$
E_{S P} \sim \sqrt{\frac{\eta c_{A, \text { out }}}{\mu_{0} L} B_{\mathrm{MS}, L} B_{\mathrm{SH}, L}}
$$

where $\rho_{\mathrm{MS}}$ and $\rho_{\mathrm{SH}}$ are the upstream magnetospheric and magnetosheath plasma mass densities, respectively, $\mu_{0}$ is the permeability of free space, $\eta$ is the plasma resistivity, and $\delta$ and $L$ are the half-width and half-length of the diffusion region, respectively. The asymmetric outflow speed is maximized by setting

$$
\frac{\partial c_{A, \text { out }}^{2}}{\partial \alpha}=0
$$

and solving for $\alpha$ (Swisdak and Drake, 2007). A similar approach is used to maximize E [M. A. Shay, private communication, 2009; Borovsky (2013)]. In practice, we interpret 
these as saying that the local $c_{A, \text { out }}$ and $E$ can be found at every location on the magnetopause by finding $\alpha$ at every location, and that reconnection occurs where the parameter is a local maximum. We note in passing that this approach assumes that any nonzero $B_{M}$ component of either magnetic field does not affect the outflow speed or reconnection rate. This is not likely to be correct in the real magnetosphere where finite Larmor radius effects are important, but should be accurate for the MHD simulations in the present study. Another limitation of the present formulation of these arguments is that they neglect the importance of the plasma bulk flow parallel to the reconnecting magnetic field components; we detail an attempt to account for this effect and measure the impact on the predictions of the asymmetric reconnection models in Section 4.3.4.

More recently, Hesse et al. (2013) suggested that the reconnection rate is proportional to the product of the reconnecting component's magnetic energies,

$$
E \propto B_{\mathrm{MS}, L}^{2} B_{\mathrm{SH}, L}^{2},
$$

and reconnection maximizes this product. Applying a maximization argument with respect to $\alpha$ gives a solution of $\alpha=\theta / 2$; this is equivalent to previous arguments where the guide field bisects the merging magnetosheath and magnetospheric magnetic fields (Moore et al., 2002; Borovsky, 2008).

We also test the model that predicts reconnection to occur where the current density magnitude $|\mathbf{J}|$ is a maximum (Alexeev et al., 1998). A related approach has been to look at the divergence of the Poynting vector. Reconnection has been located by measuring this quantity in observations (Anekallu et al., 2013) and simulations (Papadopoulos et al., 1999; Palmroth et al., 2003; Laitinen et al., 2006, 2007; Pulkkinen et al., 2010; Palmroth et al., 
2012; Hoilijoki et al., 2014). In steady-state, the divergence of the Poynting vector is

$$
\nabla \cdot\left(\frac{\mathbf{E} \times \mathbf{B}}{\mu_{0}}\right) \sim-\mathbf{E} \cdot \mathbf{J}
$$

where $\mathbf{E}$ is the electric field given by Ohm's law. The present study employs a large explicit resistivity, as described in Chapter 2. In this system, the divergence of the Poynting vector simplifies to $-\eta J^{2}$ at the reconnection site. We therefore argue that, up to a scaling factor, the Poynting vector's divergence would give similar results as the maximum current density magnitude argument for this study.

For completeness, we also test the component reconnection hypothesis as originally proposed by Sonnerup (1974) and Gonzalez and Mozer (1974), even though observations (Gosling et al., 2007; Gosling, 2007; Teh and Sonnerup, 2008; Phan et al., 2009, 2010; Gosling and Phan, 2013; DiBraccio et al., 2013) and simulations (Schreier et al., 2010) suggest the model is not valid. In this model, the local reconnection geometry orients itself such that the $M$ component of the magnetic field is uniform, which, using the notation in Fig. 4.1, implies

$$
\tan \alpha=\frac{1 / b-\cos \theta}{\sin \theta}
$$

where $b=B_{\mathrm{SH}} / B_{\mathrm{MS}}$. This model places an additional constraint on the orientation angle $\alpha$, with valid solutions only when the magnetic shear angle $\theta$ is greater than the critical shear angle $\theta_{c}=\cos ^{-1}(1 / b)$. 


\subsection{Methodology}

Each of the models discussed in the previous section suggest reconnection at the dayside magnetopause occurs where some parameter is a local maximum. We model our approach after Trattner et al. (2007), who identified a curve corresponding to where the magnetic shear angle $\theta$ was a local maximum at the magnetopause. The magnetospheric magnetic field was modeled using the T96 Tsyganenko model, an empirical model encompassing almost 25 years of magnetospheric observations to model the magnetospheric magnetic field resulting from the solar wind's compression of the magnetopause and accounting for contributions to the magnetic field from magnetospheric currents (Tsyganenko, 1995). The Cooling model is used to parametrize the magnetosheath magnetic field, density and flow, which arises from the solar wind flow causing the IMF to drape over the magnetopause (Cooling et al., 2001). Using these magnetic field models, the authors calculate $\theta$, given by Eq. (4.1), at many locations on the dayside magnetopause. The local shear angle $\theta$ was plotted as a function of the magnetopause's $y$ and $z$ coordinates (it was projected into the $x=0$ plane) and maxima in this shear angle map were determined by finding maxima along cuts parallel to the IMF (K. Trattner, private communication, 2013).

The following sections detail how we robustly identify the magnetopause and calculate model predictions in our global magnetospheric simulations.

\subsubsection{Identification of the Magnetopause and Plasma Parameter Sampling}

To identify the magnetopause in our global magnetospheric simulations, we modify the method of Němeček et al. (2011). (One may alternatively adopt the method described 
in Palmroth et al. (2003) to identify the magnetopause using streamlines, although this is not tested here.) The current density magnitude is sampled radially from $7 \leq r<20 \mathrm{R}_{\mathrm{E}}$ at our highest grid cell resolution $\left[0.125 \mathrm{R}_{\mathrm{E}}\right]$. The location of a local maximum in the current density magnitude at smallest $r$ is identified as the magnetopause and this location's coordinates $\mathbf{r}_{\mathrm{MP}}$ are saved; the chosen sampling range excludes the ring current, but we take additional care by ensuring each point has a radial distance within $2 \mathrm{R}_{\mathrm{E}}$ of the previous point's radial distance. We employ spherical coordinates in the GSM system, where the azimuthal angle $\phi$ is measured from the $+x$ axis and the polar angle $\theta$ is measured from the $+z$ axis; we discretize both into $5^{\circ}$ increments. (Other increments were tested and found to converge to the $5^{\circ}$ results.) We use an azimuthal range of $-130^{\circ} \leq \phi \leq 130^{\circ}$ to map the magnetopause for northward IMF orientations $\left(\theta_{\mathrm{IMF}}=\left[30^{\circ}, 60^{\circ}, 90^{\circ}\right]\right.$ for the purposes of this study $)$ and $-110^{\circ} \leq \phi \leq 110^{\circ}$ for southward IMF orientations $\left(\theta_{\mathrm{IMF}}=\left[120^{\circ}, 150^{\circ}, 165^{\circ}\right]\right)$; the polar angle has a range $0^{\circ} \leq \theta \leq 180^{\circ}$ for both northward and southward IMF orientations.

An example of this process can be seen in Fig. 4.2, displaying the magnetopause surface as green spheres for a simulation with IMF clock angle of $120^{\circ}$ Additionally, the separator for this simulation is plotted in blue. It lies within the magnetopause surface, as it should, confirming this method. The grid is rotated out of the ecliptic plane; the spherical coordinate system is rotated counter-clockwise about the $+x$ axis by an angle $\theta_{\mathrm{IMF}} / 2$. This is done to achieve near uniform sampling near the separator and each model's prediction, i.e., near $\theta \approx 90^{\circ}$ in the rotated frame.

Once the approximate location of the magnetopause has been determined, the local plasma parameters of the magnetosphere and magnetosheath are sampled. The appropriate way to do this is to sample along the magnetopause normal $\hat{\mathbf{n}}$ at each magnetopause position $\mathbf{r}_{\mathrm{MP}}$. One may perform a minimum variance analysis (MVA) on the magnetic field (Sonnerup 


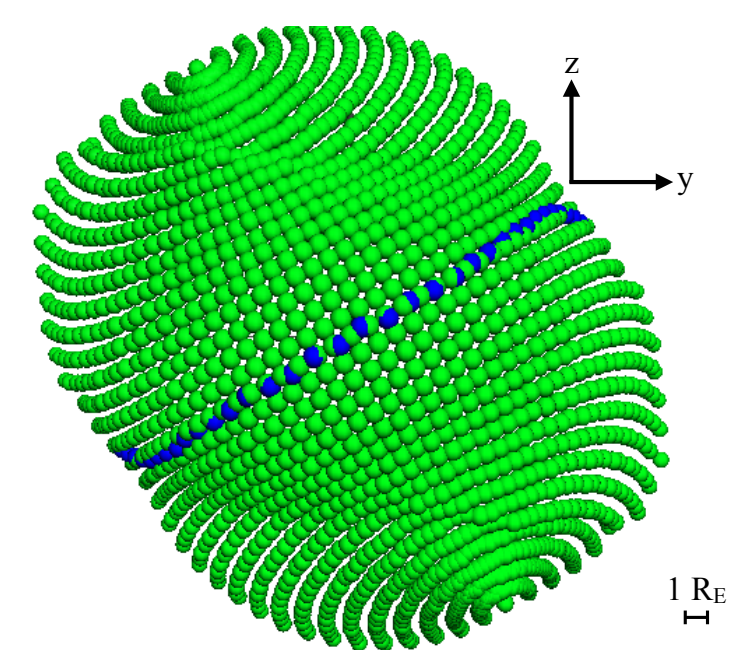

Figure 4.2: Magnetopause locations (green) from maxima in the current density's magnitude in a simulation with $\theta_{\mathrm{IMF}}=120^{\circ}$. The magnetic separator (blue) is shown for reference.

and Cahill, 1967) to determine the magnetopause normal. MVA is a statistical technique that compares the variations among all three magnetic field components in order to determine the axis along which the magnetic field varies the least. However, we find in practice this results in incorrect magnetopause normals since the magnetospheric and magnetosheath magnetic fields are parallel near the separator, violating the core assumption of MVA.

Instead, the normal is calculated with the method described in Hoppe et al. (1992). The algorithm is to take a single point on the magnetopause $\mathbf{r}_{\mathrm{MP}}$ and find its four nearest neighbors $\mathbf{r}_{k}$ on the magnetopause, with $k \in[1,2,3,4]$. Calculate the difference vector $\mathbf{r}_{k}-\mathbf{r}_{\mathrm{MP}}$, which gives the distance from the point on the magnetopause and its $k$ th nearest neighbor. Then, construct the co-variant matrix $\mathbf{M}$, whose $i j$ element is

$$
M_{i j}\left(\mathbf{r}_{\mathrm{MP}}\right)=\sum_{k}\left(\mathbf{r}_{k}-\mathbf{r}_{\mathrm{MP}}\right)_{i}\left(\mathbf{r}_{k}-\mathbf{r}_{\mathrm{MP}}\right)_{j}
$$

where $i$ and $j$ refer to the $x, y$, or $z$ component in GSM coordinates. The eigenvector corresponding to the minimum eigenvalue of $\mathbf{M}$ is the magnetopause normal; this normal 
direction is chosen to point away from Earth, i.e., $\hat{\mathbf{x}} \cdot \hat{\mathbf{n}} \geq 0$, and has been confirmed by inspection. This procedure has a physical interpretation of placing several planes at $\mathbf{r}_{\mathrm{MP}}$ with arbitrary orientations; the normal of the plane that minimizes the distance between the plane and the nearest neighbors is the magnetopause's normal.

The plasma parameters are sampled along the normal vector $\hat{\mathbf{n}}$ at our highest resolution $\left[0.125 \mathrm{R}_{\mathrm{E}}\right]$ between $\mathbf{r}_{\mathrm{MP}}-\left(5 \mathrm{R}_{\mathrm{E}}\right) \hat{\mathbf{n}}$ and $\mathbf{r}_{\mathrm{MP}}+\left(5 \mathrm{R}_{\mathrm{E}}\right) \hat{\mathbf{n}}$. To accurately measure the asymptotic plasma densities and magnetic fields undergoing magnetic reconnection, we employ the method used by Cassak and Shay (2009) for systems with asymmetries in either parameter. The current density is measured along $\hat{\mathbf{n}}$ to determine where it falls to $1 / e$ of its maximum value in the magnetosheath. The distance between the current maximum and this location is the current sheet's half-thickness $\delta$. The upstream plasma parameters of the magnetosphere are measured at $\mathbf{r}_{\mathrm{MP}}-(2 \delta) \hat{\mathbf{n}}$ and those of the magnetosheath at $\mathbf{r}_{\mathrm{MP}}+(2 \delta) \hat{\mathbf{n}}$. We confirm the measured magnetospheric and magnetosheath magnetic fields and densities are representative of the asymptotic values, as desired. The raw upstream magnetic fields can have small normal components $\left[\left|B_{n}\right| \sim 1 \mathrm{nT}\right]$; these are removed prior to calculations. These values are used as $\mathbf{B}_{\mathrm{MS}}, \mathbf{B}_{\mathrm{SH}}, \rho_{M S}$, and $\rho_{S H}$ for the models discussed in Section 4.1.

We note in passing that identifying the magnetopause as the maximum in the current density's magnitude has its limitations. One consequence of asymmetric reconnection systems is that the stagnation point separates from the reconnection X-line in 2D reconnection (Cassak and Shay, 2007); the maximum of the out-of-plane current density also separates from the X-line. We will describe in Section 4.3 .2 a test of reconnection location models in distinct simulations with IMF strengths of 20,5 , and $2 \mathrm{nT}$. Decreasing $B_{\mathrm{IMF}}$ in this manner results in the current density located on the magnetospheric side of the separator, with distances of $0.07[20 \mathrm{nT}], 0.36[5 \mathrm{nT}]$, and $0.51 \mathrm{R}_{\mathrm{E}}[2 \mathrm{nT}]$; we note that the separation distances are 
resolved in the 5 and $2 \mathrm{nT}$ simulations since our maximum resolution is $0.125 \mathrm{R}_{\mathrm{E}}$.

However, since we keep the solar wind speed the same for all these simulations, the magnetopause should occupy the same location regardless of $B_{\mathrm{IMF}}$. We motivate why this should be the case using the MHD momentum equation, Eq. (2.3). For our base simulation parameters, the solar wind's magnetic pressure $B_{\mathrm{IMF}}^{2} /\left(2 \mu_{0}\right) \approx 0.3 \mathrm{nPa}$ is approximately an order of magnitude smaller than the solar wind ram pressure $p_{R a m}=\rho u_{x}^{2} / 2 \approx 2.7 \mathrm{nPa}$. (The thermal pressure has a negligible contribution with $p \sim 0.01 p_{\text {Ram }}$.) The location of the magnetopause is determined by pressure balance between the magnetosphere's magnetic pressure and the solar wind's total pressure. Therefore, lowering the IMF strength should have negligible effects on the magnetopause's location, and at worst would move one simulation grid cell $(\Delta=0.125)$ outward along the magnetopause normal. Consequently, the change in the location of the current maximum is not due to the magnetopause moving, but rather the structure of the current between asymmetric magnetic fields. Fortunately, for the purposes of the present study, this effect is not critical because we use the magnetopause to get the upstream plasma parameters, and our sampling method ensures that the magnetospheric and magnetosheath values are measured far enough upstream of the magnetopause to get appropriate representative values.

\subsubsection{Finding Model Predictions Using Image Processing Tech- niques}

Having found the approximate location of the magnetopause and the parameters locally governing reconnection $\left(\rho_{\mathrm{MS}}, \rho_{\mathrm{SH}}, \mathbf{B}_{\mathrm{MS}}, \mathbf{B}_{\mathrm{SH}}\right)$, the relevant quantities for each model are calculated at every point on the magnetopause. The magnetic shear angle $\theta$ is calculated 
from Eq. (4.1) at every point and is retained. Then at every point, $\alpha$ is discretized in the range $0<\alpha<\theta$. For each value of $\alpha, B_{\mathrm{MS}, L}$ and $B_{\mathrm{SH}, L}$ are calculated from Eqs. (4.2) and (4.3), and $c_{A, \text { out }}, E_{S P}$, and $B_{\mathrm{MS}, L}^{2} B_{S H, L}^{2}$ are calculated for that $\alpha$. The $\alpha$ which maximizes the quantity in question is retained, and the value of $c_{A, \text { out }}, E_{S P}$, and $B_{\mathrm{MS}, L}^{2} B_{S H, L}^{2}$ is retained for that point. (One could alternately determine $\alpha$ from $\partial c_{A, \text { out }} / \partial \alpha=0$ and $\partial E / \partial \alpha=0$.) The magnetopause current density magnitude is also retained at every point.

The results for each model are projected into the $x=0$ plane, resulting in a $2 \mathrm{D}$ image $\mathscr{I}(y, z)$, where $\mathscr{I}$ represents the quantity in question for each model. An example is shown in Fig. 4.3 for the maximum magnetic shear model, where $\mathscr{I}$ corresponds to $\theta$, which is plotted as the third dimension in blue spheres for the $\theta_{\mathrm{IMF}}=120^{\circ}$ simulation. The magnetic shear angle surface has a saddle shape, as do the surfaces for all the other models tested (not shown). This plot makes it clear that finding the collection of locations where the quantity in question is locally a maximum is tantamount to finding the ridge of local maxima in $\mathscr{I}$.

We achieve this in a robust way using image processing techniques using a ridge finding algorithm (Lindeberg, 1993, 1998). First, we construct the Hessian tensor $H_{i j}(y, z)=$ $\partial^{2} \mathscr{I}(y, z) / \partial x_{i} \partial x_{j}$ via finite differencing at every point in the image. Diagonalizing the Hessian matrix defines a $(p, q)$ coordinate system, where the eigenvector associated with the maximum absolute eigenvalue points in the direction of the local maxima. (Without loss of generality, we designate that eigenvector as $\hat{\mathbf{p}}$, the unit vector for the $p$ direction.) Local maxima are determined by finding all locations where $\partial \mathscr{I} / \partial p=0$ (local extrema test) and $\partial^{2} \mathscr{I} / \partial p^{2}<0$ (local maxima test) using linear interpolation between nearest neighbors ( Lindeberg, 1993).

The results of this technique for the maximum magnetic shear model for $\theta_{\mathrm{IMF}}=120^{\circ}$ are shown as the red spheres in Fig. 4.3. These red spheres clearly mark the ridge of the magnetic 


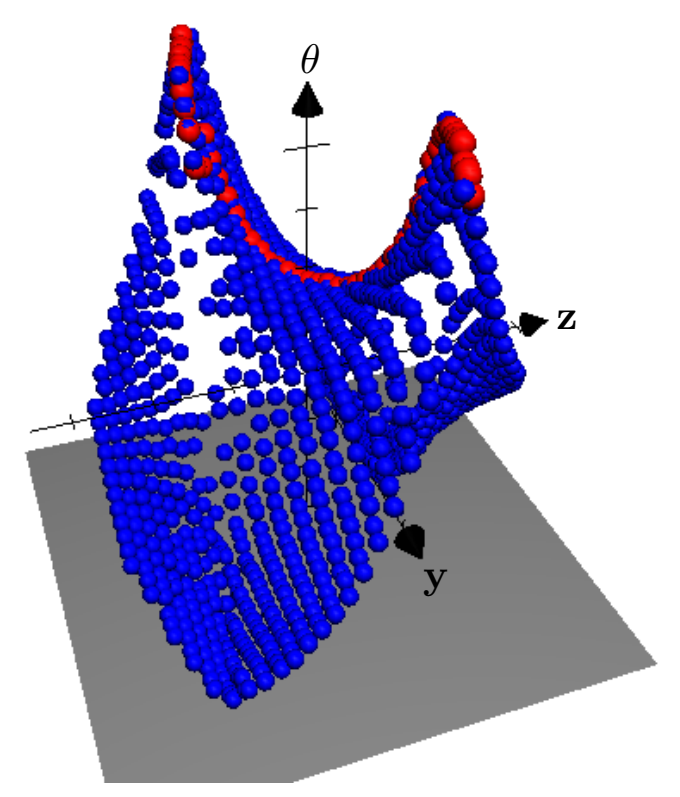

Figure 4.3: Magnetic shear angle $\theta$ (blue spheres) as a function of the magnetopause's $y$ and $z$ coordinates and the corresponding ridge of maximum magnetic shear angle (red spheres) in a simulation with $\theta_{\mathrm{IMF}}=120^{\circ}$.

shear surface as desired. The methodology we employ yields qualitatively similar results to previous studies testing the maximum magnetic shear model for comparable magnetospheric and solar wind conditions [see Fig. 1 in Fuselier et al. (2011)], indicating this is an appropriate technique to determine each model's prediction. A more thorough comparison between these techniques will be presented in Section 4.3.1.

\subsubsection{Determining Magnetic Separators}

We employ the separator mapping algorithm presented in Chapter 3 which reliably traces the dayside portion of the magnetic separators connecting the magnetic nulls in global magnetosphere simulations for any IMF direction. When the IMF has a northward component, observations have shown that reconnection occurs poleward of the magnetic cusps [see Wilder et al. (2014), and references therein]. We therefore trace portions of the magnetic separator $10 \mathrm{R}_{\mathrm{E}}$ nightward of the nulls in our simulations with $\theta_{\mathrm{IMF}}=30^{\circ}, 60^{\circ}$, and $90^{\circ}$. 

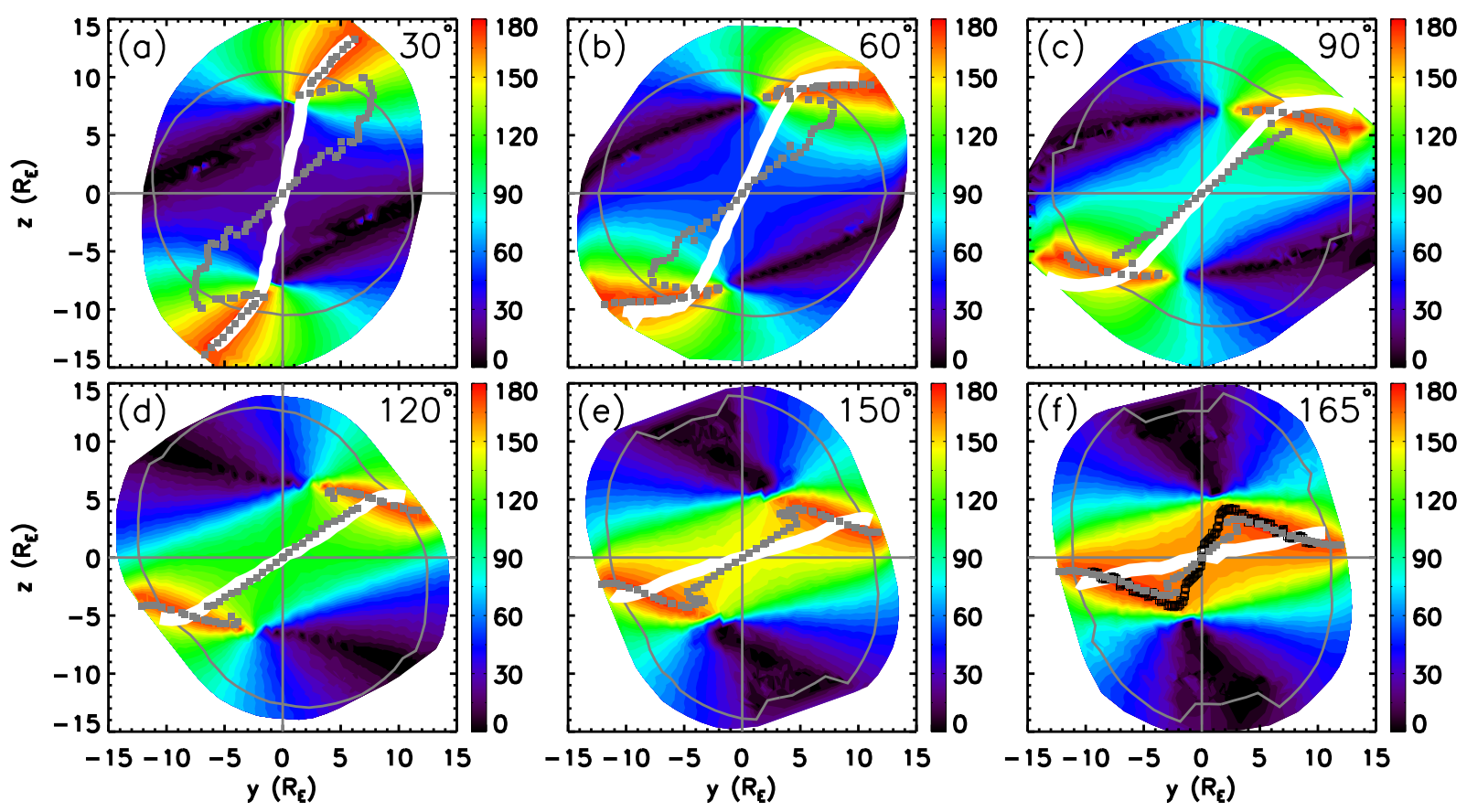

Figure 4.4: Results for the maximum magnetic shear model in simulations with $\theta_{\text {IMF }}$ of (a) $30^{\circ}$, (b) $60^{\circ}$, (c) $90^{\circ}$, (d) $120^{\circ}$, (e) $150^{\circ}$, and (f) $165^{\circ}$. The calculated magnetic shear angle $\theta$ is the color background, the gray squares display the curve of maximum magnetic shear angle, and the magnetic separator is displayed in white. The gray oval displays the magnetopause's projection in the $x=0$ plane. Maxima determined from cuts along the IMF direction (Trattner et al., 2007) are displayed as black squares in panel (f).

\subsection{Results}

We present results for the predicted locations for the models discussed in Section 4.1 and compare the results with the magnetic separators. Figures 4.4-4.8 display the results in a common format. The quantity of interest is displayed as the background color, the model's prediction is displayed with solid gray squares, and the magnetic separators determined with the algorithm described in Chapter 3 are displayed as the solid white line. The magnetopause's intersection with the $x=0$ plane is displayed as the gray oval. The panels for each figure correspond to IMF clock angles $\theta_{\mathrm{IMF}}$ of (a) $30^{\circ}$, (b) $60^{\circ}$, (c) $90^{\circ}$, (d) $120^{\circ}$, (e) $150^{\circ}$, and (f) $165^{\circ}$. Figure 4.4 displays the magnetic shear angle $\theta$ in degrees calculated from Eq. (4.1), Fig. 4.5 has the asymmetric outflow speed $c_{A, \text { out }}$ in $\mathrm{km} / \mathrm{s}$ calculated from Eq. (4.4), Fig. 4.6 

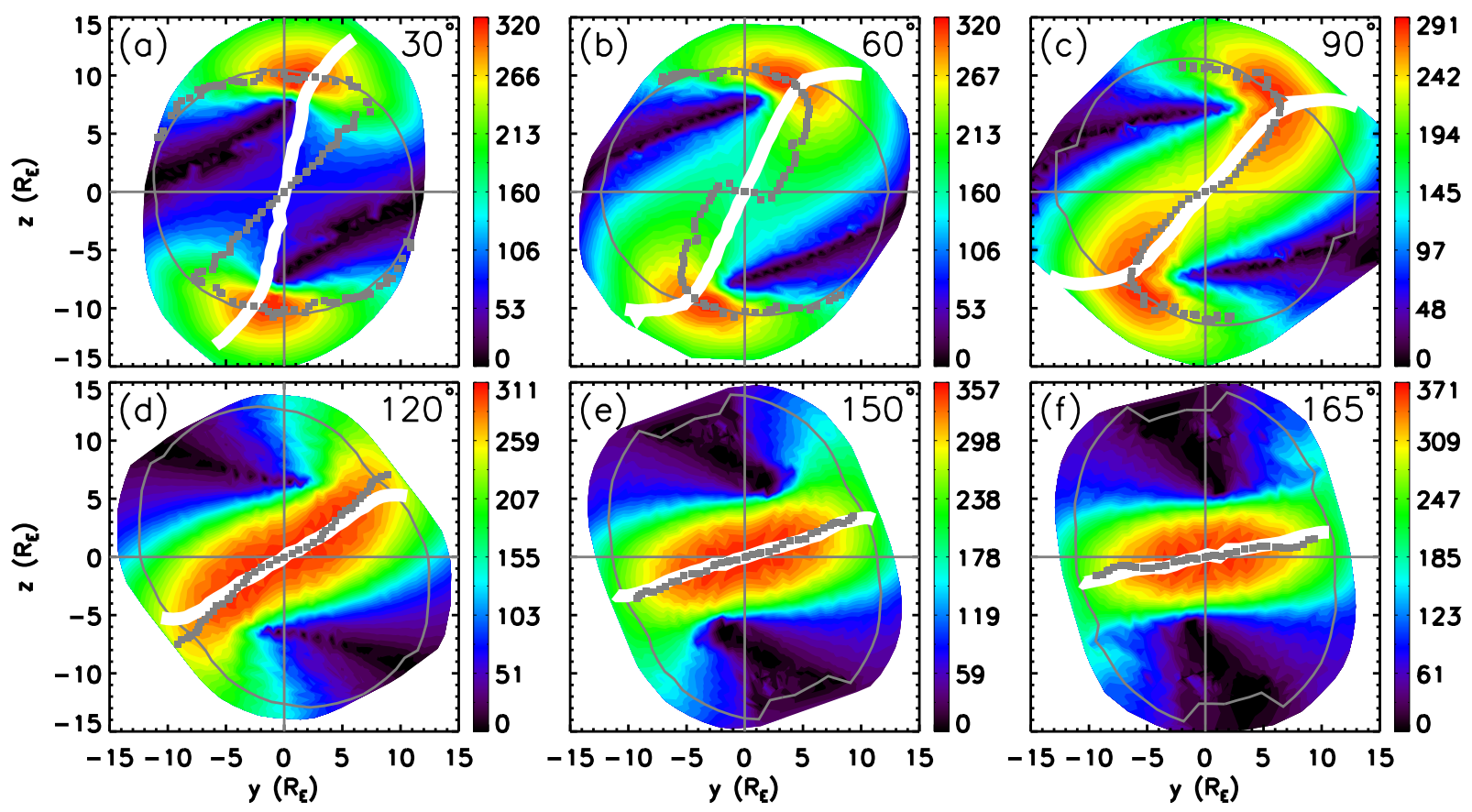

Figure 4.5: Results for the asymmetric outflow speed $c_{A, \text { out }}$. See the caption of Figure 4.4 for definitions.

has the asymmetric Sweet-Parker reconnection rate $E_{S P}$ in $\mathrm{mV} / \mathrm{m}$ calculated from Eq. (4.6), Fig. 4.7 has the quantity $B_{\mathrm{MS}, L}^{2} B_{\mathrm{SH}, L}^{2}$ from Eq. (4.7) in $10^{8} \mathrm{nT}^{4}$, and Figure 4.8 has the current density magnitude $|\mathbf{J}|$ in $\mathrm{nA} / \mathrm{m}^{2}$.

Figure 4.9 displays the results for the uniform $B_{M}$ component model. Plots (a) through (f) are for the same IMF clock angle simulations as previously discussed, with a few notable differences. The local $B_{M}$ component (guide field) is calculated by determining the orientation angle $\alpha$ from Eq. (4.8), and rotating about the determined boundary normal $\hat{\mathbf{n}}$. The gray arrows in Fig. 4.9 display the projection of the magnetic field's $M$ component in the $x=0$ plane; locations without any vector displayed are where reconnection is predicted to be geometrically impossible. The magnetic separator is displayed as the solid black line, with the magnetopause's location in the $x=0$ plane plotted in gray as before.

Section 4.3.1 discusses how the predictions of each model change with IMF clock angle for fixed solar wind conditions. We test the dependence on IMF strength of various models 

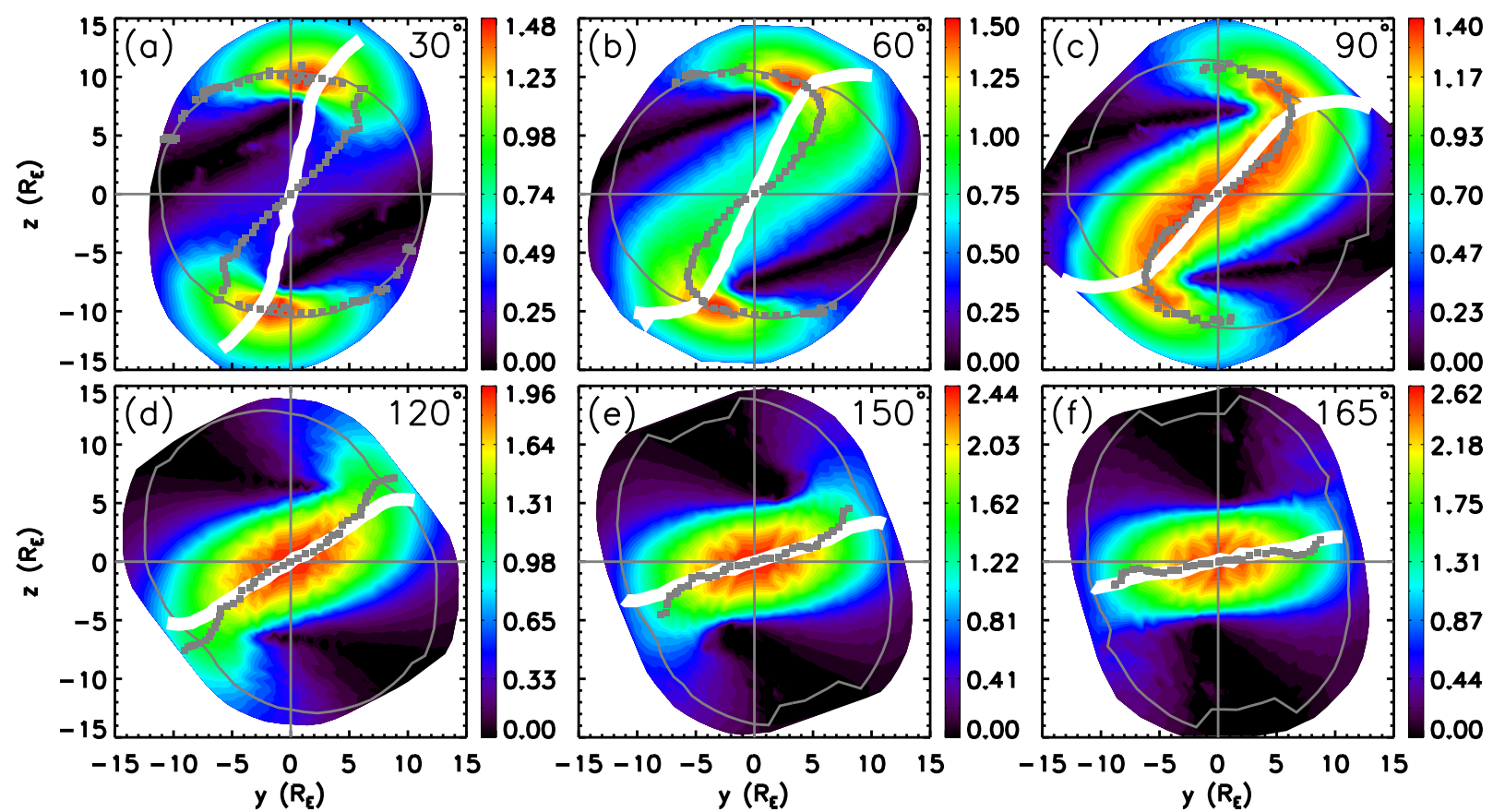

Figure 4.6: Results for the asymmetric Sweet-Parker reconnection rate $E_{S P}$. See the caption of Figure 4.4 for definitions.

in Section 4.3.2. Section 4.3.3 describes results from two distinct simulations that include a dipole tilt with IMF clock angles $120^{\circ}$ and $30^{\circ}$. Lastly, Section 4.3 .4 discusses an attempt to incorporate the effects of a plasma flow shear on reconnection into the outflow speed and reconnection rate models from Section 4.1.

\subsubsection{Clock Angle Dependence}

Before assessing each model, we perform a more careful comparison between our ridge finding technique and the one in Trattner et al. (2007). We plot the result using the technique of Trattner et al. (2007), which finds maxima of magnetic shear angle along cuts parallel to the IMF direction, as black squares in Fig. $4.4(\mathrm{f})$ for the $\theta_{\mathrm{IMF}}=165^{\circ}$ simulation. While qualitatively similar, there are apparent differences. If one takes cuts along different directions (not shown), the detected locations change, and can move to locations on the other side of 

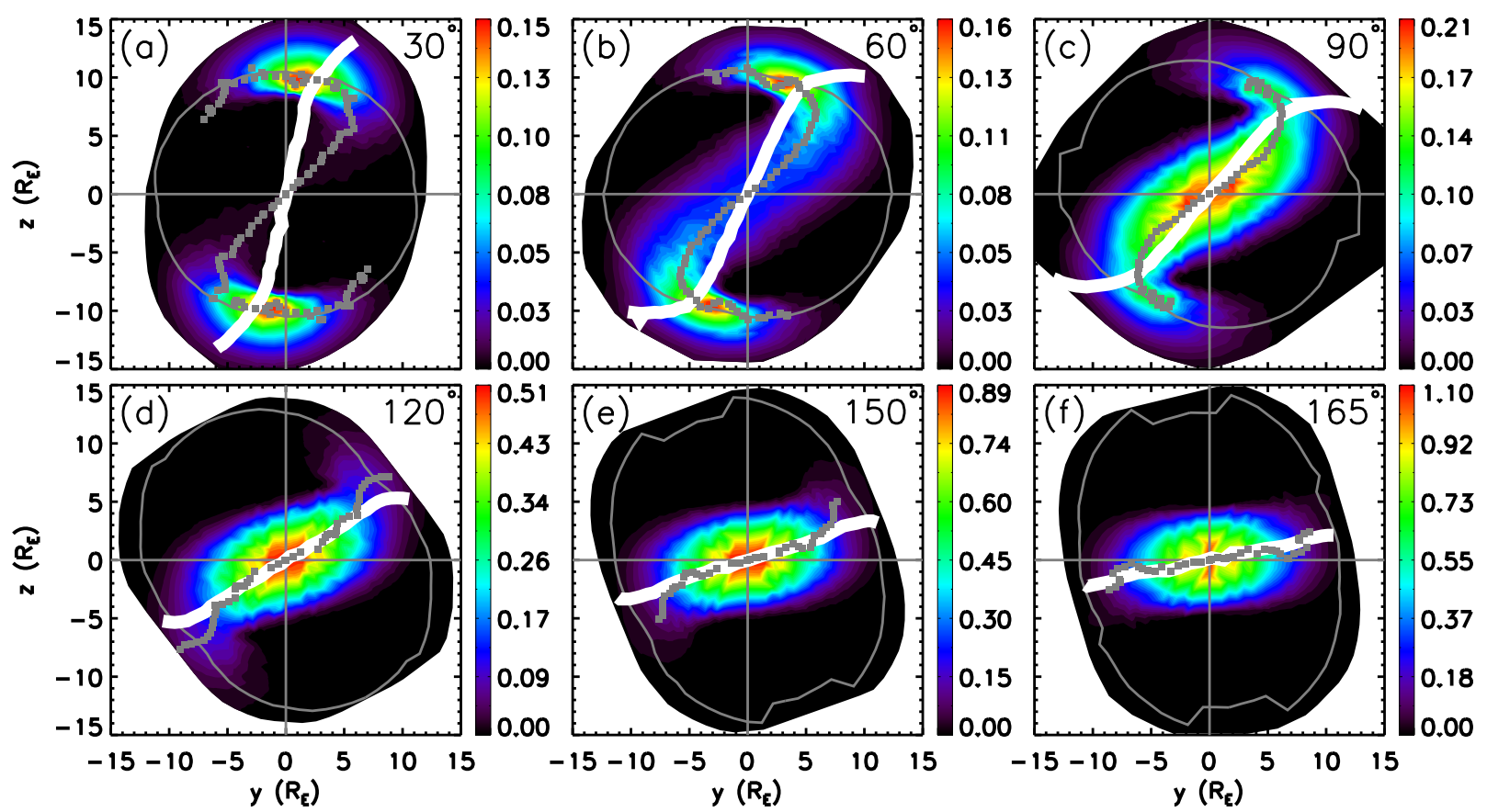

Figure 4.7: Results for the angle of bisection. See the caption of Figure 4.4 for definitions.

the image processing ridge. This underscores two points: (1) it is important to employ a user-independent technique, such as image processing, to locate each models' prediction as the Trattner et al. (2007) algorithm exhibits sensitivity to the cut orientation and (2) the noticeable changes in the prediction are caused by the relative flatness in the saddle region that occurs near the subsolar point in these results. Consequently, there are uncertainties that could cause minor changes to the predictions which should be kept in mind in the interpretation of these results.

We now focus on the predictions of each model and how they compare with the separators for all IMF clock angles. Of the models tested, the maximum magnetic shear as shown has the largest discrepancy with the dayside portion of the magnetic separators in simulations without a dipole tilt, but could have better agreement in light of the uncertainties discussed above. More importantly, only the maximum magnetic shear model reproduces the portion of the magnetic separator nightward of the magnetic nulls with high fidelity. These portions 

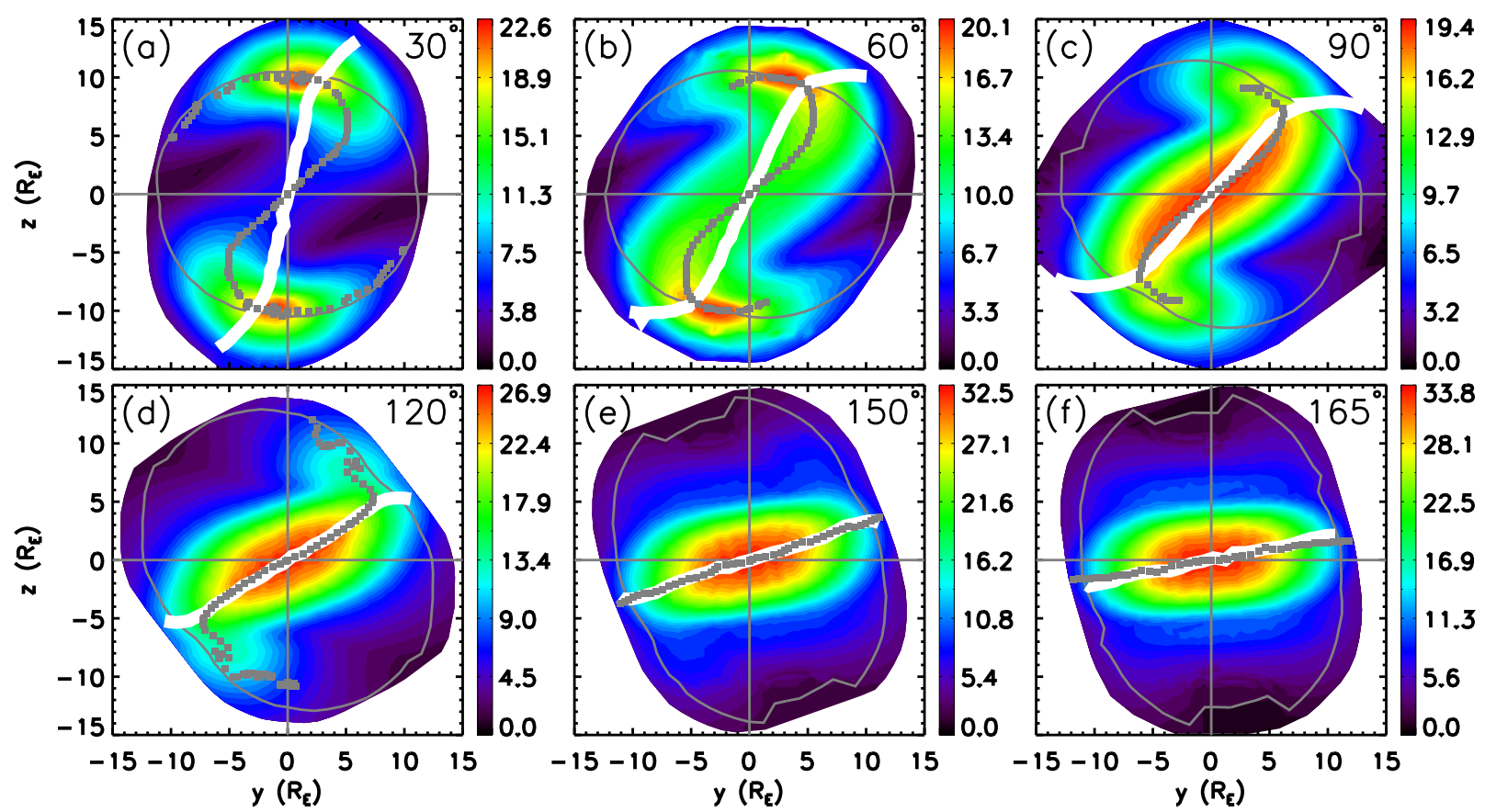

Figure 4.8: Results for the current density magnitude $|\mathbf{J}|$. See the caption of Figure 4.4 for definitions.

of the magnetic separators coincide with the antiparallel regions and are near the magnetic cusps, consistent with early expectations (Dungey, 1961, 1963; Crooker, 1979) and with observations [see Wilder et al. (2014), and references therein].

The asymmetric reconnection arguments $c_{A, \text { out }}$ and $E_{S P}$ show better agreement with the magnetic separators overall for the simulations with southward IMF orientations. The ridges of these models rotate around the $x$ axis, as does the separator, although there are differences between the separators and the model predictions for clock angles $\theta_{\mathrm{IMF}} \leq 120^{\circ}$.

The predictions for the angle of bisection and the maximum current density models have a similar shape as the other models' predictions through the subsolar point. Figures 4.7(a)(c) and 4.8(a)-(c) show intersections between the model predictions and magnetic separators near the antiparallel regions and at the subsolar point for $\theta_{\mathrm{IMF}} \leq 90^{\circ}$. The predictions of these arguments do follow the separator more closely for southward IMF orientations, and do not rotate around the magnetopause for northward orientations. 

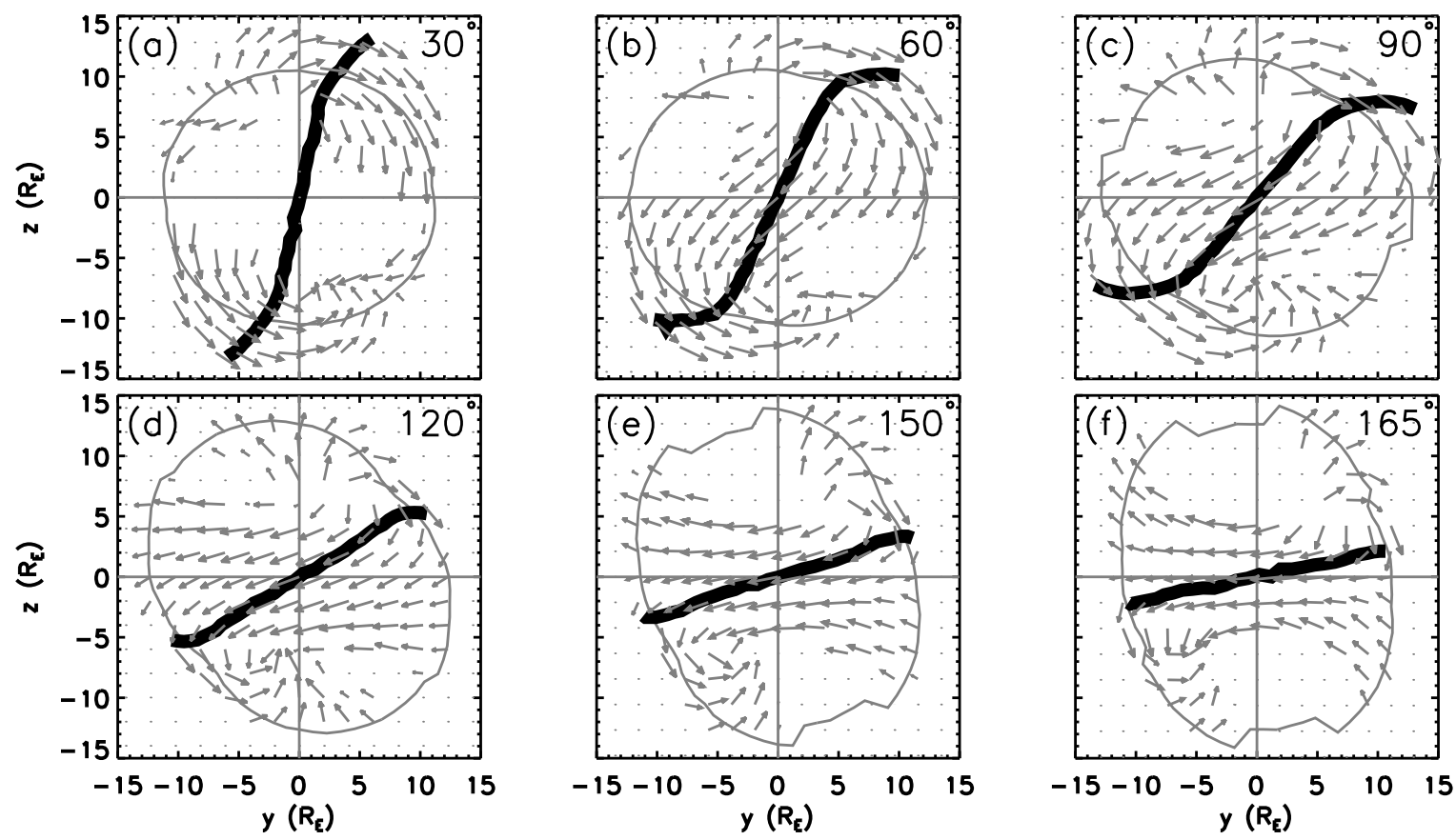

Figure 4.9: Results for the uniform $B_{M}$ component model. Panels (a) through (f) are from the same simulations as in Figures 4.4-4.8. The $y$ and $z$ components of $B_{M}$ are plotted as gray arrows and the magnetic separator is displayed in black.

The uniform $B_{M}$ component model would accurately predict the separator if the arrows point along the separator. While some of the arrows displayed in Fig. 4.9 appear to visually approximate the separator at the subsolar point $(0,0)$, overall there is not very strong agreement with this model. We quantify this result by calculating the orientation angle $\alpha$ of $\hat{\mathbf{M}}$ relative to the magnetospheric magnetic field (see Fig. 4.1): (a) N/A (reconnection is geometrically impossible), (b) $46.9^{\circ}$, (c) $53.1^{\circ}$, (d) $64.1^{\circ}$, (e) $76.8^{\circ}$, and (f) $83.6^{\circ}$. These angles are inconsistent with the orientation angles $\alpha_{\text {Sep }}$ of the magnetic separators (a) $10.3^{\circ}$, (b) $21.7^{\circ}$, (c) $35.8^{\circ}$, (d) $55.3^{\circ}$, (e) $71.2^{\circ}$, and (f) $77.5^{\circ}$. The discrepancies between the magnetic field's $M$ component and the orientation of the magnetic separators provide further evidence that this description does not accurately predict the orientation of magnetic reconnection, consistent with previous observations (Teh and Sonnerup, 2008) and simulations (Schreier et al., 2010). 


\subsubsection{IMF Strength Dependence}

Equations (4.4) and (4.6) have strong dependencies on the magnetospheric and magnetosheath magnetic field strengths. The magnetospheric magnetic field strength is usually much larger than that of the magnetosheath, and does not vary much for the chosen simulation parameters. This implies that the reconnection efficiency models depend strongly on the magnetosheath magnetic field strength $B_{\mathrm{SH}}$. Meanwhile, the maximum magnetic shear model is expected to be independent of $B_{\mathrm{SH}}$. We therefore vary $B_{\mathrm{SH}}$ to see if it helps to distinguish among these models.

We lower the IMF strength from $20 \mathrm{nT}$ to 5 and $2 \mathrm{nT}$ in separate simulations and use $\theta_{\mathrm{IMF}}=120^{\circ}$ with all other simulation parameters unchanged. Figure 4.10 displays the results from these simulations. The top row displays the magnetic shear angle in degrees, the middle row has the asymmetric outflow speed in $\mathrm{km} / \mathrm{s}$, and the bottom row has the asymmetric Sweet-Parker reconnection rate in $\mathrm{mV} / \mathrm{m}$. Figures $4.10(\mathrm{a}),(\mathrm{d})$, and (g) are for the $B_{\mathrm{IMF}}=20 \mathrm{nT}$ simulation, (b), (e), and (h) have $B_{\mathrm{IMF}}=5 \mathrm{nT}$, and (c), (f), and (i) use $B_{\mathrm{IMF}}=2 \mathrm{nT}$. As before, each model's prediction is plotted as the gray squares, the magnetic separators are displayed in white.

We note in passing that the magnetopause is elongated along the IMF direction in the $20 \mathrm{nT}$ plots of Fig. 4.10. This effect is consistent with observations (Lavraud et al., 2013) and simulations (Lavraud and Borovsky, 2008) for low solar wind Alfvén Mach number.

The subsolar region in each simulation becomes increasingly asymmetric as the IMF strength is lowered; the measured magnetic field ratios are $b=B_{\mathrm{MS}} / B_{\mathrm{SH}}=1.4,4.1$, and 11 for $B_{\mathrm{IMF}}=20,5$, and $2 \mathrm{nT}$, respectively. As seen in Fig. 4.10, each model's prediction becomes noisier for smaller $B_{\mathrm{SH}}$ due to the limitation of determining the magnetopause via the current maximum, as described in Section 4.2.1. 

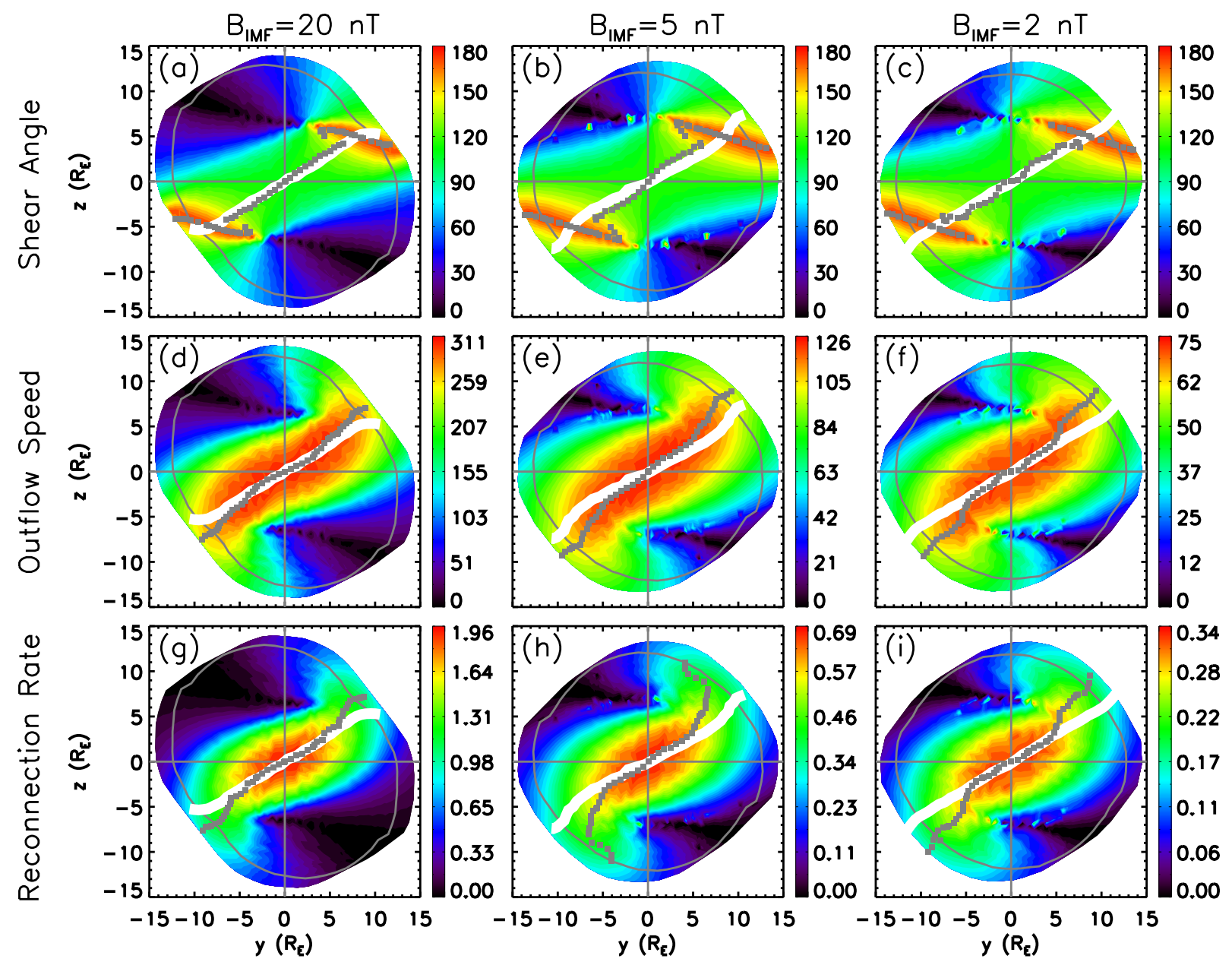

Figure 4.10: Results for testing model dependence on IMF strength for: (top row) the magnetic shear angle, (middle row) asymmetric outflow speed, and (bottom row) the asymmetric SweetParker reconnection rate. Plots (a), (d) and (g) are for $B_{\mathrm{IMF}}=20 \mathrm{nT},(\mathrm{b}),(\mathrm{e}),(\mathrm{h})$ are for $B_{\mathrm{IMF}}=5 \mathrm{nT}$, and (c), (f), (i) for $B_{\mathrm{IMF}}=2 \mathrm{nT}$. See the caption of Figure 4.4 for definitions.

Figures 4.10(a)-(c) show the maximum magnetic shear model remains relatively independent of the IMF strength as expected; this model's predictions have very similar structures as seen previously for arbitrary $\theta_{\mathrm{IMF}}$, making similar angles with the magnetospheric magnetic field of $49.6^{\circ}, 50.2^{\circ}$, and $53.2^{\circ}$ in simulations with $B_{\mathrm{IMF}}=20,5$, and $2 \mathrm{nT}$, respectively. This model maps large portions of the magnetic separator across the dayside magnetopause, consistent with previous results.

It is difficult to see differences between the asymmetric outflow speed and asymmetric 
Sweet-Parker reconnection rate models in Fig. 4.10. The predictions of both models closely map the magnetic separator in these simulations. For a more quantitative comparison between these models, we calculate each model's predicted orientation angle and compare these values to the separator's orientation at the subsolar point. The separator's orientation angle $\alpha_{\text {Sep }}$ measured from the magnetospheric magnetic field is $55.3^{\circ}, 61.5^{\circ}$, and $56.2^{\circ}$ in the $B_{\mathrm{IMF}}=20,5$, and $2 \mathrm{nT}$ simulations, respectively. The orientation of the ridges passing through the subsolar region for the asymmetric outflow speed make an angle $\alpha_{V}$ and, likewise, the asymmetric Sweet-Parker reconnection rate $\alpha_{E}$ are again measured from the magnetospheric magnetic field, with values $\alpha_{V}=47.0^{\circ}$ and $\alpha_{E}=51.0^{\circ}$ in the $B_{\mathrm{IMF}}=20 \mathrm{nT}$ simulation, $\alpha_{V}=45.7^{\circ}$ and $\alpha_{E}=49.7^{\circ}$ for $B_{\mathrm{IMF}}=5 \mathrm{nT}$, and finally, $\alpha_{V}=47.4^{\circ}$ and $\alpha_{E}=47.4^{\circ}$ with $B_{\mathrm{IMF}}=2 \mathrm{nT}$.

The orientation angle $\alpha_{E}$ decreases as IMF strength is lowered, while $\alpha_{V}$ displays a minimum when $B_{\mathrm{IMF}}=5 \mathrm{nT}$. However, $\alpha_{\text {Sep }}$ is maximum when $B_{\mathrm{IMF}}=5 \mathrm{nT}$. All of the models tested have predictions that are reasonably close, but none show perfect agreement with the orientation at the subsolar point. This implies that these models will need further modification to accurately predict the orientation of reconnection at Earth's subsolar magnetopause.

\subsubsection{Dipole Tilt Dependence}

Including a dipole tilt can alter the location of magnetic reconnection. For example, Trattner et al. (2007) showed that the prediction of the maximum magnetic shear model moves southward under positive dipole tilt (as measured from the $+z$ axis in the $x$ - $z$ GSM plane, i.e. the northern magnetic pole pointing Sunward), and likewise moves northward for negative dipole tilt when paired with southward IMF orientations. Reconnection in simulations 

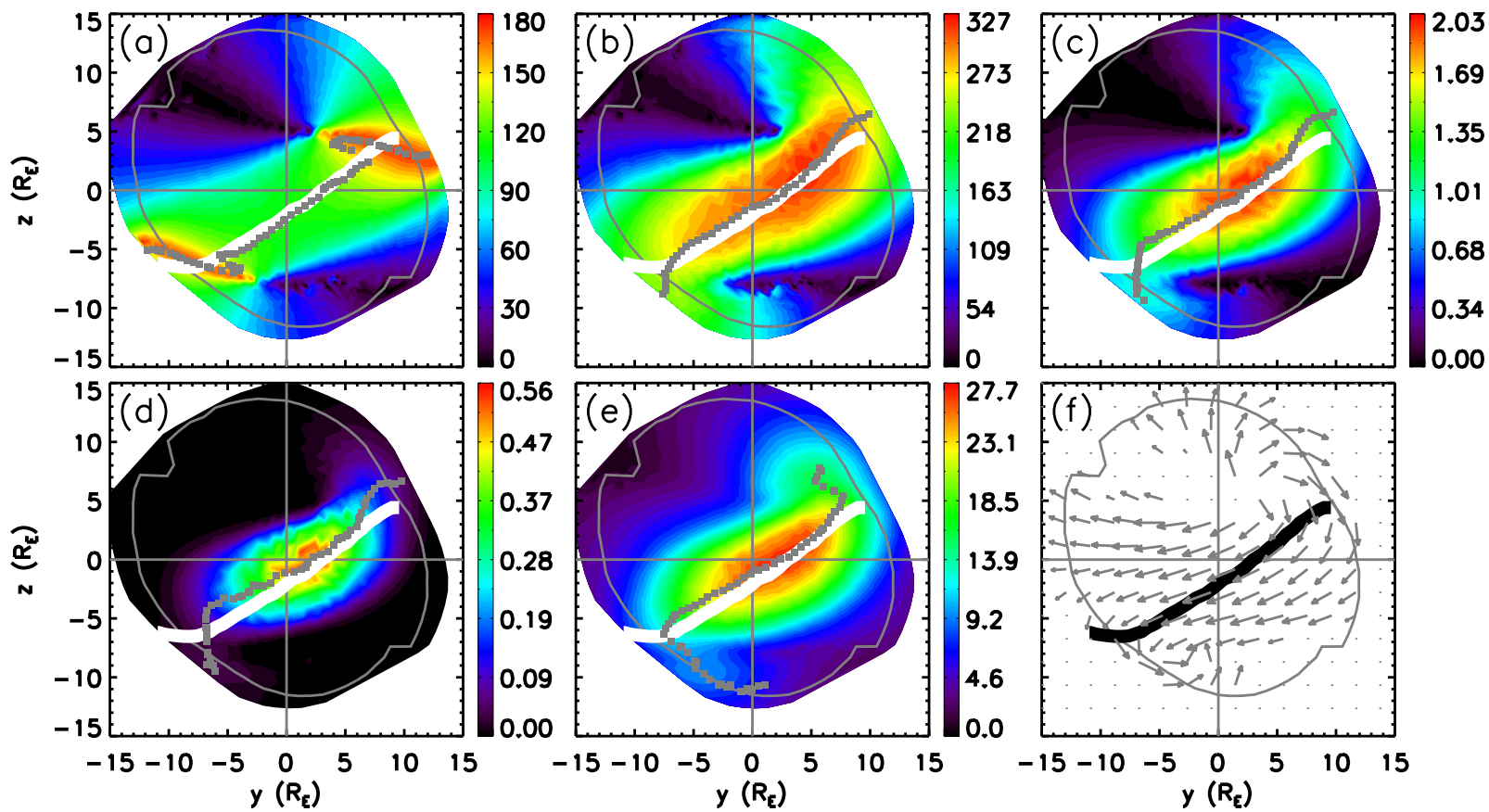

Figure 4.11: Results from a simulation with a dipole tilt of $+15^{\circ}$ and a southward IMF orientation with clock angle $120^{\circ}$ : (a) the magnetic shear angle $\theta$, (b) the asymmetric outflow speed $c_{A, \text { out }}$, (c) the asymmetric Sweet-Parker reconnection rate $E_{S P}$, (d) the angle of bisection, (e) the current density magnitude $|\mathbf{J}|$, and (e) the $y$ and $z$ components of the uniform $B_{M}$ component. See the caption of Figure 4.4 for definitions.

has also been measured to change location in simulations with a dipole tilt (Park et al., 2006; Palmroth et al., 2012; Cnossen et al., 2012; Hoilijoki et al., 2014).

Employing a more realistic dipole model of Earth may assist in distinguishing among the models. Note in Figs. 4.4-4.8 the model predictions intersect the magnetic separators at the subsolar point and near the anti-parallel region for all IMF clock angles. These intersections result from symmetry; including a dipole tilt will break this symmetry making it easier to distinguish which model predicts the magnetic separator.

We present the results of two additional simulations that employ a $+15^{\circ}$ dipole tilt. The same solar wind parameters described in Chapter 2 are used in separate simulations with IMF clock angles of $120^{\circ}$ (Fig. 4.11) and $30^{\circ}$ (Fig. 4.12). Figures 4.11 and 4.12 display each model's prediction as the grey squares, where the maximized quantity is: (a) the magnetic 

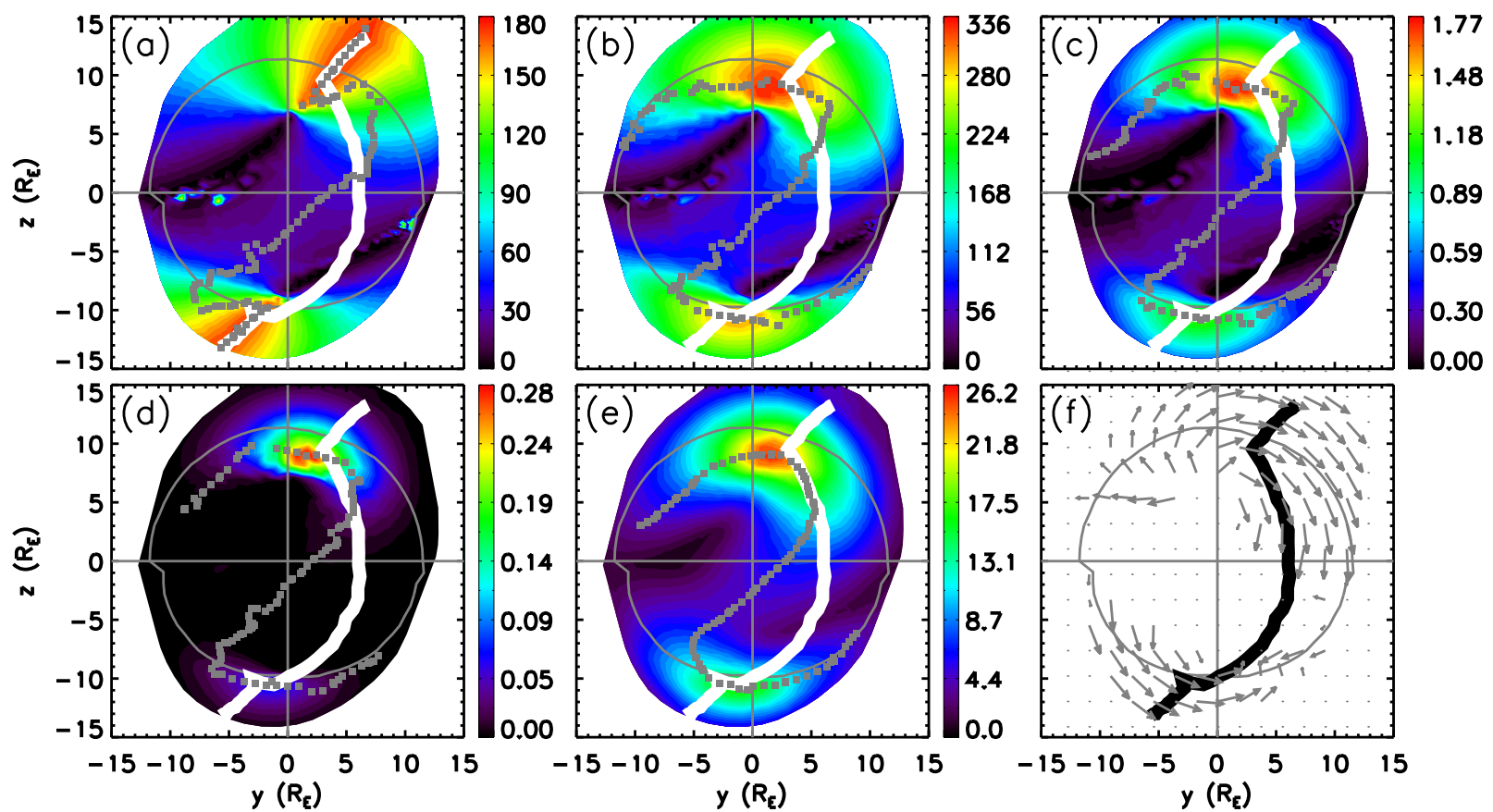

Figure 4.12: Results from a simulation with dipole tilt of $+15^{\circ}$ and a northward IMF orientation with clock angle $30^{\circ}$. See the caption of Figure 4.11 for definitions.

shear angle $\theta$ in degrees, (b) the asymmetric outflow speed $c_{A, \text { out }}$ in $\mathrm{km} / \mathrm{s},(\mathrm{c}$ ) the asymmetric Sweet-Parker reconnection rate $E_{S P}$ in $\mathrm{mV} / \mathrm{m},(\mathrm{d})$ the quantity $B_{\mathrm{MS}, L}^{2} B_{\mathrm{SH}, L}^{2}$ from Eq. (4.7) in $10^{8} \mathrm{nT}^{4}$, and (e) the current density magnitude $|\mathbf{J}|$ in $\mathrm{nA} / \mathrm{m}^{2}$, and (f) the projection of the uniform $B_{M}$ component in the $x=0$ plane. The magnetic separators are displayed in white for panels (a)-(e) and black in panel (f) as before.

In Fig. 4.11, each model appears to map the magnetic separator reasonably well with a southward IMF orientation and positive dipole tilt. The maximum magnetic shear model's prediction follows the separator more closely than any other model when the dipole tilt is present. The other models map the separator more closely in the northern, dusk quadrant in Figs. 4.11(b)-(e) than any other quadrant. [We use dawn $(-y)$, dusk $(+y)$, southern $(-z)$, northern $(+z)$ to indicate position in the $y$ - $z$ GSM plane.] Figure 4.11(f) shows that the uniform $B_{M}$ component model again fails to map the magnetic separator in any quadrant. 
The results for IMF clock angle $30^{\circ}$ and positive dipole tilt in Fig. 4.12 are significantly different. The separator moves approximately $45^{\circ}$ in longitude duskward of the $+x_{\mathrm{GSM}}$ axis. The model predictions intersect the magnetic separator in the northern-dusk and southerndawn quadrants, with the maximum current density mapping the largest portion of the separator in the northern-dusk quadrant. The maximum magnetic shear model is again the only model that faithfully reproduces the nightward portion of the magnetic separator. Most importantly, no model maps any part of the separator in the southern, dusk quadrant.

\subsubsection{Effect of a Flow Shear}

Existing models have thus far ignored the impact of solar wind flow around the magnetopause. The tailward flow of magnetosheath plasma around the magnetopause can have a component parallel to the reconnecting component of the magnetic field, which is known to impact the efficiency of reconnection (Chen and Morrison, 1990; La Belle-Hamer et al., 1994, 1995; Cassak and Otto, 2011). This effect potentially could alter models based on reconnection efficiency.

The incorporation of a plasma flow shear into equations (4.4) and (4.6) is ongoing work. We hypothesize that the predictions for the outflow speed (Cassak, 2011) and reconnection rate (Cassak and Otto, 2011) for symmetric reconnection with a flow shear can be gener-

alized for asymmetric reconnection by replacing the symmetric outflow speed $c_{A}$ with the asymmetric outflow speed $c_{A, \text { out }}$ from Eq. (4.4), giving

$$
c_{\text {Shearflow }}=c_{A, \text { out }} \sqrt{1-\frac{v_{s}^{2}}{c_{A, \text { out }}^{2}}},
$$


and

$$
E_{\text {Shearflow }}=E_{S P}\left(1-\frac{v_{s}^{2}}{c_{A, \text { out }}^{2}}\right),
$$

where $E_{S P}$ is given by Eq. (4.6). The flow shear speed $v_{s}$ is given by

$$
v_{s}=\frac{v_{\mathrm{SH}, L}-v_{\mathrm{MS}, L}}{2}
$$

and $v_{\mathrm{SH}, L}$ and $v_{\mathrm{MS}, L}$ are the magnetosheath and magnetopause flow in the $L$ direction, respectively.

We test to see if these expressions alter the predictions of the reconnection efficiency arguments. As before, $\alpha$ is discretized to calculate the asymmetric arguments $c_{A, \text { out }}$ and $E_{S P}$ while the $L$ direction is rotated around the magnetopause normal for each $\alpha$. The velocities on each side of the magnetopause are measured $3 \delta$ upstream along $\hat{\mathbf{n}}$ and $v_{\mathrm{SH}, L}$ and $v_{\mathrm{MS}, L}$ are calculated by taking the $L$-component for each $\alpha$. We calculate the modified reconnection outflow speed and electric field using equations (4.10) and (4.11) as a function of $\alpha$. (For $v_{s}>c_{A, \text { out }}, c_{\text {Shearflow }}$ and $E_{\text {Shearflow }}$ are set to zero as reconnection should not be possible.) Modified model predictions are determined with image processing techniques as described in Section 4.2.2.

Figure 4.13 displays results for two different clock angles: the top row are from the simulation with $\theta_{\mathrm{IMF}}=30^{\circ}$ and the bottom row has $\theta_{\mathrm{IMF}}=120^{\circ}$. Panels $(\mathrm{a})$ and $(\mathrm{e})$ are results without corrections for flow shear and are replicated from Fig. 4.5. Likewise, panels (c) and (g) are from Fig. 4.6 for the reconnection rate. Panels (b) and (f) give results that incorporate a flow shear in the calculation of the outflow speed and (d) and (h) its incorporation in the reconnection rate. The model predictions more closely map the separator when the IMF has a northward orientation after accounting for the flow shear, 

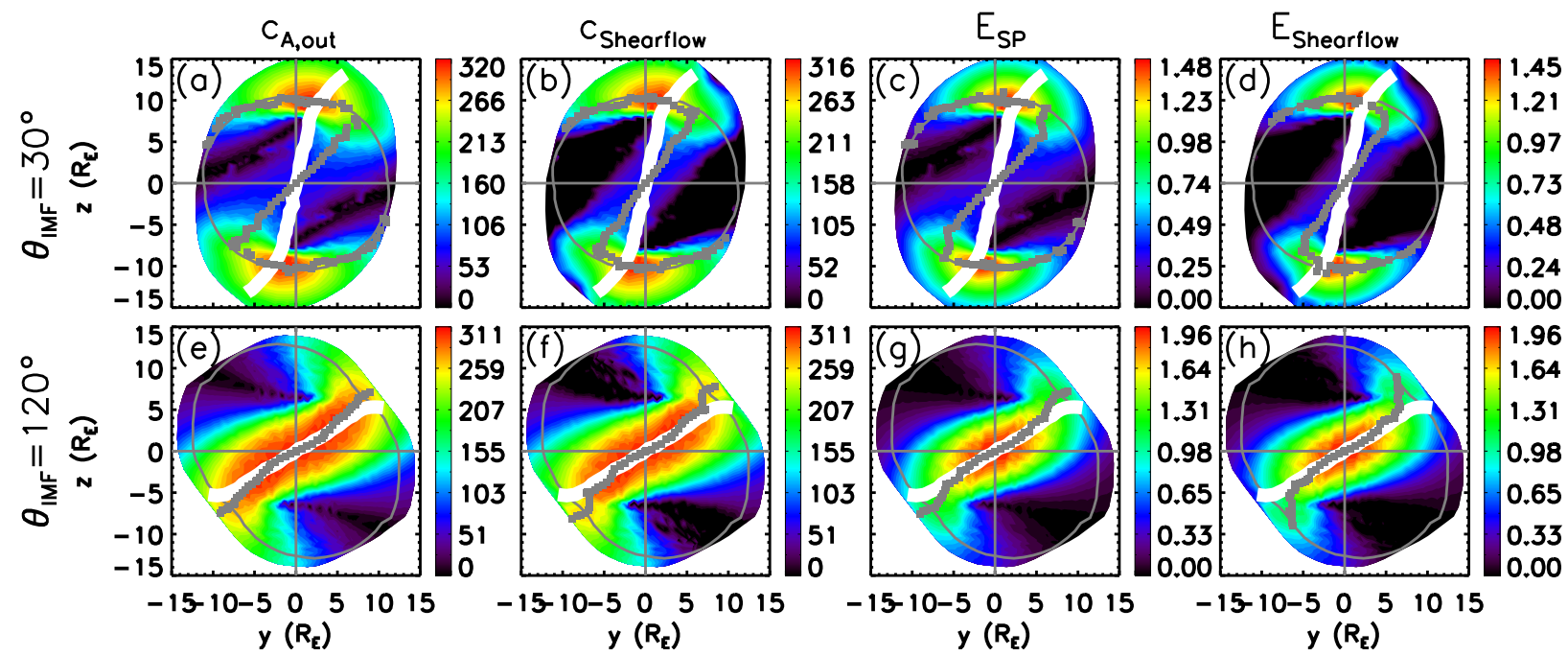

Figure 4.13: Results for testing the effects of flow shear on model predictions with (top row) $\theta_{\mathrm{IMF}}=$ $30^{\circ}$ and (bottom row) $120^{\circ}$. Plots (a) and (e) are for the unmodified asymmetric outflow speed $c_{A, \text { out }}$, (b) and (f) are for the modified outflow speed $c_{\text {Shearflow }},(\mathrm{c})$ and $(\mathrm{g})$ are for the unmodified asymmetric Sweet-Parker reconnection rate $E_{S P}$, and (d) and (h) are for the

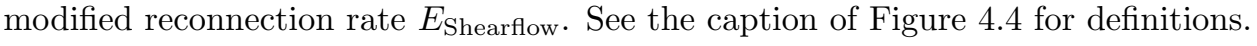

and remain largely unchanged with a southward IMF orientation. Quantifying this effect in the northward IMF simulation, the prediction for $c_{A, \text { out }}$ makes an angle $\alpha$ of $44.7^{\circ}$ with the magnetospheric magnetic field, and changes to $32.7^{\circ}$ after accounting for the flow shear; the separator makes an angle of $\alpha_{S e p}=10.3^{\circ}$. Likewise, the prediction of $E_{S P}$ makes an angle of $43.2^{\circ}$ and is $29.7^{\circ}$ after including a flow shear. Changes to the orientation angles for each model's prediction in the southward IMF simulation are much smaller changing from $47.0^{\circ}$ to $46.4^{\circ}$ for the outflow speed, and change from $51.0^{\circ}$ to $52.1^{\circ}$ for the reconnection electric field; the separator has $\alpha_{S e p}=55.3^{\circ}$ for this simulation. We note that these changes are not significant in light of the uncertainties inherent to the ridge detection algorithm at the subsolar magnetopause as discussed at the beginning of Section 4.3.1. A further test of this model was performed for the $30^{\circ}$ clock angle with positive dipole tilt simulation. The results (not shown) revealed improvement of the model predictions in some areas, and worse agreement in others, but still differed significantly from the separator. 
The relative importance of a flow shear is understood as a result of general flow patterns around the magnetopause. For southward IMF, reconnection occurs near the ecliptic plane where the magnetosheath flow and magnetospheric convection are tangential to this plane. Thus, any flow shear parallel to the magnetic field is small and reconnection should not be strongly affected. However, the magnetosheath flow at high latitudes is quite large. When the IMF is northward, there is appreciable flow parallel to the reconnecting magnetic fields and may lead to the suppression of reconnection. The present results suggest that including flow shear improves the agreement between separators and model predictions for the reconnection efficiency arguments.

We note that the analysis here assumes that any $v_{M}$ flow tangential to the $L$-components of the magnetic fields has no effect on the reconnection. This is unlikely to be the case, but including this is beyond the scope of the present study. We additionally note that recent results from a study with flow shear parallel to the reconnecting magnetic fields in asymmetric reconnection systems suggest that the model described here is incorrect (Doss et al., in prep.); the key results of this new work are presented in Chapter 1.4.3.

\subsection{Summary}

The present study calculates the predictions of several magnetic reconnection location models at the dayside magnetopause in global resistive MHD simulations. We note that even though the real magnetosphere is collisionless, the results of the present study should be independent of our choice to include an explicit resistivity as most of the reconnection location models discussed here are independent of reconnection's dissipation mechanism. We find that the maximum magnetic shear model (Trattner et al., 2007) best predicts the magnetic separator 
for northward IMF orientations, which occurs near the polar cusps. For southward IMF, most of the models tested give results fairly close to the location of dayside reconnection, but none are perfect. We conclude that the existing models may be good enough for a ballpark estimate, but it remains unclear what physically controls the location of magnetic reconnection at the dayside magnetopause. 


\section{Chapter 5}

\section{Local Analysis of Reconnection at the Dayside Magnetopause}

There is ongoing debate whether dayside reconnection is entirely controlled by the conditions imposed by the magnetosphere's external driver, the solar wind, or whether the plasma parameters local to the magnetopause have an effect. While observations and simulations have shown the relative importance of local physics determining the local reconnection dynamics (Borovsky et al., 2008; Walsh et al., 2014), it is not presently understood how the local reconnection dynamics affect the larger magnetospheric dynamics.

From our point of view, in order to systematically study this issue, one must first carefully analyze the reconnection physics local to the magnetic separator. This approach has been used in the solar context before (Parnell et al., 2010), in which the authors analyzed reconnection local to self-consistently generated separators in a resistive MHD code. The authors demonstrated that X-point magnetic field geometries typical of reconnection and reconnection outflows appear in planes perpendicular to the magnetic separator. 
Having located the magnetic separators in our global magnetospheric resistive MHD simulations, we therefore employ a similar methodology in order to quantify reconnection local to the dayside portions of the magnetic separators. We go beyond previous work by systematically measuring local parameters in each separator plane and comparing the results to local reconnection theory predictions of the reconnection rate. As reconnection at the dayside magnetopause is inherently asymmetric, it is natural to test the predictions of Section 1.4.2 to assess this theory's capability of predicting the separator's reconnection rate. Previous studies have presented scaling studies of anti-parallel asymmetric reconnection in 2D slab geometries in resistive MHD (Cassak and Shay, 2007), two-fluid Hall MHD (Cassak and Shay, 2008, 2009), and in particle-in-cell (Malakit et al., 2010) simulations; the asymmetric reconnection predictions were shown to perform well in 2D. However, the theory is manifestly $2 \mathrm{D}$, having been derived in $2 \mathrm{D}$ and tested in $2 \mathrm{D}$ simulations. Therefore, it is not fully understood if it can be applicable to the fully 3D magnetosphere (J. Dorelli, GEM Summer Workshop, 2014). While attempts have been made to determine the applicability to the fully 3D magnetopause for due southward IMF (Borovsky et al., 2008; Ouellette et al., 2014), we are not aware of any studies applying these relations to IMF oriented obliquely with the geomagnetic dipole.

For our base simulation parameters with southward IMF orientations $\theta_{\mathrm{IMF}} \geq 90^{\circ}$, we find that local Sweet-Parker asymmetric reconnection theory does surprisingly well at predicting the scaling of the rate of reconnection at the separator; this result even holds for the separator arising in a simulation with a dipole tilt. Agreement between the two breaks down once the IMF strength is lowered from our base simulation parameters. We argue that the breakdown in agreement is numerical in nature and not a failure of the local reconnection theory.

The layout of this chapter is as follows. Section 5.1 describes the systematic approach 
used to measure local plasma parameters of the magnetosphere and magnetosheath which are used to calculate the local reconnection rate scaling relations. We present the results of our simulations in Section 5.2. A brief summary of our results are presented in Section 5.3.

\subsection{Methodology}

\subsubsection{The Determination of Planes Normal to the Separator}

The separator tracing algorithm presented in Chapter 3 results in a number of locations lying along the separator. We develop a procedure to construct planes normal to the separator by defining an orthonormal basis at every point along the separator from the known points on the separator and their positions relative to Earth. As a motivation for the procedure, consider the separator in our $\theta_{\mathrm{IMF}}=90^{\circ}$ base simulation, replotted in Fig. 5.1(a). The plane centered normal to the separator at the subsolar point $\mathbf{r}_{S e p}=(8.4,0.0,0.0)$ is sketched as the dashed line. We define a coordinate system $\left(x^{\prime}, y^{\prime}, z^{\prime}\right)$ for this plane. The out-of-plane unit vector $\hat{\mathbf{z}}^{\prime}$ points along the separator, i.e., along the magnetic field with $\hat{\mathbf{z}}^{\prime}=0.62 \hat{\mathbf{y}}+0.78 \hat{\mathbf{z}}$, with unprimed vectors given in GSM. We define the $y^{\prime}$ direction as the inflow direction and $x^{\prime}$ as the outflow direction. For the case study, the inflow direction at the subsolar point is aligned with the solar wind flow, so $\hat{\mathbf{y}}^{\prime}=\hat{\mathbf{x}}$. Finally, the in-plane unit vector completing the orthonormal triplet is defined by $\hat{\mathbf{x}}^{\prime}=\hat{\mathbf{y}}^{\prime} \times \hat{\mathbf{z}}^{\prime}$.

With this coordinate system in mind, we now describe the method by which we determine this coordinate system at an arbitrary point on the separator. For the $k$-th location along the separator, the out-of-plane unit vector $\hat{\mathbf{z}}_{k}^{\prime}$ is tangent to the separator. Using a second 

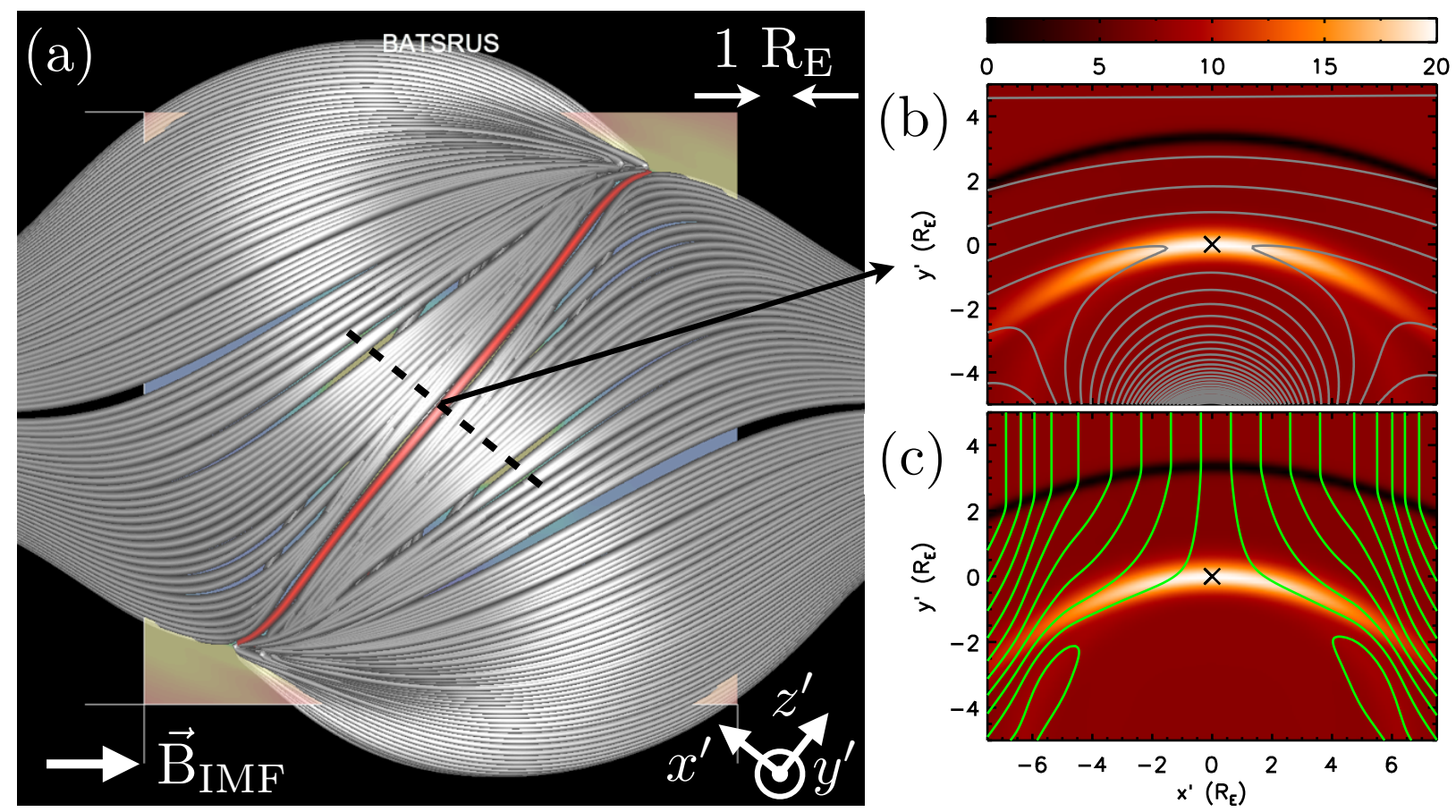

Figure 5.1: Results of determining the plane normal to the separator at the subsolar magnetopause in a simulation with $\theta_{\mathrm{IMF}}=90^{\circ}$. Panel (a) is reproduced from Fig. 1.13, and depicts the orientation of the separator plane centered at $\mathbf{r}_{S e p}=(8.4,0.0,0.0) \mathrm{R}_{\mathrm{E}}$ in GSM coordinates (dashed black line). Panels (b) and (c) display the out-of-plane current density $J_{z^{\prime}}$ as the color background in $\mathrm{nA} / \mathrm{m}^{2}$ and displays contours of (b) the flux function $\Psi$ in gray and (c) contours of the stream function $\Phi$ in green. The separator is located at $(0,0)$ in the $x^{\prime}-y^{\prime}$ plane and marked with an $\mathrm{X}$.

order finite difference, this gives

$$
\hat{\mathbf{z}}_{k}^{\prime}=\frac{\mathbf{r}_{k-1}-\mathbf{r}_{k+1}}{\left|\mathbf{r}_{k-1}-\mathbf{r}_{k+1}\right|}
$$

where $\mathbf{r}_{k-1}$ and $\mathbf{r}_{k+1}$ are the previous and subsequent separator locations, respectively. We note that one could think of defining $\hat{\mathbf{z}}_{k}^{\prime}$ by the magnetic field direction $\hat{\mathbf{b}}$ at the separator location, but this definition fails when the magnetic field parallel to the separator is small, such as for due southward $\operatorname{IMF}\left(\theta_{\mathrm{IMF}}=180^{\circ}\right)$. The formulation of Eq. (5.1) guarantees a meaningful $\hat{\mathbf{z}}^{\prime}$ for any IMF orientation and magnetospheric dipole tilt.

The unit vector $\hat{\mathbf{y}}_{k}^{\prime}$ in the direction of the inflow is given by the normal to the magne- 
topause at $\mathbf{r}_{k}$. This is calculated by finding the projection of $\mathbf{r}_{k}$ normal to $\hat{\mathbf{z}}_{k}^{\prime}$. Mathematically, this is represented as

$$
\hat{\mathbf{y}}_{k}^{\prime} \propto \mathbf{r}_{k}-\left(\mathbf{r}_{k} \cdot \hat{\mathbf{z}}_{k}^{\prime}\right) \hat{\mathbf{z}}_{k}^{\prime}
$$

Finally, $\hat{\mathbf{x}}_{k}^{\prime}$ completes the right-handed triplet by taking the cross product

$$
\hat{\mathbf{x}}_{k}^{\prime}=\hat{\mathbf{y}}_{k}^{\prime} \times \hat{\mathbf{z}}_{k}^{\prime}
$$

With this orthonormal basis, the $x^{\prime}-y^{\prime}$ plane is expected to be the reconnection plane. Coordinates of locations in this plane are translated back to GSM coordinates, and Kameleon is used to sample the plasma number density $n$, thermal pressure $p$, magnetic field $\mathbf{B}$, plasma flow $\mathbf{u}$, and current density $\mathbf{J}$ in this plane. Each plane spans $-7.5 \leq x^{\prime} \leq 7.5$ and $-5 \leq$ $y^{\prime} \leq 5 \mathrm{R}_{\mathrm{E}}$ and each direction is sampled in $\Delta x^{\prime}=\Delta y^{\prime}=0.0625 \mathrm{R}_{\mathrm{E}}$ increments; the separator is centered at $(0,0)$ in each $x^{\prime}-y^{\prime}$ plane. Finally, the magnetic field $\mathbf{B}$, plasma flow $\mathbf{u}$, and current density $\mathbf{J}$ vectors are transformed from GSM coordinates to primed coordinates of the separator plane, e.g., $B_{x^{\prime}}=\mathbf{B} \cdot \hat{\mathbf{x}}^{\prime}$.

We return to the separator in our base simulation with $\theta_{\mathrm{IMF}}=90^{\circ}$ in Fig. 5.1 where panel (a) sketches the plane through the subsolar point with a dashed black line. Panels (b) and (c) display the out-of-plane current density component $J_{z^{\prime}}$ as the color background in $\mathrm{nA} / \mathrm{m}^{2}$. The separator's location in the $x^{\prime}-y^{\prime}$ plane is marked with an $\mathrm{X}$ at $(0,0)$ in panels (b) and (c).

In order to gain insight into what reconnection might look like in this plane, we employ a method used in 2D geometries to determine the structure of the magnetic field that is not formally generalizable to 3D. (No conclusions are drawn from this; it is merely presented for perspective.) Consider a system that in $2 \mathrm{D}$ is independent of $z^{\prime}$. We define the flux function 
$\Psi\left(x^{\prime}, y^{\prime}\right)$ as

$$
\mathbf{B}=-\hat{\mathbf{z}}^{\prime} \times \nabla^{\prime} \Psi
$$

where the magnetic field $\mathbf{B}$ and derivatives $\nabla^{\prime}$ are only considered in the $x^{\prime}-y^{\prime}$ plane. We note that the flux function $\Psi$ is the $z^{\prime}$ component of the vector potential A. Since $\mathbf{B} \cdot \nabla^{\prime} \Psi=0$, lines of constant $\Psi$ represent magnetic field lines. By taking the vector product between $\hat{\mathbf{z}}^{\prime}$ and Eq. (5.4), one obtains $\nabla^{\prime} \Psi=\hat{\mathbf{z}}^{\prime} \times \mathbf{B}$, where $\hat{\mathbf{z}}^{\prime} \cdot \nabla^{\prime} \Psi=0$ since we are in 2D. Finally, as with determining an electrostatic potential from an electric field, $\Psi$ can be determined from the integral

$$
\Psi=\int d \vec{\ell} \cdot\left(\hat{\mathbf{z}}^{\prime} \times \mathbf{B}\right)=\int d \vec{\ell} \cdot\left(B_{y^{\prime}} \hat{\mathbf{x}}^{\prime}-B_{x^{\prime}} \hat{\mathbf{y}}^{\prime}\right)
$$

where $d \vec{\ell}$ is the path taken in evaluating the integral. Just as in electrostatics, $\Psi$ is independent of the path of integration in 2D. We calculate $\Psi$ by integrating from the lower, left corner of the $x^{\prime}-y^{\prime}$ plane, first along $x^{\prime}$, and then along $y^{\prime}$. Contours of constant $\Psi$, representing magnetic field lines, are the gray lines depicted in Fig. 5.1(b).

One can similarly define a 2D stream function $\Phi$ to obtain the streamlines (field lines of the velocity vector) in the $x^{\prime}-y^{\prime}$ plane with the simple substitution of $\Phi$ for $\Psi$, and velocity components $u_{x^{\prime}}$ and $u_{y^{\prime}}$ for $B_{x^{\prime}}$ and $B_{y^{\prime}}$ in Eq. (5.4). Figure 5.1(c) displays contours of constant $\Phi$ in green which give the in-plane streamlines.

Figure 5.1 displays several features that are qualitatively consistent with the local picture of 2D Sweet-Parker collisional reconnection (Parker, 1957; Sweet, 1958) discussed in Chapter 1.4.1, albeit occurring in a dipolar magnetic field instead of straight magnetic field lines. First, the out-of-plane current layer is thin and elongated. The reconnecting magnetic field components are also oppositely directed with the IMF pointing along $-\hat{\mathbf{x}}^{\prime}$ and is carried along $-\hat{\mathbf{y}}^{\prime}$ in the magnetosheath; the dipolar magnetic field points along $+\hat{\mathbf{x}}^{\prime}$ and slowly 
convects towards the magnetopause along $+\hat{\mathbf{y}}^{\prime}$. These magnetic fields undergo reconnection at the separator with newly reconnected magnetic flux located downstream of the separator, and displaying a curved X-point reconnection geometry similar to those presented in Chapter 1. Lastly, the plasma convects horizontally outward from the separator along $y^{\prime}$ with speeds $|\mathbf{u}| \approx 205 \mathrm{~km} / \mathrm{s}$, higher than the vertically directed magnetosheath flow speed $|\mathbf{u}| \approx 150 \mathrm{~km} / \mathrm{s}$. This suggests reconnection has a role in accelerating the outflowing plasma. Thus the plane normal to the separator at the subsolar magnetopause qualitatively resembles the $2 \mathrm{D}$ pictures of reconnection.

We note that the technique for determining the in-plane magnetic field and streamlines works remarkably well for the subsolar magnetopause, and most likely results from the high degree of symmetry in this simulation since it does not include a dipole tilt and that the IMF has reflection symmetry about $y=-z$.

\subsubsection{Measuring Plasma Parameters in Reconnection Planes}

Having described the procedure to obtain planes normal to the separator and the plasma parameters in these planes, we wish to analyze the reconnection in each plane. To do so, we need the plasma parameters just upstream of the dissipation region since this plasma and the magnetic field it carries serves as the free energy for reconnection.

We start by sampling the out-of-plane current density $J_{z^{\prime}}$ along $\hat{\mathbf{y}}^{\prime}$ at $x^{\prime}=0$ to determine the location of maximum current density $J_{\max }$. Note that $J_{\max }$ may not be located at the separator, as it would be for symmetric reconnection; it can be offset during asymmetric reconnection (Cassak and Shay, 2007), as discussed in Section 4.2.1. We define the full-width, half-max (FWHM) of the out-of-plane current density profile as the dissipation region's thickness $2 \delta$. We define $y_{\mathrm{SH}}^{\prime}$ and $y_{\mathrm{MS}}^{\prime}$ as the locations corresponding to the magnetosheath and 
magnetospheric edges of the out-of-plane current profile, respectively. The number densities $n$ and reconnecting magnetic field components $B_{x^{\prime}}$ of the magnetosheath and magnetosphere are measured at $\left(0, y_{\mathrm{SH}}^{\prime}+\delta\right)$ and $\left(0, y_{\mathrm{MS}}^{\prime}-\delta\right)$, respectively, in the $x^{\prime}-y^{\prime}$ plane.

We return to the separator plane displayed in Fig. 5.1 in order to demonstrate the procedure for measuring the upstream plasma parameters; the results are displayed in Fig. 5.2. Panel (a) displays the out-of-plane current density $J_{z^{\prime}}$ as the color background with a dashed white line at $x^{\prime}=0$ displaying the line along which the plasma parameters are sampled. Panel (b) shows the out-of-plane current density along $x^{\prime}=0$, with vertical dotted lines at the locations $y_{\mathrm{MS}}^{\prime}$ and $y_{\mathrm{SH}}^{\prime}$ where the current density has the value $0.5 J_{\max }$ and is marked with a horizontal dotted line. The separator's location is marked with a solid vertical line at $y^{\prime}=0$. The left dashed vertical lines mark $y_{\mathrm{MS}}^{\prime}-\delta$ where the magnetospheric plasma parameters are measured and the magnetosheath's plasma parameters are measured at $y_{\mathrm{SH}}^{\prime}+\delta$ marked by the right vertical dashed line. Panel (c) displays the reconnecting magnetic field components $B_{x^{\prime}}$ in $\mathrm{nT}$ and panel (d) displays the plasma number density $n$ in $\mathrm{cm}^{-3}$, respectively. The locations where the upstream parameters are sampled are again displayed with vertical dashed lines; dashed horizontal lines in panels (c) and (d) display the sampled values. One can see that each determined parameter is representative of the asymptotic regions upstream of the dissipation region as desired. The upstream values for this plane on the magnetospheric side are $B_{x^{\prime}, \mathrm{MS}}=64 \mathrm{nT}$ and $n_{\mathrm{MS}}=11 \mathrm{~cm}^{-3}$ and for the magnetosheath plasma are $B_{x^{\prime}, \mathrm{SH}}=-61 \mathrm{nT}$, and $n_{\mathrm{SH}}=57 \mathrm{~cm}^{-3}$; the dissipation region has half-width $\delta=0.38 \mathrm{R}_{\mathrm{E}}$.

In order to check the validity of the asymmetric reconnection scaling relations, we must determine the half-length $L$ of the dissipation region. Care must be taken in determining the dissipation region length as it is curved due to the structure of Earth's magnetosphere. 

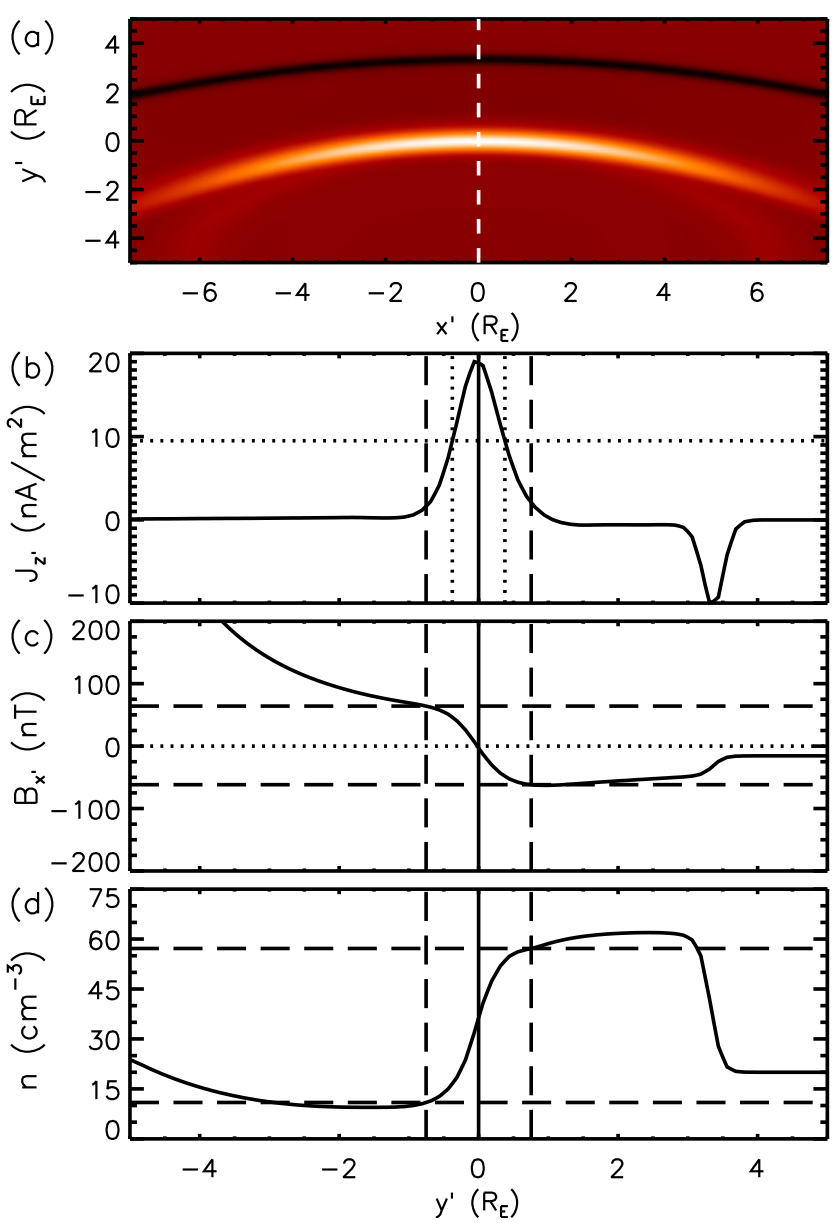

Figure 5.2: Determination of the upstream plasma parameters in a simulation with $\theta_{\mathrm{IMF}}=90^{\circ}$ for the plane normal to the separator located at the subsolar point. Panel (a) displays the out-of-plane current density $J_{z^{\prime}}$ and the white dashed line at $x^{\prime}=0$ displays the line along which plasma parameters are sampled. Panel (b) displays the out-of-plane current density along $x^{\prime}=0$ with the separator's location depicted by the solid line and locations where $J_{z^{\prime}}=0.5 J_{\max }$ displayed with vertical dotted lines. The value of $0.5 J_{\max }$ is marked by the horizontal dotted line. The vertical dashed lines in panels (b)-(d) indicate the locations where the magnetospheric and magnetosheath parameters are measured. Panel (c) displays the reconnecting magnetic field component $B_{x^{\prime}}$ in $\mathrm{nT}$, whereas panel (d) displays the plasma number density $n$ in $\mathrm{cm}^{-3}$; the horizontal lines in these plots mark the respective magnetospheric and magnetosheath values of these parameters. 
We therefore start by identifying the reconnection dissipation region by sampling the out-ofplane current density $J_{z^{\prime}}$ along cuts oriented at an angle $\theta$ from the $+x^{\prime}$ axis in the $x^{\prime}-y^{\prime}$ plane as displayed in Fig. 5.3; the cuts start at $(0,-5) \mathrm{R}_{\mathrm{E}}$ and the current density is sampled in $1 / 16 \mathrm{R}_{\mathrm{E}}$ increments, with $\theta$ discretized into $1^{\circ}$ increments from $\left[0^{\circ}, 180^{\circ}\right]$. The first current density maximum along each cut is retained. The right and left edges of the dissipation region are defined as $\theta_{\text {Right }}$ and $\theta_{\text {Left }}$ where the first current density maximum achieves a value of $J_{z^{\prime}}=0.5 J_{\max }$, where $J_{\max }$ is the maximum current density value along $x^{\prime}=0$, as described earlier in this section. $L$ is directly calculated from the arc length of the measured current density maxima locations as

$$
2 L=\int_{\theta_{\text {Right }}}^{\theta_{\text {Left }}} d S \simeq \sum_{k=\theta_{\text {Right }}}^{\theta_{\text {Left }}} \Delta S_{k}
$$

where $\Delta S_{k}$ is the distance between the $k$ th current density maximum at $\mathbf{S}_{k}$ and its neighbor at $\mathbf{S}_{k+1}$ given by

$$
\Delta S_{k}=\left|\mathbf{S}_{k+1}-\mathbf{S}_{k}\right|
$$

Figure 5.3 displays the results of this current density sampling method for the separator plane at the subsolar point for the $\theta_{\mathrm{IMF}}=90^{\circ}$ simulation. Panel (a) displays the out-ofplane current density $J_{y^{\prime}}$ as the color background and current density maxima are displayed with blue asterisks. Panel (b) plots the values of current density maxima in $\mathrm{nA} / \mathrm{m}^{2}$ as a function of the sampling angle $\theta$. Vertical dotted lines display the determined locations of $\theta_{\text {Left }}$ and $\theta_{\text {Right }}$, with the horizontal dotted line displaying $0.5 J_{\max }$. The dissipation region's half-length $L=5.84 \mathrm{R}_{\mathrm{E}}$ for this plane.

We have now measured all of the relevant parameters in order to make a meaningful comparison with the theoretical asymmetric reconnection scaling relations and the reconnection 

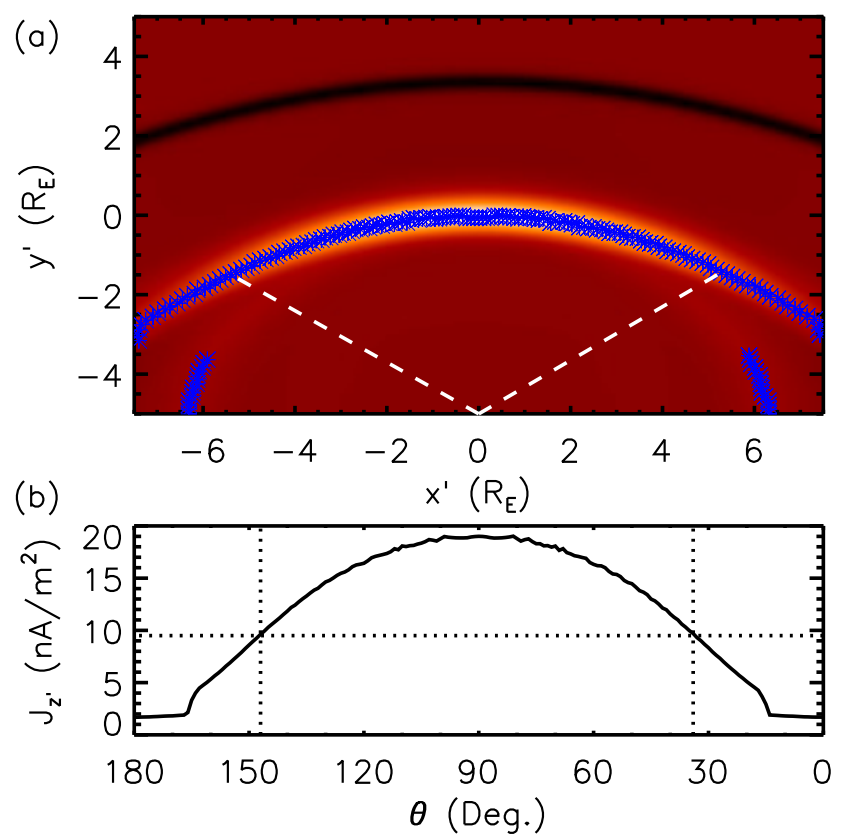

Figure 5.3: Results of determining the dissipation region half-length $L$ with maxima of the out-of-plane current density $J_{z^{\prime}}$ for the plane normal to the separator at the subsolar point in a simulation with $\theta_{\mathrm{IMF}}=90^{\circ}$. (a) The locations of current maxima are displayed as blue asterisks and (b) the current density values in $\mathrm{nA} / \mathrm{m}^{2}$ at each of these points as a function of sampling angle $\theta$ as measured from the $+x^{\prime}$ axis. The left and right edges $\theta_{\text {Left }}$ and $\theta_{\text {Right }}$ of the dissipation region are displayed as dotted vertical lines and are determined by where the current density falls to $0.5 J_{\max }$ as indicated by the horizontal dotted line.

rate measured in our global simulations. From the upstream reconnecting magnetic field components $B_{x^{\prime}}$ and densities $n$, the dissipation region's half-width $\delta$ and half-length $L$, we can calculate $E_{a s y m}$ and $E_{\eta, a s y m}$ from the asymmetric scaling relations given by Eqs. (1.22)(1.23), respectively. Both of these are compared with the reconnection rate at the separator $E_{z^{\prime}}=\eta J_{z^{\prime}}$. The procedure outlined here is repeated for all planes normal to the separator for all simulations in this study.

\subsection{Results}

Figure 5.4 displays the measured reconnection rate $E_{z^{\prime}}$ (black squares) along the separator in distinct simulations with (a) $\theta_{\mathrm{IMF}}=180^{\circ}$, (b) $150^{\circ}$, (c) $120^{\circ}$, and (d) $90^{\circ}$ employing 

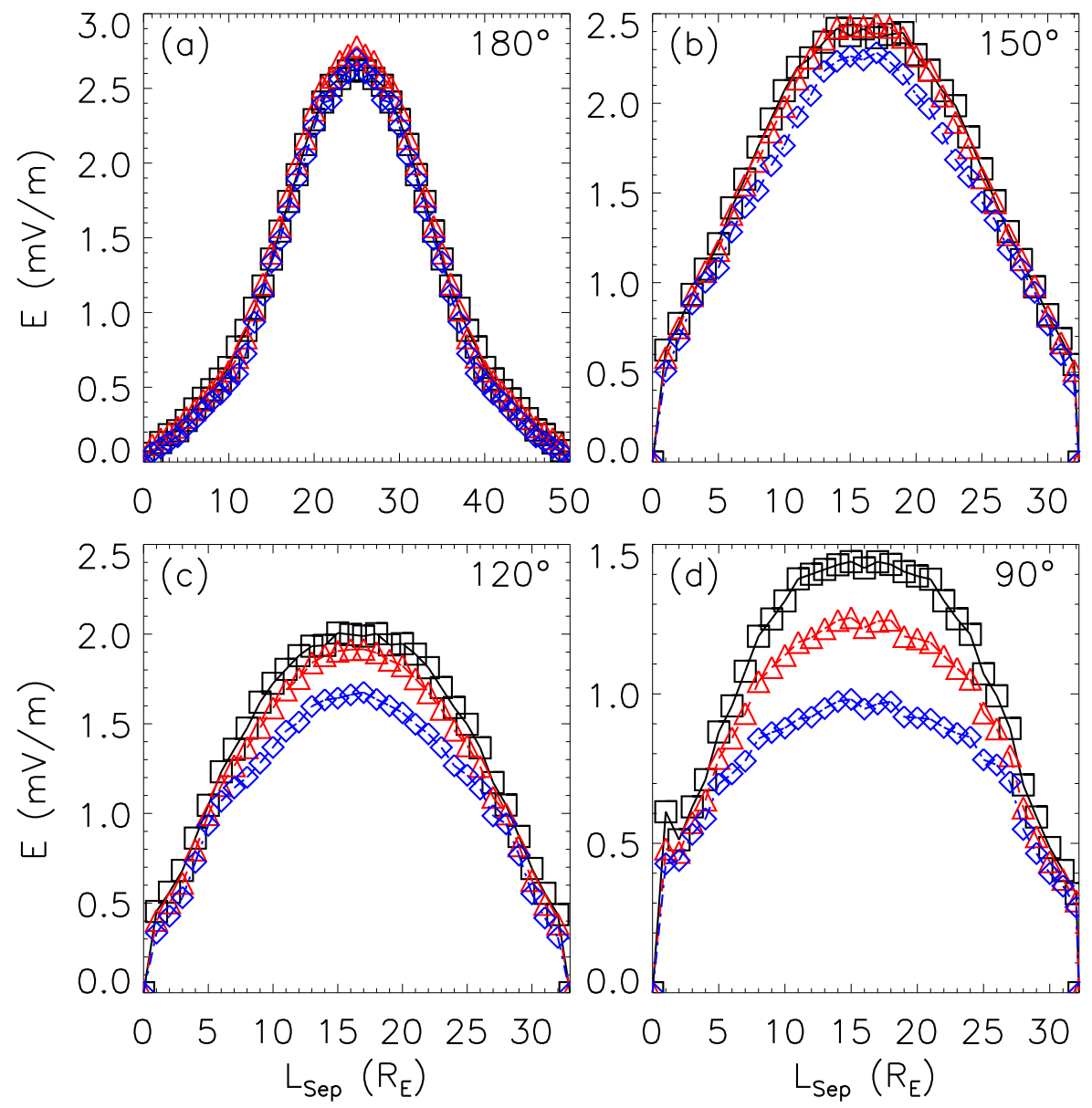

Figure 5.4: Results comparing the measured reconnection rate $E_{z^{\prime}}$ (black $\square$ ) at the separator with the theoretical $E_{\text {asym }}$ (blue $\diamond$ ) and the Sweet-Parker $E_{\eta, \text { asym }}($ red $\triangle$ ) asymmetric reconnection rates in distinct simulations with (a) $\theta_{\mathrm{IMF}}=180^{\circ}$, (b) $150^{\circ}$, (c) $120^{\circ}$, and (d) $90^{\circ}$. All simulations use our base simulation parameters. Electric fields are plotted as a function of $L_{S e p}$, the distance in $\mathrm{R}_{\mathrm{E}}$ along the separator as measured from the northern magnetic null.

our base simulation parameters. The measured reconnection rate in $\mathrm{mV} / \mathrm{m}$ is compared with the theoretical asymmetric reconnection rates $E_{\text {asym }}$ (blue diamonds) and $E_{\eta, a s y m}$ (red triangles) given by Eqs (1.22) and (1.23), respectively. The reconnection rates are plotted as a function of $L_{S e p}$, the distance along the separator as measured from the northern magnetic null measured in $R_{E}$. Note the vertical scale is different for different $\theta_{\mathrm{IMF}}$, consistent with reconnection being faster for larger $\theta_{\mathrm{IMF}}$.

The results for $\theta_{\mathrm{IMF}}=180^{\circ}$ in panel (a) are exceedingly good, as the theoretical and 
measured values are almost indistinguishable. This is consistent with the results of Borovsky et al. (2008) and Ouellette et al. (2014), who tested the theoretical predictions for due southward IMF. The other clock angle simulations in panels (b)-(d) reveal good agreement in the scaling sense, meaning that all parameters differ by some coefficient that is approximately constant along the entire separator. While the scaling is strong for all simulations with a southward component of the IMF, a comparison of absolute quantities shows that the quantitative agreement becomes progressively worse as the clock angle decreases.

As a way of quantifying the discrepancy between the measured reconnection rate and the predictions, the percent difference between the measured reconnection rate $E_{z^{\prime}}$ and the generalized asymmetric reconnection rate $E_{\text {asym }}$ is calculated as a function of the distance along the separator as

$$
\% \text { Error }=\frac{E_{a s y m}-E_{z^{\prime}}}{E_{z^{\prime}}} \times 100
$$

and for the asymmetric Sweet-Parker reconnection rate $E_{\eta, a s y m}$ as

$$
(\% \text { Error })_{\eta}=\frac{E_{\eta, a s y m}-E_{z^{\prime}}}{E_{z^{\prime}}} \times 100
$$

Using these equations, we calculate the average percent difference along the middle third of the separator, those locations between $10 \leq L_{S e p} \leq 20 \mathrm{R}_{\mathrm{E}}$ for $\theta_{\mathrm{IMF}}=90^{\circ}, 120^{\circ}$, and $150^{\circ}$, and $16 \leq L_{S e p} \leq 33 \mathrm{R}_{\mathrm{E}}$ for $\theta_{\mathrm{IMF}}=180^{\circ}$ for which $E$ is significantly different from zero and the percent error is relatively constant with distance along the separator. The average percent errors are $[-33 \%,-18 \%,-8 \%,-1 \%]$ for $E_{\text {asym }}$ and $[-14 \%,-5 \%,-0 \%,+3 \%]$ for $E_{\text {asym }}$ for $\theta_{\mathrm{IMF}}=\left[90^{\circ}, 120^{\circ}, 150^{\circ}, 180^{\circ}\right]$, respectively. For both comparisons, there is a trend that the percent error gets larger for smaller $\theta_{\mathrm{IMF}}$; the dependence is described fairly well as the percent error being proportional to $-\cos \theta_{\mathrm{IMF}}$. This suggests that there is a systematic 


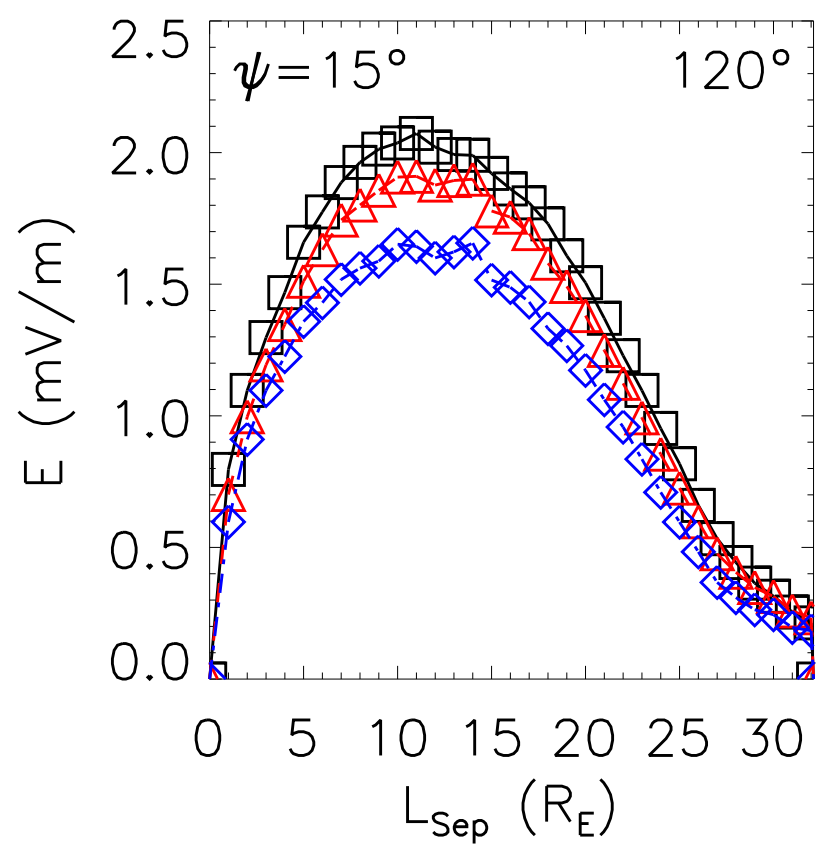

Figure 5.5: Comparison between the measured reconnection rate at the separator with the theoretical asymmetric reconnection rates in a simulation with $\theta_{\mathrm{IMF}}=120^{\circ}$ and positive dipole tilt of $\psi=15^{\circ}$. See caption of Fig. 5.4 for definitions.

effect causing an offset that increases with decreasing $\theta_{\mathrm{IMF}}$, such as our algorithm to find $\delta$ or $L$, but the relatively flat percent error with separator location suggests the scaling result is accurately predicting the dependence on plasma parameters local to the reconnection site for these simulations.

We note that the two curves for $E_{\eta, a s y m}$ and $E_{\text {asym }}$ should ideally lie on top of each other. However, in these cases there is some offset between the two. The percent difference between the two, using a form similar to Eq. (5.7), gives $[-22 \%,-13 \%,-8 \%,-8 \%]$ for the average percent error between the two theoretical predictions. Thus, $E_{\text {asym }}$ is systematically $10-20 \%$ less than $E_{\eta, \text { asym }}$ for these simulations.

We perform a similar comparison for a simulation that includes a positive dipole tilt $\psi=15^{\circ}$ (northern geomagnetic pole oriented sunward), with $\theta_{\mathrm{IMF}}=120^{\circ}$ and all solar wind parameters the same as our base simulations. Figure 5.5 displays the results from the 
dipole tilt simulation as before. We again see very good agreement in the scaling sense for both theoretical reconnection rates. As we did previously, we calculate the average percent differences for the middle third of the separator, for which the percent error is relatively constant. One finds the errors in $E_{\text {asym }}$ and $E_{\eta, \text { asym }}$ to be $-21 \%$ and $-7 \%$, respectively; these average percent differences are comparable to those seen in our base simulations for the same IMF clock angle. This suggests that the prediction is equally successful with or without a dipole tilt.

The results of this test are particularly important because our base simulations employ a high degree of symmetry given the solar wind's Earthward flow, and the IMF is further confined to the $y$ - $z$ GSM plane. The inclusion of the dipole tilt breaks this symmetry and causes the separator to move southward of the subsolar point and to be located in a region where the draping of the magnetosheath magnetic field across the magnetopause is noticeable. We find the magnetosheath's reconnecting magnetic field component near the subsolar magnetopause decreases by $7 \%$ in the dipole tilt simulation, suggesting draping is playing a role.

Finally, we perform an additional parametric test of the theory: we lower the IMF strength to 5 and $2 \mathrm{nT}$ in two separate simulations, each with $\theta_{\mathrm{IMF}}=120^{\circ}$ and all other solar wind parameters equal to those of our base simulations. Figure 5.6 displays the results from these simulations with the reconnection rates displayed as before for the simulation with (a) $B_{\mathrm{IMF}}=5$ and (b) $2 \mathrm{nT}$.

Before discussing the reconnection rates, we note one important difference among our $\theta_{\mathrm{IMF}}=120^{\circ}$ simulations without a dipole tilt. The total length of the dayside separator $L_{\text {Sep,tot }}$ increases as IMF strength is lowered: $L_{\text {Sep,tot }} \approx 33 \mathrm{R}_{\mathrm{E}}$ for our base simulation with $B_{\mathrm{IMF}}=20 \mathrm{nT}$ as seen in Fig. 5.4(b), whereas $L_{S e p, t o t} \approx 35 \mathrm{R}_{\mathrm{E}}$ and $L_{S e p, t o t} \approx 45 \mathrm{R}_{\mathrm{E}}$ for 

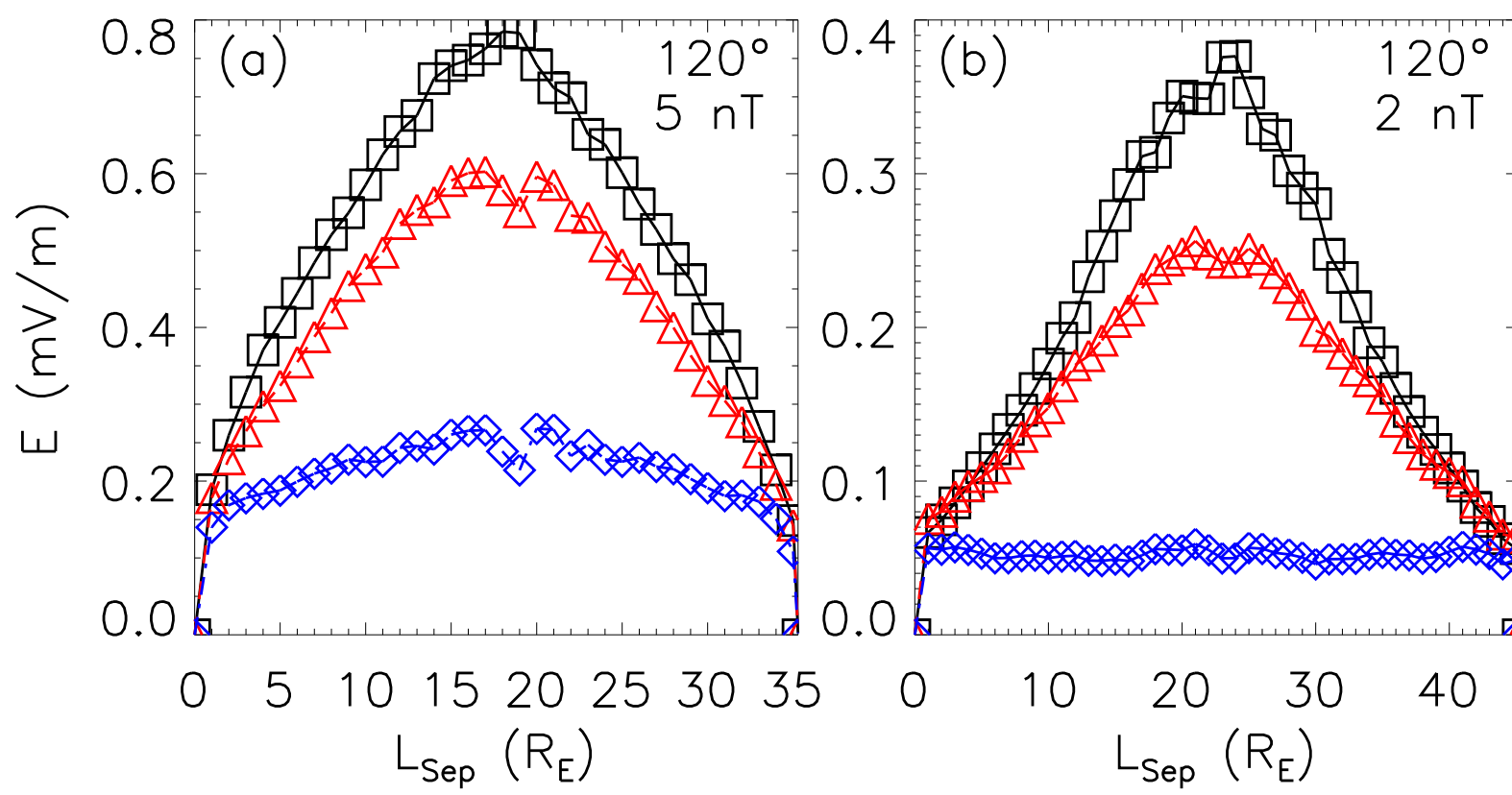

Figure 5.6: Comparison between the measured reconnection rate at the separator with theoretical asymmetric reconnection rates in distinct simulations with $\theta_{\mathrm{IMF}}=120^{\circ}$ and IMF strength of (a) 5 and (b) 2 nT. See the caption of Fig. 5.4 for definitions.

$B_{\mathrm{IMF}}=5$ and $2 \mathrm{nT}$, respectively, as seen in Fig. 5.6(a) and (b). The difference in separator lengths among these simulations results from pressure balance at the magnetopause, as we motivated in Section 4.2.1. The magnetic pressure of the IMF has a small contribution to the solar wind's total pressure so a decrease in the IMF strength lowers the solar wind's total pressure. The magnetopause correspondingly balloons outward resulting in a lengthening of the dayside separator.

As clearly seen in Fig. 5.6, the scaling arguments of asymmetric reconnection theory do much worse at predicting the reconnection rate measured at the separator. For the simulation with $B_{\mathrm{IMF}}=5 \mathrm{nT}$ simulation, the average percent errors of the middle third of the dayside separator in $E_{a s y m}$ and $E_{\eta, a s y m}$ are $-65 \%$ and $-21 \%$; likewise for the $B_{\mathrm{IMF}}=2 \mathrm{nT}$ simulation, the errors in $E_{\text {asym }}$ and $E_{\eta, a s y m}$ are $-84 \%$ and $-28 \%$, respectively. Secondly, $E_{\eta, a s y m}$ appears to have the best scaling with $E_{z^{\prime}}$, whereas $E_{a s y m}$ shows limited scaling agreement in the $5 \mathrm{nT}$ 

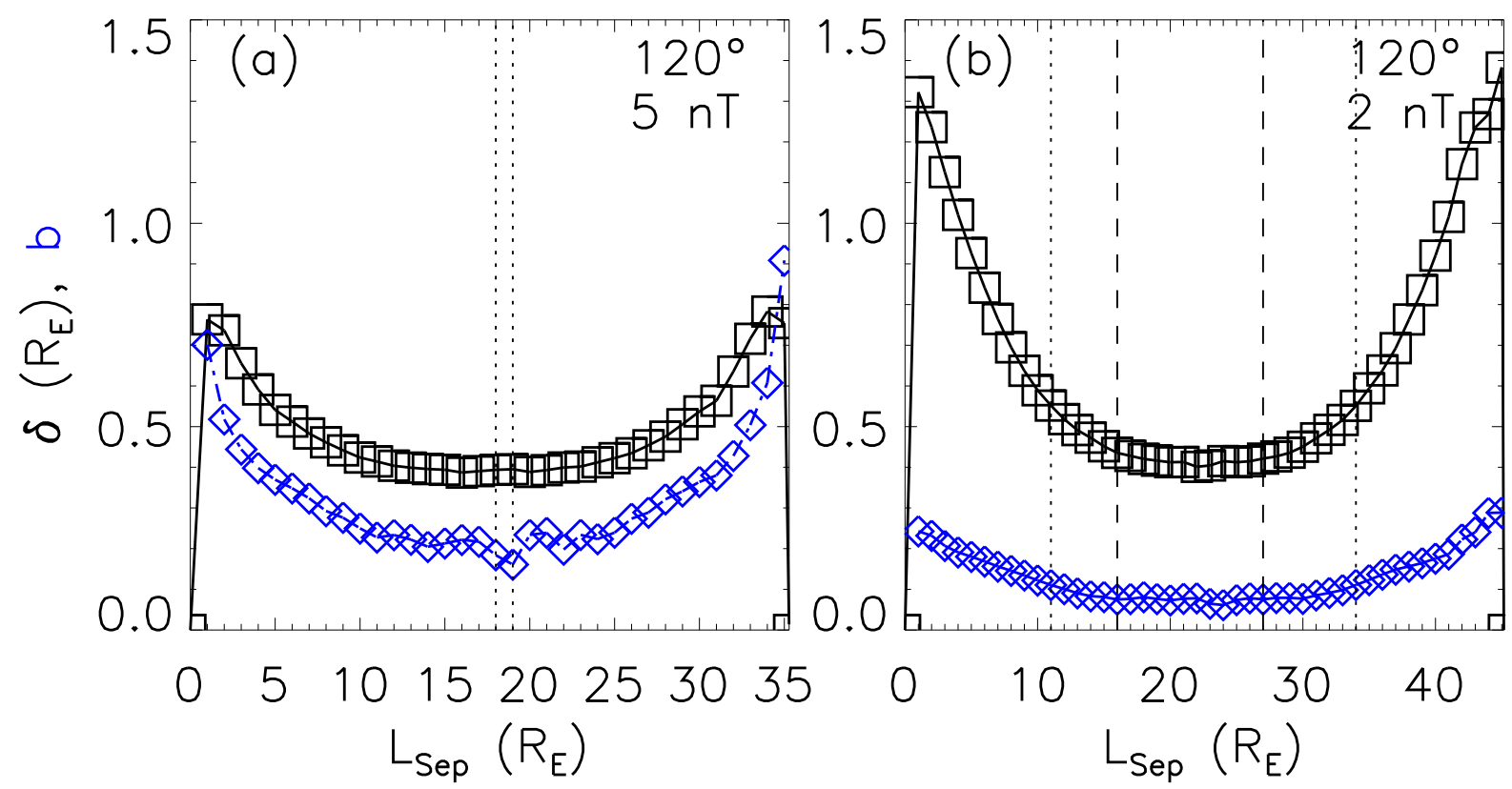

Figure 5.7: Plot of the dissipation region's half-width $\delta\left(\mathrm{R}_{\mathrm{E}}\right.$; black $\left.\square\right)$ and the magnetic field ratio $b$ (unitless; blue $\diamond$ ) as a function of distance along the separator measured from the northern null in $\mathrm{R}_{\mathrm{E}}$ in simulations with $\theta_{\mathrm{IMF}}=120^{\circ}$ and (a) $B_{\mathrm{IMF}}=5$ and (b) $2 \mathrm{nT}$. The resolution threshold condition of Eq. (5.13) is plot as vertical lines for simulation grid sizes of $\Delta=1 / 8$ (dotted) and $1 / 16 \mathrm{R}_{\mathrm{E}}$ (dashed); reconnection is sufficiently resolved for locations on the separator external to these vertical lines.

simulation and no scaling agreement is present for the $2 \mathrm{nT}$ simulation.

We argue that the reason for the lack of agreement in these cases results not from a failure in the theory, but rather from a numerical limitation of the present simulation grid, as previously noted in Cassak and Shay (2008). To demonstrate this, Fig. 5.7 plots the dissipation region's half-width $\delta$ in $\mathrm{R}_{\mathrm{E}}$ as black squares and the magnetic field ratio $b \equiv$ $B_{x^{\prime}, \mathrm{SH}} / B_{x^{\prime}, \mathrm{MS}}$ as blue diamonds as a function of the distance along the separator in $\mathrm{R}_{\mathrm{E}}$ as measured from the northern null in the simulations with IMF strength (a) 5 and (b) 2 nT.

One feature stands out in this figure: the magnetic field ratio $b$ is not constant along the magnetic separator. This has significant implications for the location of the separator within the magnetopause current layer across the dayside magnetopause. The location of the X-line within the dissipation region in terms of the magnetic field ratio $b$ was given by Eq. (1.18); 
we reprint this in the separator plane's coordinate system as

$$
\frac{\delta_{X, \mathrm{SH}}}{\delta_{X, \mathrm{MS}}} \sim \frac{B_{x^{\prime}, \mathrm{SH}}}{B_{x^{\prime}, \mathrm{MS}}} \equiv b,
$$

where $\delta_{X, \mathrm{SH}}$ and $\delta_{X, \mathrm{MS}}$ are the distances from the dissipation region's magnetosheath and magnetospheric edges to the separator, respectively. Specifically, the separator is located towards the magnetosheath for these simulations since $b<1$. For the reconnection dynamics to be properly resolved in the simulations, the distance from the dissipation region's magnetosheath edge to the separator $\delta_{X, \mathrm{SH}}$ must be resolved with one simulation grid cell of size $\Delta$ so that

$$
\delta_{X, \mathrm{SH}}>\Delta
$$

From Fig. 1.9, one finds that the dissipation region's full-width $2 \delta$ is defined as

$$
2 \delta=\delta_{X, \mathrm{SH}}+\delta_{X, \mathrm{MS}}
$$

Using these two equations, one can solve for $\delta_{X, \mathrm{SH}}$ which is

$$
\delta_{X, \mathrm{SH}}=2 \delta \frac{b}{b+1} .
$$

Using this result in Eq. (5.10) gives the minimum resolution required to resolve the separator's distance from the magnetosheath with one grid cell and is given by

$$
2 \delta \frac{b}{b+1}>\Delta
$$

Figure 5.7 displays the threshold condition given by Eq. (5.13) as vertical lines for grid 
cell sizes $\Delta=1 / 8$ (dotted), which is the grid size we use to resolve the dayside magnetopause and $1 / 16 \mathrm{R}_{\mathrm{E}}$ (dashed); locations along the separator which satisfy Eq. (5.13) lie exterior to these vertical lines. This implies that we are sufficiently resolving the reconnection dynamics only at locations (a) $L_{S e p}<18$ and $L_{S e p}>19 \mathrm{R}_{\mathrm{E}}$ and (b) $L_{S e p}<11$ and $L_{S e p}>34 \mathrm{R}_{\mathrm{E}}$. The region between the vertical lines displayed in Fig. 5.7 is consistent with the regions where we see the largest discrepancies between the measured reconnection rate and the theoretical predictions, as displayed in Fig. 5.6.

These tests therefore suggest that we are not properly resolving reconnection for portions of the separator at the dayside magnetopause for our lower $B_{\mathrm{IMF}}$ simulations and therefore these simulations are not appropriate for testing the theory. For future work, this can be further tested by doubling the simulation resolution to $\Delta=1 / 16 \mathrm{R}_{\mathrm{E}}$ in the simulation with $B_{\mathrm{IMF}}=5 \mathrm{nT}$. Another way of testing this hypothesis is to increase the explicit resistivity described in Chapter 2.2.2, since this causes the magnetopause current layer to broaden from magnetic diffusion. Another option is to perform a simulation with an IMF strength around $10 \mathrm{nT}$, which may be enough to sufficiently resolve reconnection along the entire separator. Nonetheless, some combination of these approaches is required to test the effect

of grid resolution. If the resolution proves to be the cause of the disagreement, this has important implications for other researchers studying dayside reconnection as many authors use numerical dissipation, so their dissipation region is under-resolved.

\subsection{Summary}

The analysis presented here suggests that, up to a scaling factor, the 2D asymmetric reconnection theory predicts the reconnection rate at the dayside magnetopause as a function of 
the upstream parameters local to the magnetic separator for southward IMF orientations. The theory has also been confirmed in previous scaling analyses of asymmetric reconnection in: laboratory experiments (Yoo et al., 2014; Rosenberg et al., 2015); observations of reconnection at Earth's dayside magnetopause (Mozer and Hull, 2010); anti-parallel reconnection in 2D slab geometries in resistive MHD (Cassak and Shay, 2007), two fluid (Hall-MHD with electron inertia) (Cassak and Shay, 2009), and particle-in-cell (Malakit et al., 2010; Hesse et al., 2013) simulations; and at the subsolar magnetopause for southward IMF in global simulations (Borovsky et al., 2008; Ouellette et al., 2014).

To the best of our knowledge, the present study is the only one of its kind to analyze reconnection local to the separator for oblique IMF orientations and including a dipole tilt. The present results suggest that the approach taken is appropriate for analyzing reconnection local to the magnetic separators. This suggests that we are uniquely poised to bridge the gap between reconnection physics local to the separator and reconnection's consequences for global magnetospheric dynamics. 


\section{Chapter 6}

\section{Conclusions}

\subsection{Summary of Results}

\subsubsection{Identifying Magnetic Separators in Global Magnetospheric Simulations}

In summary, we present a simple, efficient, and accurate method of tracing magnetic separators in global magnetospheric simulations with arbitrary solar wind conditions [Chapter 3 and Komar et al. (2013)]. Magnetic separators occur where magnetic fields of different topology meet, and are likely locations for where magnetic reconnection occurs. The method is to start at a magnetic null and iteratively trace the dayside separator by calculating the magnetic topology on the surface of spherical shells to locate regions of topological merging. We verify the method using a simple vacuum superposition magnetic field model with exact solutions for the separators. The technique improves on previous ones by being efficient, is good to arbitrary accuracy, and works for any solar wind condition.

We then trace separators in several distinct resistive global MHD simulations with $\theta_{\mathrm{IMF}}$ 
ranging from $0^{\circ}$ to $180^{\circ}$. The resulting magnetic nulls and separators in MHD are compared to those in vacuum superposition. We find that the $y$ - and $z$-coordinates of the magnetic nulls display similar qualitative trends in both models, but the migration of the null's $x$-coordinate in MHD is not captured by the vacuum superposition fields. We find that the method

described here can trace magnetospheric separators for arbitrary clock angle, whereas the last closed field line on the sun-Earth line only works for northward IMF in our simulations. Magnetospheric separators maintain a similar shape regardless of IMF clock angle and a large portion of the separators are approximately coplanar, although this plane does not contain the nulls. We find that both models have separators that change orientation without appreciably changing shape, but trends between models differ significantly. A preliminary test of the separator's dependence on solar wind number density $n$ reveals that the null locations and separator location do depend on number density, but separator orientation does not strongly depend on number density for our chosen solar wind parameters.

\subsubsection{Comparative Analysis of Dayside Reconnection Models}

With the ability to trace separators, we calculate the predictions of several magnetic reconnection location models at the dayside magnetopause in global resistive MHD simulations [Chapter 4 and Komar et al. (2015)]. Models tested are: the maximum magnetic shear model (Trattner et al., 2007), maximization of the asymmetric reconnection outflow speed (Swisdak and Drake, 2007), maximization of the asymmetric reconnection rate [Shay, private communication, 2009; Borovsky (2013)], the angle of bisection (Moore et al., 2002; Borovsky, 2008; Hesse et al., 2013), the maximization of the current density magnitude (Alexeev et al., 1998), and component reconnection [uniform out-of-plane (guide) magnetic field $\left(B_{M}\right)$ component] (Sonnerup, 1974; Gonzalez and Mozer, 1974). The first five models predict 
reconnection to occur at locations where a given parameter is maximized. We employ robust image processing techniques to determine each model's prediction. Each model is tested in separate global magnetospheric simulations with various IMF orientations, IMF strengths, and with a dipole tilt. The predictions for each model are then compared to the magnetic separators to determine which model, if any, accurately predicts the location of reconnection at the dayside magnetopause. We summarize our findings:

1. For the chosen simulation parameters, we find that all models are within a few Earth radii $\left(R_{E}\right)$ of the magnetic separators when the IMF has a southward orientation and no dipole tilt.

2. None of the models chosen for this study faithfully reproduce the entire magnetic separators when the IMF has a northward orientation and no dipole tilt. However, only the maximum magnetic shear model faithfully reproduces the portion of the separator nightward of the magnetic nulls where reconnection is expected to occur.

3. The asymmetric outflow speed and asymmetric Sweet-Parker reconnection rate more closely map the magnetic separators than other models tested under southward IMF orientations and no dipole tilt. The maximum magnetic shear model has a fixed orientation at the subsolar magnetopause and does not rotate with the magnetic separators for different IMF clock angles in simulations without a dipole tilt.

4. As the IMF strength is varied with fixed clock angle, the magnetic separators display different orientations than the predictions of the magnetic shear angle, and the asymmetric outflow speed and Sweet-Parker reconnection rate models.

5. In simulations with a dipole tilt of $+15^{\circ}$, we find most of the models map the magnetic 
separators when the IMF clock angle is $120^{\circ}$. However, none of the models chosen for this study faithfully reproduce the entire magnetic separator for a northward IMF orientation of $30^{\circ}$. Again, only the maximum magnetic shear model traces the portion of the separator nightward of the magnetic nulls. The dayside portion of the magnetic separator in this simulation moves duskward by approximately $45^{\circ}$ in longitude, as measured from the $+x_{\mathrm{GSM}}$ axis.

6. One attempt to account for flow shear parallel to the reconnecting components of the magnetosheath and magnetospheric magnetic fields improves the performance of the asymmetric outflow speed and asymmetric reconnection rate models under northward IMF orientations. Predictions for southward IMF orientations are mostly unaffected. However, the way flow shear is incorporated in the present work is now known to likely be incorrect.

\subsubsection{Local Analysis of Reconnection at the Dayside Magnetopause}

We detail a systematic approach to quantify reconnection local to magnetic separators arising in global magnetospheric simulations [Chapter 5]. We find the reconnection planes normal to magnetic separator locations, and use them to measure the upstream plasma parameters from the magnetic separator in order to compare local asymmetric reconnection theory predictions with the reconnection rate at the separator. We test the theory in distinct simulations with southward IMF orientations for our base simulation parameters, a simulation with a dipole tilt, and two simulations where the IMF strength is lowered significantly from that of our base simulation parameters. With the notable exceptions of simulations with IMF strengths lower than our base simulation parameters, local asymmetric theory works surprisingly well. 
For the two simulations with lower IMF strength, it is found that the theory does not work as well in predicting the measured reconnection rate, but we argue that this is not necessarily a failure of the local asymmetric reconnection theory, but rather results from numerical effects where the sub-dissipation region scale reconnection dynamics are not properly resolved with the specified simulation grid.

\subsection{Limitations to the Present Work}

The present study employed a few underlying assumptions. For the global magnetospheric simulations, we employ a uniform, explicit resistivity even though Earth's magnetopause is essentially collisionless; this choice ensures our base simulations are well resolved, while reducing the likelihood of Flux Transfer Events (FTEs) (Russell and Elphic, 1978) in our simulations. However, recent advances have been made to trace magnetic separators in simulation when FTEs are present (Glocer et al., 2015).

Most of the reconnection location models discussed in Chapter 4 are independent of dissipation mechanism, so the results on the validity of each model therein should be independent of our choice to include an explicit resistivity. However, it is unknown if the magnetic separators depend on dissipation mechanism. Future work will need to extend the present study to global Hall-MHD, hybrid, and/or kinetic simulations.

The present research detailed in this dissertation uses idealized solar wind conditions with a few limitations not wholly representative of solar wind observations. The present work has ignored the effect of including an IMF $B_{x}$ component. Previous studies found that under southward IMF orientations, the reconnection site moves northward for $B_{x}>0$ and southward when $B_{x}<0$ (Peng et al., 2010; Hoilijoki et al., 2014). Additionally, we 


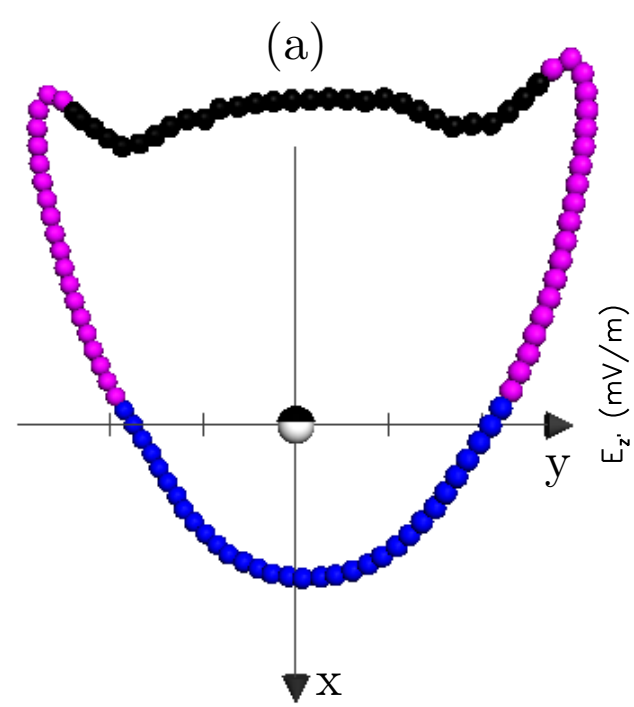

(b)

\section{Dayside Dawn Night Dusk}

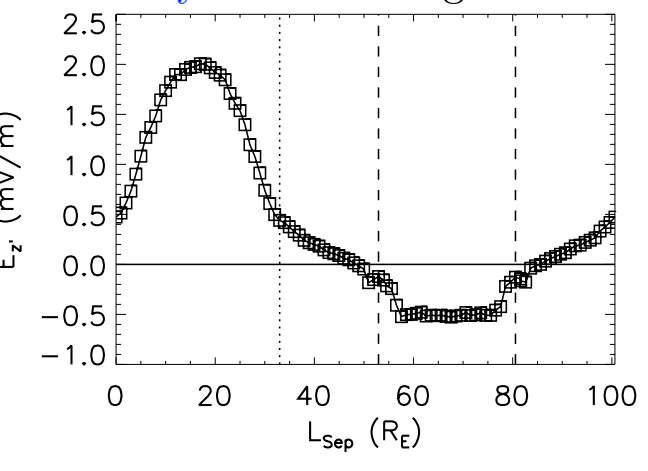

Figure 6.1: Plot of the (a) global separator looking down along the $-z_{\mathrm{GSM}}$ axis and (b) the parallel electric field $E_{z^{\prime}}$ as a function of distance along the separator from the northern null measured in $\mathrm{R}_{\mathrm{E}}$ in our base simulation with $\theta_{\mathrm{IMF}}=120^{\circ}$. The dayside separator discussed throughout this dissertation is depicted as the blue portion, the dawn and dusk portions nightward of the magnetic nulls are depicted in purple, and the portion of the magnetic separator through the magnetotail is colored black.

perform our analysis after the simulations have achieved a quasi-steady-state, which does not capture the magnetosphere's response to dynamic solar wind conditions (Laitinen et al., 2006, 2007). Understanding the response of Earth's magnetosphere for a broader range of solar wind conditions is of the utmost importance for realistic space weather forecasting, and will be the subject of future work.

The local analysis of reconnection presented here takes important first steps in analyzing reconnection local to the magnetic separator. However, this analysis limited its focus to the dayside portion of the magnetic separators. The separator extends toward the magnetotail where it forms a closed loop. The methodology here should work for locations extending further nightward of the magnetic nulls, like those found for northward IMF conditions described in Chapter 4.2.3, but further research is necessary.

To demonstrate this, Fig. 6.1(a) displays preliminary results from our base simulation 
with $\theta_{\mathrm{IMF}}=120^{\circ}$. The result is the composite of the iterative separator tracing technique discussed in Section 4.2.3 which starts at both southern and northern magnetic nulls and traces the separator nightward for $25 \mathrm{R}_{\mathrm{E}}$ resulting in the purple portions of the separator at the dawn $\left(y_{\mathrm{GSM}}<0\right)$ and dusk $\left(y_{\mathrm{GSM}}>0\right)$ flanks. The nightside portion of the separator is displayed in black and crosses through the magnetotail; this portion of the separator is traced by starting at the last point on the dawn flank and uses the last point on the dusk separator as its stopping point. The total length of the separator is $L_{S e p}=101.6 \mathrm{R}_{\mathrm{E}}$ as measured from the northern null along the separator and returning to the northern null. We will discuss future work that will be enabled by this type of study in the next section.

Another limitation is that we do not investigate the application of the local reconnection theory for northward IMF conditions. Preliminary results (not shown) suggest that the asymmetric reconnection theory performs well for the measured reconnection rate along the dayside separator in these simulations. We currently do not look at the magnetospheric cusps to determine the reconnection rate in this region, and will need to going forward. Recent observations show evidence for reconnection occurring near the subsolar magnetopause for northward IMF orientations. However, it is currently under debate as to how fast reconnection is at either location (K. Trattner and S. Fuselier, private communication, 2015).

Finally, the local analysis at present has ignored a few other effects that can alter the theoretical scaling of the reconnection rate at the magnetic separator. The first effect to account for is how asymmetries in the downstream plasma affect the reconnection rate $(Y a$ mada et al., 1997; Murphy et al., 2010). Away from the subsolar point in our simulations even without a dipole tilt, these asymmetries are observed. Interestingly, the asymmetric theory tested here does quite well despite ignoring these downstream asymmetries. However, preliminary results of the reconnection outflow speeds (not shown) suggest this effect will 
need to be accounted for in a full comparison with the theory. Another effect currently unaccounted for is the $\mathrm{X}$-line drift and related suppression of reconnection resulting from flow shear parallel to the reconnecting magnetic field components at the magnetic separator (Doss et al., in prep.). There is likely to be flow parallel to the reconnecting magnetic field components local to the separator for reconnection near the cusps during periods with northward IMF, so this effect must be included in the present analysis. The present approach assumes that the $B_{z^{\prime}}$ component does not affect the reconnection rate, which may not be the case. In particular, the out-of-plane component plays a key role in setting up a diamagnetic drift in the outflow direction (Swisdak et al., 2003, 2010; Beidler and Cassak, 2011). The theory does not include diamagnetic effects, nor is it present in resistive MHD, and this effect is known to alter the reconnection rate. These three effects can all be present at the magnetopause, but it is presently unclear how to combine their effects and will be the subject of future work.

\subsection{Future Work}

The development of the techniques presented in this dissertation enables one to locate magnetic separators in global magnetospheric simulations and then locally analyze reconnection at these separators. This presents a major advancement in the ability to address the longstanding question of whether magnetic reconnection is a local or global magnetospheric process.

To see an example of how the present work will help address the question about local versus global control of dayside reconnection, Fig. 6.1(b) shows the measured reconnection rate $E_{z^{\prime}}$ as a function of $L_{S e p}$ for the full separator in Fig. 6.1(a); $\hat{\mathbf{z}}^{\prime}$ is the same as given 
by Eq. (5.1). Recall $E_{z^{\prime}}$ is the integrand of Eq. (1.28), resulting in a reconnection potential along the entire separator $\Delta \Phi_{S e p}=252.4 \mathrm{kV}$; for comparison the cross polar cap potential given by Eq. (1.4) is $\Delta \Phi_{P C}=128.4 \mathrm{kV}$. In future work, we will use the separators to compare measures of local and global reconnection.

A noteworthy feature seen in $E_{z^{\prime}}$ is that it transitions from positive to negative as the separator is traversed from the dawn portion to the night portion; likewise, a negative to positive transition of $E_{z^{\prime}}$ can be seen upon crossing the nightside to dusk portion. This is suggestive of an observational signature for dayside reconnection, i.e., IMF + Geomagnetic Field $\Rightarrow 2$ Open Fields occurs where $E_{z^{\prime}}>0$. Conversely, nightside reconnection, i.e., 2 Open Fields $\Rightarrow$ IMF + Geomagnetic Field is likely occurring where $E_{z^{\prime}}<0$. From this signature in the parallel electric field, we can tell where magnetic fields that will undergo dayside reconnection encounter the magnetic separator. In future work, we will convect the magnetic fields back to the solar wind to determine a length in the solar wind, inside of which all IMF lines will make contact with the separator and undergo reconnection. (Those that argue for reconnection as a global process refer to this as the geoeffective length.) Previous attempts to study this have used indirect methods to identify the geoeffective length, so being able to precisely define this length will allow for its parametrization for arbitrary solar wind conditions, and to assess how this length may or may not contribute to the global reconnection process.

We previously noted that a limitation of the research detailed here is the inclusion of an explicit resistivity whereby reconnection is mediated by collisions between ions and electrons. Earth's magnetosphere is not collisional, so collisionless mechanisms can play an important role for reconnection at Earth. In fact, collisionless mechanisms fundamentally alter reconnection, where local reconnection rates of collisionless reconnection are much faster than 
that of collisional reconnection (Birn et al., 2001). Incorporating collisionless effects into the MHD model requires modification of the electric field $\mathbf{E}$ and takes the form of the generalized Ohm's law given by

$$
\mathbf{E}=-\mathbf{u} \times \mathbf{B}+\eta \mathbf{J}+\frac{\mathbf{J} \times \mathbf{B}}{e n}-\frac{1}{e n} \nabla \cdot \mathbf{P}_{e}+\frac{m_{e}}{e^{2}} \frac{d(\mathbf{J} / n)}{d t}
$$

where $e, n, \mathbf{P}_{e}$, and $m_{e}$ are the electron's charge, density, pressure tensor, and mass, respectively. The first and second terms on the right hand side are the convective and resistive electric fields present in resistive MHD as discussed in Chapter 2.1, the third is the Hall term, the fourth is the electron pressure term, and the fifth term is the electron inertia term.

The Hall term was recently shown to significantly alter the global dynamics at Jupiter's moon Ganymede, with effects not seen in resistive MHD (Dorelli et al., 2015). Magnetic separators arising in the Ganymede Hall-MHD simulations were identified, but were ultimately limited in their use for measuring the Hall reconnection rate (A. Glocer, private communication, 2014). This results from a lack of a parallel reconnection electric field from the Hall term since $\mathbf{E}_{\text {Hall }} \propto \mathbf{J} \times \mathbf{B}$. The results of the present analysis suggest that one can estimate the Hall electric field parallel to the separator by measuring the upstream plasma parameters and calculating the generalized reconnection rate $E_{\text {asym }}$. This will allow us to assess how changes to the local reconnection physics affect the global reconnection process, and will be the subject of future work.

The ultimate goal of this line of research is a predictive capability for the magnetospheric response to given solar wind input. Ideally, given measurements upstream from the Earth in the solar wind, we will be able to predict how the plasma is altered at the bow shock and through the magnetosheath until it reaches the magnetopause. One would like to use these 
parameters to predict how the dayside magnetosphere responds. The present work goes a long way towards the latter capability. Once this dayside parametrization is done, one can further understand the magnetospheric response by incorporating physical models of other magnetospheric domains such as the plasmasphere, radiation belts, and ring current. This ultimately aids the laudable goal in developing a system-level understanding required for predicting the global magnetospheric dynamics resulting from the magnetosphere's interaction with the solar wind (Borovsky et al., 2013a). 


\section{Appendix A}

\section{List of CCMC Simulations}

All of the following runs were requested from NASA's Community Coordinated Modeling Center using their Runs on Request service, http://ccmc.gsfc.nasa.gov/requests/requests.php. The runs are searchable under the heading "Global Magnetosphere Models Results" from the View Results tab at http://ccmc.gsfc.nasa.gov/results/index.php and searching for SWMF runs submitted by Colin Komar. The runs listed below were analyzed to produce the results included in this dissertation. 
Table A.1: Chapter 3 simulations. Simulations use $0.125 \mathrm{R}_{\mathrm{E}}$ grid resolution in the region $-6<x<$ $10,-10<y<10,-5<z<5 \mathrm{R}_{\mathrm{E}}$, unless otherwise noted.

\begin{tabular}{ll}
\hline CCMC Run Number & Description \\
\hline Colin_Komar_030811_1 & Simulation with IMF clock angle 30 \\
Colin_Komar_071610_1 & Simulation with IMF clock angle $60^{\circ}$ \\
Colin_Komar_021111_1 & Simulation with IMF clock angle $90^{\circ}$ \\
Colin_Komar_011211_1 & Simulation with IMF clock angle $120^{\circ}$ \\
Colin_Komar_031511_1 & Simulation with IMF clock angle $150^{\circ}$ \\
Colin_Komar_051812_1 & Simulation with IMF clock angle $165^{\circ}$ \\
Colin_Komar_070110_1 & Simulation with due Southward IMF with explicit resistivity \\
Colin_Komar_070110_2 & Simulation with due Southward IMF without explicit resistivity \\
Colin_Komar_052312_1 & Simulation with IMF clock angle 30 and lowered solar wind \\
& number density $n_{S W}=5$ cm $^{-3}$ \\
Colin_Komar_101910_1 & Simulation with IMF clock angle $60^{\circ}$ and $0.125 \mathrm{R}_{\mathrm{E}}$ resolution \\
& domain $-6<x<10,-10<y<10,-12<z<12 \mathrm{R}_{\mathrm{E}}$ \\
Colin_Komar_012913_1 & Simulation with IMF clock angle $150^{\circ}$ and $0.125 \mathrm{R}_{\mathrm{E}}$ resolution \\
& domain $-6<x<10,-15<y<15,-10<z<10 \mathrm{R}_{\mathrm{E}}$ \\
Colin_Komar_012913_2 & $\begin{array}{l}\text { Simulation with IMF clock angle } 165^{\circ} \text { and } 0.125 \mathrm{R}_{\mathrm{E}} \text { resolution } \\
\text { domain }-6<x<10,-15<y<15,-10<z<10 \mathrm{R}_{\mathrm{E}}\end{array}$
\end{tabular}

Table A.2: Chapter 4 simulations. Simulations use $0.125 \mathrm{R}_{\mathrm{E}}$ grid resolution in the region $-15<x, y, z<$ $15 \mathrm{R}_{\mathrm{E}}$, unless otherwise noted.

\begin{tabular}{|c|c|}
\hline CCMC Run Number & Description \\
\hline Colin_Komar_110813_1 & Simulation with IMF clock angle $30^{\circ}$ \\
\hline Colin_Komar_101910_1 & $\begin{array}{l}\text { Simulation with IMF clock angle } 60^{\circ} \text { and } 0.125 \mathrm{R}_{\mathrm{E}} \text { resolution } \\
\text { domain }-6<x<10,-10<y<10,-12<z<12 \mathrm{R}_{\mathrm{E}}\end{array}$ \\
\hline Colin_Komar_110813_2 & Simulation with IMF clock angle $90^{\circ}$ \\
\hline Colin_Komar_110813_3 & Simulation with IMF clock angle $120^{\circ}$ \\
\hline Colin_Komar_012913_1 & $\begin{array}{l}\text { Simulation with IMF clock angle } 150^{\circ} \text { and } 0.125 \mathrm{R}_{\mathrm{E}} \text { resolution } \\
\text { domain }-6<x<10,-15<y<15,-10<z<10 \mathrm{R}_{\mathrm{E}}\end{array}$ \\
\hline Colin_Komar_012913_2 & $\begin{array}{l}\text { Simulation with IMF clock angle } 165^{\circ} \text { and } 0.125 \mathrm{R}_{\mathrm{E}} \text { resolution } \\
\text { domain }-6<x<10,-15<y<15,-10<z<10 \mathrm{R}_{\mathrm{E}}\end{array}$ \\
\hline Colin_Komar_121613_1 & Simulation with IMF clock angle $120^{\circ}$ and $B_{\mathrm{IMF}}=5 \mathrm{nT}$ \\
\hline Colin_Komar_121613_2 & Simulation with IMF clock angle $120^{\circ}$ and $B_{\mathrm{IMF}}=2 \mathrm{nT}$ \\
\hline Colin_Komar_121613_3 & Simulation with IMF clock angle $30^{\circ}$ and dipole tilt $\psi=15^{\circ}$ \\
\hline Colin_Komar_121613_4 & Simulation with IMF clock angle $120^{\circ}$ and dipole tilt $\psi=15^{\circ}$ \\
\hline
\end{tabular}


Table A.3: Chapter 5 simulations. Simulations use $0.125 \mathrm{R}_{\mathrm{E}}$ grid resolution in the region $-15<x, y, z<$ $15 \mathrm{R}_{\mathrm{E}}$, unless otherwise noted.

\begin{tabular}{|c|c|}
\hline CCMC Run Number & Description \\
\hline Colin_Komar_110813_2 & Simulation with IMF clock angle $90^{\circ}$ \\
\hline Colin_Komar_110813_3 & Simulation with IMF clock angle $120^{\circ}$ \\
\hline Colin_Komar_012913_1 & $\begin{array}{l}\text { Simulation with IMF clock angle } 150^{\circ} \text { and } 0.125 \mathrm{R}_{\mathrm{E}} \text { resolution } \\
\text { domain }-6<x<10,-15<y<15,-10<z<10 \mathrm{R}_{\mathrm{E}}\end{array}$ \\
\hline Colin_Komar_011315_1 & Simulation with IMF clock angle $180^{\circ}$ \\
\hline Colin_Komar_121613_4 & Simulation with IMF clock angle $120^{\circ}$ and dipole tilt $\psi=15^{\circ}$ \\
\hline Colin_Komar_121613_1 & Simulation with IMF clock angle $120^{\circ}$ and $B_{\mathrm{IMF}}=5 \mathrm{nT}$ \\
\hline Colin_Komar_121613_2 & Simulation with IMF clock angle $120^{\circ}$ and $B_{\mathrm{IMF}}=2 \mathrm{nT}$ \\
\hline
\end{tabular}




\section{Appendix B}

\section{Overview of the BATS-R-US}

\section{Simulation Code}

This chapter serves as a brief overview of the Block Adaptive Tree Solarwind Roe-type Upwind Scheme (BATS-R-US) MHD code developed at University of Michigan and used in this study. BATS-R-US solves the conservative form of the resistive MHD equations, as presented in Chapter 2, on a three-dimensional, rectangular, irregular grid in order to simulate the global magnetosphere (Powell et al., 1999; Gombosi et al., 2000; De Zeeuw et al., 2000). BATS-R-US is just one component of the Space Weather Modeling Framework [SWMF] ( Tóth et al., 2005; Tóth et al., 2012), which contains several codes modeling different regions of Earth's magnetosphere and beyond, including the radiation belts, ring current, and ionosphere. Each code passes state variables (magnetic fields, densities, etc.) to other codes in order to simulate the influences of one magnetospheric region upon another.

We now describe how the BATS-R-US code works by explaining the meaning of the letters in its name. The simulation employs a hierarchical "tree" structure consisting of 
blocks. The 3D simulation grid is broken up into a number of specified master blocks and is further subdivided into "mother" blocks which contain their smaller "daughter" blocks on down to the smallest simulation grid size. Every block contains simulation grid cells all of one size, and moving up the tree goes to the next largest grid cell size. Extending the tree analogy to our high resolution grid has the region with resolution $1 / 8 R_{E}$ acting as the highest branch on the tree, whereas blocks in the distant magnetotail with $8 \mathrm{R}_{\mathrm{E}}$ serve as the trunk/roots of the tree. The code has the capability to adaptively coarsen or refine blocks depending on whether gradients in some plasma parameter are larger than some specified threshold, but we do not employ this feature in our simulations. The plasma variables inside each block are solved at the smallest time scale corresponding to the fastest MHD wave that can be resolved with the given block resolution. This results in different time scales for different sized resolution blocks. For the sake of computational efficiency, the code keeps a master clock at each simulation time step which is used to determine those blocks in need of updating should they lag begin the master clock.

The algorithm to update the conservative form of the MHD equations is the Roe approximate Riemann solver. The Riemann problem determines how discontinuities evolve in time. Here the problem arises at cell centers where variables are assumed to have a particular shape within cells. The variables can be discontinuous at cell centers. The Roe-solver gives an approximate Riemann solution by linearizing the flux across the interface.

Finally, the code employs an upwind finite differencing scheme to update plasma variables. The code determines the direction a parameter of interest flows in from to determine what direction to take its finite difference.

In concluding this section, we note that BATS-R-US has incorporated other physics not included in the resistive MHD model and can solve a version of the generalized Ohm's law, 
given by Eq. (6.1). This feature of the code will allow us (and others) to address the future projects mentioned in Chapter 6. The reader is referred to the description of the SWMF provided by Tóth et al. (2012) for a comprehensive discussion on the different physical models of BATS-R-US. 


\section{Appendix C}

\section{Setting up SWMF to Run on NASA's}

\section{Pleiades Supercomputer}

Below are instructions on how to compile and run the SWMF code on NASA's Pleiades Supercomputer. The version of the SWMF code is undergoing constant testing and updating, with the steps below working for more recent code versions.

The .login file in the user's home directory should load the following modules on Pleiades, which as of the writing of this dissertation allowed for the successful installation, compilation, execution, and analysis of the SWMF code:

- module load comp-intel/2013.1.117

- module load mpi-sgi/mpt.2.11r13

- module load idl

- module load tecplot

These modules will automatically load upon logging into Pleiades, but one can also type 
source .login

into the command prompt to immediately load these modules.

Go to the SWMF folder and start with a clean install by entering

Config.pl -uninstall

into the command prompt. Re-install the SWMF code with

Config.pl -install -compiler=ifort, icc -03

along with any additional flags as detailed in the SWMF Users Guide. (Note: One must use either the -03 or -04 flags to compile SWMF with the Intel compilers.)

After SWMF has finished compiling, one must edit the Makefile.conf file in the source code directory. Line 22 must be edited in order to successfully compile the code for the desired CPU architecture the code will be run on, as detailed on the NASA Pleiades Knowledge Base http://www.nas.nasa.gov/hecc/support/kb/?v=1. For example, editing Line 22 of Makefile.conf to

MPILIB $=$-openmp -Impi -axAVX -xSSE4.1

allows the code to run on Pleiades' Westmere, Ivy Bridge, and Sandy Bridge CPU types.

After following these steps, one may run the test suite detailed in the SWMF User Guide http://csem.engin.umich.edu/tools/swmf/documentation/HTML/SWMF/index.html, or begin running the code with the input files detailed in the appendices that follow. 


\section{Appendix D}

\section{SWMF Batch Script}

The script comes from a shell batch script entitled SWMF.run. It contains all the information to compile the SWMF code, run the job using the Portable Batch System (PBS) scheduling service, and additionally save the setup and output files to the archived backup.

To compile the code, enter the following into Pleiades' command line:

csh -vx SWMF.run init $>\&$ initRUN_ID

The commands above will output diagnostic information to the text file initRUN_ID. In particular, one is interested in the line

ifort -o/home6/ckomar/Master_Codes/SWMF/SWMF_20140110/bin/SWMF.exe swmf.o

$$
\text { Tmp_*/*.o -r8 -openmp -Impi -axAVX -xSSE4.1 }
$$

to check that there are no errors and that the code compiled successfully. Lastly, one will want to check the last several lines of the diagnostic text file to ensure that the code directory was successfully moved to to the user's \$NOBACKUP location.

To submit the job to the Pleiades queue one will type the following into the command line: 
qsub SWMF.run

The user will receive an e-mail upon the job's completion.

To save the files to the archived backup, one needs to input into the command line

csh -vx SWMF.run fin $>\&$ finRUN_ID

where diagnostic information is output to the text file finRUN_ID.

The vast majority of lines the user will need to change occur within the first 25 lines of SWMF.run. Variables include:

RUN_ID The run id for the simulation

N_NODES The number of processor nodes

N_CPUS The number of CPUs per node that will be used

N_MPI The number of MPI processes per node

WALLTIME The estimated time that the simulation will take to complete given in hh:mm:ss

PID The PI's project id

CODE_DIR The filepath to the SWMF code installation

DR The project directory

SVDIR The file path for the save directory on the archive backup

VERSION The SWMF components that the code will use

GRID The block size in number of cells, the maximum number of cells per block, and the maximum number of implicit cells per block, e.g., one block is $6 \times 6 \times 6$ cells, 400 blocks per processor, and a maximum of 100 implicit blocks per processor 
OPTIONS Specifies different options for the individual SWMF components

GRID_FILE The file path to the grid file that will be input to the PARAM.in file (Appendices $\mathrm{E}$ and $\mathrm{F}$ have more details)

(For more information on what can be input into VERSION and OPTIONS, the reader is referred to the SWMF User Guide.

http://csem.engin.umich.edu/tools/swmf/documentation/HTML/SWMF/index.html)

The batch script is as follows:

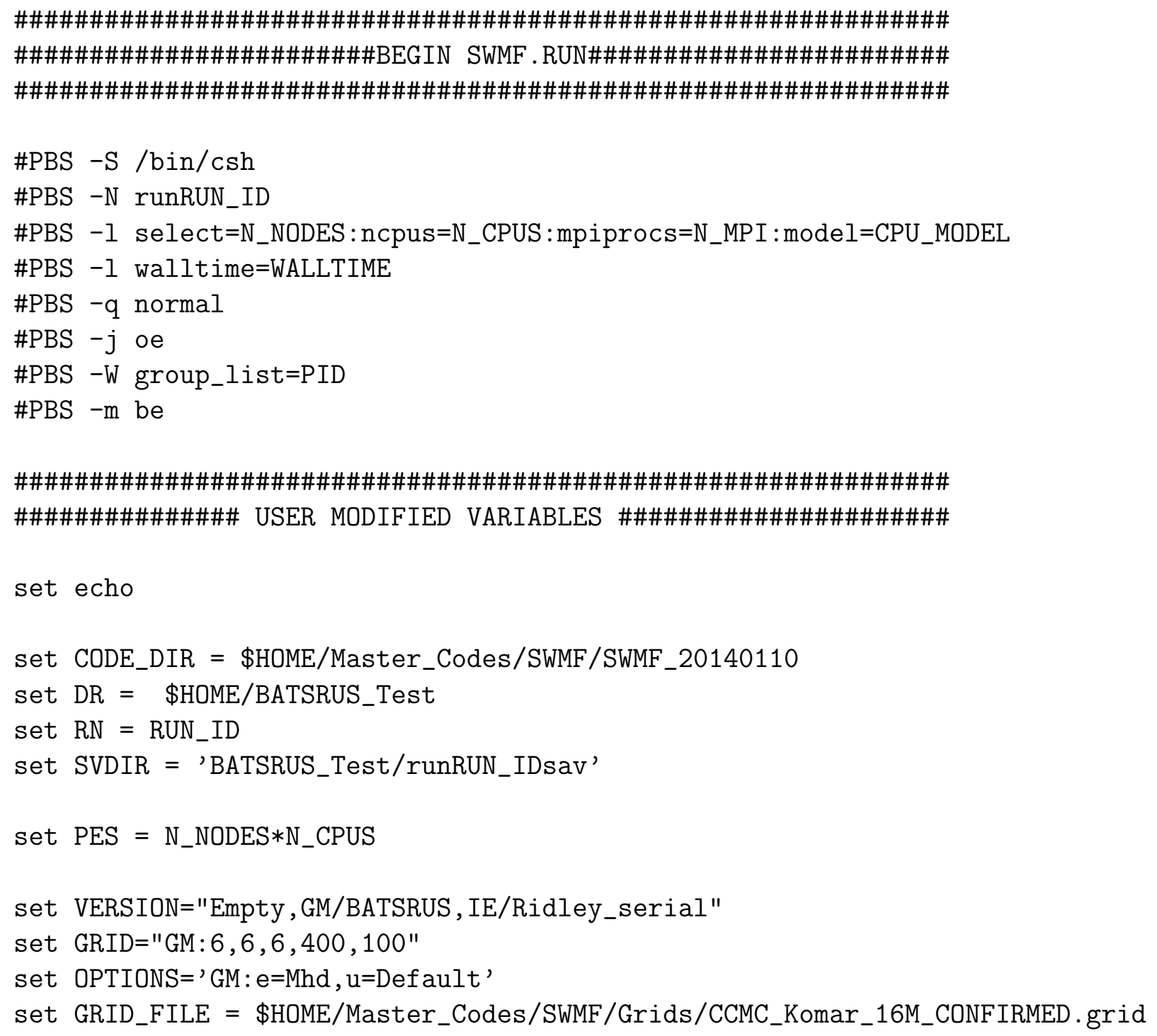




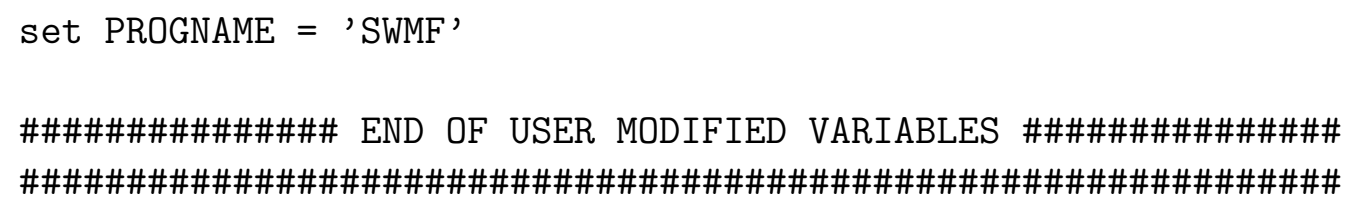




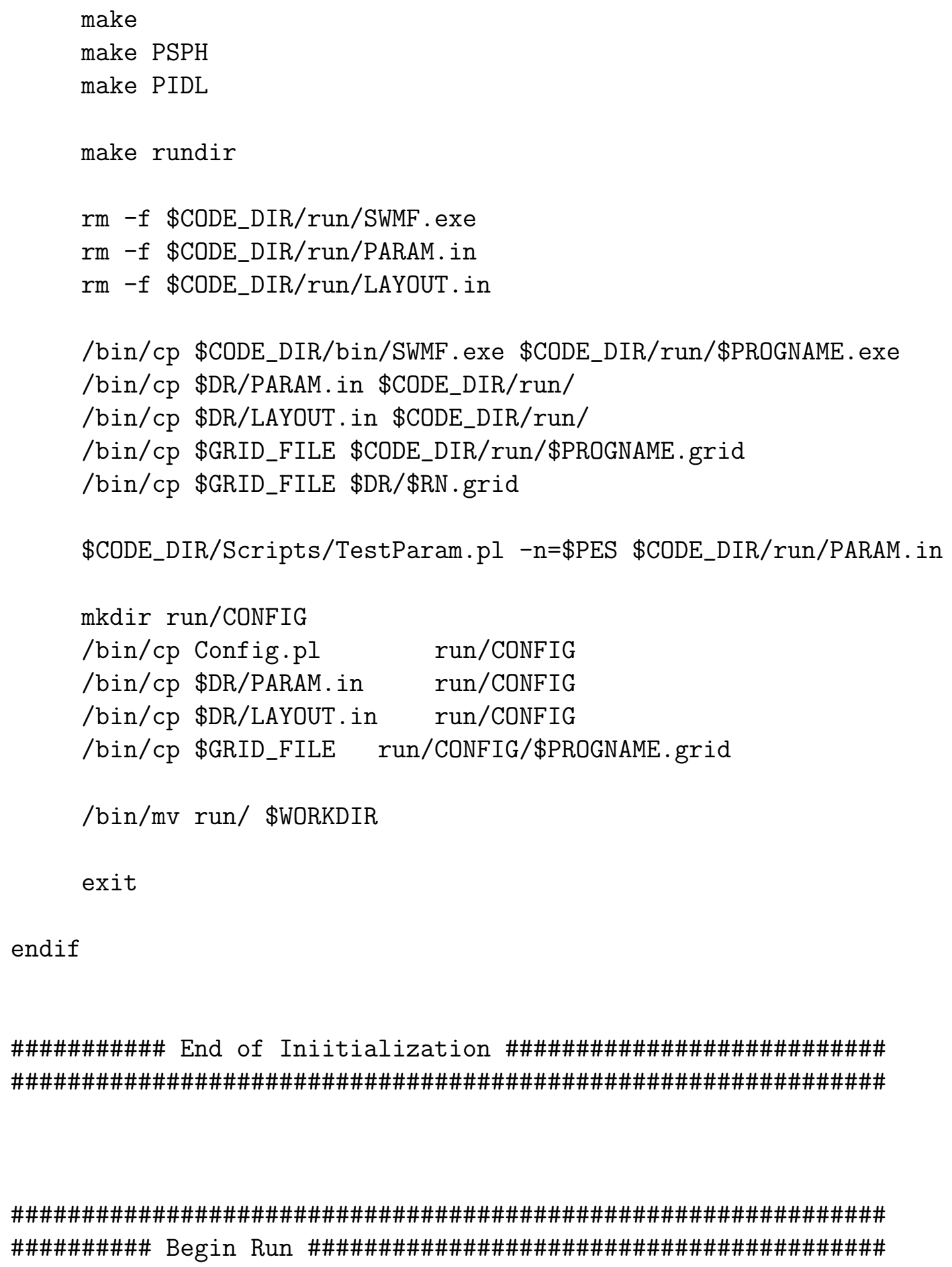


set WORKDIR $=$ 'cat $\mathrm{pwd} . \$ \mathrm{RN}$ '

cd \$WORKDIR

echo '\#\#\#\#\#\#\#\#\#\#\#\#\# RUNNING PROGRAM \#\#\#\#\#\#\#\#\#\#\#\#\#'

time mpiexec -np \$PES./\$PROGNAME. exe > log\$RN.out

echo '\#\#\#\#\#\#\#\#\#\#\#\#\#\# FINISHED PROGRAM \#\#\#\#\#\#\#\#\#\#\#\#\#'

PostProc.pl -n=\$PES -m -v - cat \$WORKDIR/RESULTS >\& PostProc $\$ R N$. log

/bin/cp $-r$ CONFIG RESULTS/.

/bin/cp PostProc\$RN.log \$DR/PostProc\$RN.log

$/ \mathrm{bin} / \mathrm{cp} \log \$ R N$.out $\$ \mathrm{DR} / \log \$ \mathrm{RN}$. out

/bin/cp \$PROGNAME.SUCCESS \$DR/run\$RN.SUCCESS

exit

endif

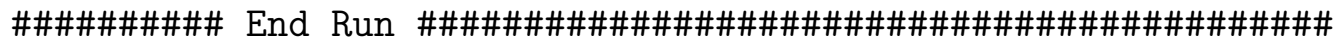

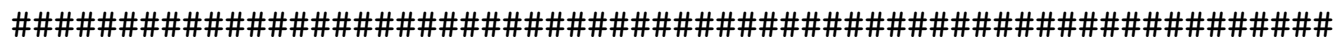

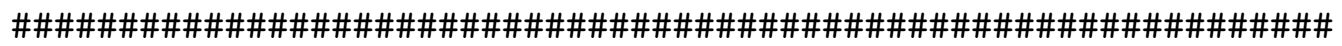
\#\#\#\#\#\#\#\#\# Finish up run: Save files to Archive \#\#\#\#\#\#\#\#\#\#\#\#\#

if ( $\$ 1==$ 'fin') then

echo $\$ 0$

cd $\$ D R$

set WORKDIR = 'cat pwd. $\$$ RN'

cd \$WORKDIR

tar -czvf \$WORKDIR/RESULTS.tar.gz \$WORKDIR/RESULTS

/usr/local/bin/shiftc -d \$WORKDIR/RESULTS.tar.gz lfe:\$SVDIR/.

exit

endif

\#\#\#\#\#\#\#\# End Finish up run: Save files to Archive \#\#\#\#\#\#\#\#\# 


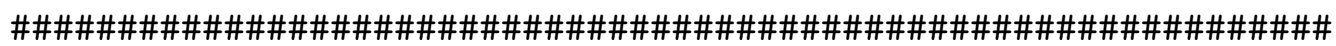




\section{Appendix E}

\section{SWMF PARAM.in}

The following code is from a PARAM.in file that specifies the setup for the individual SWMF Global Magnetosphere (GM) and Ionospheric Electrodynamics (IE) components, very similar to the setup used by NASA's CCMC. In addition, it takes input from a file SWMF.grid which specifies the grid resolution for the computational domain of the GM component, with a sample given in Appendix F. The reader is directed to the SWMF User's Guide http://csem.engin.umich.edu/tools/swmf/documentation/HTML/SWMF/index.html for information relating to each setup command and variable.

\#ECHO

$\mathrm{T}$

\#DESCRIPTION

Run GM to test grid customization

\#PLANET

EARTH

\#IDEALAXES

! align rotation and magnetic axes with $\mathrm{z}$ axis

\#ROTATION

F UseRotation

\#STARTTIME

2000 iYear 
01 iMonth

01 iDay

00 iHour

00 iMinute

0 iSecond

0.0 FracSecond

\#SAVERESTART

F DoSaveRestart

500 DnSaveRestart

-1. DtSaveRestart

\#TIMEACCURATE

F DoTimeAccurate

\#COUPLE2

GM NameSource

IE NameTarget

50 DnCouple

-1. DtCouple

! For implicit this improves performance

COUPLETIME

GM NameComp

F DoCoupleOnTime

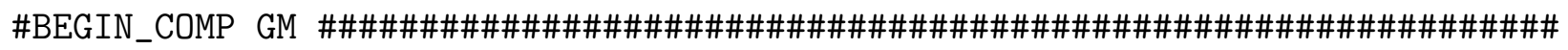

\#INCLUDE

. /SWMF.grid

\#OUTERBOUNDARY

outflow TypeBcEast

inflow TypeBcWest

float TypeBcSouth

float TypeBcNorth

float TypeBcBottom

float TypeBcTop

\#SOLARWIND

20.0

SwRhoDim [amu/cc]

232100.0

SwTDim [K]

$-400.0$

SwUxDim [km/s] 


$\begin{array}{rll}0.0 & \text { SwUyDim } & {[\mathrm{km} / \mathrm{s}]} \\ 0.0 & \text { SwUzDim } & {[\mathrm{km} / \mathrm{s}]} \\ 0.0 & \text { SwBxDim } & {[\mathrm{nT}]} \\ 0.0 & \text { SwByDim } & {[\mathrm{nT}]} \\ -20.0 & \text { SwBzDim } & {[\mathrm{nT}]}\end{array}$

\#TIMESTEPPING

1 nStage

0.8 CflExpl

\#SCHEME

1 nOrder

Rusanov TypeFlux

\#INNERBOUNDARY

ionosphere TypeBcInner

\#HALLRESISTIVITY

F UseHallResist

65. 0 HallFactorMax

0.5 HallCmaxFactor

\#HALLREGION

all NameHallRegion

\#SAVEPLOT

2 nPlotfile

$\mathrm{y}=0$ MHD idl StringPlot

2000 DnSavePlot

-1. DtSavePlot

-1. DxSavePlot

$z=0$ MHD idl StringPlot

2000 DnSavePlot

-1. DtSavePlot

-1. DxSavePlot

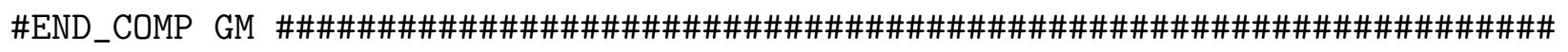

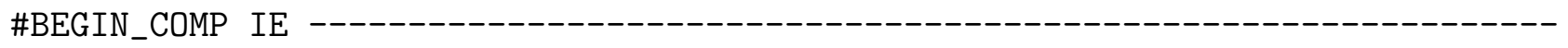

\#IONOSPHERE

1 conductance_model

F UseFullCurrent 
F UseFakeRegion2

150. F10.7 Flux

5 StarLightPedConductance

5 PolarCapPedConductance

\#SAVEPLOT

$1 \mathrm{nFile}$

max idl StringPlot

2000 DnOutput

-1. DtOutput

\#END_COMP IE

\#STOP

2000 MaxIteration

-1. tSimulationMax

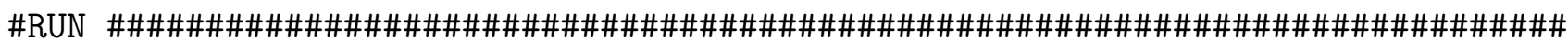

\#TIMEACCURATE

T DoTimeAccurate

\#COUPLE2

GM NameSource

IE NameTarget

-1 DnCouple

1. DtCouple

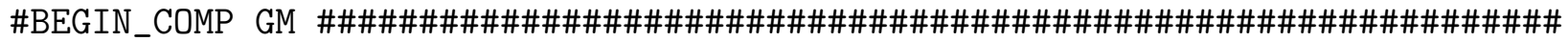

\#TIMESTEPPING

2 nStage

0.8 CflExpl

\#SCHEME

2 nOrder

Rusanov TypeFlux

minmod LimiterBeta

\#INNERBOUNDARY

ionosphere TypeBcInner

\#HALLRESISTIVITY 
F UseHallResist

65. 0 HallFactorMax

0.5 HallCmaxFactor

\#HALLREGION

all NameHallRegion

\#SAVEPLOT

2 nPlotfile

$y=0$ MHD idl StringPlot

-1 DnSavePlot

0.5 DtSavePlot

-1. DxSavePlot

$z=0$ MHD idl StringPlot

-1 DnSavePlot

0.5 DtSavePlot

-1. DxSavePlot

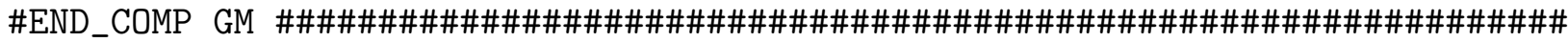

\#BEGIN_COMP IE

\#IONOSPHERE

1 conductance_model

F UseFullCurrent

F UseFakeRegion2

150. F10.7 Flux

5 StarLightPedConductance

5 PolarCapPedConductance

\#SAVEPLOT

1 nPlotfile

$\max$ idl StringPlot

-1 DnOutput

0.5 DtOutput

\#END_COMP IE

\#STOP

-1 MaxIteration

1.5 tSimulationMax

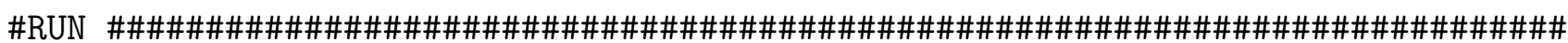


\#END 


\section{Appendix $\mathrm{F}$}

\section{SWMF Grid File}

The code below specifies the grid resolution used in the SWMF's Global Magnetosphere (GM) component. This is the grid CCMC_Komar_16M_CONFIRMED.grid referenced in the batch script provided in Appendix D.

! Sets up grid with CCMC's 2M grid cell resolution combined with the ! highest resolution domain of Colin Komar's CCMC runs.

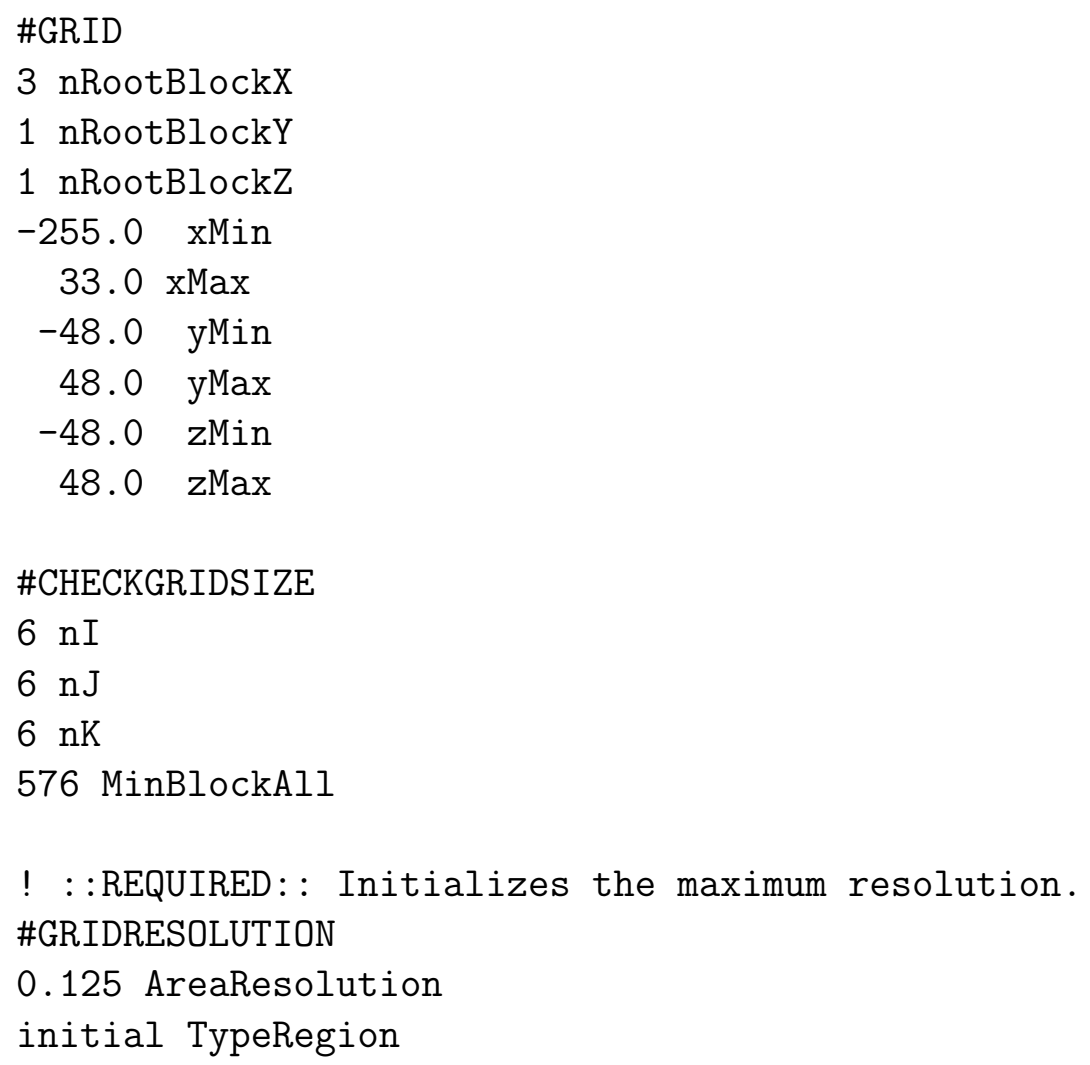


! : :REQUIRED:: Initializes the minimum resolution. \#GRIDRESOLUTION

8.0 AreaResolution

all TypeRegion

\#GRIDRESOLUTION

4.0 AreaResolution

box TypeRegion

-159.0 xMinBox

-128.0 yMinBox

-128.0 zMinBox

128.0 xMaxBox

128. 0 yMaxBox

$128.0 \mathrm{zMaxBox}$

\#GRIDRESOLUTION

2.0 AreaResolution

box TypeRegion

-39.0 xMinBox

-128.0 yMinBox

-128.0 zMinBox

128.0 xMaxBox

128. 0 yMaxBox

128.0 zMaxBox

\#GRIDRESOLUTION

2.0 AreaResolution

box TypeRegion

-135.0 xMinBox

-48.0 yMinBox

-24.0 zMinBox

48.0 xMaxBox

48.0 yMaxBox

24.0 zMaxBox

\#GRIDRESOLUTION

1.0 AreaResolution

box TypeRegion

-15.0 xMinBox

-24.0 yMinBox

-24.0 zMinBox

33.0 xMaxBox 
24.0 yMaxBox

24.0 zMaxBox

\#GRIDRESOLUTION

1.0 AreaResolution

box TypeRegion

-111.0 xMinBox

-24.0 yMinBox

-12.0 zMinBox

0.0 xMaxBox

24.0 yMaxBox

12.0 zMaxBox

\#GRIDRESOLUTION

0.5 AreaResolution

box TypeRegion

-9.0 xMinBox

-18.0 yMinBox

-18.0 zMinBox

15.0 xMaxBox

18.0 yMaxBox

18.0 zMaxBox

\#GRIDRESOLUTION

0.5 AreaResolution

box TypeRegion

-9.0 xMinBox

-12.0 yMinBox

-12.0 zMinBox

21.0 xMaxBox

12.0 yMaxBox

12.0 zMaxBox

\#GRIDRESOLUTION

0.5 AreaResolution

box TypeRegion

-99.0 xMinBox

-12.0 yMinBox

-6.0 zMinBox

15.0 xMaxBox

12.0 yMaxBox

6.0 zMaxBox 


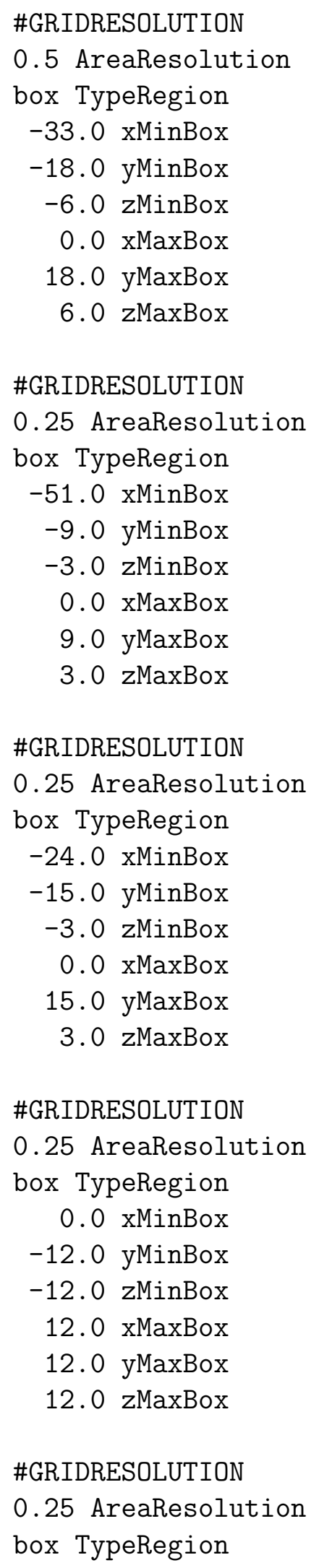




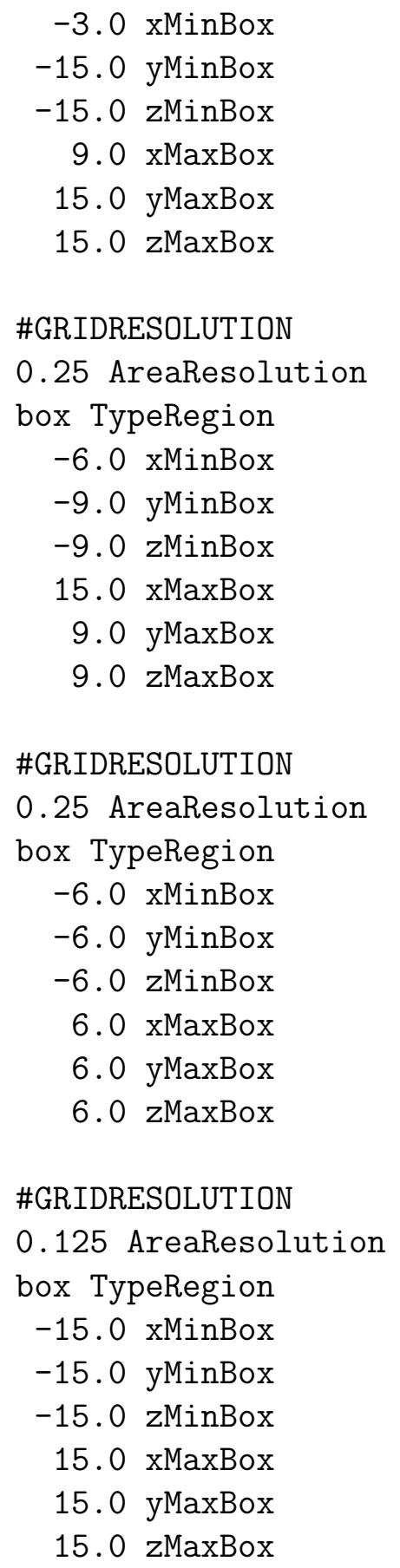




\section{Bibliography}

Alexeev, I. I., D. G. Sibeck, and S. Y. Bobrovnikov (1998), Concerning the location of magnetopause merging as a function of the magnetopause current strength, J. Geophys. Res., $103(\mathrm{~A} 4), 6675-6684$.

Alfvén, H. (1942), Existence of electromagnetic-hydrodynamic waves, Nature, 150, 405-406.

Anekallu, C. R., M. Palmroth, H. E. J. Koskinen, E. Lucek, and I. Dandouras (2013), Spatial variation of energy conversion at the Earth's magnetopause: Statistics from Cluster observations, J. Geophys. Res., 118(5), 1948-1959.

Baker, D. N., J. H. Allen, S. G. Kanekal, and G. D. Reeves (1998), Disturbed space environment may have been related to pager satellite failure, Eos Trans. AGU, 79(40), 477-483.

Baumjohann, W., and R. A. Treumann (2012), Basic Space Plasma Physics: Revised Edition, 496 pp., Imperial College Press.

Beidler, M. T., and P. A. Cassak (2011), Model for incomplete reconnection in sawtooth crashes, Phys. Rev. Lett., 10\%, 255002.

Birn, J., J. F. Drake, M. A. Shay, B. N. Rogers, R. E. Denton, M. Hesse, M. Kuznetsova, Z. W. Ma, A. Bhattacharjee, A. Otto, and P. L. Pritchett (2001), GEM magnetic reconnection challenge, J. Geophys. Res., 106, 3715. 
Biskamp, D. (1986), Magnetic reconnection via current sheets, Phys. Fluids, 29(5), 15201531.

Borovsky, J., B. Lotko, V. Uritsky, and J. Valdivia (2013a), Proposal for a GEM Focus Group: "Geospace Systems Science", selected as GEM Focus Group 2014-2018.

Borovsky, J. E. (2008), The rudiments of a theory of solar wind/magnetosphere coupling derived from first principles, J. Geophys. Res., 113, A08228.

Borovsky, J. E. (2013), Physical improvements to the solar wind reconnection control function for the Earth's magnetosphere, J. Geophys. Res., 118(5), 2113-2121.

Borovsky, J. E. (2014), Feedback of the magnetosphere, Science, 343(6175), 1086-1087.

Borovsky, J. E., and M. H. Denton (2006), Effect of plasmaspheric drainage plumes on solar-wind/magnetosphere coupling, Geophys. Res. Lett., $33(20)$, L20101.

Borovsky, J. E., and M. H. Denton (2009), Relativistic-electron dropouts and recovery: A superposed epoch study of the magnetosphere and the solar wind, J. Geophys. Res., $114(\mathrm{~A} 2), \mathrm{A} 02201$.

Borovsky, J. E., and J. T. Steinberg (2006), The "calm before the storm" in CIR/magnetosphere interactions: Occurrence statistics, solar wind statistics, and magnetospheric preconditioning, J. Geophys. Res., $111(\mathrm{~A} 7)$, A07S10.

Borovsky, J. E., M. Hesse, J. Birn, and M. M. Kuznetsova (2008), What determines the reconnection rate at the dayside magnetosphere?, J. Geophys. Res., 113, A07210.

Borovsky, J. E., M. H. Denton, R. E. Denton, V. K. Jordanova, and J. Krall (2013b), Estimating the effects of ionospheric plasma on solar wind/magnetosphere coupling via 
mass loading of dayside reconnection: Ion-plasma-sheet oxygen, plasmaspheric drainage plumes, and the plasma cloak, J. Geophys. Res., 118(9), 5695-5719.

Boudouridis, A., E. Zesta, L. R. Lyons, P. C. Anderson, and D. Lummerzheim (2005), Enhanced solar wind geoeffectiveness after a sudden increase in dynamic pressure during southward IMF orientation, J. Geophys. Res, $110(\mathrm{~A} 5)$, A05214.

Cassak, P. A. (2011), Theory and simulations of the scaling of magnetic reconnection with symmetric shear flow, Phys. Plasmas, 18(7), 072106.

Cassak, P. A., and A. Otto (2011), Scaling of the magnetic reconnection rate with symmetric shear flow, Phys. Plasmas, 18(7), 074501.

Cassak, P. A., and M. A. Shay (2007), Scaling of asymmetric magnetic reconnection: General theory and collisional simulations, Phys. Plasmas, 14, 102114.

Cassak, P. A., and M. A. Shay (2008), Scaling of asymmetric Hall reconnection, Geophys. Res. Lett., 35, L19102.

Cassak, P. A., and M. A. Shay (2009), Structure of the dissipation region in fluid simulations of asymmetric magnetic reconnection, Phys. Plasmas, 16, 055704.

Chen, L., R. M. Thorne, V. K. Jordanova, C.-P. Wang, M. Gkioulidou, L. Lyons, and R. B. Horne (2010), Global simulation of EMIC wave excitation during the 21 April 2001 storm from coupled RCM-RAM-HOTRAY modeling, J. Geophys. Res., $115(\mathrm{~A} 7)$, A07209.

Chen, X. L., and P. J. Morrison (1990), Resistive tearing instability with equilibrium shear flow, Phys. Fluids B, 2(3), 495-507. 
Cnossen, I., M. Wiltberger, and J. E. Ouellette (2012), The effects of seasonal and diurnal variations in the Earth's magnetic dipole orientation on solar wind-magnetosphereionosphere coupling, J. Geophys. Res., 117(A11), A11211.

Cooling, B. M. A., C. J. Owen, and S. J. Schwartz (2001), Role of the magnetosheath flow in determining the motion of open flux tubes, J. Geophys. Res., 106(A9), 18763-18775.

Cornwall, J. M. (1965), Cyclotron instabilities and electromagnetic emission in the ultra low frequency and very low frequency ranges, J. Geophys. Res., 70(1), 61-69.

Cowley, S. W. H. (1973), A qualitative study of the reconnection between the Earth's magnetic field and an interplanetary field of arbitrary orientation, Radio Sci., 8(11), 903-913.

Cowley, S. W. H. (1976), Comments on the merging of nonantiparallel magnetic fields, J. Geophys. Res., 81(19), 3455-3458.

Crooker, N. U. (1979), Dayside merging and cusp geometry, J. Geophys. Res., 84(A3), 951959.

De Zeeuw, D., T. Gombosi, C. Groth, K. Powell, and Q. Stout (2000), An adaptive MHD method for global space weather simulations, IEEE T. Plasma Sci., 28, 1956.

DiBraccio, G. A., J. A. Slavin, S. A. Boardsen, B. J. Anderson, H. Korth, T. H. Zurbuchen, J. M. Raines, D. N. Baker, R. L. McNutt, and S. C. Solomon (2013), MESSENGER observations of magnetopause structure and dynamics at Mercury, J. Geophys. Res., 118(3), $997-1008$.

Dorelli, J. C., and A. Bhattacharjee (2008), Defining and identifying three-dimensional 
magnetic reconnection in resistive magnetohydrodynamic simulations of Earth's magnetosphere, Phys. Plasmas, 15, 056504.

Dorelli, J. C., and A. Bhattacharjee (2009), On the generation and topology of flux transfer events, J. Geophys. Res., 114, A06213.

Dorelli, J. C., M. Hesse, M. M. Kuznetsova, L. Rastaetter, and J. Raeder (2004), A new look at driven magnetic reconnection at the terrestrial subsolar magnetopause, J. Geophys. Res., 109(A12), A12216.

Dorelli, J. C., A. Bhattacharjee, and J. Raeder (2007), Separator reconnection at Earth's dayside magnetopause under generic northward interplanetary magnetic field conditions, J. Geophys. Res., 112, A02202.

Dorelli, J. C., A. Glocer, G. Collinson, and G. Toth (2015), The role of the Hall effect in the global structure and dynamics of planetary magnetospheres: Ganymede as a case study, submitted to J. Geophys. Res. December 2014, arXiv:1501.00501.

Doss, C. E., C. M. Komar, P. A. Cassak, F. D. Wilder, S. Eriksson, and J. F. Drake (in prep.), Asymmetric reconnection with a shear flow and applications to X-line motion at the polar cusps, to be submitted to J. Geophys. Res.

Dungey, J. W. (1961), Interplanetary magnetic field and the auroral zones, Phys. Rev. Lett., 6,47 .

Dungey, J. W. (1963), The structure of the exosphere, or adventures in velocity space, in Geophysics: The Earth's Environment, edited by C. De Witt, J. Hieblot, and A. Lebeau, p. 505, Gordon Breach, New York. 
Dunlop, M. W., Q.-H. Zhang, Y. V. Bogdanova, K. J. Trattner, Z. Pu, H. Hasegawa, J. Berchem, M. G. G. T. Taylor, M. Volwerk, J. P. Eastwood, B. Lavraud, C. Shen, J.-K. Shi, J. Wang, D. Constantinescu, A. N. Fazakerley, H. Frey, D. Sibeck, P. Escoubet, J. A. Wild, Z. X. Liu, and C. Carr (2011), Magnetopause reconnection across wide local time, Ann. Geophys., 29(9), 1683-1697.

Fuselier, S. A., K. J. Trattner, and S. M. Petrinec (2011), Antiparallel and component reconnection at the dayside magnetopause, J. Geophys. Res., 116(A10), A10227.

Fuselier, S. A., R. Frahm, W. S. Lewis, A. Masters, J. Mukherjee, S. M. Petrinec, and I. J. Sillanpaa (2014), The location of magnetic reconnection at Saturn's magnetopause: A comparison with Earth, J. Geophys. Res., 119(4), 2563-2578.

Glocer, A., J. Dorelli, G. Tòth, C. Komar, and P. Cassak (2015), Techniques for finding magnetic separators in reconnecting systems, to be submitted to J. Geophys. Res.

Gombosi, T., D. DeZeeuw, C. Groth, and K. Powell (2000), Magnetospheric configuration for Parker-spiral IMF conditions: Results of a 3D AMR MHD simulation, Adv. Space Res., 26, 139 .

Gonzalez, W. D., and F. S. Mozer (1974), A quantitative model for the potential resulting from reconnection with an arbitrary interplanetary magnetic field, J. Geophys. Res., $79(28), 4186-4194$.

Gonzalez, W. D., B. T. Tsurutani, and A. L. Clúa de Gonzalez (1999), Interplanetary origin of geomagnetic storms, Space Sci. Rev., 88(3-4), 529-562.

Gosling, J. T. (2007), Observations of magnetic reconnection in the turbulent high-speed solar wind, Ap. J. Lett., 671(1), L73. 
Gosling, J. T., and T. D. Phan (2013), Magnetic reconnection in the solar wind at current sheets associated with extremely small field shear angles, Ap. J. Lett., 763(2), L39.

Gosling, J. T., T. D. Phan, R. P. Lin, and A. Szabo (2007), Prevalence of magnetic reconnection at small field shear angles in the solar wind, Geophys. Res. Lett., 34 (15), L15110.

Greene, J. M. (1988), Geometrical properties of three-dimensional reconnecting magnetic fields with nulls, J. Geophys. Res., 93, 8583.

Guo, R., Z. Pu, C. Xiao, X. Wang, S. Fu, L. Xie, Q. Zong, J. He, Z. Yao, J. Zhong, and J. Li (2013), Separator reconnection with antiparallel/component features observed in magnetotail plasmas, J. Geophys. Res., 118, 6116-6126.

Haynes, A. L., and C. E. Parnell (2007), A trilinear method for finding null points in a three-dimensional vector space, Phys. Plasmas, 14, 082107.

Haynes, A. L., and C. E. Parnell (2010), A method for finding three-dimensional magnetic skeletons, Phys. Plasmas, 17, 092903.

Haynes, A. L., C. E. Parnell, K. Galsgaard, and E. R. Priest (2007), Magnetohydrodynamic evolution of magnetic skeletons, Proc. R. Soc. A, vol. 463, 1097-1115.

Hesse, M., N. Aunai, S. Zenitani, M. Kuznetsova, and J. Birn (2013), Aspects of collisionless magnetic reconnection in asymmetric systems, Phys. Plasmas, 20 (6), 061210.

Hoilijoki, S., V. M. Souza, B. M. Walsh, P. Janhunen, and M. Palmroth (2014), Magnetopause reconnection and energy conversion as influenced by the dipole tilt and the IMF $B_{x}$, J. Geophys. Res., $119(6), 4484-4494$. 
Hoppe, H., T. DeRose, T. Duchamp, J. McDonald, and W. Stuetzle (1992), Surface reconstruction from unorganized points, SIGGRAPH Comput. Graph., 26(2), 71-78.

Hu, Y. Q., Z. Peng, C. Wang, and J. R. Kan (2009), Magnetic merging line and reconnection voltage versus IMF clock angle: Results from global MHD simulations, J. Geophys. Res., 114, A08220.

Jordanova, V. K., M. Spasojevic, and M. F. Thomsen (2007), Modeling the electromagnetic ion cyclotron wave-induced formation of detached subauroral proton arcs, J. Geophys. Res., 112(A8), A08209.

Kelley, M. C. (2009), The Earth's Ionosphere: Plasma Physics and Electrodynamics: Second Edition, Academic Press.

Kennel, C. F., and H. E. Petschek (1966), Limit on stably trapped particle fluxes, J. Geophys. Res., 71(1), 1-28.

Kivelson, M. G., and C. T. Russell (Eds.) (1995), Introduction to Space Physics, Cambridge University Press.

Komar, C. M., P. A. Cassak, J. C. Dorelli, A. Glocer, and M. M. Kuznetsova (2013), Tracing magnetic separators and their dependence on IMF clock angle in global magnetospheric simulations, J. Geophys. Res., 118(8), 4998-5007.

Komar, C. M., R. L. Fermo, and P. A. Cassak (2015), Comparative analysis of dayside magnetic reconnection models in global magnetosphere simulations, J. Geophys. Res., 120(1), 276-294. 
La Belle-Hamer, A. L., A. Otto, and L. C. Lee (1994), Magnetic reconnection in the presence of sheared plasma flow: Intermediate shock formation, Phys. Plasmas, 1(3), 706-713.

La Belle-Hamer, A. L., A. Otto, and L. C. Lee (1995), Magnetic reconnection in the presence of sheared flow and density asymmetry: Applications to the Earth's magnetopause, J. Geophys. Res., 100(A7), 11875-11889.

Laitinen, T. V., P. Janhunen, T. I. Pulkkinen, M. Palmroth, and H. E. J. Koskinen (2006), On the characterization of magnetic reconnection in global MHD simulations, Ann. Geophys., 24, 3059 .

Laitinen, T. V., M. Palmroth, T. I. Pulkkinen, P. Janhunen, and H. E. J. Koskinen (2007), Continuous reconnection line and pressure-dependent energy conversion on the magnetopause in a global MHD model, J. Geophys. Res., 112, A11201.

Lau, Y.-T., and J. M. Finn (1990), Three-dimensional kinematic reconnection in the presence of field nulls and closed field lines, Astrophys. J., 350, 672.

Lavraud, B., and J. E. Borovsky (2008), Altered solar wind-magnetosphere interaction at low Mach numbers: Coronal mass ejections, J. Geophys. Res., 113(A9), A00B08.

Lavraud, B., M. F. Thomsen, J. E. Borovsky, M. H. Denton, and T. I. Pulkkinen (2006), Magnetosphere preconditioning under northward IMF: Evidence from the study of coronal mass ejection and corotating interaction region geoeffectiveness, J. Geophys. Res., $111(\mathrm{~A} 9), \mathrm{A} 09208$.

Lavraud, B., E. Larroque, E. Budnik, V. Génot, J. E. Borovsky, M. W. Dunlop, C. Foullon, H. Hasegawa, C. Jacquey, K. Nykyri, A. Ruffenach, M. G. G. T. Taylor, I. Dandouras, 
and H. Rème (2013), Asymmetry of magnetosheath flows and magnetopause shape during low Alfvén Mach number solar wind, J. Geophys. Res., 118(3), 1089-1100.

Lindeberg, T. (1993), Discrete derivative approximations with scale-space properties: A basis for low-level feature extraction, J. Math. Imaging Vision, 3(4), 349-376.

Lindeberg, T. (1998), Edge detection and ridge detection with automatic scale selection, Int. J. Comput. Vision, 30(2), 117-156.

Liu, Z.-Q., J. Y. Lu, K. Kabin, Y. F. Yang, M. X. Zhao, and X. Cao (2012), Dipole tilt control of the magnetopause for southward IMF from global magnetohydrodynamic simulations, J. Geophys. Res., 117(A7), A09237.

Lopez, R. E., R. Bruntz, E. J. Mitchell, M. Wiltberger, J. G. Lyon, and V. G. Merkin (2010), Role of magnetosheath force balance in regulating the dayside reconnection potential, J. Geophys. Res., 115(A12), A12216.

Lu, J. Y., Z.-Q. Liu, K. Kabin, M. X. Zhao, D. D. Liu, Q. Zhou, and Y. Xiao (2011), Three dimensional shape of the magnetopause: Global MHD results, J. Geophys. Res., 116(A9), A07207.

Malakit, K., M. A. Shay, P. A. Cassak, and C. Bard (2010), Scaling of asymmetric magnetic reconnection: Kinetic particle-in-cell simulations, J. Geophys. Res., 115, A10223.

Moore, T. E., M.-C. Fok, and M. O. Chandler (2002), The dayside reconnection X line, J. Geophys. Res., 107(A10), 1332.

Moore, T. E., M.-C. Fok, D. C. Delcourt, S. P. Slinker, and J. A. Fedder (2008), Plasma plume circulation and impact in an MHD substorm, J. Geophys. Res., 113(A6), A06219. 
Mozer, F. S., and A. Hull (2010), Scaling the energy conversion rate from magnetic field reconnection to different bodies, Phys. Plasmas, $17(10), 102906$.

Murphy, N. A., C. R. Sovinec, and P. A. Cassak (2010), Magnetic reconnection with asymmetry in the outflow direction, J. Geophys. Res., $115(\mathrm{~A} 9)$, A09206.

National Research Council (2002), Storms from the Sun: The Emerging Science of Space Weather, The National Academies Press, Washington, DC.

National Research Council (2008), Severe Space Weather Events-Understanding Societal and Economic Impacts: A Workshop Report, The National Academies Press, Washington, DC.

Newell, P. T., T. Sotirelis, K. Liou, C.-I. Meng, and F. J. Rich (2007), A nearly universal solar wind-magnetosphere coupling function inferred from 10 magnetospheric state variables, J. Geophys. Res., 112(A1), A01206.

Němeček, Z., J. Šafránkova, A. Koval, J. Merka, and L. Přech (2011), MHD analysis of propagation of an interplanetary shock across magnetospheric boundaries, J. Atmos. Sol. Terr. Phys., 73(1), 20-29.

Olson, D. K., J. Dorelli, W. S. Daughton, and H. Karimabadi (2013), Locating magnetic reconnection $\mathrm{x}$-lines in 3D PIC simulations, abstract SM13A-2122 presented at 2013 Fall Meeting, AGU, San Francisco, Calif., 9-13 Dec.

Ouellette, J. E., B. N. Rogers, M. Wiltberger, and J. G. Lyon (2010), Magnetic reconnection at the dayside magnetopause in global Lyon-Fedder-Mobarry simulations, J. Geophys. Res., 115, A08222. 
Ouellette, J. E., J. G. Lyon, and B. N. Rogers (2014), A study of asymmetric reconnection scaling in the Lyon-Fedder-Mobarry code, J. Geophys. Res., 119(3), 1673-1682.

Palmroth, M., T. I. Pulkkinen, P. Janhunen, and C.-C. Wu (2003), Stormtime energy transfer in global MHD simulation, J. Geophys. Res., 108(A1), 1048.

Palmroth, M., R. C. Fear, and I. Honkonen (2012), Magnetopause energy transfer dependence on the interplanetary magnetic field and the Earth's magnetic dipole axis orientation, Ann. Geophys., 30(3), 515-526.

Papadopoulos, K., C. Goodrich, M. Wiltberger, R. Lopez, and J. Lyon (1999), The physics of substorms as revealed by the ISTP, Phys. Chem. Earth Part C, 24(1-3), 189-202.

Park, K. S., T. Ogino, and R. J. Walker (2006), On the importance of antiparallel reconnection when the dipole tilt and IMF $B_{y}$ are nonzero, J. Geophys. Res., 111 (A5), A05202.

Parker, E. N. (1957), Sweet's mechanism for merging magnetic fields in conducting fluids, J. Geophys. Res., 62, 509.

Parker, E. N. (1958), Dynamics of the interplanetary gas and magnetic fields, Astrophys. J., 128,664 .

Parnell, C. E., J. M. Smith, T. Neukirch, and E. R. Priest (1996), The structure of threedimensional magnetic neutral points, Phys. Plasmas, 3, 759.

Parnell, C. E., A. L. Haynes, and K. Galsgaard (2010), Structure of magnetic separators and separator reconnection, J. Geophys. Res., 115(A2), A02102.

Paschmann, G. (2008), Recent in-situ observations of magnetic reconnection in near-Earth space, Geophys. Res. Lett., 35(19), L19109. 
Peng, Z., C. Wang, and Y. Q. Hu (2010), Role of IMF $B_{x}$ in the solar wind-magnetosphereionosphere coupling, J. Geophys. Res., 115, A08224.

Phan, T. D., J. T. Gosling, and M. S. Davis (2009), Prevalence of extended reconnection X-lines in the solar wind at 1 AU, Geophys. Res. Lett., 36(9), L09108.

Phan, T. D., J. T. Gosling, G. Paschmann, C. Pasma, J. F. Drake, M. Øieroset, D. Larson, R. P. Lin, and M. S. Davis (2010), The dependence of magnetic reconnection on plasma $\beta$ and magnetic shear: Evidence from solar wind observations, Ap. J. Lett., $719(2)$, L199.

Pickett, J. S., B. Grison, Y. Omura, M. J. Engebretson, I. Dandouras, A. Masson, M. L. Adrian, O. Santolík, P. M. E. Décréau, N. Cornilleau-Wehrlin, and D. Constantinescu (2010), Cluster observations of EMIC triggered emissions in association with Pc1 waves near Earth's plasmapause, Geophys. Res. Lett., 37(9), L09104.

Powell, K. G., P. L. Roe, T. J. Linde, T. I. Gombosi, and D. L. D. Zeeuw (1999), A solutionadaptive upwind scheme for ideal magnetohydrodynamics, J. Comp. Phys., 154, 284.

Pu, Z. Y., J. Raeder, J. Zhong, Y. V. Bogdanova, M. Dunlop, C. J. Xiao, X. G. Wang, and A. Fazakerley (2013), Magnetic topologies of an in vivo FTE observed by Double Star/TC-1 at Earth's magnetopause, Geophys. Res. Lett., 40(14), 3502-3506.

Pulkkinen, T. I., M. Palmroth, H. E. J. Koskinen, T. V. Laitinen, C. C. Goodrich, V. G. Merkin, and J. G. Lyon (2010), Magnetospheric modes and solar wind energy coupling efficiency, J. Geophys. Res., 115 (A3), A03207.

Raeder, J. (1999), Modeling the magnetosphere for northward interplanetary magnetic field: Effects of electrical resistivity, J. Geophys. Res., 104, 17357. 
Rosenberg, M. J., C. K. Li, W. Fox, I. Igumenshchev, F. H. Séguin, R. P. J. Town, J. A. Frenje, C. Stoeckl, V. Glebov, and R. D. Petrasso (2015), A laboratory study of asymmetric magnetic reconnection in strongly driven plasmas, Nat. Comm., 6 .

Russell, C. T., and R. C. Elphic (1978), Initial ISEE magnetometer results: magnetopause observations, Space Sci. Rev., 22, 681.

Schindler, K., M. Hesse, and J. Birn (1988), General magnetic reconnection, parallel electric fields, and helicity, J. Geophys. Res., 93, 5547.

Schreier, R., M. Swisdak, J. F. Drake, and P. A. Cassak (2010), Three-dimensional simulations of the orientation and structure of reconnection X-lines, Phys. Plasmas, 17(11), 110704.

Shabansky, V. P. (1971), Some processes in the magnetosphere, Space Sci. Rev., 12(3), 299-418.

Siscoe, G. L. (1987), The magnetospheric boundary, in Physics of Space Plasmas, edited by T. Chang, G. B. Crew, and J. R. Jasperse, pp. 3-78, Scientific, Cambridge, Mass.

Siscoe, G. L., G. M. Erickson, B. U. Ö. Sonnerup, N. C. Maynard, K. D. Siebert, D. R. Weimer, and W. W. White (2001), Global role of $E_{\|}$in magnetopause reconnection: An explicit demonstration, J. Geophys. Res., 106, 13015.

Siscoe, G. L., G. M. Erickson, B. U. Ö. Sonnerup, N. C. Maynard, J. A. Schoendorf, K. D. Siebert, D. R. Weimer, W. W. White, and G. R. Wilson (2002), Flow-through magnetic reconnection, Geophys. Res. Lett., 29, 1626. 
Sonnerup, B. U. Ö. (1974), Magnetopause reconnection rate, J. Geophys. Res., 79(10), $1546-1549$.

Sonnerup, B. U. Ö., and L. J. Cahill (1967), Magnetopause structure and attitude from Explorer 12 observations, J. Geophys. Res., 72(1), 171-183.

Sweet, P. A. (1958), The neutral point theory of solar flares, in Electromagnetic Phenomena in Cosmical Physics, IAU Symposium, vol. 6, edited by B. Lehnert, p. 123.

Swisdak, M., and J. F. Drake (2007), Orientation of the reconnection X-line, Geophys. Res. Lett., $34(11)$, L11106.

Swisdak, M., J. F. Drake, M. A. Shay, and B. N. Rogers (2003), Diamagnetic suppression of component magnetic reconnection at the magnetopause, J. Geophys. Res., 108(A5), 1218.

Swisdak, M., M. Opher, J. F. Drake, and F. A. Bibi (2010), The vector direction of the interstellar magnetic field outside the heliosphere, Astrophys. J., $710(2), 1769$.

Teh, W.-L., and B. U. Ö. Sonnerup (2008), First results from ideal 2-D MHD reconstruction: magnetopause reconnection event seen by Cluster, Ann. Geophys., 26(9), 2673-2684.

Thomsen, M. F., J. E. Borovsky, R. M. Skoug, and C. W. Smith (2003), Delivery of cold, dense plasma sheet material into the near-Earth region, J. Geophys. Res., 108(A4), 1151.

Thorne, R. M. (2010), Radiation belt dynamics: The importance of wave-particle interactions, Geophys. Res. Lett., 37(22), L22107.

Tóth, G., I. V. Sokolov, T. I. Gombosi, D. R. Chesney, C. R. Clauer, D. L. De Zeeuw, K. C. Hansen, K. J. Kane, W. B. Manchester, R. C. Oehmke, K. G. Powell, A. J. Ridley, I. I. Roussev, Q. F. Stout, O. Volberg, R. A. Wolf, S. Sazykin, A. Chan, B. Yu, and J. Kóta 
(2005), Space Weather Modeling Framework: A new tool for the space science community, J. Geophys. Res., $110(\mathrm{~A} 12), \mathrm{A} 12226$.

Tóth, G., B. van der Holst, I. V. Sokolov, D. L. D. Zeeuw, T. I. Gombosi, F. Fang, W. B. Manchester, X. Meng, D. Najib, K. G. Powell, Q. F. Stout, A. Glocer, Y.-J. Ma, and M. Opher (2012), Adaptive numerical algorithms in space weather modeling, J. Comp. Phys., 231(3), 870-903.

Trattner, K. J., J. S. Mulcock, S. M. Petrinec, and S. A. Fuselier (2007), Probing the boundary between antiparallel and component reconnection during southward interplanetary magnetic field conditions, J. Geophys. Res., 112(A8), A08210.

Trattner, K. J., S. M. Petrinec, S. A. Fuselier, and T. D. Phan (2012), The location of reconnection at the magnetopause: Testing the maximum magnetic shear model with THEMIS observations, J. Geophys. Res., $117(\mathrm{~A} 1)$, A01201.

Tsyganenko, N. A. (1995), Modeling the Earth's magnetospheric magnetic field confined within a realistic magnetopause, J. Geophys. Res., 100(A4), 5599-5612.

Turner, D. L., Y. Shprits, M. Hartinger, and V. Angelopoulos (2012), Explaining sudden losses of outer radiation belt electrons during geomagnetic storms, Nat. Phys., 8(3), 208212.

Walsh, B. M., J. C. Foster, P. J. Erickson, and D. G. Sibeck (2014), Simultaneous groundand space-based observations of the plasmaspheric plume and reconnection, Science, $343(6175), 1122-1125$.

Wilder, F. D., S. Eriksson, K. J. Trattner, P. A. Cassak, S. A. Fuselier, and B. Lybekk (2014), Observation of a retreating $x$ line and magnetic islands poleward of the cusp 
during northward interplanetary magnetic field conditions, J. Geophys. Res., 119(12), $9643-9657$.

Xiao, C. J., X. G. Wang, Z. Y. Pu, H. Zhao, J. X. Wang, Z. W. Ma, S. Y. Fu, M. G. Kivelson, Z. X. Liu, Q. G. Zong, K. H. Glassmeier, A. Balogh, A. Korth, H. Reme, and C. P. Escoubet (2006), In situ evidence for the structure of the magnetic null in a 3D reconnection event in the Earth's magnetotail, Nat. Phys., 2, 478.

Xiao, C. J., X. G. Wang, Z. Y. Pu, Z. W. Ma, H. Zhao, G. P. Zhou, J. X. Wang, M. G. Kivelson, S. Y. Fu, Z. X. Liu, Q. G. Zong, M. W. Dunlop, K. H. Glassmeier, E. Lucek, H. Reme, I. Dandouras, and C. P. Escoubet (2007), Satellite observations of separator-line geometry of three-dimensional magnetic reconnection, Nat. Phys., 3, 609.

Yamada, M., H. Ji, S. Hsu, T. Carter, R. Kulsrud, N. Bretz, F. Jobes, Y. Ono, and F. Perkins (1997), Study of driven magnetic reconnection in a laboratory plasma, Phys. Plasmas, $4(5), 1936-1944$.

Yeh, T. (1976), Day side reconnection between a dipolar geomagnetic field and a uniform interplanetary field, J. Geophys. Res., 81, 2140.

Yoo, J., M. Yamada, H. Ji, J. Jara-Almonte, C. E. Myers, and L.-J. Chen (2014), Laboratory study of magnetic reconnection with a density asymmetry across the current sheet, Phys. Rev. Lett., 113, 095002. 